\title{
COHERENT MODES FOR MULTIPLE NON-RIGID BUNCHES IN A STORAGE RING
}

\author{
J. Scott Berg
}

SLAC-Report-478

March 1996

Prepared for the Department of Energy

under contract number DE-AC03-76SF00515 

This document and the material and data contained therein, was developed under sponsorship of the United States Government. Neither the United States nor the Department of Energy, nor the Leland Stanford Junior University, nor their employees, nor their respective contractors, subcontractors, or their employees, makes any warranty, express or implied, or assumes any liability or responsibility for accuracy, completeness or usefulness of any information, apparatus, product or process disclosed, or represents that its use will not infringe privately-owned rights. Mention of any product, its manufacturer, or suppliers shall not, nor is it intended to, imply approval, disapproval, or fitness for any particular use. A royalty-free, nonexclusive right to use and disseminate same for any purpose whatsoever, is expressly reserved to the United States and the Universitv. 
SLAC- -478

$\mathrm{UC}-414$

\section{COHERENT MODES FOR MULTIPLE NON-RIGID BUNCHES IN A STORAGE RING*}

\section{J. Scott Berg}

Stanford Linear Accelerator Center Stanford University, Stanford, CA 94309

\section{SLAC-REPORT-478}

March 1996

Prepared for the Department of Energy

under contract number DE-AC03-76SF00515

Printed in the United States of America. Available from the National Technical Information Service, U.S. Department of Commerce, 5285 Port Royal, Springfield, Virginia 22161.

\footnotetext{
Ph.D. thesis, Stanford University, Stanford, CA.
}

\section{Abstract}

A method is presented for determining the stability of a system consisting of several highly relativistic bunches of charged particles circulating in a storage ring. The particles interact with magnets designed to guide the beam as well as with electromagnetic fields induced by the particles themselves. Previous work has considered modes where all bunches in the ring are executing the same type of internal oscillation. This dissertation considers the results of allowing those modes to couple to one another. The formalism begins with a selfconsistent distribution, and analyzes small perturbations to that distribution to determine if they grow exponentially. The formalism allows one to do this computation for an arbitrary magnetic lattice, as well as an arbitrary distribution of wakefield sources around the ring. The method also allows for the inclusion of a feedback system which is designed to damp multibunch oscillations. The PEP-II $B$-factory with a linear lattice is used as an example to demonstrate and explain the phenomenology that results from this coupling of multibunch modes. The effect of adding feedback is also explored. 


\section{Acknowledgments}

I would like to thank all the people who have helped in this work, especially the following people.

First of all, Ron Ruth, who first realized that the problem of multibunch mode coupling could be an issue in the design of machines such as the PEP-II $B$ factory, and who has helped me throughout my work. Also, his excellent management of the Accelerator Theory and.Special Projects group at SLAC has created an environment where I had the tools and support I needed to complete my work.

I have also enjoyed working with Robert Warnock, with whom I had the opportunity to learn a lot about nonlinear dynamics. He started me off in accelerator physics by giving me the opportunity to work with him on some projects he had conceived, and the knowledge, experience, and notoriety I have received as a result have served me well.

Innumerable people in the Accelerator Theory and Special Projects group at SLAC, as well as those in other groups have provided useful help at various times, and I owe many thanks to them all. Yunhai Cai deserves special mention, since he furnished much of the lattice data I needed.

I would like to thank Roger Romani and Per Enge for agreeing to be on the reading and defense committee of some random graduate student who came knocking on their doors. I am also very grateful to Perry Wilson who not only agreed to be on my reading and defense committees, but also was a big help in finding other members. Alex Chao was also very helpful in agreeing to be on my defense committee at the last minute.

A special thanks goes to Teri Einloth, who helped keep me from starving to death in my time here.

Finally, I would like to thank the United States government, which has supported my work here through the Department of Energy, contract DE-AC03-76SF00515.

\section{Contents}

Abstract

ii

Acknowledgments

1 Introduction

2 Description of the Physical System

2.1 The Storage Ring $\ldots \ldots \ldots \ldots \ldots \ldots \ldots$

2.1 .1 Coordinate System . . . . . . . . . . . . . . 3

2.1 .2 Forces on a Particle . . . . . . . . . . . . 4

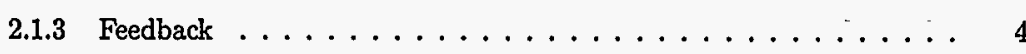

2.2 Oscillation Modes of the Beam $\ldots \ldots \ldots \ldots \ldots \ldots$

2.2 .1 Oscillations without Wakefields $\ldots \ldots \ldots \ldots \ldots \ldots \ldots$

2.2.2 Single Bunch Mode Coupling . . . . . . . . . . 7

2.2 .3 Multibunch Mode Coupling . . . . . . . . . . . . . . . . 8

3 Mathematical Description of the System 10

3.1 Wake Functions . . . . . . . . . . . . . . . . . . 10

3.1 .1 Force on a Test Charge $\ldots \ldots \ldots \ldots \ldots 11$

3.1 .2 Fields of a Source Distribution . . . . . . . . . . . 13

3.1.3 Wake Response to the Source . . . . . . . . . . . . . . 15

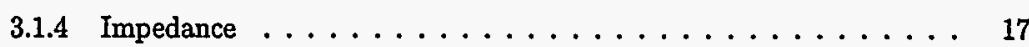

3.2 Hamiltonian for a Single Particle . . . . . . . . . . . . . . 17

3.2.1 General Electromagnetic Hamiltonian for a Storage

Ring . . . . . . . . . . . . . . . . 17

3.2.2 Expansion of the Magnetic Field . . . . . . . . . . . . . . 19 
3.2.3 R.F. Cavity Voltage . . . . . . . . . . . . . . . 21

3.2.4 Hamiltonian for Small Displacements . . . . . . . . . . . . 22

3.2.5 General Nonlinear Hamiltonian with Wakefields . . . . . . . . . 24

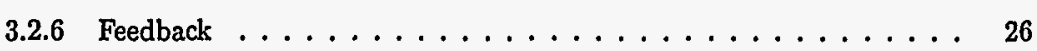

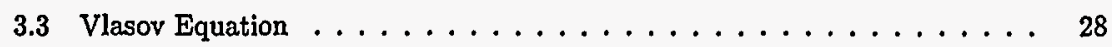

4 Eigenvalue System 31

4.1 Inversion of $G \ldots \ldots \ldots \ldots \ldots \ldots \ldots \ldots \ldots \ldots \ldots$

4.1.1 Fourier Analyze in All Components . . . . . . . . . . . . . 34

4.1.2 Fourier Analyze in All but One Component . . . . . . . . . 35

4.2 No Potential-Well Distortion $\ldots \ldots \ldots \ldots \ldots \ldots$

4.3 Linear Lattice without Dispersion . . . . . . . . . . . . . . . . . . . . . . . . . . . . . . . . . . . .

4.3.1 Computation of $R, B$, and $C \ldots \ldots \ldots \ldots \ldots \ldots \ldots$

4.3 .2 Gaussian Bunches . . . . . . . . . . . . . . . . . 42

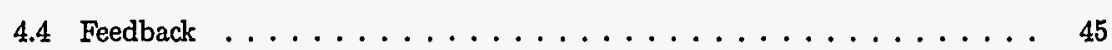

4.4 .1 No Potential-Well Distortion . . . . . . . . . . . . 47

4.4.2 Linear Lattice, No Dispersion, Gaussian Bunches . . . . . . . . . 47

5 Example and Analysis

5.1 Approximations Made . . . . . . . . . . . . . . . . . . . 49

5.2 Impedance Model . . . . . . . . . . . . . . . . 53

5.2 .1 Resistive Wall . . . . . . . . . . . . . . . . . 54

5.2 .2 Inductive $\ldots \ldots \ldots \ldots \ldots \ldots \ldots \ldots \ldots \ldots \ldots \ldots \ldots$

5.2 .3 Cavity Tails . . . . . . . . . . . . . . . . . 55

5.2 .4 Cavity Higher Order Modes . . . . . . . . . . . . . 55

5.2 .5 BPM Resonance . . . . . . . . . . . . . . . . . . 56

5.2 .6 Total Impedance $\ldots \ldots \ldots \ldots \ldots \ldots \ldots \ldots \ldots$

5.3 Frequencies and Growth Rates $\ldots \ldots \ldots \ldots \ldots \ldots$

5.3 .1 Single Bunch . . . . . . . . . . . . . . 57

5.3 .2 Multibunch Mode Frequencies . . . . . . . . . . . 62

5.3.3 $m=0$ Growth Rates . . . . . . . . . . . . . 65

$5.3 .4 \quad m=1$ Growth Rates . . . . . . . . . . . . . 67

5.3.5 $m=2$ Growth Rates . . . . . . . . . . . . . 69

5.3.6 $m=0$ Radial Mode Growth Rates . . . . . . . . . . 71
5.3.7 $m=3$ Growth Rates . . . . . . . . . . . . . . . 73

$5.3 .8 m=1$ Radial Mode Growth Rates . . . . . . . . . . . 74

5.3.9 Growth Rates for Fixed Current . . . . . . . . . . . . 76

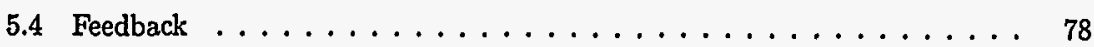

5.4 .1 Resistive Feedback . . . . . . . . . . . . . 80

5.4 .2 Reactive Feedback . . . . . . . . . . . . . 86

5.5 Landau Damping . . . . . . . . . . . . . . . . . . 91

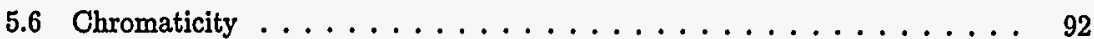

6 Conclusion 105

6.1 Uniqueness of this Approach $\ldots \ldots \ldots \ldots \ldots \ldots$

6.2 Summary of Results $\ldots \ldots \ldots \ldots \ldots \ldots \ldots$

$\begin{array}{ll}\text { A Symplectic Transformations } & 108\end{array}$

A.1 Shifts by Pure Time-Dependent Functions . . . . . . . . . . . . . . . . 108

A.2 Linear Time-Dependent Transformations . . . . . . . . . . . . . . . . . 109

A.3 Action-Angle Variables for One-Dimensional Linear

Time-Dependent Hamiltonian . . . . . . . . . . . . . . . . . . . . 109

A.4 Map for a One-Dimensional Single-Kick

Hamiltonian . . . . . . . . . . . . . . . . . . . . . 111

A.5 Nonlinear Normalization . . . . . . . . . . . . . . . . . . . 113

A.5.1 Action-Angle Lowest Order Transformation . . . . . . . . . . . 113

A.6 Synchrotron Tune Shift with Amplitude . . . . . . . . . . . . . . 114

$\begin{array}{ll}\text { B Action-Dependent Distribution Function } & 118\end{array}$

C Index of Symbols $\quad 120$ 


\section{List of Tables}

3.1 Lowest order values for $a_{n} . \ldots \ldots \ldots \ldots \ldots \ldots$

5.1 Parameters for the PEP-II low energy ring . . . . . . . . . . . . . 50

5.2 Vertical cavity higher order modes for PEP-II high power test cavity . . . .

\section{List of Figures}

2.1 Transverse $m=0$ (rigid) oscillation . . . . . . . . . . . 6

2.2 Transverse $m=1$ (head-tail) oscillation . . . . . . . . . . 6

2.3 Longitudinal $m=1$ (rigid) oscillation $\ldots \ldots \ldots \ldots \ldots \ldots \ldots$

2.4 Longitudinal $m=2$ oscillation $\ldots \ldots \ldots \ldots \ldots \ldots \ldots$

$5.1 \Delta \psi_{\tau}(s)$, plotted versus position in the ring . . . . . . . . . 53

5.2 Total impedance, multiplied by $\beta$-functions $\ldots \ldots \ldots \ldots$. . . . . . 57

5.3 Low frequency part of the total impedance. . . . . . . . . . 58

5.4 Form factors from equation $(5.8) \ldots \ldots \ldots \ldots \ldots \ldots$

5.5 Form factors of figure 5.4 over frequency range matching figure $5.3 . \ldots \ldots 60$

5.6 Single-bunch mode frequencies $\ldots \ldots \ldots \ldots \ldots$

5.7 Legend describing line types used in mode plots. . . . . . . . . . . . 62

5.8 Single-bunch growth rates . . . . . . . . . . . . . . . 63

5.9 Single-bunch frequencies, larger current range . . . . . . . . . . 64

5.10 Multibunch mode frequencies, without coupling . . . . . . . . . . . 65

5.11 Multibunch mode frequencies, with coupling . . . . . . . . . 66

$5.12 m=0$ mode growth rates, without coupling $\ldots \ldots \ldots \ldots 67 \ldots \ldots$

$5.13 m=0$ mode growth rates, with coupling between multibunch modes. . . . 68

$5.14 m=1$ multibunch mode growth rates, without coupling. . . . . . . . . 69

$5.15 m=1$ multibunch mode growth rates, with coupling. . . . . . . 70

$5.16 m=1$ multibunch mode growth rates, with coupling. Vertical scale is blown up from figure $5.15 \ldots \ldots \ldots \ldots \ldots \ldots \ldots$

$5.17 m=1$ multibunch mode growth rates, with coupling. Vertical scale is blown up from figure $5.16 \ldots \ldots \ldots \ldots \ldots \ldots \ldots \ldots$

$5.18 m=2$ multibunch mode growth rates, without coupling. . . . . . . . 73 
$5.19 m=2$ multibunch mode growth rates, with coupling. . . . . . . . . .

$5.20 m=2$ multibunch mode growth rates, with coupling. Vertical scale blown up from figure $5.19 . \ldots \ldots \ldots \ldots \ldots \ldots \ldots \ldots$

$5.21 m=0$ radial multibunch mode growth rates, without coupling. . . . . . . 76

$5.22 m=0$ radial multibunch mode growth rates, with coupling. . . . . . . 77

$5.23 m=3$ multibunch mode growth rates, without coupling. . . . . . . . . 78

$5.24 m=3$ multibunch mode growth rates, with coupling. . . . . . . . . . 79

$5.25 m=1$ radial multibunch mode growth rates, without coupling. . . . . . . 80

$5.26 m=1$ radial multibunch mode growth rates, with coupling . . . . . . 81

$5.27 m=1$ radial multibunch mode growth rates, with coupling. Vertical scale

blown up from figure $5.26 \ldots \ldots \ldots \ldots \ldots \ldots$. . . . . . . . . 82

5.28 Multibunch mode growth rates, without coupling, at 3.159 A. . . . . . . 83

5.29 Multibunch mode growth rates, with coupling, at 3.159 A. . . . . . . . . 84

5.30 Scaled feedback impedance from equation $(5.22) \ldots \ldots \ldots \ldots$

5.31 Multibunch mode frequencies, with feedback. . . . . . . . . . . 86

$5.32 m=0$ multibunch growth rates, with feedback. . . . . . . . . . . 87

$5.33 m=1$ multibunch growth rates, with feedback. . . . . . . . . 88

$5.34 m=1$ multibunch growth rates, with feedback. Vertical scale blown up from

figure $5.33 . \ldots \ldots \ldots \ldots \ldots \ldots$. . . . . . . . . . . . . 89

$5.35 m=1$ multibunch growth rates, with feedback but no coupling. . . . . . 90

$5.36 m=0$ radial multibunch growth rates, with feedback. . . . . . . . . 91

5.37 Multibunch mode growth rates at $3.159 \mathrm{~A}$, with feedback. . . . . . . . . 92

5.38 Multibunch mode frequencies with both resistive and reactive feedback. . . 93

$5.39 m=0$ radial mode mode growth/damping rates with resistive and reactive

feedback . . . . . . . . . . . . . . . . . . 94

$5.40 m=1$ multibunch mode growth/damping rates with resistive and reactive

feedback. . . . . . . . . . . . . . . . 95

$5.41 m=1$ radial multibunch mode growth/damping rates with resistive and

reactive feedback. . . . . . . . . . . . . . . . . . 96

5.42 Multibunch growth rates at $3.159 \mathrm{~A}$, with resistive and reactive feedback. . 97

5.43 Absolute value of multibunch mode shifts at 3.159 A . . . . . . . . . 98

5.44 Mode frequencies for a single bunch with $\xi=2 \ldots \ldots \ldots$. . . . . . 99

5.45 Growth rates for a single bunch with $\xi=2 \ldots \ldots \ldots \ldots$. . . . . 100
5.46 Growth rates for a single bunch with $\xi=2$. Scale blown up from figure 5.45. 101 $5.47 m=0$ mode growth rates, with $\xi=2 . \ldots \ldots \ldots \ldots 2 \ldots \ldots$ $5.48 m=1$ mode growth rates, with $\xi=2 \ldots \ldots \ldots \ldots$. . . . . . . 103 5.49 Multibunch growth rates at $3.159 \mathrm{~A}$, with $\xi=2 . \ldots \ldots$. . . . . . 104

B.1 Contours of integration for equation (B.7). 


\section{Chapter 1}

\section{Introduction}

The field of accelerator physics address the problem of creating and maintaining a beam of particles. This beam of particles is to be optimized for some purpose, such as the creation of certain events for a high-energy physics experiment, or the generation of radiation.

A storage ring is a device which keeps particles circulating near a long, closed curve (the ideal orbit). The question arises of whether particles which begin near that closed curve will remain near to it. This can be considered the central problem in accelerator beam dynamics.

There are two ways in which the stability of a particle beam is addressed. One is generally referred to as nonlinear dynamics. This asks whether (or over what range of initial conditions) a single particle will remain near the ideal orbit, when all other particles are ignored. The second way of addressing beam stability is to ask what effect all the particles in the beam have on each other-these are known as "collective effects."

The purpose of this paper is to analyze collective effects by systematically laying out a method for analyzing the stability of a particle beam. This is done by finding a selfconsistent beam distribution, then determining if perturbations to that distribution grow exponentially. This type of analysis has been done in the past for a single bunch, as well as for beams having multiple bunches. However, these analyses have typically been done in different ways-for a single bunch, oscillation modes of the bunch are generally considered to be coupled to one another. The modes of multiple bunches with different oscillation modes are generally taken to be uncoupled, however. In this dissertation, the approaches are unified so that the multibunch modes are all considered to be coupled to one another.

Chapter 2 will describe the storage ring and the types of beam oscillations studied here, and will describe why effects are expected from the coupling of multibunch modes. Chapter 3 will then mathematically describe the interaction between the particle beam and its environment. The Vlasov equation is introduced at this point, which is the equation describing the time evolution of the beam. Chapter 4 will show how the Vlasov equation can be turned into an eigenvalue-like equation for a eigenfrequency $\Omega$. The imaginary part of $\Omega$ will determine if the system is stable. Finally, chapter 5 will show the phenomena that result from including coupling of multibunch modes to one another. The PEP-II $B$ factory will be used as an example for this purpose.

This dissertation demonstrates that under appropriate conditions, multibunch mode coupling can be a significant effect. Machines are being built which begin to approach conditions where this could be a problem. In the process, an approach to the study of collective effects has been developed which is more systematic and complete than has been done in the past. 
time. Then the time offset $\tau$ at a point $s$ in the ring is the time of arrival of the particle at the position $s$ minus the time of arrival of the synchronous particle at the same $s$.

\section{Chapter 2}

\section{Description of the Physical System}

\subsection{The Storage Ring}

\subsubsection{Coordinate System}

Begin by considering a closed, planar curve $C$ which does not intersect itself. This curve is the ideal orbit of a particle in the storage ring. A single particle whose energy is equal to the "ideal energy" will follow this curve if it is started on the curve with velocity tangential to the curve. The particle is confined to this curve by a series of magnets as described later. The coordinates of a point $P$ in relation to the curve $C$ are defined as follows:

- The minimum distance from $P$ to the plane of the curve $C$ will be called $y$. One may choose the positive direction for this coordinate arbitrarily, but it is generally taken to be positive in the "upward" direction.

- Consider the point $P_{1}$ which is the point in the plane of the curve $C$ which is closest to $P$. The minimum distance from $P_{1}$ to $C$ will be called $x$. This value will be positive if it is outside of the curve, negative if it is inside.

- The curve $C$ is parameterized by its arc length. The value of the arc length of the point on $C$ which is closest to $P_{1}$ will be called $s$. The direction of parameterization of $C$ is chosen such that the unit vectors corresponding to $x, s$, and $y$ respectively form a right-handed coordinate system.

It will also be useful to define an arrival time offset $\tau$ as follows: define a "synchronous particle" as one which follows exactly the curve $C$, and starts its motion at an arbitrary

\subsubsection{Forces on a Particle}

A single particle feels forces due to three different effects. First, there are magnets which are designed to keep the particles confined near the ideal orbit (the curve $C$ described above). The fields in these magnets are not time varying. Since the velocity of the particles is very close to the speed of light, and since the particles remain very close to the ideal orbit, the magnetic field in the $s$ ("longitudinal") direction has a negligible effect compared to the magnetic field in the other perpendicular ("transverse") directions. Since the magnetic fields are time independent and the longitudinal magnetic fields are being ignored, the magnetic field can be described by a magnetic vector potential with no transverse components.

The second source of forces on the charged particle are of cavities. These cavities are designed to keep the energy of the particles near the design energy and to keep the particles in bunches. They do this by using an electric field which is varying sinusoidally in time. The phase offset of the sinusoidal oscillations is such that a particle which arrives exactly on time gains exactly the energy it lost on the previous turn due to synchrotron radiation. The slope of the electric field with respect to time is such that a particle which is in the tail of the bunch will have its energy changed so as to move it forward in the bunch, and vice-versa. This electric field can be represented by a longitudinal magnetic vector potential which has no transverse dependence.

The third source of forces on the charged particles are wakefields. These are electromagnetic fields which are generated by particles which are (usually) in front of the particle in question, and act back on that particle. The existence of such fields is due to the fact that the particles are moving within a vacuum chamber which in general is not smooth or perfectly conducting. Currents persist in the vacuum chamber walls for a time after the source particles pass, and thus can cause fields which act back on later particles.

\subsubsection{Feedback}

As will be seen later, bunches which are coupled together by wakefields tend to oscillate unstably if the wakefields are strong enough to make it from one bunch to the next. One way of controlling such an oscillation is with a feedback system. The principle behind a 
feedback system is to determine the bunch's position at one point (the "pickup"), and to apply an impulse at a later point (the "kicker") so as to cancel the oscillation amplitude of the beam (or possibly shift the frequency of coherent oscillations). Some assumption is made about the particle motion between the pickup and kicker so as to determine the relationship between the signal at the pickup and the impulse to be applied at the kicker.

\subsection{Oscillation Modes of the Beam}

The beam consists of several bunches of charged particles which are kept in bunches longitudinally by the rf cavities and transversely by the magnets as described above. If these were the only forces on the beam, analysis of the nonlinear dynamics of a single particle due to these forces would be sufficient to determine the stability of the beam.

However, the wake forces described above cause the the motion of one particle to affect the motion of other particles. Since all the particles are thus coupled to one another, the entire beam will execute coherent oscillations. Such oscillations may or may not be stable. The task of this dissertation is to describe a method for determining the stability of these oscillations under certain circumstances.

\subsubsection{Oscillations without Wakefields}

Consider a single bunch of particles, in the absence of wakefields. The distribution function at a given time for a single bunch can be written in terms of the action-angle variables of the Hamiltonian system described by the magnets and rf cavities (see section 3.2.5) as

$$
\Psi(J, \theta)=\sum_{m} \Psi_{m}(J) e^{i m \cdot \theta} .
$$

The Vlasov equation (see section 3.3 ) says that the time evolution of this distribution is

$$
\Psi(J, \theta, t)=\sum_{m} \Psi_{m}(J) e^{i m \cdot(\theta-\omega t)},
$$

where the components of $\boldsymbol{\omega}$ are the oscillation frequencies for quasi-periodic motion of a single particle. The Fourier modes $\Psi_{m}$ for $m \neq 0$ are generally perturbations about $\Psi_{0}(J)$. Writing the vector $m$ as $\left(m_{y}, m_{\tau}\right)$ (the components in the vertical and longitudinal directions), the modes which are driven most strongly by wakefields are usually the modes where $m_{y}=0$ and where $m_{y}=1$. These are known as longitudinal and transverse modes, respectively (the horizontal transverse modes have been ignored; they parallel the vertical ones). $m_{\tau}$ will be used to refer to the mode, and will simply be referred to as $m$. The sign of $m_{r}$ will be ignored; $m$ will always be positive. Thus, for instance, the $m=0$ transverse mode oscillates rigidly up and down (or horizontally) (see figure 2.1), while the $m=1$ oscillation is one where the head and the tail of the bunch are oscillating oppositely (figure 2.2). Similarly, the $m=1$ longitudinal mode oscillates back and forth rigidly (figure 2.3), while for the $m=2$ longitudinal mode, charge switches from being at the center of the bunch to being at the two ends of the bunch (figure 2.4).
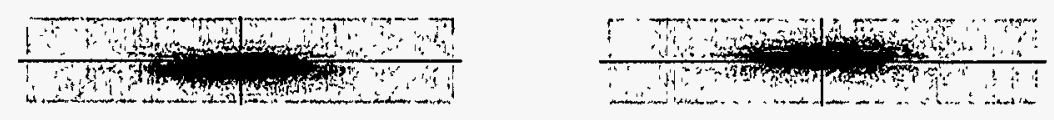

Figure 2.1: Transverse $m=0$ (rigid) oscillation, perturbing a $\Psi_{0}$

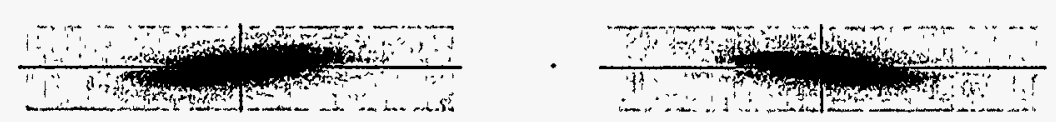

Figure 2.2: Transverse $m=1$ (head-tail) oscillation, perturbing a $\Psi_{0}$.
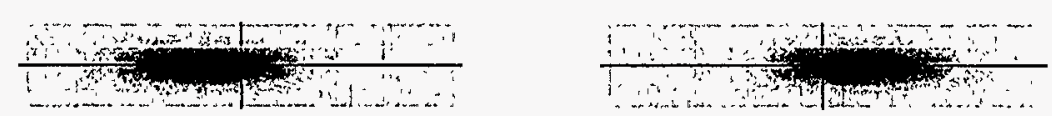

Figure 2.3: Longitudinal $m=1$ (rigid) oscillation, perturbing a $\Psi_{0}$.

Equation (2.2) shows that the oscillation frequency of the $m$ mode is $m \cdot \omega$. If an observer at a fixed position with respect to the ring observers the frequency spectrum of the bunch current, they will see the frequency $m \cdot \omega$ plus all multiples of $\omega_{0}$, since the bunch passes by once every $2 \pi / \omega_{0}$.

A "multibunch mode" is a mode where every bunch in the ring is executing the same type of oscillation (basically, the same $m$ for every bunch). The observer at a fixed position with respect to the ring will see, if there are $M$ identical bunches symmetrically placed around the ring, a frequency spectrum for a single multibunch mode which contains $m \cdot \omega$ 

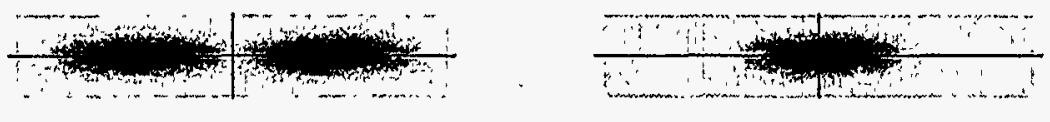

Figure 2.4: Longitudinal $m=2$ oscillation, perturbing a $\Psi_{0}$.

(corresponding to the motion of the bunches with respect to their unperturbed orbits), an offset $p_{0} \omega_{0}$, where $p_{0}$ is an integer, and all multiples of $M \omega_{0}$ offset from that frequency. The reason for the lines being separated by $M \omega_{0}$ is that once every $2 \pi / M \omega_{0}$, a bunch will pass by. The offset $p_{0} \omega_{0}$ is due to the fact that since a given bunch must pass by once every $2 \pi / \omega_{0}$, the spectrum must be offset by some integer multiple of $\omega_{0}$. The value of $p_{0}$ is determined by the phase relation of the single-bunch oscillations with one another.

\subsubsection{Single Bunch Mode Coupling}

Single bunch mode coupling is an effect where two modes whose oscillations are stable for low currents become unstable at higher currents. This instability occurs at the point where the frequencies of the two modes are identical. This instability is essentially a resonant driving by the modes of each other. Resonant driving requires two things to happen: the frequencies of the modes must be identical, and there must be some method of coupling the two modes together so that they can drive each other.

These two effects occur in a single bunch. Consider a single bunch of particles, and for now ignore the possibility that a wakefield generated by the bunch will affect the bunch on the next turn. Consider transverse oscillations; longitudinal oscillations follow a similar argument. The bunch will see an average wakefield due to itself. It turns out that for sufficiently short bunches, that average wakefield will give a force in the same direction as the bunch's displacement [11]. This causes the oscillation frequency of the $m=0$ mode to be reduced from the frequency it had without wakefields. For sufficiently large currents, the frequency of this mode will decrease to the point where it is equal to that of one of the $m=1$ modes.

Now, consider that $m=0$ mode oscillation in more detail. The front of the bunch will see no wakefield. The tail of the bunch, however, will see the wakefield from all of the bunch. As can be seen from figure 2.2, this distribution of force will drive an $m=1$ mode. Thus, there is a mechanism for the $m=0$ mode to drive an $m=1$ mode.
Therefore, the two pieces necessary to get resonant driving occur in a single bunch, and give single bunch mode coupling. Single-bunch mode coupling is a well understood phenomenon, and has been studied by many authors $[38,55,57,58,60,63,65]$.

\subsubsection{Multibunch Mode Coupling}

Now, consider a ring containing several bunches. Further, assume that there are wakefields which remain longer than the distance between two bunches. In addition to the effect of a single bunch on itself, there is now an effect of a bunch on some or all of the other bunches.

If only a single type of multibunch mode is considered (i.e., all bunches with the same $m$ ), then the results are well known, at least in the case of symmetric bunches (identical bunches evenly spaced around the ring) $[40,59,60,67,70]$.

Oscillation modes in the ring are driven when the frequency spectrum of the oscillation mode couples to the frequency spectrum of the wakefields. The frequency spectrum of the wakefields is called the impedance. Single bunch modes essentially average over the impedance, since their mode spectrum consists of closely spaced lines (as described above). The only exception is for extremely long-range wakefields, which last for several turns. Such wakefields cause the Robinson instability [11] and similar instabilities. Multibunch modes, however, have a frequency spectrum which is widely spaced, as described above. Thus, they tend to drive and be driven by small parts of the impedance spectrum; each multibunch mode with a different $p_{0}$ is driven differently. The difference between the driving of different multibunch modes is relevant only when there are certain frequencies for which the impedance takes on values significantly different from its value at nearby frequencies. The impedance will differ significantly from the average behavior around it ("narrow-band impedance") only for wakefields which extend from one bunch to the next. Thus, multibunch modes will only differ in behavior from single bunch modes when there are wakefields extending from one bunch to the next.

The number of multibunch modes corresponding to each single bunch mode is equal to the number of bunches in the ring. These modes will have frequency shifts which are both larger and smaller than the frequency shift for the corresponding single bunch mode. The average of the frequency shifts for all the modes is approximately equal to the single bunch frequency shift. Thus, for some of the multibunch modes, the current at which the mode frequencies coincide can potentially be significantly lower than the current at which the single bunch mode frequencies coincided. Also, the multibunch modes have nonzero 
growth rates, so that the frequencies of the modes are no longer sharp, but acquire a width corresponding to the imaginary part of the mode frequency. This further lowers the current at which effects of coupling between multibunch modes is expected.

Consider transverse multibunch oscillations. An $m=0$ multibunch oscillation induces a wakefield which in general has a nonzero slope at each bunch (even if the wakefield a bunch sees due to itself is ignored). Thus, an $m=0$ multibunch mode can potentially drive an $m=1$ multibunch mode even more strongly than if there were only a single bunch.

Thus, it is expected that multibunch modes will have an effect similar to single-bunch mode coupling, but that it will occur at lower currents and be stronger.

\section{Chapter 3}

\section{Mathematical Description of the}

\section{System}

This chapter mathematically describes the physical system being studied in this dissertation. It will lay out the equations that need to be solved, as well as the components that go into those equations. The next chapter discusses the solution of the equations.

This chapter begins by describing the wake forces that one particle has on another, and showing how such forces are approximated mathematically. The next section shows how the total force on a given particle is described in terms of a Hamiltonian, including the effects of wakefields as they are described in the first section. Finally, the Vlasov equation is introduced, which is the equation which will be used to determine the stability of a distribution of particles. The Vlasov equation requires the Hamiltonian described in this chapter to be inserted into it.

\subsection{Wake Functions}

As described above, a test charge feels a force due to the other particles in the beam. This force comes about because currents persist in the vacuum chamber walls after a particle passes. This will be called a wake response. This effect is modelled by assuming that when a particle passes a given longitudinal position $s$, it generates fields which only affect other particles when they pass that same longitudinal position $s$. This is certainly a good approximation for devices such as rf cavities where the wakefields are a result of trapped modes in the cavity. 
In reality, the objects in the ring that generate strong wakefields have a finite length. For such an object, the most straightforward approach is to consider the change in momentum of a particle that passes through the object. Since electromagnetic fields from the object should affect a particle only for a finite interval of $s$, this change in momentum is well defined. This change in momentum is then averaged over the length of the object in some appropriate way to fit the behavior into the model described in the previous paragraph.

This section begins by describing, under certain approximations appropriate for highenergy storage rings, the force that a test charge can feel. Then, the types of fields that a test charge can generate are described. The next subsection shows how these two facts can be combined to show the general form for the force that a distribution can cause on a test charge. Wakefields are formally defined here, and the impedance is defined in terms of them.

\subsubsection{Force on a Test Charge}

To determine the response of a test charge to a source distribution, the change in momentum of a particle passing through an object containing electromagnetic fields must be described. Begin with Maxwell's equations and the Lorentz force law

$$
\begin{array}{rr}
\nabla \cdot E=\rho & \nabla \times E=-\frac{1}{c} \frac{\partial B}{\partial t} \\
\nabla \cdot B=0 & \nabla \times B=\frac{1}{c} \frac{\partial E}{\partial t}+\frac{4 \pi}{c} J \\
& F=e\left(E+\frac{v}{c} \times B\right) .
\end{array}
$$

Here $E$ is the electric field, $B$ is the magnetic field, $\rho$ is the charge density, $J$ is the current density, $c$ is the speed of light, and $e$ and $v$ are the charge and velocity of the particle feeling the force $F$. Note that here and throughout this dissertation, Gaussian units will be used.

The test charge is in free space; thus $\rho=0$ and $J=0$. First of all, it is useful to take the curl of $F$ :

$$
\nabla \times F=-\frac{e}{c}\left[\frac{\partial B}{\partial t}+(v \cdot \nabla) B\right]
$$

If a particle is following a trajectory $x=X\left(x_{0}, t\right)$, where $X$ is such that $X\left(x_{0}, 0\right)=x_{0}$ and $(\partial / \partial t) X\left(x_{0}, t\right)=v$, then the bracketed piece of equation (3.4) is the total time derivative of $B$ as seen by a particle following this trajectory. Thus, the time integral along such a trajectory of $\nabla \times F$ is

$$
\int_{t_{0}}^{t_{1}} d t(\nabla \times F)_{x=X\left(x_{0}, t\right)}=-\frac{e}{c}\left[B\left(X\left(x_{0}, t_{1}\right), t_{1}\right)-B\left(X\left(x_{0}, t_{0}\right), t_{0}\right)\right]
$$

Thus, if a particle begins and ends in a region with no magnetic field, this integral is zero. It is expected that this will be the case, under the assumption that the fields generated at an object will be confined to the region of the object.

Next, compute the divergence of the component of $F$ transverse to the velocity $v$ :

$$
\begin{gathered}
\nabla \cdot F_{\perp}=-\frac{e}{c^{2}}\left[\frac{\partial}{\partial t}+(v \cdot \nabla)\right](v \cdot E)+\frac{e}{c^{2}} \frac{\partial v}{\partial t} \cdot E-\frac{1}{\gamma_{0}^{2}} \nabla \cdot F_{\|} \\
F_{\|}=\frac{v \cdot F}{v \cdot v} v \quad F_{\perp}=F-F_{\|} \quad \gamma_{0}=\frac{1}{\sqrt{1-v ; v / c^{2}}} .
\end{gathered}
$$

This can also be integrated with respect to time along a particle trajectory which begins and ends in a field-free region; the result is

$$
\int_{t_{0}}^{t_{1}} d t\left[\nabla \cdot\left(F_{\perp}+\frac{1}{\gamma_{0}^{2}} F_{\|}\right)-\frac{e}{c^{2}} \frac{\partial v}{\partial t} \cdot E\right]_{x=X\left(x_{0}, t\right)}=0
$$

Next, for the purposes of doing the above integrations, assume that the velocity of a test charge is constant through the object. As long as the object is relatively short, this should be a good approximation. In that case, $X\left(x_{0}, t\right)=x_{0}+v t$. Also, the change in momentum imparted to the test particle will be

$$
\Delta p\left(x_{0}\right)=\int_{t_{0}}^{t_{1}} d t F_{x=X\left(x_{0}, t\right)} .
$$

Combining these results with equations (3.5) and (3.8) gives

$$
\nabla_{0} \times \Delta p=0 \quad \nabla_{0} \cdot\left(\Delta p_{\perp}+\frac{1}{\gamma_{0}^{2}} \Delta p_{\|}\right)=0,
$$

where $\nabla_{0}$ takes derivatives with respect to $x_{0}$, and $\Delta p_{\perp}$ and $\Delta p_{\|}$are defined from $\Delta p$ analogously to $F_{\perp}$ and $F_{\|}$.

The first of the equations in (3.10) says that $\Delta p$ can be written as $\Delta p\left(x_{0}\right)=-e \nabla f$ for some scalar function $f$. For convenience, define cylindrical coordinates with $z$ being parallel to $v$, and write $x_{0}$ in these coordinates. Then the second of the equations in (3.10) gives the differential equation

$$
\frac{1}{r} \frac{\partial}{\partial r}\left(r \frac{\partial f}{\partial r}\right)+\frac{1}{r^{2}} \frac{\partial^{2} f}{\partial \phi^{2}}+\frac{1}{\gamma_{0}^{2}} \frac{\partial^{2} f}{\partial z^{2}}=0
$$


Assuming the solutions to be bounded for large $z$ gives the solution

$$
f(r, \phi, z)=\sum_{m} \int_{-\infty}^{\infty} A_{m}(k) I_{m}\left(\frac{k r}{\gamma_{0}}\right) e^{i(m \phi+k z)} d k,
$$

where $I_{m}$ is a modified Bessel function. Note that $\left[A_{m}(k)\right]^{*}=A_{-m}\left(-k^{*}\right)(-1)^{m}$, since $f$ is real. If $A_{m}(k)$ is significant only for $k \ll \gamma_{0} / r$, then equation (3.12) can be written as

$$
f(r, \phi, z)=\sum_{m} \hat{A}_{m}(z) r^{m} e^{i m \phi} .
$$

Again, $\hat{A}_{m}^{*}(z)=\hat{A}_{-m}(z)$ from the reality of $f$. An index $m$ has been introduced here; it differs from the $m$ defined in section 2.2.1. The $m$ used here refers to the azimuthal distribution in real space of charge. The $m$ defined in section 2.2.I refers to the azimuthal dependence of the distribution in longitudinal phase space.

The approximation that $k \ll \gamma_{0} / r$ is generally excellent; for example, for $2.5 \mathrm{GeV}$ electrons and a radius of $1 \mathrm{~cm}$, this amounts to ignoring frequencies above $20 \mathrm{THz}$ ! Another way of looking at this approximation is to consider that frequencies that are much larger than the bunch length will tend to have no average effect on the bunch. Thus, the condition becomes $\gamma_{0} \sigma_{\ell} \gg \sigma_{r}$, where $\sigma_{r}$ is a typical transverse size of the bunch, and $\sigma_{\ell}$ is the bunch length. Using PEP-II parameters [49], the ratio $\gamma_{0} \sigma_{\ell} / \sigma_{r}$ is at worst about 17500 , and thus $k \ll \gamma_{0} / r$ is an excellent approximation. It should be about as good an approximation in other storage ring designs.

\subsubsection{Fields of a Source Distribution}

The next step in determining the test charge's response to a source distribution is to determine the electromagnetic fields generated by a source distribution. These fields will induce currents and charges in the vacuum chamber walls, which can persist beyond the time that the source distribution passes.

Begin with the fields generated by a point charge in free space. These are computed by giving a Lorentz boost to the fields of a stationary point charge with charge $q$ :

$$
\begin{gathered}
E_{r}=\frac{B_{\phi}}{\beta_{0}}=\frac{\gamma_{0} g r}{\left[r^{2}+\gamma_{0}^{2}\left(z-\beta_{0} c t\right)^{2}\right]^{3 / 2}} \\
E_{z}=\frac{\gamma_{0} q\left(z-\beta_{0} c t\right)}{\left[r^{2}+\gamma_{0}^{2}\left(z-\beta_{0} c t\right)^{2}\right]^{3 / 2}} .
\end{gathered}
$$

The fields have been written in cylindrical coordinates with the $z$ direction being parallel to the charge's velocity, which is $\beta_{0} c$.

Now assume that there is an infinitely massive test particle with a velocity of $\beta_{r} c \hat{r}$ (it has no velocity in the $z$ direction) and charge $e$. This is a fair assumption since the charges in the vacuum chamber walls are moving slowly relative to the source distribution. The test particle is at radius $r$ and $z=0$ at time $t=0$. The source charge imparts a momentum to it of

$$
\Delta p=\frac{2 q e}{r c \sqrt{\beta_{r}^{2}+\beta_{0}^{2} \gamma_{0}^{2}}}\left[\gamma_{0} \hat{r}+\beta_{r} \frac{\gamma_{0}^{2} \beta_{0}^{2}+1}{\gamma_{0} \beta_{0}} \hat{z}\right] .
$$

In the limit where $\gamma_{0} \beta_{0} \gg 1$ (certainly valid for high energy rings), this expression becomes

$$
\Delta p \rightarrow \frac{2 q e}{r c \beta_{0}}\left(\hat{r}+\beta_{r} \beta_{0} \hat{z}\right) .
$$

This is the momentum that would be imparted to a test charge by the fields

$$
E_{r}=\frac{B_{\phi}}{\beta_{0}}=\frac{2 q}{r} \delta\left(z-\beta_{0} c t\right) \quad E_{z}=0,
$$

where $\delta$ is the Dirac delta function. These are exactly the fields due to an infinite line charge, except for the delta function factor.

Using the fields from equation (3.18), the electromagnetic fields from a charge density $\rho\left(r, \phi, z-\beta_{0} c t\right)$ can be computed. The electric field is given by the integral

$$
\boldsymbol{E}(r, \phi, z, t)=2 \int \rho\left(r^{\prime}, \phi+\phi^{\prime}, z-\beta_{0} c t\right) \frac{\left(r-r^{\prime} \cos \phi^{\prime}\right) \hat{r}-r^{\prime} \sin \phi^{\prime} \hat{\phi}}{r^{2}+r^{\prime 2}-2 r r^{\prime} \cos \phi^{\prime}} r^{\prime} d r^{\prime} d \phi^{\prime} .
$$

The magnetic field is $B=\beta_{0} \hat{z} \times E$. $\rho$ can be written as

$$
\rho(r, \phi, z)=\sum_{m} \rho_{m}(r, z) e^{i m \phi}
$$

Note that $\rho_{-m}(r, z)=\rho_{m}^{*}(r, z)$. Then the.integral (3.19) can be performed:

$$
\begin{aligned}
E_{r}(r, \phi, z, t)=\frac{B_{\phi}(r, \phi, z, t)}{\beta_{0}}= & 4 \pi\left[\sum_{m=0}^{\infty} \int_{0}^{r} \Re\left\{\rho_{m}\left(r^{\prime}, z-\beta_{0} c t\right) e^{i m \phi}\right\}\left(\frac{r^{\prime}}{r}\right)^{m+1} d r^{\prime}\right. \\
& \left.-\sum_{m=1}^{\infty} \int_{r}^{\infty} \Re\left\{\rho_{m}\left(r^{\prime}, z-\beta_{0} c t\right) e^{i m \phi}\right\}\left(\frac{r}{r^{\prime}}\right)^{m-1} d r^{\prime}\right]
\end{aligned}
$$




$$
\begin{aligned}
E_{\phi}(r, \phi, z, t)=-\frac{B_{r}(r, \phi, z, t)}{\beta_{0}}= & 4 \pi \sum_{m=1}^{\infty}\left[\int_{0}^{r} \Im\left\{\rho_{m}\left(r^{\prime}, z-\beta_{0} c t\right) e^{i m \phi}\right\}\left(\frac{r^{\prime}}{r}\right)^{m+1} d r^{\prime}\right. \\
& \left.+\int_{r}^{\infty} \Im\left\{\rho_{m}\left(r^{\prime}, z-\beta_{0} c t\right) e^{i m \phi}\right\}\left(\frac{r}{r^{\prime}}\right)^{m-1} d r^{\prime}\right]
\end{aligned}
$$

Consider a source distribution moving inside a vacuum chamber. Since the source distribution is affecting the vacuum chamber walls, which are outside of the distribution, only the first integral in equations (3.21) and (3.22) is nonzero in the region of interest.

\subsubsection{Wake Response to the Source}

The results of sections 3.1.1 and 3.1.2 can be combined to give the response of a test charge to a source distribution. Since the source distribution and test charge are both moving with velocities very close to the speed of light, the $z$ axes of sections 3.1.1 and 3.1.2 can be chosen to be the same (and, on average at least, parallel to the distributions' velocities). Consider a system that is cylindrically symmetric about this $z$ axis.

From equation (3.21), a distribution proportional to $\cos m \phi$ produces fields $E_{r}$ and $B_{\phi}$ proportional to $\cos m \phi$, and $E_{\phi}$ and $B_{r}$ proportional to $\sin m \phi$. Examination of Maxwell's equations (3.1) and (3.2) shows that these fields will induce, in the vacuum chamber walls, a charge density $\rho$ and current densities $J_{r}$ and $J_{z}$ proportional to $\cos m \phi$, and a current density $J_{\phi}$ proportional to $\sin m \phi$. These will in turn induce back in the chamber fields $E_{r}, B_{\phi}$, and $E_{z}$ proportional to $\cos m \phi$, and $E_{\phi}, B_{r}$, and $B_{z}$ proportional to $\sin m \phi$. From the discussion in section 3.1.1, it follows that such fields can only result in an $f$ (as defined at the end of section 3.1.1) which is also proportional to $\cos m \phi$. It thus follows from the linearity of Maxwell's equations that a Fourier mode $\rho_{m}\left(r^{\prime}, z^{\prime}\right)$ of the charge density will cause a momentum kick to a subsequent test particle which results from an $f$ which has an $\hat{A}_{m}(z)$ which is a linear combination of

$$
\lambda_{m}\left(z^{\prime}\right)=2 \pi \int_{0}^{\infty} \rho_{m}\left(r^{\prime}, z^{\prime}\right) r^{m+1} d r^{\prime}
$$

for various $z^{\prime}$.

As can be seen from the fact that $\rho$ was defined to depend on $z-\beta_{0} c t$, the $z^{\prime}$ in $\rho_{m}\left(r^{\prime}, z^{\prime}\right)$ refers to the position in the ring at time $t=0$ of a slice of the distribution. Also, as described in section 3.1.1, the argument $z$ of $f$ refers to the position of a test particle at time $t=0$. If the object causing a wake response is at position $s$ in the ring, then the arrival time $t$ of a particle at that object is given by the formula $s=z+\beta_{0} c t$, where $z$ is the value of $s$ at $t=0$. The reference particle described in the definition of the time offset $\tau$ in section 2.1.1 arrives at the point $s$ at time $t_{\mathrm{ref}}$ given by $s=z_{\mathrm{ref}}+\beta_{0} c t_{\mathrm{ref}}$. Thus, there is a simple linear relation between the time offset $\tau$ and the initial condition $z: z=z_{\text {ref }}-\beta_{0} c \tau$. The number $z_{\text {ref }}$ depends only on the position of the object whose wake response in being computed. Thus, the initial condition $z$ and the time offset $\tau$ are interchangeable ways of parameterizing these functions (although one must be careful about taking derivatives). Thus, define $\lambda_{m}(\tau)=\beta_{0} c \lambda_{m}(z)$ and $f(r, \phi, \tau)=f(r, \phi, z)$, where $z$ and $\tau$ are related as described above.

Finally, since external forces are not being considered here, the system should be time invariant. Thus, the wake response should only depend on the difference in the arrival times of the source and test particles at the object whose wake response is being determined. Equivalently, if the $f$ that gives the momentum kick is parameterized by $\tau$, and the source distribution is parameterized by $\tau^{\prime}$, the proportionality constant will only depend on $\tau-\tau^{\prime}$. Thus, the function $f$ can be written in terms of the source distribution as

$$
f(r, \phi, \tau)=\sum_{m} r^{m} e^{i m \phi} \int_{-\infty}^{\infty} W_{m}\left(\tau-\tau^{\prime}\right) \lambda_{m}\left(\tau^{\prime}\right) d \tau^{\prime}
$$

Thus, the wake response due to a cylindrically symmetric object can be completely characterized by the set of functions $W_{m}(\tau)$, known as wake functions.

Since the ring consists of several objects at various locations, each of which will have a different wake function, wake functions for the entire ring $W_{m}(\tau, s)$ can be defined as follows: for each object with a wake response, determine its wake functions $W_{m}(\tau)$. Define $W_{m}(\tau, s)$ for that object to be zero for $s$ outside of the range of action of the object, and integrated over the length of the object such that $\int W_{m}(\tau, s) d s=\beta_{0} c W_{m}(\tau)$. Then add together all the $W_{m}(\tau, s)$ for all the objects in the ring. Note that $f(r, \phi, \tau)$ thus becomes $f(r, \phi, \tau, s)$ :

$$
f(r, \phi, \tau, s)=\sum_{m} r^{m} e^{i m \phi} \int_{-\infty}^{\infty} W_{m}\left(\tau-\tau^{\prime}, s\right) \lambda_{m}\left(\tau^{\prime}\right) d \tau^{\prime} .
$$

The integral of $W_{m}(\tau, s)$ over $s$ around the ring is consistent with the $W_{m}(z)$ in [11] if $z=-\beta_{0} c r$.

To simplify notation, $W_{1}$ is written as $W_{\perp}$, since the contribution of the $W_{1}$ wake usually dominates the transverse wake force. 


\subsubsection{Impedance}

The impedance is defined to be the Fourier transform of the wake function. The transverse impedance will be defined as

$$
Z_{m}^{\frac{1}{m}}(\omega, s)=i \int_{-\infty}^{\infty} W_{m}(\tau, s) e^{i \omega \tau} d \tau
$$

The longitudinal impedance is defined to be

$$
Z_{m}^{\|}(\omega, s)=-\frac{1}{\beta_{0} c} \int_{-\infty}^{\infty} W_{m}^{\prime}(\tau, s) e^{i \omega \tau} d \tau
$$

The integral of these quantities over $s$ around the ring are consistent with the $Z_{\frac{1}{1}}^{1}$ and $Z_{m}^{\|}$ in [11]. Note that integrating by parts leads to $Z_{m}^{\|}(\omega, s)=\omega Z_{m}^{\frac{1}{m}}(\omega, s) / \beta_{0} c$. This is a minor variation on what is known as the Panofsky-Wentzel theorem [11].

Note that $\left[Z_{m}^{\perp}\left(-\omega^{*}\right)\right]^{*}=-Z_{m}^{\frac{1}{m}}(\omega)$, and $\left[Z_{m}^{\|}\left(-\omega^{*}\right)\right]^{*}=Z_{m}^{\|}(\omega)$, since $W_{m}$ is real.

$Z_{0}^{\|}$is written as $Z_{\|}$, since it usually dominates the longitudinal wake force. Similarly, $Z_{\perp}^{\perp}$ is written as $Z_{\perp}$, since it usually dominates the transverse wake force.

\subsection{Hamiltonian for a Single Particle}

3.2.1 General Electromagnetic Hamiltonian for a Storage Ring

The Hamiltonian for a charged particle moving in an external electromagnetic field is [37]

$$
H=c \sqrt{\left(p-\frac{e}{c} A(x, t)\right)^{2}+(m c)^{2}}+e \Phi(x, t)+V(x, t) .
$$

where $\boldsymbol{x}$ and $\boldsymbol{p}$ are the canonical position and momentum, respectively, $V$ is an external potential, and $\Phi$ and $A$ are the scalar and vector electromagnetic potentials respectively. $m$ and $e$ are the mass and charge of the particles in question.

As described in section 2.1.2, the forces due to the rf cavities and the magnets that focus the beam can both be described through a vector potential which only has a component in the $s$ direction. The discussion in section 3.1 .1 shows that the wake force can be described through the external potential $V$. The Hamiltonian under these assumptions thus looks like

$$
H=c \sqrt{p_{x}^{2}+p_{y}^{2}+\left(\frac{p_{s}}{1+h(s) x}-\frac{e}{c} A_{s}(x, t)\right)^{2}+(m c)^{2}}+e f,
$$

where $1 / h$ is the local radius of curvature of the ideal orbit $C$, and $f$ is the function defined in equation (3.25). The arguments of $f$ have been ignored for now; they will be included once the Hamiltonian has been simplified somewhat.

The Hamiltonian is next converted to a form where $s$ is the independent variable. This is made possible by the fact that $s$ is a monotonically increasing function of $t$. One would expect that to do this, one would have to take Hamilton's equations, turn the $t$ derivatives into $s$ derivatives, and find a Hamiltonian that generates the resulting equations. It turns out, however, that all one bas to do is to take the Hamiltonian (3.29) and solve for $-p_{s}$ $[19,43]$ :

$$
-p_{s}=-[1+h(s) x] \frac{e}{c} A_{s}(x, t) \pm[1+h(s) x] \sqrt{\left(\frac{H}{c}-\frac{e}{c} f\right)^{2}-p_{x}^{2}-p_{y}^{2}-(m c)^{2}} .
$$

Note that the momentum conjugate to $t$ is $5 H$. The sign in equation (3.30) is determined by considering a particle which follows the reference orbit $C$. Hamilton's equations give the equation

$$
\frac{d t}{d s}=\mp \frac{H}{\sqrt{H^{2}-\left(m c^{2}\right)^{2}}}
$$

along the reference orbit when $f=0$. Equation (3.29) shows that $H>0$. Thus, if the particle is moving in the positive $s$ direction, the bottom sign should be chosen. This convention will be used henceforth.

Finally, it is useful to change variables to

$$
\tau=t-\frac{s}{\beta_{0} c} \quad \delta=\gamma_{0} m c^{2}-H .
$$

Note that $\tau$ is the time offset described in section 2.1.1. The term $\delta / \beta_{0} c$ is subtracted from the Hamiltonian because of this transformation, as described in appendix A.1. Next, the Hamiltonian and the momenta $p_{x}, p_{y}$, and $\delta$ are all scaled by $p_{0} \equiv \beta_{0} \gamma_{0} m c$. The resulting Hamiltonian is dimensionless, and is given by

$$
\begin{aligned}
-p_{s}=-[1+h(s) x] \frac{e}{p_{0} c} A_{s}\left(x, \tau+\frac{s}{\beta_{0} c}\right)-\frac{\delta}{\beta_{0} c} \\
-[1+h(s) x] \sqrt{\left[\frac{1}{\beta_{0}}-\frac{\delta}{c}-\frac{e}{p_{0} c} f(x, y, \tau, s)\right]^{2}-p_{x}^{2}-p_{y}^{2}-\left(\frac{m c}{p_{0}}\right)^{2}} .
\end{aligned}
$$

The arguments of $f$ have been included at this point; note that the cylindrical coordinates $(r, \phi)$ have been transformed into the locally Cartesian coordinates $(x, y)$, and $z$ has been replaced by $\tau$. The vector $x$ is $(x, s, y)$. 


\subsubsection{Expansion of the Magnetic Field}

Eventually, equation (3.33) will be expanded as a power series in the phase space variables. This requires an expansion of $A_{s}$ for the magnets. Since the magnets are time-independent, $A_{s}$ will not depend on $\tau$. It will depend on $s$, however, since the magnets are localized at various points around the ring. The approach used here is essentially that of [42].

As a first approximation, Maxwell's equations will be solved for $A_{s}$ which is independent of $s$. This is a good approximation in the center of the magnet; the fields on the edges are being ignored. Maxwell's equations are also solved in a region where the radius of curvature $1 / h$ is constant. Thus, Maxwell's equations become

$$
0=\nabla \times \nabla \times A=-\left(\frac{\partial}{\partial x}\left\{\frac{1}{1+h x} \frac{\partial}{\partial x}\left[(1+h x) A_{s}\right]\right\}+\frac{\partial^{2} A_{s}}{\partial y^{2}}\right) \hat{s} .
$$

Expand $A_{s}$ as a power series in $y$ :

$$
A_{s}=\sum_{n=0}^{\infty} \frac{a^{(n)}(x)}{n !} y^{n}
$$

Then equation (3.34) gives a recursion relation for the functions $a^{(n)}$ :

$$
\frac{d}{d x}\left\{\frac{1}{1+h x} \frac{d}{d x}\left[(1+h x) a^{(n)}\right]\right\}=-a^{(n+2)}
$$

This series will terminate only if for some $a^{(n)}$, the left hand side is zero. The null space of the operator

$$
\frac{d}{d x} \frac{1}{1+h x} \frac{d}{d x}(1+h x)
$$

is spanned by the functions

$$
a_{0}(x)=\frac{1}{1+h x} \quad \text { and } \quad a_{1}(x)=\frac{x+h x^{2} / 2}{1+h x} .
$$

These two functions have been chosen such that as $x \rightarrow 0, a_{0}(x) \rightarrow 1$ and $a_{1}(x) \rightarrow x$.

Beginning with $a^{(n)}=a_{0}$ or $a^{(n)}=a_{1}$, the recursion relation (3.36) is solved for the $a^{(k)}$ for which $k<n$. The arbitrary constants resulting from the integrations necessary to solve the recursion relation in this direction can be found by assuming that the behavior of $a^{(k)}$ as $x \rightarrow 0$ goes like $x^{2} a^{(k+2)}$. This results in the lowest order behavior of $A_{s}$ when both $x$ and $y$ are considered being that of a homogeneous polynomial where all of the $a^{(k)}$ are included. Thus, define

$$
a_{n+2}=\frac{1}{1+h x} \int_{0}^{x} d x^{\prime}\left(1+h x^{\prime}\right) \int_{0}^{x^{\prime}} d x^{\prime \prime} a_{n}\left(x^{\prime \prime}\right)
$$

Note that as $x \rightarrow 0, a_{n} \rightarrow x^{n} / n !$. Then $a^{(n-2 k)}=(-1)^{k} a_{2 k+l}$, where $l$ is 0 or 1 depending on where the recursion relation was started. Thus, the solution of (3.34) is given by

$$
\begin{aligned}
& A_{s, N}^{(n)}= \begin{cases}\sum_{k=0}^{n / 2}(-1)^{n / 2-k} a_{2 k+1} \frac{1}{(n-2 k) !} y^{n-2 k} & n \text { even } \\
\sum_{k=0}^{(n+1) / 2}(-1)^{(n+1) / 2-k} a_{2 k} \frac{1}{(n-2 k+1) !} y^{n-2 k+1} & n \text { odd. }\end{cases} \\
& A_{s, S}^{(n)}= \begin{cases}\sum_{k=0}^{n / 2}(-1)^{n / 2-k} a_{2 k} \frac{1}{(n-2 k+1) !} y^{n-2 k+1} & n \text { even } \\
\sum_{k=0}^{(n-1) / 2}(-1)^{(n-1) / 2-k} a_{2 k+1} \frac{1}{(n-2 k) !} y^{n-2 k} & n \text { odd, }\end{cases}
\end{aligned}
$$

where $A_{s, N}^{(n)}$ and $A_{s, S}^{(n)}$ are known as the normal and skew components of the field, respectively. The overall factor has been chosen such that the leading term in $A_{s, N}^{(n)}$ is $x^{n+1} /(n+1) !$, and in $A_{s, S}^{(n)}$ is $y x^{n} / n !$. Table 3.1 gives $a_{n}$ for small values of $n$.

$$
\begin{aligned}
& \text { n } \quad a_{n}(x) \\
& 2 \quad \frac{1}{2 h^{2}}\left[-\frac{h x+(h x)^{2} / 2}{1+h x}+(1+h x) \ln (1+h x)\right] \\
& 3 \text {. } \frac{1}{4 h^{3}}\left[\frac{1}{4}(1+h x)^{3}-\frac{1}{4} \frac{1}{1+h x}-(1+h x) \ln (1+h x)\right] \\
& 4 \frac{1}{8 h^{4}}\left[\frac{1}{8} \frac{1}{1+h x}+\frac{1}{2}(1+h x)-\frac{5}{8}(1+h x)^{3}\right. \\
& \left.+(1+h x) \ln (1+h x)+\frac{1}{2}(1+h x)^{3} \ln (1+h x)\right]
\end{aligned}
$$

Table 3.1: Lowest order values for $a_{n}$.

The primary magnets in an accelerator are bending magnets, quadrupoles, and sextupoles, corresponding to $A_{s, N}^{(0)}, A_{s, N}^{(1)}$, and $A_{s, N}^{(2)}$ respectively. The latter two generally reside in straight sections. The solutions for $A_{s}$ for these three types of magnets are thus

$$
B_{0} x \frac{1+h x / 2}{1+h x} \quad \frac{K}{2} \frac{p_{0} c}{e}\left(x^{2}-y^{2}\right) \quad \frac{S}{6} \frac{p_{0} c}{e}\left(x^{3}-3 x y^{2}\right),
$$

respectively. Note that $h=0$ for the quadrupoles and sextupoles.

See [43] for a different treatment of this. 


\subsubsection{R.F. Cavity Voltage}

There is also a contribution to $A_{s}$ from the rf cavities. This comes from the longitudinal force that a particle feels when passing through the cavity due to the fundamental mode of the cavity which is being externally driven. This fundamental mode is taken to have no transverse dependence, to consist only of a longitudinal electric field, and to be the only mode in the cavity. The change in $\delta$ for a test charge with charge $e$ is then given by

$$
\Delta \delta=-\frac{e}{p_{0}} \int_{-\infty}^{\infty} E_{z}(s) \sin \omega_{\mathrm{rf}}\left(\tau+\frac{s}{\beta_{0} c}\right) d s,
$$

where $\omega_{\mathrm{rf}}$ is the frequency of the fundamental cavity mode. Note that $\omega_{\mathrm{rf}}$ should be an integer multiple of $2 \pi \beta_{0} c / L$, where $L$ is the length of the reference orbit $C$. If this were not the case, the accelerator Hamiltonian would look different from one turn to the next and, among other things, there would be no fixed point of the one-turn map. The integer multiple is denoted by $h_{\mathrm{rf}}$ i.e., $\omega_{\mathrm{rf}}=2 \pi h_{\mathrm{rf}} \beta_{0} c / L$. The integral (3.43) can be written in the form

$$
\Delta \delta=\frac{e \hat{V}}{p_{0}} \sin \left(\omega_{\mathrm{rf}} \tau+\phi_{\mathrm{rf}}\right)
$$

where $\hat{V}$ is the maximum voltage gain by a particle, and $\phi_{\mathrm{rf}}$ is the phase offset of the $\mathrm{rf}$ voltage.

For simplicity, the rf cavity is taken to be infinitesimally thin. Thus, the $A_{s}$ resulting from the rf cavity is

$$
-\hat{V} \frac{c}{\omega_{\mathrm{rf}}} \delta_{L}\left(s-s_{\mathrm{rf}}\right) \cos \left(\omega_{\mathrm{rf}} \tau+\phi_{\mathrm{rf}}\right)
$$

where

$$
\delta_{L}(s)=\sum_{k} \delta(s-k L)
$$

$\delta$ is the Dirac delta function, and $s_{\mathrm{rf}}$ is the position in the ring of the rf cavity.

Note that the voltage is invariant under a translation of $\tau$ by any multiple of $L / \beta_{0} c h_{\mathrm{rf}}$. The ring thus has $h_{\mathrm{rf}}$ "buckets" where a bunch of particles can be. The form of the vector potential has been chosen so that each rf bucket is identical. The coordinate $\tau$ can thus be chosen such that a particle with the ideal energy (i.e., $\delta=0$ ), no transverse displacement, and $\tau=\hat{\tau}_{n 0}=n L / \beta_{0} c h_{\mathrm{rf}}, n$ an integer, should satisfy these same conditions one turn later. If there is only one rf cavity, then this will be true if $\phi_{\mathrm{rf}}=0$. In general, $\phi_{\mathrm{rf}}$ is nonzero, however, since in general there are synchrotron radiation losses, and these must be compensated for by the rf voltage. These synchrotron radiation losses cannot be described by a Hamiltonian, since they are dissipative. Thus, they are ignored in this treatment, and therefore $\phi_{\mathrm{rf}}$ is taken to be 0 .

\subsubsection{Hamiltonian for Small Displacements}

Now, assume that the phase space variables are small quantities. This corresponds to assuming that the particle trajectory is very close to the ideal orbit $C$ and that $\tau$ remains small. The Hamiltonian (3.33) can then be expanded to third order in the phase space variables (fourth order in $\tau$ ), using the results of sections 3.2.2 and 3.2.3. It is only expanded to first order in the wake functions, however, with the coefficient of the wake function expanded in phase space variables only to a first order correction. The result is:

$$
\begin{aligned}
-p_{s}= & \frac{1}{2}\left(p_{x}^{2}+p_{y}^{2}+\frac{\delta^{2}}{\gamma_{0}^{2} \beta_{0}^{2} c^{2}}\right)\left(1+h(s) x+\frac{\delta}{\beta_{0} c}\right)+\frac{h(s) x \delta}{\beta_{0} c}+\frac{1}{2}\left[h^{2}(s)-K(s)\right] x^{2} \\
+ & \frac{1}{2} K(s) y^{2}-\frac{S}{6}\left(x^{3}-3 x y^{2}\right)-\frac{e \hat{V} \omega_{\mathrm{rf}}}{2 p_{0}} \delta_{L}\left(s-s_{\mathrm{rf}}\right) \tau^{2}+\frac{e \hat{V} \omega_{\mathrm{rf}}^{3}}{24 p_{0}} \delta_{L}\left(s-s_{\mathrm{rf}}\right) \tau^{4} \\
& +\frac{e}{\beta_{0} p_{0} c}\left(1+h(s) x+\frac{\delta}{\beta_{0} c \gamma_{0}^{2}}\right) f(x, y, \tau, s)
\end{aligned}
$$

Note that the $B_{0}$ of equation (3.42) has been chosen to be $-h p_{0} c / e$ so as to cancel the term in the Hamiltonian that is linear in $x$. This amounts to choosing the bending field of the magnets to give an on-energy particle a radius of curvature equal to that of the curve $C$ at any given point.

First, consider the terms in the Hamiltonian (3.47) that are quadratic in the phase space variables:

$$
\begin{aligned}
\frac{1}{2} p_{x}^{2}+\frac{1}{2} p_{y}^{2}+\frac{h(s) x \delta}{\beta_{0} c}+\frac{1}{2}\left[h^{2}(s)-K(s)\right] x^{2}+\frac{1}{2} & K(s) y^{2} \\
& +\frac{1}{2} \frac{\delta^{2}}{\gamma_{0}^{2} \beta_{0}^{2} c^{2}}-\frac{e \hat{V} \omega_{\mathrm{rf}}}{2 p_{0}} \delta_{L}\left(s-s_{\mathrm{rf}}\right) \tau^{2} .
\end{aligned}
$$

The first task is to eliminate the term proportional to $x \delta$. This is done by transforming

$$
x \rightarrow x-\eta(s) \frac{\delta}{\beta_{0} c} \quad p_{x} \longrightarrow p_{x}-\xi(s) \frac{\delta}{\beta_{0} c} .
$$


This transformation should be symplectic; thus $r$ must transform as

$$
\tau \rightarrow \tau-\frac{\eta(s)}{\beta_{0} c} p_{x}+\frac{\xi(s)}{\beta_{0} c} x .
$$

The matrix for this transformation can be written as

$$
\left[\begin{array}{cccc}
1 & 0 & 0 & -\eta(s) / \beta_{0} c \\
\xi(s) / \beta_{0} c & 1 & -\eta(s) / \beta_{0} c & 0 \\
0 & 0 & 1 & -\xi(s) / \beta_{0} c \\
0 & 0 & 0 & 1
\end{array}\right] .
$$

Using the results of appendix A.2, the quadratic part of the Hamiltonian becomes

$$
\begin{aligned}
& \frac{1}{2} p_{x}^{2}+\frac{1}{2} p_{y}^{2}+ \frac{1}{2}\left[h^{2}(s)-K(s)\right] x^{2}+\frac{1}{2} K(s) y^{2} \\
&+ \frac{p_{x} \delta}{\beta_{0} c}\left[\frac{d \eta}{d s}-\xi(s)\right]+\frac{x \delta}{\beta_{0} c}\left[h(s)-h^{2}(s) \eta(s)+K(s) \eta(s)-\frac{d \xi}{d s}\right] \\
&+\frac{1}{2} \frac{\delta^{2}}{\beta_{0}^{2} c^{2}}\left\{\xi^{2}(s)+\left[h^{2}(s)-K(s)\right] \eta^{2}(s)+\frac{1}{\gamma_{0}^{2}}+\left[\frac{d \xi}{d s} \eta(s)-\frac{d \eta}{d s} \xi(s)\right]-2 h(s) \eta(s)\right\} \\
&-\frac{e \hat{V} \omega_{\mathrm{rf}}}{2 p_{0}} \delta_{L}\left(s-s_{\mathrm{rf}}\right)\left[\tau-\frac{\eta(s)}{\beta_{0} c} p_{x}+\frac{\xi(s)}{\beta_{0} c} x\right]^{2} .
\end{aligned}
$$

There should be no cross terms, such as the $x \delta$ and $p_{x} \delta$ terms. For now the $\tau$ cross terms are ignored. Thus, $\xi$ and $\eta$ must satisfy the relations

$$
\xi(s)=\frac{d \eta}{d s} \quad \frac{d^{2} \eta}{d s^{2}}+\left[h(s)^{2}-K(s)\right] \eta(s)=h(s) .
$$

The $\eta$ that solves these equations and is periodic in $s$ with period $L$ is known as the dispersion function $[19,68]$. There are still cross terms proportional to $\tau \delta$. These cross terms can be minimized by designing the lattice so that the dispersion function and its derivative are small at the rf cavities, which is usually done.

The quadratic part of the Hamiltonian thus becomes

$$
\begin{aligned}
\frac{1}{2} p_{x}^{2}+\frac{1}{2} p_{y}^{2}+\frac{1}{2}\left[h^{2}(s)-K(s)\right] x^{2} & +\frac{1}{2} K(s) y^{2} \\
& -\frac{1}{2} \frac{\delta^{2}}{\beta_{0}^{2} c^{2}}\left[h(s) \eta(s)-\frac{1}{\gamma_{0}^{2}}\right]-\frac{e \hat{V} \omega_{\mathrm{rf}}}{2 p_{0}} \delta_{L}\left(s-s_{\mathrm{rf}}\right) r^{2}
\end{aligned}
$$

where the dispersion function and its derivative have been taken to be zero at $s_{\text {rf }}$. The accuracy of this approximation can be determined by computing

$$
\xi_{\eta}=\frac{\sqrt{\epsilon_{x} J_{\eta}\left(s_{\mathrm{r}}\right)}}{\sigma_{\ell}}
$$

where

$$
J_{\eta}(s)=\left(\frac{d \eta}{d s}\right)^{2} \beta_{x}(s)+2 \eta(s) \frac{d \eta}{d s} \alpha_{x}(s)+\eta^{2}(s) \frac{1+\alpha_{x}^{2}(s)}{\beta_{x}(s)},
$$

and $\beta_{x}, \alpha_{x}$, and $\epsilon_{x}$ are the quantities defined in appendix A.3 for the horizontal plane, and $\sigma_{\ell}$ is the rms bunch half-length. As long as $\xi_{\eta}$ is small compared with any growth rates (in inverse turns) that are computed, neglecting the $\tau$ coupling terms at the rf cavities is a good approximation.

Under the transformations (3.49) and (3.50), the nonlinear Hamiltonian (3.47) becomes

$$
\begin{aligned}
& \frac{1}{2} p_{x}^{2}+\frac{1}{2} p_{y}^{2}+\frac{1}{2}\left[h^{2}(s)-K(s)\right] x^{2}+\frac{1}{2} K(s) y^{2}-\frac{1}{2} \frac{\delta^{2}}{\beta_{0}^{2} c^{2}}\left[h(s) \eta(s)-\frac{1}{\gamma_{0}^{2}}\right] \\
& -\frac{e \hat{V} \omega_{\mathrm{rf}}}{2 p_{0}} \delta_{L}\left(s-s_{\mathrm{rf}}\right) \tau^{2}+\frac{1}{2}\left[\left(p_{x}-\frac{\eta^{\prime}(s) \delta}{\beta_{0} c}\right)^{2}+p_{y}^{2}+\frac{\delta^{2}}{\gamma_{0}^{2} \beta_{0}^{2} c^{2}}\right]\left[h(s) x+(1-h(s) \eta(s)) \frac{\delta}{\beta_{0} c}\right] \\
& -\frac{S(s)}{6}\left[\left(x-\frac{\eta(s) \delta}{\beta_{0} c}\right)^{3}-3\left(x-\frac{\eta(s) \delta}{\beta_{0} c}\right) y^{2}\right]+\frac{e \hat{V} \omega_{\mathrm{rf}}}{24 p_{0}} \delta_{L}\left(s-s_{\mathrm{rf}}\right) \tau^{4} \\
& +\frac{e}{\beta_{0} p_{0} c}\left[1+h(s) x+\left(\frac{1}{\gamma_{0}^{2}}-h(s) \eta(s)\right) \frac{\delta}{\beta_{0} c}\right] f\left(x-\frac{\eta(s) \delta}{\beta_{0} c}, y, \tau+\frac{x \eta^{\prime}(s)-p_{x} \eta(s)}{\beta_{0} c}, s\right) \text {. }
\end{aligned}
$$

Note that the dispersion and its derivative are still taken to be zero at $s_{\mathrm{rf}}$.

\subsubsection{General Nonlinear Hamiltonian with Wakefields}

The next task is to take the Hamiltonian due to magnets and if cavities and convert it to action-angle variables. While this is in general not possible in the nonlinear case, the socalled normalization can be done as a perturbation series about the origin of phase space [23, 26,43]. See appendices A.3, A.4, A.5, and A.6 for some possible methods for accomplishing this. The term in the Hamiltonian due to magnets and rf cavities is then simply $H_{0}(J)$. Note that the discussion in this section is independent of what the Hamiltonian due to magnets and rf cavities looks like; it may be the first three lines of equation (3.57), but it need not be.

The normalization induces a symplectic transformation from action-angle variables to the previously described phase space coordinates of the ring (before the dispersion transformation (3.49)). The transformation can be partially described by the functions, $\hat{x}(J, \theta, s)$, $\hat{y}(J, \theta, s)$, and $\hat{\tau}(J, \theta, s)+\hat{\tau}_{r 0}$, along with similar functions for the other phase space variables. The transformation described is for particles in the rth rf bucket; because all the 
buckets are identical, the function $\hat{\tau}(J, \theta, s)$ is independent of the bucket that the transformation is being done for.

Now the wakefield terms as described in section 3.1 .3 can be added to $H_{0}(J)$. When doing so, one must take into account the fact that when a bunch passes by a position $s$, it sees the wakefields it deposited there on all previous turns. There are assumed to be $N_{r}$ particles with charge $e$ in the rf bucket that is centered around $\hat{\tau}_{r 0}$. Thus, the Hamiltonian for particles in the $r$ th bucket is

$$
\begin{array}{r}
H_{r}=H_{0}(J)+\frac{r_{0}}{\beta_{0}^{2} \gamma_{0}} \sum_{k n} N_{n}\{ \\
\quad \int W_{0}\left(\hat{\tau}(\theta, J, s)-\hat{\tau}\left(\theta^{\prime}, J^{\prime}, s\right)+\hat{\tau}_{r 0}-\hat{\tau}_{n 0}+k T_{0}, s\right) \hat{\Psi}_{n}\left(\theta^{\prime}, J^{\prime}, s-k L\right) d^{3} \theta^{\prime} d^{3} J^{\prime} \\
+\hat{x}(\theta, J, s) \int \hat{x}\left(\theta^{\prime}, J^{\prime}, s\right) W_{\perp}\left(\hat{\tau}(\theta, J, s)-\hat{\tau}\left(\theta^{\prime}, J^{\prime}, s\right)+\hat{\tau}_{r 0}-\hat{\tau}_{n 0}+k T_{0}, s\right) \\
\hat{\Psi}_{n}\left(\theta^{\prime}, J^{\prime}, s-k L\right) d^{3} \theta^{\prime} d^{3} J^{\prime} \\
+\hat{y}(\theta, J, s) \int \hat{y}\left(\theta^{\prime}, J^{\prime}, s\right) W_{\perp}\left(\hat{\tau}(\theta, J, s)-\hat{\tau}\left(\theta^{\prime}, J^{\prime}, s\right)+\hat{\tau}_{r 0}-\hat{\tau}_{n 0}+k T_{0}, s\right) \\
\left.\hat{\Psi}_{n}\left(\theta^{\prime}, J^{\prime}, s-k L\right) d^{3} \theta^{\prime} d^{3} J^{\prime}\right\}
\end{array}
$$

where $\hat{\Psi}_{n}$ is the phase space distribution function for the particles in the $n$th rf bucket, $r_{0}$ is the classical radius of the electron, and $T_{0}=L / \beta_{0} c$. The wakefield terms have been simplified to include only the two lowest multipoles of the wakefield.

The $\delta$ and $x$ terms in the factor multiplying $f$ in equation (3.57) have also been ignored in equation (3.58). If the $\delta$ term had been included, it would have introduced corrections of order $2 \pi \nu_{s} \sigma_{t} / L$, where $\nu_{s}$ is the synchrotron tune. This should be an extremely small quantity for any real ring (for example, it is about $10^{-6}$ for the PEP-II low energy ring [49]). Similarly, if the $x$ term had been included, it would have introduced corrections of order $2 \pi \sqrt{\beta_{x} \epsilon_{x}} / L_{;}$this also should be a very small correction (about $3 \times 10^{-6}$ for the PEP-II low energy ring [49]).

As will be described in section 3.3, there should be a set of distributions $\Psi_{r 0}$ which satisfy the Vlasov equation for the corresponding $H_{r}$ with $\hat{\Psi}_{n}$ replaced by $\Psi_{n 0}$, and are independent of $s$. The goal of this analysis is to perturb $\Psi_{n 0}$ and determine whether that perturbation is stable. Thus, it is convenient to write the Hamiltonian in terms of the perturbation to $\hat{\Psi}_{n}$.
The first step is to change variables to a second set of action-angle variables. These are the action-angle variables which make the Hamiltonian (3.58) a function of the action variables only, when $\dot{\hat{\Psi}}_{n}$ is replaced by $\Psi_{n 0}$. As will become clear in section $2.3, \Psi_{n 0}$ will only be a function of the action in these variables. Since each bunch has a different number of particles in general, the resulting transformations will depend on which $\mathrm{rf}$ bucket is begin considered. The transformation functions for particles in the $r$ th bucket are written as $x_{r}(\theta, J, s)+x_{r 0}(s), y_{r}(\theta, J, s)+y_{r 0}(s)$, and $\tau_{r}(\theta, J, s)+\tau_{r 0}(s)$. The offset $\tau_{r 0}(s)$ is chosen so that $\tau_{r}(\theta, 0, s)=0 ; x_{r 0}(s)$ and $y_{r 0}(s)$ are chosen the same way. These offsets may be absorbed into the functions $\tau_{r}, x_{r}$ and $y_{r}$ if desired. The functions $x_{r 0}(s)$ and $y_{r 0}(s)$ describe how the closed orbit that the particles are trying to follow differs from the center of the beam pipe. In terms of these action-angle variables, $\hat{\Psi}_{r}=\Psi_{\mathrm{r} 0}(J)+\Psi_{r}(\theta, J, s)$, where $\Psi_{r}$ is a perturbation to $\Psi_{r 0}$. Thus, the Hamiltonian for particles in the $r$ th bucket is thus

$$
\begin{aligned}
& H_{r}=H_{r 0}(J)+\frac{r_{0}}{\beta_{0}^{2} \gamma_{0}} \sum_{k n} N_{n}\{ \\
& \int W_{0}\left(\tau_{r}(\theta, J, s)-\tau_{n}\left(\theta^{\prime}, J^{\prime}, s\right)+\tau_{r 0}(s)-\tau_{n 0}(s)+k T_{0}, s\right) \Psi_{n}\left(\theta^{\prime}, J^{\prime}, s-k L\right) d^{3} \theta^{\prime} d^{3} J^{\prime} \\
& +\left[x_{r}(\theta, J, s)+x_{r 0}(s)\right] \int\left[x_{n}\left(\theta^{\prime}, J^{\prime}, s\right)+x_{n 0}(s)\right] \\
& \quad W_{\perp}\left(\tau_{r}(\theta, J, s)-\tau_{n}\left(\theta^{\prime}, J^{\prime}, s\right)+\tau_{r 0}(s)-\tau_{n 0}(s)+k T_{0}, s\right) \Psi_{n}\left(\theta^{\prime}, J^{\prime}, s-k L\right) d^{3} \theta^{\prime} d^{3} J^{\prime} \\
& +\left[y_{r}(\theta, J, s)+y_{r 0}(s)\right] \int\left[y_{n}\left(\theta^{\prime}, J^{\prime}, s\right)+y_{n 0}(s)\right] \\
& \left.\quad W_{\perp}\left(\tau_{r}(\theta, J, s)-\tau_{n}\left(\theta^{\prime}, J^{\prime}, s\right)+\tau_{r 0}(s)-\tau_{n 0}(s)+k T_{0}, s\right) \Psi_{n}\left(\theta^{\prime}, J^{\prime}, s-k L\right) d^{3} \theta^{\prime} d^{3} J^{\prime}\right\}
\end{aligned}
$$

The combining of the terms due to the unperturbed distribution into $H_{r 0}$ is referred to as "potential-well distortion" [11,46-48], because its effect is as if the potential well keeping the particles focussed were being changed by an external force. No attempt will be made here to quantitatively compute this effect. Some commentary will be made later on the possible effects of potential-well distortion.

\subsubsection{Feedback}

Feedback (see section 2.1.3) can be added to the system by modifying the Hamiltonian (3.58). As described in section 2.1.3, feedback will give a force on a particle at a position $s$ 
which is related to the position of the particle at a position $s-\Delta s$. From the arguments in section 3.1, it follows that if only time-independent components (i.e., components which have no sense of "wall-clock time") transmit the signal from the pickup to the kicker, then the force can be described through a wakefield-like term, similar to equation (3.25).

By this argument, the feedback system simply adds terms to the Hamiltonian (3.58) of

$$
\begin{aligned}
& \frac{r_{0}}{\beta_{0}^{2} \gamma_{0}} \sum_{k n} N_{n}\{ \\
& \int W_{0}^{\mathrm{FB}}\left(\hat{\tau}(\theta, J, s)-\hat{\tau}\left(\theta^{\prime}, J^{\prime}, s-\Delta s\right)+\hat{\tau}_{r 0}-\hat{\tau}_{n 0}+\Delta s / \beta_{0} c+k T_{0}, s\right) \\
& \hat{\Psi}_{n}\left(\theta^{\prime}, J^{\prime}, s-\Delta s-k L\right) d^{3} \theta^{\prime} d^{3} J^{\prime} \\
& +\hat{x}(\theta, J, s) \int W_{x}^{\mathrm{FB}}\left(\hat{\tau}(\theta, J, s)-\hat{\tau}\left(\theta^{\prime}, J^{\prime}, s-\Delta s\right)+\hat{\tau}_{r 0}-\hat{\tau}_{n 0}+\Delta s / \beta_{0} c+k T_{0}, s\right) \\
& \hat{x}\left(\theta^{\prime}, J^{\prime}, s-\Delta s\right) \hat{\Psi}_{n}\left(\theta^{\prime}, J^{\prime}, s-\Delta s-k L\right) d^{3} \theta^{\prime} d^{3} J^{\prime} \\
& +\hat{y}(\theta, J, s) \int W_{y}^{\mathrm{FB}}\left(\hat{\tau}(\theta, J, s)-\hat{\tau}\left(\theta^{\prime}, J^{\prime}, s-\Delta s\right)+\hat{\tau}_{r 0}-\hat{\tau}_{n 0}+\Delta s / \beta_{0} c+k T_{0}, s\right) \\
& \left.\hat{y}\left(\theta^{\prime}, J^{\prime}, s-\Delta s\right) \hat{\Psi}_{n}\left(\theta^{\prime}, J^{\prime}, s-k L-\Delta s\right) d^{3} \theta^{\prime} d^{3} J^{\prime}\right\}
\end{aligned}
$$

The arguments of the $W^{\mathrm{FB}}$ have been chosen such that the condition for causality is precisely that $W^{\mathrm{FB}}(\tau)$ is zero for $\tau<0$. Several sets of similar terms could be added, all with different $\Delta s$.

Instead of a subscript $\perp$, the transverse feedback wakes have a subscript of $x$ or $y$. This is because the horizontal and vertical transverse feedback wakes must be different, since the horizontal and vertical tunes are different (the tune determines the optimal $\Delta s$, actually). This implies a breaking of the cylindrical symmetry that allowed the wake potential to be written in the form (3.25) in the first place. If the weaker assumption of horizontal and vertical reflection symmetry is made, then an $m=1$ source distribution ( $m$ in this case refers to the azimuthal charge distribution in real space, not the longitudinal phase space charge distribution) will not cause any $m=0$ forces (or $m=2$ forces, etc.). Neither will vertical oscillations drive horizontal oscillations. Thus, the expansion in equation (3.60) gives a good approximation even without cylindrical symmetry.

Note that just as the wakefields have a Fourier transform known as the impedance (see 
section 3.1.4), these feedback wakes also have a Fourier transform, which will be defined as

$$
\begin{gathered}
Z_{x}^{\mathrm{FB}}(\omega, s)=i \int_{-\infty}^{\infty} W_{x}^{\mathrm{FB}}(\tau, s) e^{i \omega \tau} d \tau \\
Z_{y}^{\mathrm{FB}}(\omega, s)=i \int_{-\infty}^{\infty} W_{y}^{\mathrm{FB}}(\tau, s) e^{i \omega \tau} d \tau \\
Z_{\|}^{\mathrm{FB}}(\omega, s)=-\frac{1}{\beta_{0} c} \int_{-\infty}^{\infty}\left(W_{0}^{\mathrm{FB}}\right)^{\prime}(\tau, s) e^{i \omega \tau} d \tau .
\end{gathered}
$$

A similar impedance-like quantity for feedback was defined by Wang [18].

Once potential-well distortion has been taken into account (now including the feedback), then the Hamiltonian becomes the Hamiltonian (3.59) plus the terms

$$
\begin{array}{r}
\frac{r_{0}}{\beta_{0}^{2} \gamma_{0}} \sum_{k n} N_{n}\{ \\
\quad \int W_{0}^{\mathrm{FB}}\left(\tau_{r}(\theta, J, s)-\tau_{n}\left(\theta^{\prime}, J^{\prime}, s-\Delta s\right)+\tau_{r 0}(s)-\tau_{n 0}(s-\Delta s)+\Delta s / \beta_{0} c+k T_{0}, s\right) \\
\Psi_{n}\left(\theta^{\prime}, J^{\prime}, s-\Delta s-k L\right) d^{3} \theta^{\prime} d^{3} J^{\prime} \\
+\left[x_{r}(\theta, J, s)+x_{r 0}(s)\right] \int\left[x_{n}\left(\theta^{\prime}, J^{\prime}, s-\Delta s\right)+x_{n 0}(s-\Delta s)\right] \Psi_{n}\left(\theta^{\prime}, J^{\prime}, s-\Delta s-k L\right) \\
W_{x}^{\mathrm{FB}}\left(\tau_{r}(\theta, J, s)-\tau_{n}\left(\theta^{\prime}, J^{\prime}, s-\Delta s\right)+\tau_{r 0}(s)-\tau_{n 0}(s-\Delta s)+\Delta s / \beta_{0} c+k T_{0}, s\right) d^{3} \theta^{\prime} d^{3} J^{\prime} \\
+\left[y_{r}(\theta, J, s)+y_{r 0}(s)\right] \int\left[y_{n}\left(\theta^{\prime}, J^{\prime}, s-\Delta s\right)+y_{n 0}(s-\Delta s)\right] \Psi_{n}\left(\theta^{\prime}, J^{\prime}, s-\Delta s-k L\right) \\
\left.W_{y}^{\mathrm{FB}}\left(\tau_{r}(\theta, J, s)-\tau_{n}\left(\theta^{\prime}, J^{\prime}, s-\Delta s\right)+\tau_{r 0}(s)-\tau_{n 0}(s-\Delta s)+\Delta s / \beta_{0} c+k T_{0}, s\right) d^{3} \theta^{\prime} d^{3} J^{\prime}\right\} .
\end{array}
$$

\subsection{Vlasov Equation}

For non-interacting particles whose motion is governed by a Hamiltonian, Liouville's theorem states that the s-evolution of the probability distribution of particles is governed by the Vlasov equation $[11,41,43]$

$$
\frac{\partial \Psi}{\partial s}+[\Psi, H]=0
$$

The [] are Poisson brackets $[3,41,43]$. If the particles do interact, as in our system, the evolution of the distribution function is governed instead by an infinite, coupled series of equations known as the BBGKY hierarchy $[2,20,36]$. That hierarchy is generally truncated at some point; if it is truncated such that the two-particle distribution function $\Psi\left(z_{1}, z_{2}, t\right)$ is equal to the product of the one-particle distribution functions $\Psi\left(z_{1}, t\right) \Psi\left(z_{2}, t\right)$, then equation (3.65) still governs the evolution of the distribution function $\Psi$. In other words, the so-called two-particle correlation function is zero-the distribution for two particles is completely determined by the distribution for a single particle. This is a common approximation which is beyond the scope of this dissertation to analyze.

The phase space distribution $\Psi$ is written as a sum over the phase space distributions in each bucket $\hat{\Psi}_{n}$ :

$$
\Psi(z, s)=\frac{\sum_{r=0}^{h_{\mathrm{r} f}-1} N_{n} \hat{\Psi}_{n}(z, s)}{\sum_{r=0}^{h_{\mathrm{r} f}-1} N_{n}} .
$$

If each bunch distribution $\hat{\Psi}_{n}$ is zero outside of an interval $I_{n}$ in $\tau$, and no two of the $I_{n}$ intersect, then the Vlasov equation (3.65) can be written for each bunch distribution:

$$
\frac{\partial \hat{\Psi}_{r}}{\partial t}+\left[\hat{\Psi}_{r}, H_{r}\right]=0
$$

The $H_{r}$ here is the Hamiltonian for bunches in the $r$ th bucket given by equation (3.58) or equation (3.60). This Hamiltonian causes equation (3.67) to really be a coupled set of partial integro-differential equations which are nonlinear in $\hat{\Psi}_{r}$ : the $\hat{\Psi}_{\tau}$ are coupled to all the other $\hat{\Psi}_{n}$ through $H_{r}$.

As indicated in section 3.2.5, the first step is to find a $s$-independent solution of the coupled equations (3.67). The details of finding such a solution are beyond the scope of this dissertation; see $[11,46-48]$. Note that in the transverse case which is analyzed in chapter 5 , this step is unnecessary. This is because any solution which is only a function of the action variables will have no transverse moment, and thus gives a self-consistent solution by default. Once a self-consistent solution is found, one determines whether or not a small perturbation to such a solution is stable. As described in section 3.2.5 or section 3.2.6, the distribution $\hat{\Psi}_{r}$ is written as $\Psi_{r 0}+\Psi_{r}$. Since $\Psi_{r 0}$ is independent of time, after the final change of variables described in section 3.2.5 (after which the Hamiltonian with the perturbation $\Psi_{r}=0$ depends only on $J$ ), the Vlasov equation (3.67) shows that $\Psi_{r 0}$ can only depend on $J$. Writing the $H_{r}$ of equation (3.59) or equation (3.64) as $H_{0 r}+\Delta H_{r}$, the 
Vlasov equation becomes (ignoring second order terms in $\Psi_{r}$ )

$$
\frac{\partial \Psi_{r}}{\partial s}+\frac{\omega_{r}(J)}{\beta_{0} c} \cdot \frac{\partial \Psi_{r}}{\partial \theta}-\frac{\partial \Psi_{r 0}}{\partial J} \cdot \frac{\partial \Delta H_{r}}{\partial \theta}=0
$$

where

$$
\omega_{r}(J)=\beta_{0} c \frac{\partial H_{0 r}}{\partial J}
$$

\section{Chapter 4}

\section{Eigenvalue System}

To determine the stability of the beam, one needs to find all solutions of (3.68), and determine if any of those solutions are unstable. Solutions will be considered unstable if they grow exponentially, and stable otherwise. Begin by defining the Fourier transform of $\Psi_{r}$ :

$$
\Psi_{r}(\theta, J, \Omega)=\frac{1}{L} \int \Psi_{r}(\theta, J, s) e^{i \Omega s / \beta_{0} c} d s
$$

Then equation (3.68) becomes an equation for the $\Psi_{\tau}(\theta, J, \Omega)$. If there is a solution for which $\Psi_{r}(\theta, J, \Omega)$ is nonzero and $\Im\{\Omega\}>0$, the system is unstable, since such a solution would have exponentially growing parts from equation (4.1). In practice, there are damping mechanisms which are not taken into account in the theory; in such cases, the condition for instability changes to $\Im\{\Omega\}>2 \pi / \tau_{d}$, where $\tau_{d}$ is the damping time due to these other damping mechanisms.

Using the definition of $\Psi_{r}(\theta, J, \Omega)$, as well as the definition of impedance given in equations (3.26) and (3.27), the Hamiltonian (3.59) becomes

$$
\begin{gathered}
H_{r}=H_{r 0}(J)-\frac{i r_{0}}{2 \pi \beta_{0}^{2} \gamma_{0}} \sum_{n p} N_{n} \int\left(\beta_{0} c \frac{Z_{\|}\left(p \omega_{0}+\Omega^{\prime}, s\right)}{p \omega_{0}+\Omega^{\prime}}+Z_{\perp}\left(p \omega_{0}+\Omega^{\prime}, s\right)\{\right. \\
\left.\left.\left[x_{r}(\theta, J, s)+x_{r 0}(s)\right]\left[x_{n}\left(\theta^{\prime}, J^{\prime}, s\right)+x_{n 0}(s)\right]+\left[y_{r}(\theta, J, s)+y_{r 0}(s)\right]\left[y_{n}\left(\theta^{\prime}, J^{\prime}, s\right)+y_{n 0}(s)\right]\right\}\right) \\
e^{-i\left(p \omega_{0}+\Omega^{\prime}\right) \tau_{r}(\theta, J, s)} e^{i\left(p \omega_{0}+\Omega^{\prime}\right) \tau_{n}\left(\theta^{\prime}, J^{\prime}, s\right)} e^{-i\left(p \omega_{0}+\Omega^{\prime}\right) \tau_{r 0}(s)} e^{i\left(p \omega_{0}+\Omega^{\prime}\right) \tau_{n 0}(s)} \\
e^{-i \Omega^{\prime} s / \beta_{0} c_{1}} \Psi_{n}\left(\theta^{\prime}, J^{\prime}, \Omega^{\prime}\right) d^{3} \theta^{\prime} d^{3} J^{\prime} d \Omega^{\prime}
\end{gathered}
$$

where $\omega_{0}=2 \pi / T_{0}$ is the angular revolution frequency of a particle following the ideal orbit $C$. One could write $\Psi_{n}$ over a set of basis functions in $\theta$ and $J$, which would turn the 
Vlasov equation in to a set of algebraic equations. However, it is simpler to work in terms of the quantities after they have been integrated in $\theta$ and $J$ as in (4.2).

It is first of all useful to define the functions

$$
\begin{aligned}
f_{n}^{\alpha}(\theta, J, s) & = \begin{cases}1 & \alpha=0 \\
x_{n}(\theta, J, s) & \alpha=x \\
y_{n}(\theta, J, s) & \alpha=y\end{cases} \\
f_{n 0}^{\alpha}(s) & = \begin{cases}0 & \alpha=0 \\
x_{n 0}(s) & \alpha=x \\
y_{n 0}(s) & \alpha=y\end{cases} \\
Z_{\alpha}(\omega, s) & = \begin{cases}\beta_{0} c \frac{Z_{\|}(\omega, s)}{\omega} & \alpha=0 \\
Z_{\perp}(\omega, s) & \alpha=x, y .\end{cases}
\end{aligned}
$$

Next, define the quantities

$$
D_{n p}^{\alpha}(\Omega, s)=\int f_{n}^{\alpha}(\theta, J, s) e^{i\left(p \omega_{0}+\Omega\right) \tau_{n}(\theta, J, s)} \Psi_{n}(\theta, J, \Omega) d^{3} \theta d^{3} J .
$$

The $D_{n p}^{\alpha}$ are just the lowest multipole (monopole and two dipole) moments of the perturbation to the distribution, Fourier analyzed. The frequency $\Omega$ corresponds to the frequency of oscillations in the bunch. The bunch passes by a given point in the lab every $T_{0}$; thus, in the lab frame, the bunch perturbation should have a frequency spectrum with frequency components $p \omega_{0}+\Omega$ for every integer $p$. Thus, $D_{n p}^{\alpha}(\Omega, s)$ gives the frequency component of the perturbation to bunch $n$ at the frequency $p \omega_{0}+\Omega$ in the lab frame. The Hamiltonian can now be written as

$$
\begin{aligned}
& H_{r}=H_{r 0}(J)-\frac{i r_{0}}{2 \pi \beta_{0}^{2} \gamma_{0}} \sum_{\alpha n p} N_{n} \int Z_{\alpha}\left(p \omega_{0}+\Omega^{\prime}, s\right) \\
& {\left[f_{r}^{\alpha}(\theta, J, s)+f_{r 0}^{\alpha}(s)\right]\left[D_{n p}^{\alpha}\left(\Omega^{\prime}, s\right)+f_{n 0}^{\alpha}(s) D_{n p}^{0}\left(\Omega^{\prime}, s\right)\right]} \\
& e^{-i\left(p \omega_{0}+\Omega^{\prime}\right) \tau_{r}(\theta, J, s)} e^{-i\left(p \omega_{0}+\Omega^{\prime}\right) \tau_{\mathrm{r}}(s)} e^{i\left(p \omega_{0}+\Omega^{\prime}\right) \tau_{\mathrm{n} 0}(s)} e^{-i \Omega^{\prime} s / \beta_{0} c} d \Omega^{\prime}
\end{aligned}
$$

The Vlasov equation (3.68) can now be written for $\Psi_{r}(\theta, J, \Omega)$ using the Hamiltonian
(4.7):

$$
\left[-i \Omega+\omega_{r}(J) \cdot \frac{\partial}{\partial \theta}\right] \Psi_{r}(\theta, J, \Omega)=-\frac{i r_{0} c^{2}}{\gamma_{0} L^{2}} \sum_{\alpha k n p} N_{n} \frac{\partial \Psi_{r 0}}{\partial J} \cdot \frac{\partial}{\partial \theta} \int_{0}^{L} d s^{\prime} e^{-2 \pi i k s^{\prime} / L}
$$

$Z_{\alpha}\left(p \omega_{0}+\Omega, s^{\prime}\right)\left[f_{r}^{\alpha}\left(\theta, J, s^{\prime}\right)+f_{r 0}^{\alpha}\left(s^{\prime}\right)\right]\left[D_{n, p-k}^{\alpha}\left(\Omega+k \omega_{0}, s^{\prime}\right)+f_{n 0}^{\alpha}\left(s^{\prime}\right) D_{n, p-k}^{0}\left(\Omega+k \omega_{0}, s^{\prime}\right)\right]$

$$
e^{-i\left(p \omega_{0}+\Omega\right) \tau_{r}\left(\theta, J, s^{\prime}\right)} e^{-i\left(p \omega_{0}+\Omega\right) \tau_{r}\left(s^{\prime}\right)} e^{i\left(p \omega_{0}+\Omega\right) \tau_{n 0}\left(s^{\prime}\right)} \text {. (4.8) }
$$

Define the operator

$$
G(\Omega, \omega)=-i \Omega+\omega \cdot \frac{\partial}{\partial \theta}
$$

Applying the inverse of this operator to equation (4.8) produces an equation for $\Psi_{r}$. Equation (4.6) can then be used to turn $\Psi_{r}$ into $D_{r q}^{\beta}$, giving the system of equations

$$
\begin{aligned}
& D_{r q}^{\beta}(\Omega, s)=-\frac{i r_{0} c^{2}}{\gamma_{0} L^{2}} \sum_{\alpha k n p} N_{n} \int_{0}^{L} d s^{\prime} e^{-2 \pi i k s^{\prime} / L} e^{-i\left(p \omega_{0}+\Omega\right)\left(\tau_{r 0}\left(s^{\prime}\right)-\tau_{n} 0\left(s^{\prime}\right)\right]} Z_{\alpha}\left(p \omega_{0}+\Omega, s^{\prime}\right) \\
& {\left[R_{q p r}^{\beta \alpha}\left(\Omega, s, s^{\prime}\right)+f_{r 0}^{\alpha}\left(s^{\prime}\right) R_{q p r}^{\beta 0}\left(\Omega, s, s^{\prime}\right)\right]\left[D_{n, p-k}^{\alpha}\left(\Omega+k \omega_{0}, s^{\prime}\right)+f_{n 0}^{\alpha}\left(s^{\prime}\right) D_{n, p-k}^{0}\left(\Omega+k \omega_{0}, s^{\prime}\right)\right],}
\end{aligned}
$$

where

$$
\begin{aligned}
R_{q p r}^{\beta \alpha}\left(\Omega, s, s^{\prime}\right) & =\int f_{r}^{\beta}(\theta, J, s) e^{i\left(q \omega_{0}+\Omega\right) \tau_{r}(\theta, J, s)} \\
G^{-1}\left(\Omega, \omega_{r}(J)\right) & \left\{\frac{\partial \Psi_{r 0}}{\partial J} \cdot \frac{\partial}{\partial \theta}\left[f_{r}^{\alpha}\left(\theta, J, s^{\prime}\right) e^{-i\left(p \omega_{0}+\Omega\right) \tau_{r}\left(\theta, J, s^{\prime}\right)}\right]\right\} d^{3} \theta d^{3} J .
\end{aligned}
$$

By taking the complex conjugate of (4.10) and redefining the $D$ 's slightly, it is straightforward to show that if there is a solution of (4.10) with a frequency $\Omega$, there is also a solution with a frequency $-\Omega^{*}$. Thus, every solution has a positive and negative frequency solution which have the same imaginary parts.

Write each of the $R^{\alpha \beta}$ in the form

$$
R_{q p r}^{\beta \alpha}\left(\Omega, s, s^{\prime}\right)=\sum_{a} B_{q r a}^{\beta \alpha}(\Omega, s) C_{p r a}^{\beta \alpha}\left(\Omega, s^{\prime}\right) .
$$

At the very least, this can be achieved by taking the Taylor series of $(4.12)$ in $\left(q \omega_{0}+\Omega\right)$ and $\left(p \omega_{0}+\Omega\right)$, then Fourier analyzing in $s$ or $s^{\prime}$. The expansion (4.12) enables a transformation to the basis functions $D_{r a}^{\beta \alpha}(\Omega)$ and $M_{r a}^{\beta \alpha}(\Omega)$ :

$$
\begin{aligned}
D_{r a}^{\beta \alpha}(\Omega)=\sum_{k n p} N_{n} \int_{0}^{L} d s^{\prime} e^{-2 \pi i k s^{\prime} / L} e^{-i\left(p \omega_{0}+\Omega\right)\left[r_{r 0}\left(s^{\prime}\right)-\tau_{n} 0\left(s^{\prime}\right)\right]} Z_{\alpha}\left(p \omega_{0}+\Omega, s^{\prime}\right) \\
\\
C_{p r a}^{\beta \alpha}\left(\Omega, s^{\prime}\right)\left[D_{n, p-k}^{\alpha}\left(\Omega+k \omega_{0}, s^{\prime}\right)+f_{n 0}^{\alpha}\left(s^{\prime}\right) D_{n, p-k}^{0}\left(\Omega+k \omega_{0}, s^{\prime}\right)\right]
\end{aligned}
$$




$$
\begin{aligned}
M_{r a}^{\beta \alpha}(\Omega)=\sum_{k n p} N_{n} \int_{0}^{L} d s^{\prime} f_{\tau 0}^{\alpha}\left(s^{\prime}\right) e^{-2 \pi i k s^{\prime} / L} e^{-i\left(p \omega_{0}+\Omega\right)\left[\tau_{0}\left(s^{\prime}\right)-\tau_{n 0}\left(s^{\prime}\right)\right]} Z_{\alpha}\left(p \omega_{0}+\Omega, s^{\prime}\right) \\
\\
C_{p r a}^{\beta 0}\left(\Omega, s^{\prime}\right)\left[D_{n, p-k}^{\alpha}\left(\Omega+k \omega_{0}, s^{\prime}\right)+f_{n 0}^{\alpha}\left(s^{\prime}\right) D_{n, p-k}^{0}\left(\Omega+k \omega_{0}, s^{\prime}\right)\right] .
\end{aligned}
$$

Note that $M_{r a}^{\beta \alpha}$ only exists for $\alpha \neq 0$. Also, $M_{r a}^{\beta \alpha}$ is identical to $D_{r a}^{\beta 0}$ if $f_{r 0}^{\alpha}$ is a constant function (including zero). Equation (4.12) and the transformations (4.13) and (4.14) turn equation (4.10) into

$$
\begin{aligned}
& D_{n b}^{\gamma \beta}(\Omega)=-\frac{i r_{0} c^{2}}{\gamma_{0} L^{2}} \sum_{\alpha a k r q} N_{r} \int_{0}^{L} d s e^{-2 \pi i k s / L} e^{-i\left(q \omega_{0}+\Omega\right)\left[\tau_{n 0}(s)-\tau_{r} 0(s)\right]} Z_{\beta}\left(q \omega_{0}+\Omega, s\right) \\
& C_{q n b}^{\gamma \beta}(\Omega, s)\left[B_{q-k, r, a}^{\beta \alpha}\left(\Omega+k \omega_{0}, s\right) D_{r a}^{\beta \alpha}\left(\Omega+k \omega_{0}\right)+B_{q-k, r, a}^{\beta 0}\left(\Omega+k \omega_{0}, s\right) M_{r a}^{\beta \alpha}\left(\Omega+k \omega_{0}\right)\right. \\
& \left.+f_{r 0}^{\beta}(s) B_{q-k, r, a}^{0 \alpha}\left(\Omega+k \omega_{0}, s\right) D_{r a}^{0 \alpha}\left(\Omega+k \omega_{0}\right)+f_{r 0}^{\beta}(s) B_{q-k, r, a}^{00}\left(\Omega+k \omega_{0}, s\right) M_{r a}^{0 \alpha}\left(\Omega+k \omega_{0}\right)\right] \\
& M_{n b}^{\gamma \beta}(\Omega)=-\frac{i r_{0} c^{2}}{\gamma_{0} L^{2}} \sum_{\alpha a k r q} N_{r} \int_{0}^{L} d s e^{-2 \pi i k s / L} e^{-i\left(q \omega_{0}+\Omega\right)\left(\tau_{n 0}(s)-\tau_{r 0}(s)\right]} Z_{\beta}\left(\Omega+q \omega_{0}, s\right) f_{r 0}^{\beta}(s) \\
& C_{q n b}^{\gamma 0}(\Omega, s)\left[B_{q-k, r, a}^{\beta \alpha}\left(\Omega+k \omega_{0}, s\right) D_{r a}^{\beta \alpha}\left(\Omega+k \omega_{0}\right)+B_{q-k, r, a}^{\beta 0}\left(\Omega+k \omega_{0}, s\right) M_{r a}^{\beta \alpha}\left(\Omega+k \omega_{0}\right)\right. \\
& \left.+f_{r 0}^{\beta}(s) B_{q-k, r, a}^{0 \alpha}\left(\Omega+k \omega_{0}, s\right) D_{r a}^{0 \alpha}\left(\Omega+k \omega_{0}\right)+f_{r 0}^{\beta}(s) B_{q-k, r, a}^{00}\left(\Omega+k \omega_{0}, s\right) M_{r a}^{0 \alpha}\left(\Omega+k \omega_{0}\right)\right] .
\end{aligned}
$$

\subsection{Inversion of $G$}

Consider the operator $G$ defined in equation (4.9). The inverse of that operator is determined by solving the equation $G(\Omega, \omega) \Psi(\theta)=f(\theta)$. Both $\Psi$ and $f$ should be periodic functions of $\theta$, and so this must be taken into account when solving the equation.

There are (at least) two possible ways of doing this: one is to Fourier analyze in all components of $\theta$; the other is to Fourier analyze in all but one of the components, and then directly solve the remaining ordinary differential equation.

\subsubsection{Fourier Analyze in All Components .}

This method is very straightforward; if $\Psi_{m}$ and $f_{m}$ are the Fourier components of $\Psi$ and $f$ respectively, then

$$
\Psi_{m}=\frac{i f_{m}}{\Omega-m \cdot \omega}
$$

and thus

$$
\Psi(\theta)=\frac{i}{(2 \pi)^{d}} \sum_{m} \int d \theta^{\prime} \frac{f\left(\theta^{\prime}\right) e^{i m \cdot\left(\theta-\theta^{\prime}\right)}}{\Omega-m \cdot \omega},
$$

where $d$ is the number of dimensions of the vector $\theta$.

\subsubsection{Fourier Analyze in All but One Component}

A more useful form of the solution is often obtained by Fourier analyzing in all but one of the components of $\theta$. First of all, split all vectors into two components; one component has only one element. Without loss of generality, that component can be chosen to be the first. Thus, $\theta=\left(\theta_{0}, \bar{\theta}\right)$ and $\omega=\left(\omega_{0}, \bar{\omega}\right)$.

Next, Fourier analyze in $\bar{\theta}$. The resulting equation is

$$
-i(\Omega-\bar{m} \cdot \bar{\omega}) \Psi_{\bar{m}}\left(\theta_{0}\right)+\omega_{0} \frac{d \Psi_{\bar{m}}}{d \theta_{0}}=f_{\bar{m}}\left(\theta_{0}\right) .
$$

This ordinary differential equation can be solved assuming the boundary condition that $\Psi_{\tilde{m}}$ is $2 \pi$-periodic:

$$
\Psi_{\tilde{m}}\left(\theta_{0}\right)=\frac{i}{2 \omega_{0}} \csc \pi \frac{\Omega-\bar{m} \cdot \bar{\omega}}{\omega_{0}} \int_{-\pi}^{\pi} f_{\bar{m}}\left(\theta_{0}^{\prime}+\theta_{0}+\pi\right) e^{-i(\Omega-\bar{m} \cdot \bar{\omega}) \theta_{0} / \omega_{0}} d \theta_{0}^{\prime} .
$$

The full solution for $\Psi(\theta)$ follows immediately.

\subsection{No Potential-Well Distortion}

If potential-well distortion effects are ignored, then the functions $x_{r}, y_{r}$, and $\tau_{r}$ will be identical to the functions $\hat{x}, \hat{y}$, and $\hat{\tau}$ respectively (see subsection 3.2 .5 ). Thus, these functions no longer depend on $r$. Also, $\tau_{r 0}=\hat{\tau}_{r 0}$, while $x_{r 0}$ and $y_{r 0}$ are both zero.

These results vastly simplify the eigenvalue system. It is clear from the definition of $M_{\mathrm{ra}}^{\beta \alpha}(\Omega)$ given in equation (4.14) that the $M_{\mathrm{ra}}^{\beta \alpha}(\Omega)$ are zero. Thus, the eigenvalue system described by equations (4.15) and (4.16) simplifies to

$$
D_{n b}^{\gamma \beta}(\Omega)=-\frac{i r_{0} c^{2}}{\gamma_{0} L^{2}} \sum_{\alpha a k r q} N_{r} e^{-i\left(q \omega_{0}+\Omega\right)(n-r) T_{0} / h_{\mathrm{rf}}} Z_{q k b a n r}^{\gamma \beta \alpha}(\Omega) D_{r a}^{\beta \alpha}\left(\Omega+k \omega_{0}\right),
$$

where

$$
Z_{q k b a n r}^{\gamma \beta \alpha}(\Omega)=\int_{0}^{L} d s e^{-2 \pi i k s / L} Z_{\beta}\left(q \omega_{0}+\Omega, s\right) C_{q n b}^{\gamma \beta}(\Omega, s) B_{q-k, r, \mathrm{~s}}^{\beta \alpha}\left(\Omega+k \omega_{0}, s\right) .
$$


Note that $Z_{q k b a n r}^{\gamma \beta a}(\Omega)$ differs from $Z_{\beta}(\Omega)$; the former is a linear functional of the latter.

In equation (4.21), there are only two terms which contain $q$. Write $q=q_{0}+h_{\mathrm{r} f} q_{1}$, where $0 \leq q_{0}<N$. Since $\omega_{0} T_{0}=2 \pi$, the exponential term only depends on $q_{0}$. Thus, the $q$ summation can be turned into a summation over $q_{0}$ and $q_{1}$, and the $q_{1}$ summation can be performed. Defining the basis functions

$$
\tilde{D}_{r a}^{\gamma \beta}(\Omega)=e^{i \Omega r T_{0} / h_{r \delta}} D_{r a}^{\gamma \beta}(\Omega),
$$

the eigenvalue equation (4.21) becomes

$$
\tilde{D}_{n b}^{\gamma \beta}(\Omega)=-\frac{i r_{0} c^{2}}{\gamma_{0} L^{2}} \sum_{\alpha a k r} N_{r} e^{-2 \pi i k r / h_{r f}} \sum_{q_{0}=0}^{N-1} e^{-2 \pi i q_{0}(n-r) / h_{\mathrm{r}}} \tilde{Z}_{q_{0} k b a n r}^{\gamma \beta \alpha}(\Omega) \tilde{D}_{r a}^{\beta \alpha}\left(\Omega+k \omega_{0}\right),
$$

where

$$
\tilde{Z}_{q_{0} k b a n r}^{\gamma \beta \alpha}(\Omega)=\sum_{q_{1}} Z_{q 0+q_{1} h_{r f} k b a n r}^{\gamma \beta \alpha}(\Omega)
$$

Next, assume that the distribution $\Psi_{r 0}(J)$ is independent of the bunch number $r$. This is a good assumption at low current, where the bunch length is dominated by radiation excitation and not potential-well distortion [30]. This eliminates the last dependence of the $R_{q p r}^{\beta \alpha}\left(\Omega, s, s^{\prime}\right)$ defined in equation (4.11) on $r$, and thus the $B_{q r a}^{\beta \alpha}(\Omega, s)$ and the $C_{q r a}^{\beta \alpha}(\Omega, s)$ of equation (4.12) become independent of $r$. It then follows that $\tilde{Z}_{q k b a n r}^{\gamma \beta \alpha}(\Omega)$ is independent of $n$ and $r$; call this $\hat{Z}_{q k b a}^{\gamma \beta \alpha}(\Omega)$. Define the basis functions

$$
\hat{D}_{q_{0} a}^{\beta \alpha}(\Omega)=\sum_{r} N_{r} e^{2 \pi i g_{0} r / h_{s}} \tilde{D}_{r a}^{\beta \alpha}(\Omega)
$$

then the eigenvalue equation (4.24) becomes

$$
\hat{D}_{p_{0} b}^{\gamma \beta}(\Omega)=-\frac{i r_{0} c^{2}}{\gamma_{0} L^{2}} \sum_{\alpha a k q_{0}}\left(\sum_{n} N_{n} e^{2 \pi i\left(p_{0}-q_{0}\right) n / h_{\mathrm{rt}}}\right) \hat{z}_{q 0 k b a}^{\gamma \beta \alpha}(\Omega) \hat{D}_{q_{0}-k, a}^{\beta \alpha}\left(\Omega+k \omega_{0}\right) .
$$

The most interesting case of this is usually the symmetric bunch case, when $N_{n}$ is independent of $n$. In this case, the sum over $n$ is only nonzero when $p_{0}=q_{0}$.

Both equations (4.24) and (4.27) are potentially useful; the latter if the bunches are nearly symmetric, the former when this is not true.

Note that in all the above and subsequent equations, $h_{\mathrm{rf}}$ may be replaced by $M$, the minimum number of bunch buckets required to have $M$ buckets equally spaced, with none of the buckets outside of those $M$ buckets containing particles. Note that $h_{\mathrm{rf}}$ is therefore an integer multiple of $M$.

\subsection{Linear Lattice without Dispersion}

Begin by assuming a linear lattice without dispersion (except for the contribution of dispersion to the momentum compaction factor described below). Thus, the lattice part of the Hamiltonian will be given by equation (3.54). Appendices A.3 and A.4 describe the transformation of such a Hamiltonian to action-angle variables. The resulting transformations give the functions

$$
\begin{aligned}
& \hat{x}(\theta, J, s)=\sqrt{2 J_{x} \beta_{x}(s)} \cos \left(\theta_{x}+\psi_{x}(s)-\frac{\omega_{x} s}{\beta_{0} c}\right) \\
& \hat{y}(\theta, J, s)=\sqrt{2 J_{y} \beta_{y}(s)} \cos \left(\theta_{y}+\psi_{y}(s)-\frac{\omega_{y} s}{\beta_{0} c}\right) \\
& \hat{\tau}(\theta, J, s)=\sqrt{2 J_{\tau} \beta_{\tau}(s)} \cos \left(\theta_{\tau}+\psi_{\tau}(s)-\frac{\omega_{\tau} s}{\beta_{0} c}\right)
\end{aligned}
$$

The determination of the functions $\beta_{x, y, r}, \psi_{x, y, \tau}$, and the numbers $\omega_{x, y, \tau}$ is discussed in appendices A.3 and A.4. In particular, $\beta_{r}$ is given by equation (A.28), with

$$
\begin{gathered}
k_{p}(s)=-\frac{1}{\beta_{0}^{2} c^{2}}\left(h(s) \eta(s)-\frac{1}{\gamma_{0}^{2}}\right) \\
\hat{K}_{p}=-\frac{\eta_{C} L}{\beta_{0}^{2} c^{2}} \\
k_{q}=-\frac{e \hat{V} \omega_{\mathrm{rf}}}{p_{0}} .
\end{gathered}
$$

$\eta_{C}$ is known as the momentum compaction $[11,68]$.

All potential-well distortion effects will be ignored. Thus, all the approximations of section 4.2 hold.

\subsubsection{Computation of $R, B$, and $C$}

The next step in the analysis of the system for a linear lattice without dispersion is to compute the functions $R, B$, and $C$ as defined in equations (4.11) and (4.12).

From the definition of $R_{q p r}^{\beta \alpha}$ in equation (4.11), the form of the functions $x$ and $y$ given by equations (4.28) and (4.29), and the form for the inverse of $G$ given in section 4.1.2, it follows that $R_{q p}^{\beta \alpha}$ is zero unless $\beta=\alpha$ (notice that the bunch number index has been dropped as per section 4.2). Using the method of section 4.1.2 to invert $G$, the remaining $R_{q p}^{\beta \alpha}$ are

$$
R_{q p}^{00}\left(\Omega, s, s^{\prime}\right)=-\frac{i}{\omega_{\tau}} Q P \csc \pi \frac{\Omega}{\omega_{\tau}} \int_{-\pi}^{\pi} d_{0}^{\prime}(\Delta) \sin \varphi e^{-i \Omega \theta^{\prime} / \omega_{\tau}} d \theta^{\prime}
$$




$$
\begin{gathered}
R_{q p}^{\alpha \alpha}\left(\Omega, s, s^{\prime}\right)=\frac{\sqrt{\beta_{\alpha}(s) \beta_{\alpha}\left(s^{\prime}\right)}}{4 \omega_{\tau}} \int_{-\pi}^{\pi}\{ \\
e^{i\left[\Delta \psi_{\alpha}\left(s^{\prime}\right)-\Delta \psi_{\alpha}(s)\right]_{e}-i\left(\Omega-\omega_{\alpha}\right) \theta^{\prime} / \omega_{\tau}} \csc \pi \frac{\Omega-\omega_{\alpha}}{\omega_{\tau}}\left[\rho(\Delta)-2 i d_{\alpha}^{\prime}(\Delta) Q P \sin \varphi\right] \\
\left.-e^{-i\left[\Delta \psi_{\alpha}\left(s^{\prime}\right)-\Delta \psi_{\alpha}(s)\right]} e^{-i\left(\Omega+\omega_{\alpha}\right) \theta^{\prime} / \omega_{\tau}} \csc \pi \frac{\Omega+\omega_{\alpha}}{\omega_{\tau}}\left[\rho(\Delta)+2 i d_{\alpha}^{\prime}(\Delta) Q P \sin \varphi\right]\right\} d \theta^{\prime}
\end{gathered}
$$

where

$$
\begin{gathered}
\Delta=\Delta(Q, P, \varphi) \\
Q=\sqrt{\beta_{\tau}(s)}\left(q \omega_{0}+\Omega\right) \\
P=\sqrt{\beta_{\tau}\left(s^{\prime}\right)}\left(p \omega_{0}+\Omega\right) \\
\varphi=\Delta \psi_{\tau}\left(s^{\prime}\right)-\Delta \psi_{\tau}(s)+\theta^{\prime} \\
\Delta \psi_{\alpha}(s)=\psi_{\alpha}(s)-\frac{\mu_{\alpha} s}{L} \\
\Delta(Q, P, \varphi)=Q^{2}+P^{2}+2 Q P \cos \varphi \\
\rho(x)=8 \pi^{3} \int \Psi_{0}(J) J_{0}\left(\sqrt{2 J_{\tau} x}\right) d^{3} J \\
d_{0}(x)=8 \pi^{3} \int \frac{\partial \Psi_{0}}{\partial J_{\tau}} J_{0}\left(\sqrt{2 J_{\tau} x}\right) d^{3} J \\
d_{\alpha}(x)=8 \pi^{3} \int J_{\alpha} \frac{\partial \Psi_{0}}{\partial J_{\tau}} J_{0}\left(\sqrt{2 J_{\tau} x}\right) d^{3} J
\end{gathered}
$$

The index $\alpha$ is either $x$ or $y$; throughout the rest of this paper, Greek indices will have either this meaning or will designate all of $0, x$, and $y$. The meaning will be clear from the context.

Note that the $d_{\alpha}$ involve the derivative of the distribution with respect to $J_{\tau}$. If the distribution is only known once the momenta have been integrated out, for instance, the distribution as a function of $J$ can be found as described in appendix $B$.

The $B$ and $C$ defined in equation (4.12) can be computed for the $R$ 's defined in equations (4.34)-(4.35). The procedure is to perform a Taylor expansion in $x$ of the quantities

$$
\vec{f}_{\alpha}(x Q, x P, \varphi)=\frac{f_{\alpha}(x Q, x P, \varphi) f_{\alpha}(0,0, \varphi)}{f_{\alpha}(x Q, 0, \varphi) f_{\alpha}(0, x P, \varphi)}
$$

where

$$
\begin{gathered}
f_{0}(Q, P, \varphi)=d_{0}^{\prime}(\Delta(Q, P, \varphi)) \\
f_{\alpha}(Q, P, \varphi)=\rho(\Delta(Q, P, \varphi))-2 i Q P \sin \varphi d_{\alpha}^{\prime}(\Delta(Q, P, \varphi)) .
\end{gathered}
$$

The Taylor expansion of $\bar{f}_{\alpha}(x Q, x P, \varphi)$ about $x=0$ evaluated at $x=1$ can be written in the form

$$
\sum_{k l n}\left[f_{\lambda k l n} P^{n+2 k} Q^{n+2 l} \cos ^{n} \varphi-i g_{\lambda k l n} P^{n+2 k} Q^{n+2 l} \cos ^{n-1} \varphi \sin \varphi\right]
$$

The $\theta^{\prime}$ integrals in equations (4.34) and (4.35) can now be performed. Since

$$
\begin{aligned}
\int_{-\pi}^{\pi} e^{-i \lambda \theta} \cos ^{m}(\theta+\phi) d \theta & =\sin \pi \lambda \frac{(-1)^{m}}{2^{m-1}} \sum_{k=0}^{m}\left(\begin{array}{c}
m \\
k
\end{array}\right) \frac{e^{-i(m-2 k) \phi}}{\lambda+m-2 k} \\
\int_{-\pi}^{\pi} e^{-i \lambda \theta} \cos ^{m-1}(\theta+\phi) \sin (\theta+\phi) d \theta & =\frac{i}{m} \sin \pi \lambda \frac{(-1)^{m}}{2^{m-1}} \sum_{k=0}^{m}\left(\begin{array}{c}
m \\
k
\end{array}\right)(m-2 k) \frac{e^{-i(m-2 k) \phi}}{\lambda+m-2 k},
\end{aligned}
$$

the resulting expressions for the $R$ 's are

$$
\begin{aligned}
& R_{q p}^{00}\left(\Omega, s, s^{\prime}\right)=\frac{d_{0}^{\prime}\left(\left(p \omega_{0}+\Omega\right)^{2} \beta_{\tau}\left(s^{\prime}\right)\right) d_{0}^{\prime}\left(\left(q \omega_{0}+\Omega\right)^{2} \beta_{\tau}(s)\right)}{d_{0}^{\prime}(0)} \\
& \sum_{k l m n}(-1)^{n} \frac{n+1-2 m}{(n+1) 2^{n}}\left(\begin{array}{c}
n+1 \\
m
\end{array}\right) f_{0 k l n} \\
& {\left[\sqrt{\beta_{\tau}\left(s^{\prime}\right)}\left(p \omega_{0}+\Omega\right)\right]^{n+2 k+1}\left[\sqrt{\beta_{\tau}(s)}\left(q \omega_{0}+\Omega\right)\right]^{n+2 l+1} \frac{e^{i(n+1-2 m)\left[\Delta \psi_{\tau}\left(s^{\prime}\right)-\Delta \psi_{\tau}(s)\right]}}{\Omega-(n+1-2 m) \omega_{\tau}}} \\
& R_{q p}^{\alpha \alpha}\left(\Omega, s, s^{\prime}\right)=\frac{\sqrt{\beta_{\alpha}(s) \beta_{\alpha}\left(s^{\prime}\right)} \frac{\rho\left(\beta_{\tau}(s)\left(q \omega_{0}+\Omega\right)^{2}\right) \rho\left(\beta_{\tau}\left(s^{\prime}\right)\left(p \omega_{0}+\Omega\right)^{2}\right)}{4}}{\rho(0)} \\
& \sum_{k l m n} \frac{(-1)^{n}}{2^{n-1}}\left(\begin{array}{c}
n \\
m
\end{array}\right)\left(f_{\alpha k l n}+\frac{n-2 m}{n} g_{\alpha k l n}\right)\left[\sqrt{\left.\beta_{\tau}\left(s^{\prime}\right)\left(p \omega_{0}+\Omega\right)\right]^{n+2 k}}\left[\sqrt{\beta_{\tau}(s)}\left(q \omega_{0}+\Omega\right)\right]^{n+2 l}\right. \\
& {\left[\frac{e^{i\left[\Delta \psi_{\alpha}\left(s^{\prime}\right)-\Delta \psi_{\alpha}(s)-(n-2 m)\left\{\Delta \psi_{\tau}\left(s^{\prime}\right)-\Delta \psi_{r}(s)\right]\right\}}}{\Omega-\left[\omega_{\alpha}-(n-2 m) \omega_{\tau}\right]}-\frac{e^{-i\left\{\Delta \psi_{\alpha}\left(s^{\prime}\right)-\Delta \psi_{\alpha}(s)-(n-2 m)\left[\Delta \psi_{r}\left(s^{\prime}\right)-\Delta \psi_{\tau}(s)\right)\right\}}}{\Omega+\left[\omega_{\alpha}-(n-2 m) \omega_{r}\right]}\right] .}
\end{aligned}
$$

The $m$ sum in (4.51) extends from 0 to $n+1$, while it extends from 0 to $n$ in (4.52).

To separate the $R$ 's into $B$ 's and $C$ 's as described in equation (4.12), the following factorizations are necessary:

$$
\begin{gathered}
f_{0 k l n}=\sum_{d} c_{0 k n d} b_{0 l n d} \\
f_{\alpha k l n}+\frac{n-2 m}{n} g_{a k l n}=\sum_{d} c_{\alpha k n m d} b_{\alpha i n m d} .
\end{gathered}
$$


This factorization is possible in general. One way to do it is to factorize the matrix with indices $k$ and $l$ using an LU decomposition [29].

Thus, the $B$ 's and $C$ 's that result from equations (4.51) and (4.52) are

$$
\begin{aligned}
& B_{q[m n d]}^{00}(\Omega, s)= {\left[\sqrt{\beta_{\tau}(s)}\left(q \omega_{0}+\Omega\right)\right]^{n+1} b_{0 n d}\left(\beta_{\tau}(s)\left(q \omega_{0}+\Omega\right)^{2}\right) } \\
& e^{-i(n+1-2 m) \Delta \psi_{\tau}(s)} d_{0}^{\prime}\left(\beta_{\tau}(s)\left(q \omega_{0}+\Omega\right)^{2}\right) \\
& C_{q[m n d]}^{00}(\Omega, s)=(-1)^{n} \frac{n+1-2 m}{(n+1) 2^{n}}\left(\begin{array}{c}
n+1 \\
m
\end{array}\right)\left[\sqrt{\beta_{\tau}(s)}\left(q \omega_{0}+\Omega\right)\right]^{n+1} c_{0 n d}\left(\beta_{\tau}(s)\left(q \omega_{0}+\Omega\right)^{2}\right) \\
& \cdots \\
&-e^{i(n+1-2 m) \Delta \psi_{r}(s) \frac{d_{0}^{\prime}\left(\beta_{\tau}(s)\left(q \omega_{0}+\Omega\right)^{2}\right)}{d_{0}^{\prime}(0)}} \frac{1}{\Omega-(n+1-2 m) \omega_{\tau}}
\end{aligned}
$$

$B_{q[k n m d \pm]}^{\alpha \alpha}=\sqrt{\beta_{\alpha}(s)} \rho\left(\beta_{r}(s)\left(q \omega_{0}+\Omega\right)^{2}\right) b_{\alpha n m d}\left(\beta_{r}(s)\left(q \omega_{0}+\Omega\right)^{2}\right)$

$$
\left(\sqrt{\beta_{\tau}(s)}\left(q \omega_{0}+\Omega\right)\right)^{n} e^{\mp i\left[\Delta \psi_{\alpha}(s)-(n-2 m) \Delta_{\tau}(s)\right]},
$$

$C_{q[k n m d \pm]}^{\alpha \alpha}= \pm \frac{(-1)^{n}}{2^{n+1}}\left(\begin{array}{c}n \\ m\end{array}\right) \frac{1}{\rho(0)} \sqrt{\beta_{\alpha}(s) \rho} \rho\left(\beta_{\tau}(s)\left(q \omega_{0}+\Omega\right)^{2}\right) c_{\alpha n m d}\left(\beta_{\tau}(s)\left(q \omega_{0}+\Omega\right)^{2}\right)$

$$
\left(\sqrt{\beta_{\tau}(s)}\left(q \omega_{0}+\Omega\right)\right)^{n} \frac{e^{ \pm i\left[\Delta \psi_{\alpha}(s)-(n-2 m) \Delta \psi_{\tau}(s)\right]}}{\Omega \mp\left[\omega_{\alpha}-(n-2 m) \omega_{\tau}\right]}
$$

where

$$
\begin{aligned}
b_{0 n d}(Q) & =\sum_{k} b_{0 k n d} Q^{k} \\
c_{0 n d}(Q) & =\sum_{k} c_{0 k n d} Q^{k} \\
b_{\alpha n m d}(Q) & =\sum_{k} b_{\alpha k n m d} Q^{k} \\
c_{\alpha n m d}(Q) & =\sum_{k} c_{\alpha k n m d} Q^{k}
\end{aligned}
$$

These results can now be used to compute the "effective impedance" $\hat{Z}_{q k b a}^{\alpha \alpha \alpha}(\Omega)$ described in section 4.2. The results are (dropping indices where appropriate)

$$
\begin{gathered}
Z_{q k[m n d]\left[m^{\prime} n^{\prime} d^{\prime}\right]}^{0}(\Omega)=(-1)^{n} \frac{n+1-2 m}{(n+1) 2^{n}}\left(\begin{array}{c}
n+1 \\
m
\end{array}\right) \frac{1}{\Omega-(n+1-2 m) \omega_{\tau}} \\
\int_{0}^{L} d s e^{-2 \pi i k s / L} \frac{\beta_{0} c Z_{\|}\left(q \omega_{0}+\Omega, s\right)}{q \omega_{0}+\Omega}\left[\sqrt{\beta_{\tau}(s)}\left(q \omega_{0}+\Omega\right)\right]^{n+n^{\prime}+2} e^{i \Delta \psi_{\tau}(s)\left[n-2 m-n^{\prime}+2 m^{\prime}\right]} \\
c_{0 n d}\left(\beta_{\tau}(s)\left(q \omega_{0}+\Omega\right)^{2}\right) b_{0 n^{\prime} d^{\prime}}\left(\beta_{\tau}(s)\left(q \omega_{0}+\Omega\right)^{2}\right) \frac{\left[d_{0}^{\prime}\left(\beta_{\tau}(s)\left(q \omega_{0}+\Omega\right)^{2}\right)\right]^{2}}{d_{0}^{\prime}(0)} \\
Z_{q k[m n d \pm]\left[m^{\prime} n^{\prime} d^{\prime} \pm\right]}^{\alpha}(\Omega)= \pm \frac{(-1)^{n}}{2^{n+1}}\left(\begin{array}{c}
n \\
m
\end{array}\right) \frac{1}{\Omega \mp\left[\omega_{\alpha}-(n-2 m) \omega_{\tau}\right]} \\
\int_{0}^{L} d s e^{-2 \pi i k s / L} \beta_{\alpha}(s) Z_{\perp}\left(q \omega_{0}+\Omega, s\right)\left[\sqrt{\beta_{\tau}(s)}\left(q \omega_{0}+\Omega\right)\right]^{n+n^{\prime}} e^{\mp i \Delta \psi_{\tau}(s)\left(n-2 m-n^{\prime}+2 m^{\prime}\right)} \\
c_{\alpha n m d}\left(\beta_{\tau}(s)\left(q \omega_{0}+\Omega\right)^{2}\right) b_{\alpha n^{\prime} m^{\prime} d^{\prime}}\left(\beta_{\tau}(s)\left(q \omega_{0}+\Omega\right)^{2}\right) \frac{\rho^{2}\left(\beta_{\tau}(s)\left(q \omega_{0}+\Omega\right)^{2}\right)}{\rho(0)} \\
Z_{q k[m n d \pm]\left[m^{\prime} n^{\prime} d^{\prime} \mp\right]}^{\alpha}(\Omega)= \pm \frac{(-1)^{n}}{2^{n+1}}\left(\begin{array}{c}
n \\
m
\end{array}\right) \frac{1}{\Omega \mp\left[\omega_{\alpha}-(n-2 m) \omega_{\tau}\right]} \int_{0}^{L} d s e^{-2 \pi i k s / L} \\
\beta_{\alpha}(s) Z_{\perp}\left(q \omega_{0}+\Omega, s\right)\left[\sqrt{\beta_{\tau}(s)}\left(q \omega_{0}+\Omega\right)\right]^{n+n^{\prime}} e^{ \pm i\left[2 \Delta \psi_{\alpha}(s)-\Delta \psi_{\tau}(s)\left(n-2 m+n^{\prime}-2 m^{\prime}\right)\right]} \\
c_{\alpha n m d}\left(\beta_{\tau}(s)\left(q \omega_{0}+\Omega\right)^{2}\right) b_{\alpha n^{\prime} m^{\prime} d^{\prime}}\left(\beta_{\tau}(s)\left(q \omega_{0}+\Omega\right)^{2}\right) \frac{\rho^{2}\left(\beta_{\tau}(s)\left(q \omega_{0}+\Omega\right)^{2}\right)}{\rho(0)}
\end{gathered}
$$

Equations (4.63)-(4.65) demonstrate the assertion made earlier that the single-bunch growth rates and frequency shifts are the average of the multibunch growth rates and frequency shifts. The assertion only holds true in the case of symmetric bunches, if coupling is ignored. The single bunch shift is proportional to the result of summing the $Z_{q}^{\alpha}$... over all integers $q$. The multibunch shift for the mode $q_{0}$ is proportional to the result of summing $Z_{q}^{\alpha}$.. over all integers $q=q_{0}+q_{1} M$, where $q_{1}$ is an integer and $M$ is the number of bunches. The proportionality constant for the multibunch case is just the proportionality constant for the single bunch case times $M$ (see the discussion of symmetric bunches at the end of section 4.2). Thus, the sum of the multibunch shifts should be $M$ times the single bunch shift, assuming that a "bunch" in either case contains the same number of particles. Thus, under these conditions, the single-bunch frequency shift and growth rate gives the average of the multibunch frequency shifts and growth rates for a given type of mode. 


\subsubsection{Gaussian Bunches}

Consider the case where the unperturbed distribution is Gaussian in the coordinates. This is the expected situation for electron machines and some proton machines which have significant radiation damping [30]. Then $\Psi_{0}(J)$ takes the form

$$
\Psi_{0}(J)=\frac{1}{8 \pi^{3}} \frac{1}{\Sigma_{x} \Sigma_{y} \Sigma_{\tau}} e^{-J_{x} / \Sigma_{x}} e^{-J_{y} / \Sigma_{y}} e^{-J_{\tau} / \Sigma_{\tau}}
$$

Then $\rho(x), d_{0}(x)$, and $d_{\alpha}(x)$ (as defined in equations (4.42), (4.43), and (4.44) respectively) are

$$
\begin{gathered}
\rho(x)=e^{-x \Sigma_{\tau} / 2} \\
d_{0}(x)=-\frac{1}{\Sigma_{\tau}} e^{-x \Sigma_{\tau} / 2} \\
d_{\alpha}(x)=-\frac{\Sigma_{\alpha}}{\Sigma_{\tau}} e^{-x \Sigma_{\tau} / 2}
\end{gathered}
$$

and thus the $\bar{f}_{\alpha}(Q, P, \varphi)$ are

$$
\vec{f}_{0}(Q, P, \varphi)=e^{-\Sigma_{\tau} Q P \cos \varphi}
$$

$$
\vec{f}_{\alpha}(Q, P, \varphi)=e^{-\Sigma_{r} Q P \cos \varphi}\left(1-i \Sigma_{\alpha} Q P \sin \varphi\right) .
$$

Therefore $f_{\alpha k l n}$ and $g_{\alpha k l n}$ are given by

$$
\begin{gathered}
f_{\alpha k l n}=\delta_{k 0} \delta_{l 0}(-1)^{n} \frac{1}{n !} \Sigma_{\tau}^{n} \\
g_{\alpha k l n}=\Sigma_{\alpha} \Sigma_{\tau}^{n-1} \delta_{k 0} \delta_{l 0}(-1)^{n-1} \frac{1}{(n-1) !}
\end{gathered}
$$

and thus

$$
\begin{gathered}
c_{0 n d}(Q)=\delta_{d 0}(-1)^{n} \frac{1}{n !} \Sigma_{\tau}^{n / 2} \\
b_{0 n d}(Q)=\delta_{d 0} \Sigma_{\tau}^{n / 2} \\
c_{\alpha n m d}(Q)=\delta_{d 0}(-1)^{n} \frac{1}{n !} \Sigma_{\tau}^{n / 2}\left[1-\frac{\Sigma_{\alpha}}{\Sigma_{\tau}}(n-2 m)\right] \\
b_{\alpha n m d}(Q)=\delta_{d 0} \Sigma_{\tau}^{n / 2} .
\end{gathered}
$$

Therefore, the effective impedances are given by

$$
\begin{aligned}
& Z_{q k[m n]\left[m^{\prime} n^{\prime}\right]}^{0}(\Omega)=\frac{n+1-2 m}{(n+1) ! 2^{n+1} \Sigma_{\tau}}\left(\begin{array}{c}
n+1 \\
m
\end{array}\right) \frac{1}{\Omega-(n+1-2 m) \omega_{\tau}} \\
& \int_{0}^{L} d s e^{-2 \pi i k s / L} \frac{\beta_{0} c Z_{\|}\left(q \omega_{0}+\Omega, s\right)}{q \omega_{0}+\Omega} e^{i \Delta \psi_{\tau}(s)\left(n-2 m-n^{\prime}+2 m^{\prime}\right)} \\
& {\left[\sigma_{\tau}(s)\left(q \omega_{0}+\Omega\right)\right]^{n+n^{\prime}+2} e^{-\sigma_{\tau}^{2}(s)\left(q \omega_{0}+\Omega\right)^{2}} }
\end{aligned}
$$

$$
\begin{array}{r}
Z_{q k[m n \pm]\left[m^{\prime} n^{\prime} \pm\right]}^{\alpha}= \pm \frac{1}{2^{n+1} n !}\left(\begin{array}{c}
n \\
m
\end{array}\right)\left[1-\frac{\Sigma_{\alpha}}{\Sigma_{\tau}}(n-2 m)\right] \frac{1}{\Omega \mp\left[\omega_{\alpha}-(n-2 m) \omega_{\tau}\right]} \\
\int_{0}^{L} d s e^{-2 \pi i k s / L} \beta_{\alpha}(s) Z_{\perp}\left(q \omega_{0}+\Omega, s\right) e^{\mp i \Delta \psi_{\tau}(s)\left(n-2 m-n^{\prime}+2 m^{\prime}\right)} \\
{\left[\sigma_{\tau}(s)\left(q \omega_{0}+\Omega\right)\right]^{n+n^{\prime}} e^{-\sigma_{\tau}^{2}(s)\left(q \omega_{0}+\Omega\right)^{2}}}
\end{array}
$$

$$
\begin{array}{r}
Z_{q k[m n \pm]\left[m^{\prime} n^{\prime} \mp\right]}^{\alpha}= \pm \frac{1}{2^{n+1} n !}\left(\begin{array}{l}
n \\
m
\end{array}\right)\left[1-\frac{\Sigma_{\alpha}}{\Sigma_{\tau}}(n-2 m)\right] \frac{1}{\Omega \mp\left[\omega_{\alpha}-(n-2 m) \omega_{r}\right]} \\
\int_{0}^{L} d s e^{-2 \pi i k s / L} \beta_{\alpha}(s) Z_{\perp}\left(q \omega_{0}+\Omega, s\right) e^{ \pm i\left[2 \Delta \psi_{\alpha}(s)-\Delta \psi_{r}(s)\left(n-2 m+n^{\prime}-2 m^{\prime}\right)\right]} \\
{\left[\sigma_{\tau}(s)\left(q \omega_{0}+\Omega\right)\right]^{n+n^{\prime}} e^{-\sigma_{\tau}^{2}(s)\left(q \omega_{0}+\Omega\right)^{2}},}
\end{array}
$$

where $\sigma_{\tau}(s)=\sqrt{\Sigma_{\tau} \beta_{\tau}(s)}$ is the r.m.s. bunch half-length as a function of $s$. Note that the $d$ indices have been suppressed since they don't enter. These results are consistent with the formulas given in [70], if the approximations therein are used.

It is useful to put equations (4.27) and (4.78)-(4.80) in terms of quantities more commonly used in accelerator physics. It is also useful to regroup terms in this equations. The results are

$$
\begin{gathered}
{\left[\frac{\Omega}{\omega_{0}}+(n+1-2 m) \nu_{s}\right] \hat{D}_{p_{0}[m n]}^{0}=-i \frac{\eta_{C}}{4 \pi^{2} \beta_{0}^{2}} \frac{e}{E_{0}} \frac{L^{2}}{\sigma_{\tau}^{2}\left(s_{\mathrm{rf}}\right) \beta_{0}^{2} c^{2}} \csc 2 \pi \nu_{s}\left(\begin{array}{c}
n+1 \\
m
\end{array}\right)} \\
\frac{n+1-2 m}{(n+1) ! 2^{n+1}} \cdot \sum_{m^{\prime} n^{\prime} k q_{0}}\left(\sum_{r} I_{r} e^{2 \pi i\left(p_{0}-q_{0}\right) r / h_{r f}}\right) Z_{k[n m]\left[n^{\prime} m^{\prime}\right], \mathrm{eff}}^{\|}\left(q_{0} \omega_{0}+\Omega\right) \hat{D}_{q_{0}-k,\left[m^{\prime} n^{\prime}\right]}^{0}\left(\Omega+k \omega_{0}\right)
\end{gathered}
$$$$
Z_{k[n m]\left[n^{\prime} m^{\prime}\right], \mathrm{eff}}^{\|}(\omega)=\sum_{q_{1}} \int_{0}^{L} d s e^{-2 \pi i k s / L} \frac{Z_{\|\|}\left(h_{\mathrm{rf}} q_{1} \omega_{0}+\omega, s\right)}{h_{\mathrm{rf}} q_{1}+\omega / \omega_{0}} e^{i \Delta \psi_{r}(s)\left(n-2 m-n^{\prime}+2 m^{\prime}\right)}
$$

$$
\left[\sigma_{r}(s)\left(h_{\mathrm{rf}} q_{1} \omega_{0}+\omega\right)\right]^{n+n^{\prime}+2} e^{-\sigma_{\tau}^{2}(s)\left(h_{\mathrm{r}} q_{1} \omega_{0}+\omega\right)^{2}}
$$

$$
\begin{gathered}
\left\{\frac{\Omega}{\omega_{0}} \mp\left[\nu_{\alpha}+(n-2 m) \nu_{s}\right]\right\} \hat{D}_{p_{0}[m n \pm]}^{\alpha}=\mp i \frac{1}{2 \pi \beta_{0}^{2}} \frac{e}{E_{0}}\left(\begin{array}{c}
n \\
m
\end{array}\right) \frac{1}{2^{n+1} n !} \\
{\left[1-\frac{\epsilon_{\alpha} \eta_{C} L}{\sigma_{\tau}^{2}\left(s_{\mathrm{rf}}\right) \beta_{0}^{2} c^{2}}(n-2 m) \csc 2 \pi \nu_{s}\right] \sum_{m^{\prime} n^{\prime} k q_{0}}\left(\sum_{\tau} I_{r} e^{2 \pi i\left(p_{0}-q_{0}\right) r / h_{\mathrm{sf}}}\right)} \\
{\left[Z_{k[m n \pm]\left[m^{\prime} n^{\prime} \pm\right], \mathrm{eff}}^{\alpha}\left(q_{0} \omega_{0}+\Omega\right) D_{q_{0}-k,\left[m^{\prime} n^{\prime} \pm\right]}^{\alpha}\left(\Omega+k \omega_{0}\right)\right.} \\
\left.+Z_{k[m n \pm]\left[m^{\prime} n^{\prime} \mp\right], \mathrm{eff}}^{\alpha}\left(q_{0} \omega_{0}+\Omega\right) D_{q_{0}-k\left[m^{\prime} n^{\prime} \mp\right]}^{\alpha}\left(\Omega+k \omega_{0}\right)\right]
\end{gathered}
$$




$$
\begin{aligned}
& Z_{k[n m \pm]\left[n^{\prime} m^{\prime} \pm\right], \text { eff }}^{\alpha}(\omega)=\sum_{q_{1}} \int_{0}^{L} d s e^{-2 \pi i k s / L} \beta_{\alpha}(s) Z_{\perp}\left(h_{\mathrm{rf}} q_{1} \omega_{0}+\omega, s\right) \\
& e^{\mp i \Delta \psi_{r}(s)\left(n-2 m-n^{\prime}+2 m^{\prime}\right)}\left[\sigma_{\tau}(s)\left(h_{\mathrm{rf}} q_{1} \omega_{0}+\omega\right)\right]^{n+n^{\prime}} e^{-\sigma_{\tau}^{2}(s)\left(h_{\mathrm{r}} q_{1} \omega_{0}+\omega\right)^{2}} \\
& Z_{k\left[n m \pm \|\left(n^{\prime} m^{\prime} \mp\right], \mathrm{eff}\right.}^{\alpha}(\omega)=\sum_{q_{1}} \int_{0}^{L} d s e^{-2 \pi i k s / L} \beta_{\alpha}(s) Z_{\perp}\left(h_{\mathrm{rf}} q_{1} \omega_{0}+\omega, s\right) \\
& e^{ \pm i\left[2 \Delta \psi_{\alpha}(s)-\Delta \psi_{r}(s)\left(n-2 m+n^{\prime}-2 m^{\prime}\right)\right]}\left[\sigma_{\tau}(s)\left(h_{\mathrm{r} f} q_{1} \omega_{0}+\omega\right)\right]^{n+n^{\prime}} e^{-\sigma_{\tau}^{2}(s)\left(h_{\mathrm{r}} q_{1} \omega_{0}+\omega\right)^{2}} .
\end{aligned}
$$

Here $\nu_{\alpha}$ is the betatron tune in the appropriate plane, and $\nu_{s}$ is the synchrotron tune. Note that above transition [68], $\omega_{\tau}$ is negative; $\nu_{s}$ has been chosen to be positive above transition. Also, $\epsilon_{\alpha}$ is the transverse emittance [68], and $I_{r}$ is the current in bunch $r$. Note also that the bunch length at the cavity $\sigma_{\tau}\left(s_{\mathrm{rf}}\right)$ is the longest bunch length at any point in the ring.

Computations can generally be performed assuming that $\sigma_{\tau}$ is constant and that $\Delta \psi_{\tau}$ is zero, since the synchrotron tune is generally very small (see discussion in appendix A.4).

Also, since the interesting effects from this method are seen when the mode shifts are comparable with the synchrotron tune, it follows that ignoring the cross terms from equation (4.85) results in relative errors of order $\nu_{s} / \nu_{\alpha}$, which is a small quantity for most accelerators. This can be seen by considering perturbation theory [61]. Assume that the problem without cross terms has been solved. Then the matrix to find the eigenvalues of takes the form

$$
\left[\begin{array}{cc}
D_{11} & U_{11}^{-1} A_{12} U_{22} \\
U_{22}^{-1} A_{21} U_{11} & D_{22}
\end{array}\right]
$$

where the $D$ 's are diagonal, and $U_{i i}$ diagonalized $A_{i i}$ in the original matrix. The lowest order corrections to the eigenvalues in the $D_{i i}$ are then

$$
\frac{\Omega}{\omega_{0}}-D_{11, k k}=\sum_{l \neq k} \frac{\left(U_{22}^{-1} A_{21} U_{11}\right)_{l k}\left(U_{11}^{-1} A_{12} U_{22}\right)_{k l}}{D_{11, k k}-D_{22, l l}}
$$

Since the $A_{i j}$ are of order $\nu_{s}$, and to lowest order $\left|D_{11, k k}-D_{22, l l}\right|$ is $2 \nu_{\alpha}$, this gives corrections of order $\nu_{s}^{2} / \nu_{\alpha}$. Since the uncorrected shifts are also comparable to $\nu_{s}$, the relative correction is of order $\nu_{s} / \nu_{\alpha}$. Throughout this argument, the variation of (4.84) and (4.85) with $\Omega$ has been ignored; this is an excellent approximation as long as there are no resonances whose width is comparable to or less than $\nu_{s}$ (which is usually the case in the transverse plane).

\subsection{Feedback}

Now, add feedback to this formalism. While all the equations written down will contain only one feedback system, an arbitrary number of feedback systems may be added, and these results will carry through in the obvious way. Beginning with the extra terms in the Hamiltonian (3.64), the Hamiltonian in terms of the impedance (4.2) gets the additional terms

$$
\begin{array}{r}
-\frac{i r_{0}}{2 \pi \beta_{0}^{2} \gamma_{0}} \sum_{n p} N_{n} e^{-2 \pi i p \Delta s / L} \int\left(\beta_{0} c \frac{Z_{\|}^{\mathrm{FB}}\left(p \omega_{0}+\Omega^{\prime}, s\right)}{p \omega_{0}+\Omega^{\prime}}\right. \\
+Z_{\perp}^{\mathrm{FB}}\left(p \omega_{0}+\Omega^{\prime}, s\right)\left[x_{r}(\theta, J, s)+x_{r 0}(s)\right]\left[x_{n}\left(\theta^{\prime}, J^{\prime}, s-\Delta s\right)+x_{n 0}(s-\Delta s)\right] \\
\left.+Z_{\perp}^{\mathrm{FB}}\left(p \omega_{0}+\Omega^{\prime}, s\right)\left[y_{r}(\theta, J, s)+y_{\tau 0}(s)\right]\left[y_{n}\left(\theta^{\prime}, J^{\prime}, s-\Delta s\right)+y_{n 0}(s-\Delta s)\right]\right) \\
e^{-i\left(p \omega_{0}+\Omega^{\prime}\right) \tau_{r}(\theta, J, s)} e^{i\left(p \omega_{0}+\Omega^{\prime}\right) \tau_{n}\left(\theta^{\prime}, J^{\prime}, s-\Delta s\right)} e^{-i\left(p \omega_{0}+\Omega^{\prime}\right) \tau_{r 0}(s)} e^{i\left(p \omega_{0}+\Omega^{\prime}\right) \tau_{n 0}(s-\Delta s)} \\
e^{-i \Omega^{\prime} s / \beta_{0} c} \Psi_{n}\left(\theta^{\prime}, J^{\prime}, \Omega^{\prime}\right) d^{3} \theta^{\prime} d^{3} J^{\prime} d \Omega^{\prime} .
\end{array}
$$

Writing this piece in terms of the $D_{n p}^{\alpha}(\Omega, s)$ (equation (4.6)), the terms

$$
\begin{aligned}
& -\frac{i r_{0}}{2 \pi \beta_{0}^{2} \gamma_{0}} \sum_{\alpha n p} N_{n} e^{-2 \pi i p \Delta s / L} \int z_{\alpha}^{\mathrm{FB}}\left(p \omega_{0}+\Omega^{\prime}, s\right) \\
& \quad\left[f_{r}^{\alpha}(\theta, J, s)+f_{r 0}^{\alpha}(s)\right]\left[D_{n p}^{\alpha}\left(\Omega^{\prime}, s-\Delta s\right)+f_{n 0}^{\alpha}(s) D_{n p}^{0}\left(\Omega^{\prime}, s-\Delta s\right)\right] \\
& \quad e^{-i\left(p \omega_{0}+\Omega^{\prime}\right) \tau_{r}(\theta, J, s)} e^{-i\left(p \omega_{0}+\Omega^{\prime}\right) \tau_{r 0}(s)} e^{i\left(p \omega_{0}+\Omega^{\prime}\right) \tau_{n 0}(s-\Delta s)} e^{-i \Omega^{\prime} s / \beta_{0} c} d \Omega^{\prime}
\end{aligned}
$$

are added to the Hamiltonian of equation (4.7). The $Z_{\alpha}^{\mathrm{FB}}$ are defined to be

$$
Z_{\alpha}^{\mathrm{FB}}(\omega, s)= \begin{cases}\beta_{0} c \frac{Z_{\|}^{\mathrm{FB}}(\omega, s)}{\omega} & \alpha=0 \\ Z_{x}^{\mathrm{FB}}(\omega, s) & \alpha=x \\ Z_{y}^{\mathrm{FB}}(\omega, s) & \alpha=y\end{cases}
$$

Putting the resulting Hamiltonian into the Vlasov equation and writing a self-consistent equation for the $D_{r q}^{\beta}$ gives equation (4.10) with the additional terms

$$
\begin{gathered}
-\frac{i r_{0} c^{2}}{\gamma_{0} L^{2}} \sum_{\alpha k n p} N_{n} e^{-2 \pi i(p-k) \Delta s / L} \int_{0}^{L} d s^{\prime} e^{-2 \pi i k s^{2} / L} e^{-i\left(p \omega_{0}+\Omega\right)\left[\tau_{r}\left(s^{\prime}\right)-\tau_{n 0}\left(s^{\prime}-\Delta s\right)\right]} \\
Z_{\alpha}^{\mathrm{FB}}\left(p \omega_{0}+\Omega, s^{\prime}\right)\left[R_{q p r}^{\beta \alpha}\left(\Omega, s, s^{\prime}\right)+f_{r 0}^{\alpha}\left(s^{\prime}\right) R_{q p r}^{\beta 0}\left(\Omega, s, s^{\prime}\right)\right] \\
{\left[D_{n, p-k}^{\alpha}\left(\Omega+k \omega_{0}, s^{\prime}-\Delta s\right)+f_{n 0}^{\alpha}\left(s^{\prime}-\Delta s\right) D_{n, p-k}^{0}\left(\Omega+k \omega_{0}, s^{\prime}-\Delta s\right)\right]}
\end{gathered}
$$




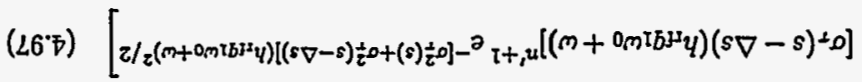

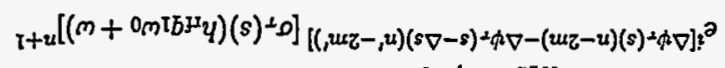

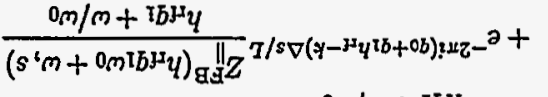

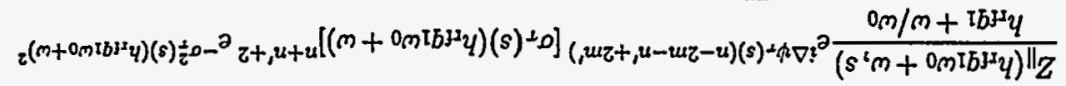

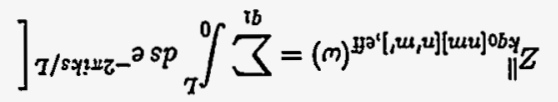

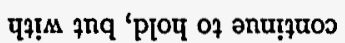

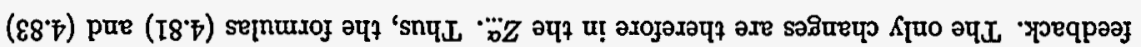

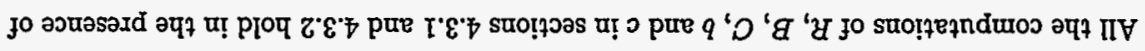

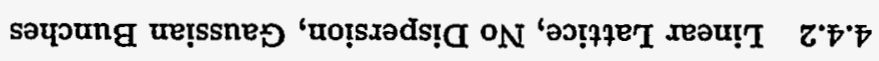

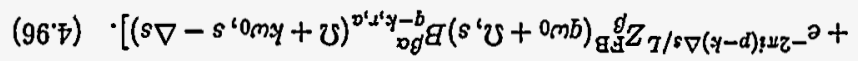

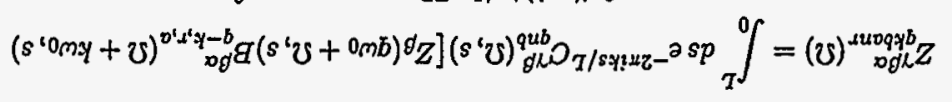

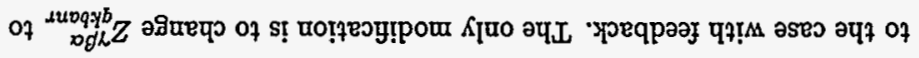

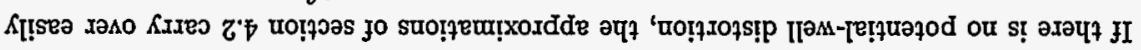

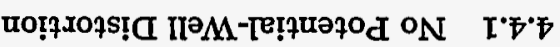

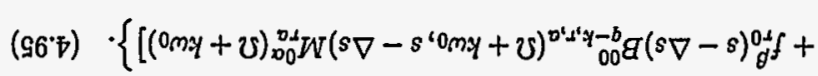

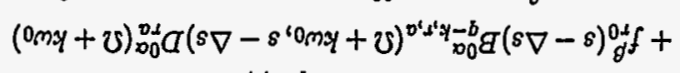

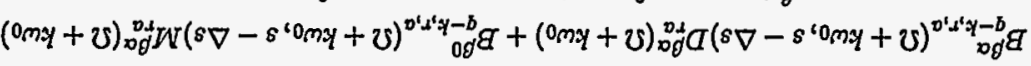

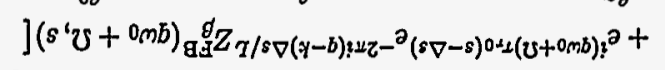

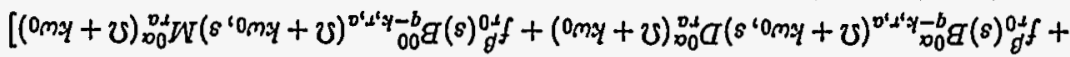

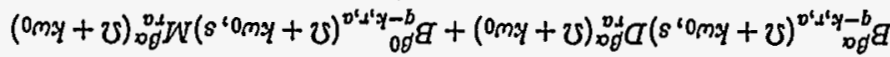

$$
]\left(s^{\prime} v+0 m b\right) d z(s) 0{ }^{2}+(\mho+0 m b) q^{2}
$$

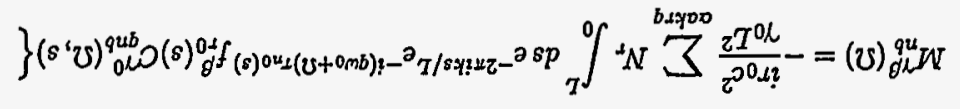

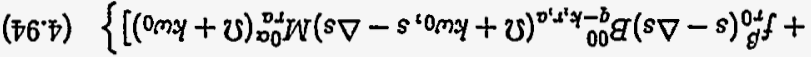

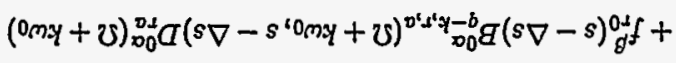

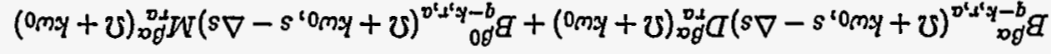

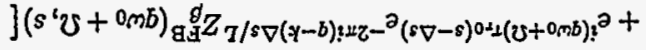

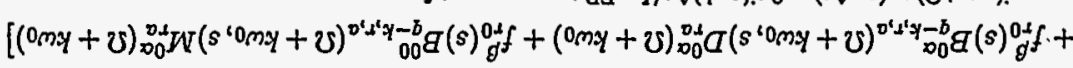

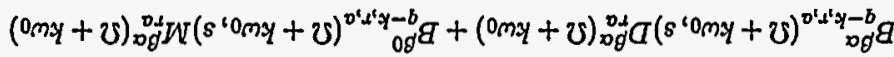
]$\left(s^{\prime} v+0 m b\right) d Z_{(s) 0+\alpha_{1}(v+0 m b) ?^{2}}$

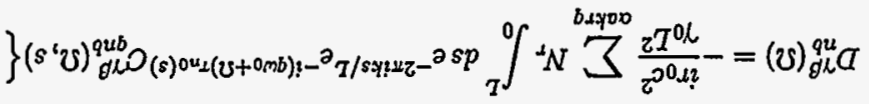

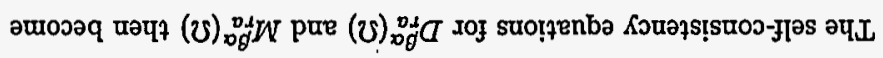

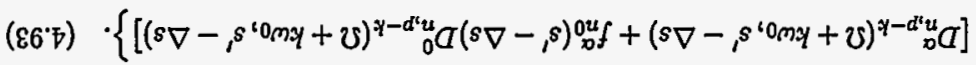

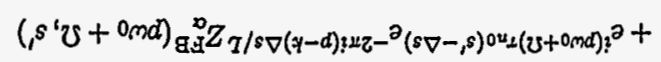

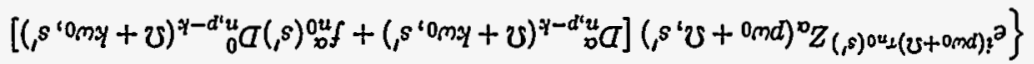

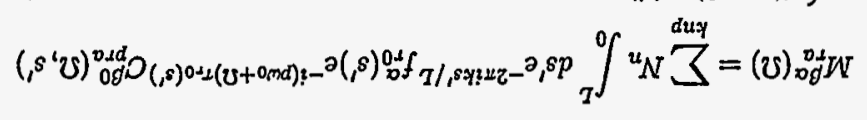

$\left(6^{\prime} \nabla\right)\left\{\left[\left(s \nabla-s^{s} 0 m y+\mho s\right)^{y-d^{d} u} \sigma(s \nabla-s)_{0}^{0 u} f+\left(s \nabla-s^{s} 0 m y+\mho\right)^{y-d^{\prime} u} d\right]\right.$

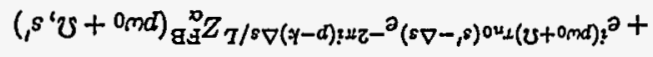

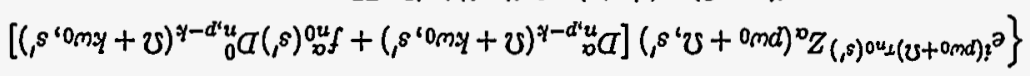

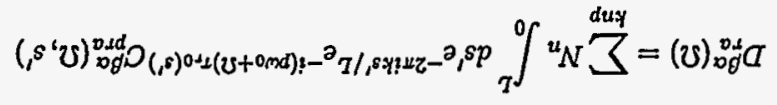

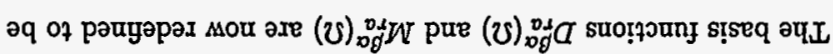

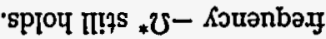

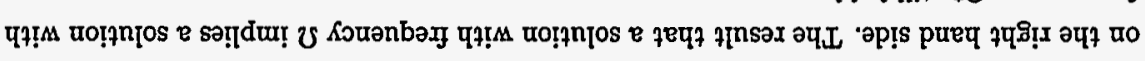




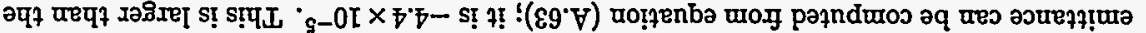

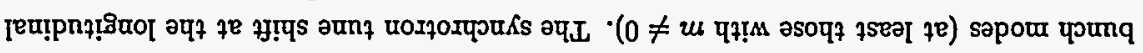

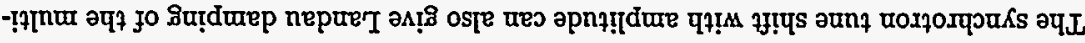

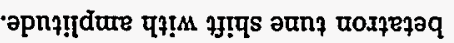

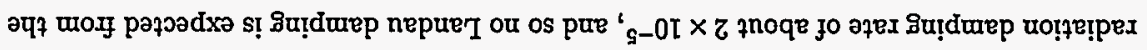

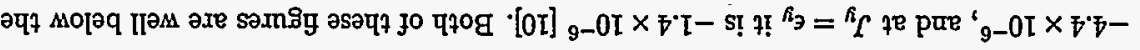

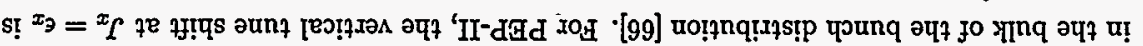

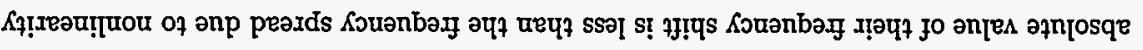

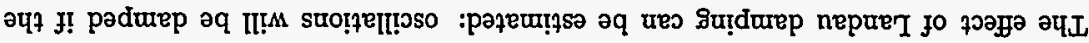

[ [0L' $\left.\angle 9^{\prime} 99^{\prime} 89^{\prime} 69-\angle 9^{\prime} 0 D^{\prime} 8 \varepsilon^{\prime} t \tau\right]$

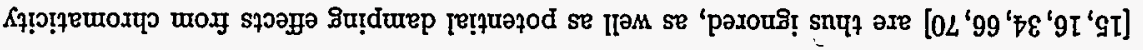

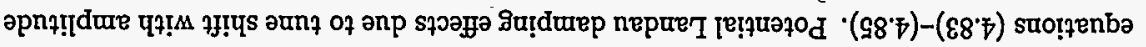

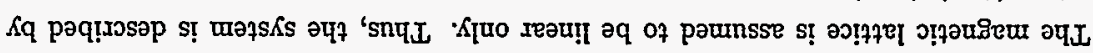

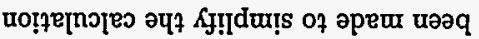

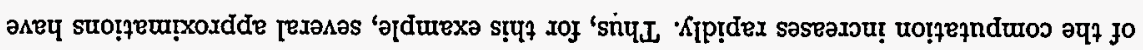

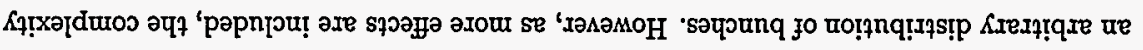

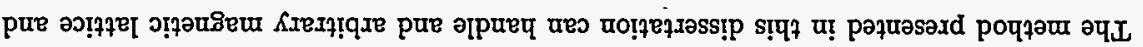

\section{әреIN suo!̣?eu!̣xoIdd}

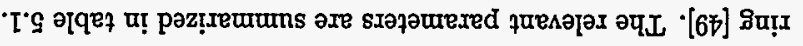

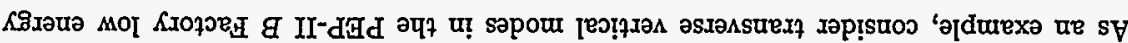

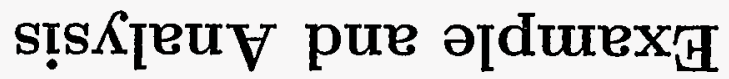

g ләұðечว

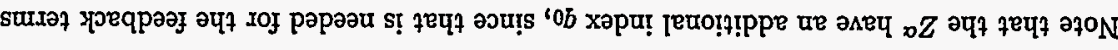

$\left(66^{\circ}\right.$ )

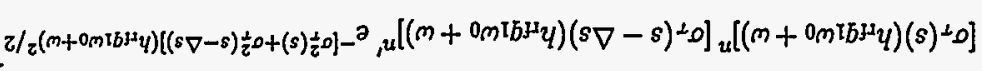

$\left[(, u z-, u)(s \nabla-s)+\phi \nabla-(u z-u)(s)^{\perp} \phi \nabla-(s \nabla-s)^{\circ} \phi \nabla+(s){ }^{\infty} \phi \nabla\right] ! F^{2}$

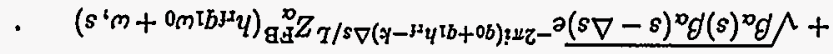

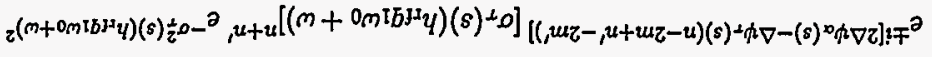

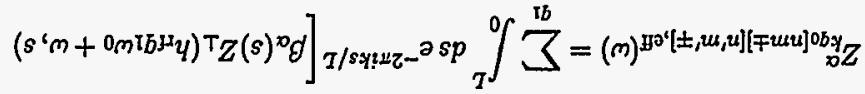

$\left(86^{\circ} 7\right)$

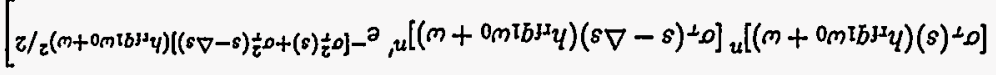

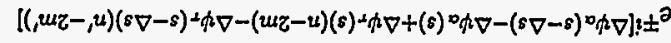

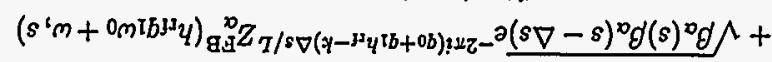

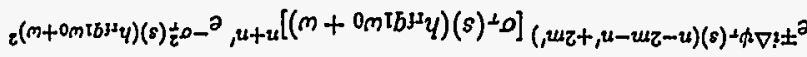

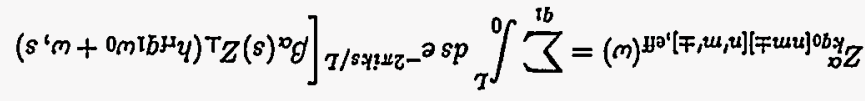




\begin{tabular}{|c|c|c|}
\hline betatron tune & $\nu_{y}$ & 36.64 \\
synchrotron tune & $\nu_{s}$ & 0.03362246 \\
bunch length & $\sigma_{\ell}$ & $1 \mathrm{~cm}$ \\
circumference & $L$ & $2199.318 \mathrm{~m}$ \\
average $\beta$-function & $\left\langle\beta_{y}\right\rangle$ & $18.3647 \mathrm{~m}$ \\
average $\beta$-function at cavities & $\left\langle\beta_{y}\right\rangle_{\text {cav }}$ & $16.8500 \mathrm{~m}$ \\
average $\beta$-function at BPM's & $\left\langle\beta_{y}\right\rangle_{\text {bpm }}$ & $16.6291 \mathrm{~m}$ \\
average $\beta$-function in straights & $\left\langle\beta_{y}\right\rangle_{\text {str }}$ & $22.1851 \mathrm{~m}$ \\
length of straights & $L_{\text {str }}$ & $770.11 \mathrm{~m}$ \\
average $\beta$-function in arcs & $\left\langle\beta_{y}\right\rangle_{\text {arc }}$ & $16.3061 \mathrm{~m}$ \\
length of arcs & $L_{\text {arc }}$ & $1429.208 \mathrm{~m}$ \\
energy & $E$ & $2.5 \mathrm{GeV}$ \\
operating current & $I$ & $3 \mathrm{~A}$ \\
number of cavities & $N_{\text {cav }}$ & 8 \\
rf frequency & $f_{\mathrm{rf}}$ & $476 \mathrm{MHz}$ \\
rf harmonic number & $h_{\mathrm{rf}}$ & 3492 \\
Buckets in symmetric fill & $M$ & 1746 \\
number of bunches & $k_{B}$ & 1658 \\
momentum compaction & $\eta C$ & $1.23204608115 \times 10^{-3}$ \\
horizontal emittance & $\epsilon_{x}$ & $64.43 \mathrm{~nm}$-rad \\
vertical emittance & $\epsilon_{y}$ & $2.57 \mathrm{~nm}-\mathrm{rad}$ \\
vertical damping time & $\tau_{y}$ & $0.0576 \mathrm{~s}$ \\
Energy loss per turn & $\Delta E$ & $0.789988 \mathrm{MeV}$ \\
Maximum rf voltage & $\hat{V}$ & $5.1 \mathrm{MV}$ \\
\hline \multicolumn{2}{|r|}{}
\end{tabular}

Table 5.1: Parameters for the PEP-II low energy ring [10,49]. Note that the energy, current, and number of cavities are worst-case values. The average $\beta$-functions at BPM's and cavities are obtained by averaging the $\beta$-functions at the BPM's and cavities respectively. The average $\beta$-function in the arcs and straights was obtained by using a trapezoid rule integration for the values of the $\beta$-function at the exit face of each element of the respective regions. The arcs are taken to extend from the end of the element prior to the first arc bend in each arc section to the end of the last arc bend in each arc section.

radiation damping rate, and so one would expect some Landau damping. It turns out that there probably is none; this will be discussed in more detail at the end of this chapter.

The effects of chromaticity can be computed from the formalism presented in this dissertation. It is a complex computation, and has not been completed. However, a good estimate of its effect can be obtained by doing what most authors have done $[11,38,40,57-$ $59,63,66,67,70]$, which is to perform the computations by shifting the form factors in (4.84) by $\omega_{\xi}=\xi \omega_{0} / \eta_{C}$, the chromatic frequency. Here $\xi$ is the chromaticity [68]. This will be discussed in more detail at the end of this chapter.

Potential-well distortion can also give effects similar to a nonlinear lattice. In addition to that, however, it can also give purely linear effects. It can cause the tunes and the bunch length to change as the current is increased. These effects can both be taken into account simply by making the linear tune $\nu_{s}$ and the bunch length $\sigma_{\ell}$ in formulas (4.83)-(4.85) functions of current. This is not done here, since the potential-well distortion effects are unknown.

Little potential-well distortion is expected from the vertical transverse wake. The reason is that the lattice typically has "midplane symmetry." This means that the lattice Hamiltonian is purely a function of $y^{2}$ and $p_{y}^{2}$. Thus, the unperturbed distribution will be an even function of $y$, and therefore the wake force due to the unperturbed distribution will be zero (see equations (3.57) and (3.58)).

All of the bunches are assumed to be identical and equally spaced. This ignores the cross terms between different $p_{0}$ and $q_{0}$ from equation (4.83). A simple extension of Kohaupt's [39] work demonstrates that if the current in symmetrically placed bunches is set equal to the current of the highest-current bunch in the real system, then the growth rate for all the modes with the same zero-current frequency in the real system will be bounded by the largest growth rate for the symmetric system. The proof is as follows: from the discussion at the end of section 4.2 , it is clear that equation (4.83) can take the form

$$
\lambda D_{n b}=N_{n} \sum_{r} A_{n b, r a} D_{r a}
$$

after a simple change of basis. The $\Omega$ dependence in $A$ has been ignored for the purpose of this argument; this is an excellent approximation as long as there are no resonances whose width is comparable to or less than $\nu_{s}$ (which is usually the case in the transverse plane). This matrix equation can be formulated as $\lambda D=N A D$. Then assuming that $A$ can be diagonalized, it immediately follows that $|\lambda| \leq \max _{r} N_{r} \max _{n}\left|\lambda_{n}\right|_{2}$ where $\lambda_{n}$ are the eigenvalues of $A$. The eigenvalues of $A$ give the growth rates for the symmetric system. Thus, the symmetric case bounds the multibunch growth rates. Also, the threshold for single-bunch mode coupling depends only on the single-bunch current. Thus, one would expect that for multibunch mode coupling, where single-bunch mode coupling and multibunch modes are in some sense combined, the worst-case results would be obtained by symmetrically filling the bunch buckets with currents which are equal to the current of the highest-current bunch. 
When the bunches are non-symmetric, the tunes of different bunches will often be different, since they see different wakefields and thus have a different potential-well distortion [11,14,46-48]. Thus, for example, one can get Landau damping due to the bunch-tobunch tune spread [14]. The bulk of the bunches have very similar synchrotron frequencies; the variation is about $0.4 \%$. A few bunches at the head and the tail of the bunch train have significantly larger variations (as large as $2 \%$ ) [33]. Since this is only a few bunches, the $0.4 \%$ will probably dominate the Landau damping. This gives a tune spread of about $1.3 \times 10^{-4}$. While this is significantly larger than radiation damping, it will probably not result in any Landau damping, for the reasons discussed later in this chapter.

The multibunch longitudinal potential well distortion will shift the synchrotron frequency as well. The effect gives approximately a $2 \%$ error in the average synchrotron frequency [33].

The terms coupling modes near $\omega_{y}$ to modes near $-\omega_{y}$ (i.e., equation (4.85)) are ignored, introducing errors of order $\nu_{s} / \nu_{y}$, as described at the end of section 4.3.2. These errors are of order $0.09 \%$ for this example.

Only the $k=0$ terms in the effective impedance (4.84) are considered. This approximation amounts to ignoring "synchro-betatron modes"; these are discussed in greater detail in $[9,12,55,64]$. Suzuki's work [64] seems to indicate that this should be a minimal problem with realistic impedances.

$\Delta \psi_{\tau}(s)$ is taken to be zero. This could introduce relative errors of order $2 \pi \nu_{s}$, or about $20 \%$. The errors will probably be significantly smaller than that, since $\psi_{\tau}(s)$ should be (very nearly) monotonically increasing to $\mu_{\tau} . \psi_{\tau}(s)$ only increases in the arc sections, however. Thus, if most of the phase advance takes place in the arc sections (it only takes place there-see equations (4.31) and (A.19) ), of which there are $N_{\mathrm{arc}}=6$, and the dispersion and magnetic field are the same for every bend (a good assumption for this lattice), then the largest $\Delta \psi_{\tau}(s)$ will be around $\pi \nu_{s} L_{\text {str }} / N_{\text {arc }} L$, or about $0.62 \%$. Due to non-uniformity of the phase advance, the real error will be larger. It turns out to be, at most, about $1.9 \%$ for this case, as shown in figure 5.1. Note that this number will get multiplied by the $m$ for the mode (thus, the $m=2$ modes have an error more like $3.8 \%$ ). One can assume that the effect will be even smaller, due to cancelling of oscillations. The average of the data in figure 5.1 is about 0.007 , giving a reduction of about a factor of 3 . However, if significant parts of the impedance are at a particularly bad point, the worst case value may be more appropriate. Note that this error does not enter for $m=0$ modes (including radial modes).

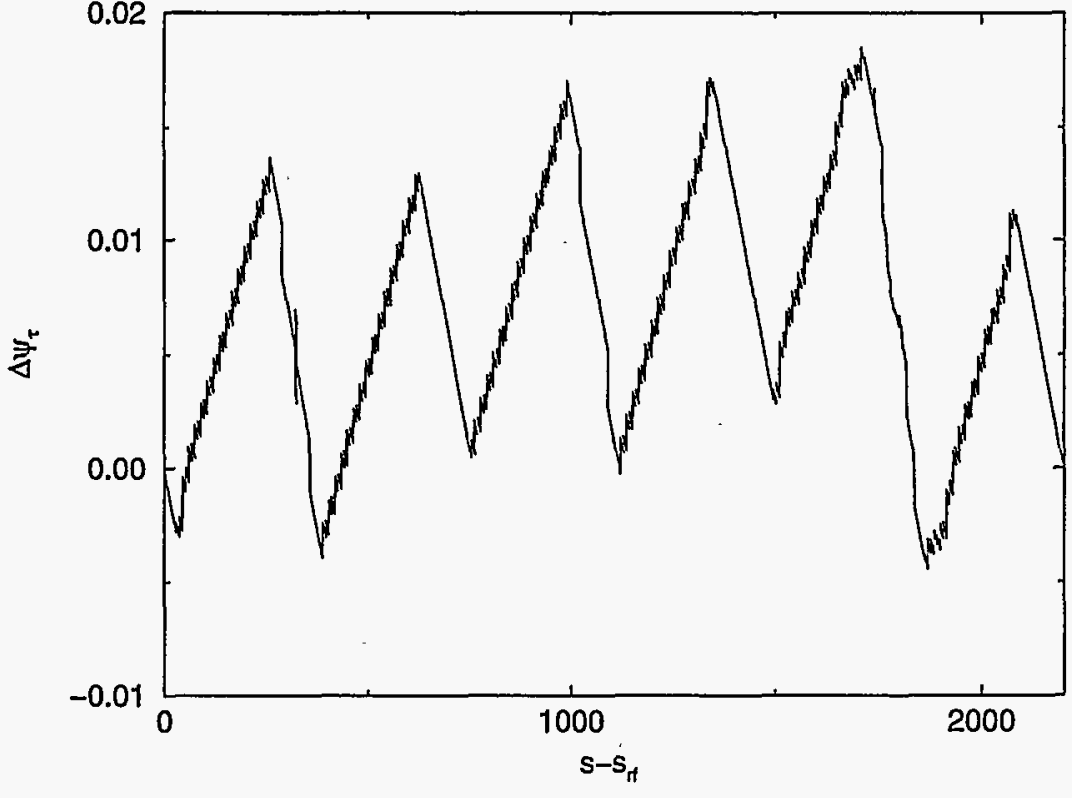

Figure 5.1: $\Delta \psi_{\tau}(s)$, plotted versus position in the ring. Data from Yunhai Cai [10].

$\sigma_{\tau}(s)$ is taken to be constant. From equation (A.29), this leads to an error of approximately $1 \%$.

Altogether, these errors indicate that this computation will be good to a few percent. In reality, the error is probably not dominated by these errors, but by how well the impedance is known.

All growth rates can be compared to the damping rate due to synchrotron radiation [62]. The beam will be considered stable if all growth rates are smaller than this radiation damping rate.

\subsection{Impedance Model}

There are several sources of transverse impedance included in this calculation. Each will be described in detail below. 
Because of the approximations described in section 5.1, to compute the effective impedance (4.84), the only lattice parameters one needs to find for each impedance are the average $\beta$-functions over the region where the impedance is in the ring.

\subsubsection{Resistive Wall}

The resistive wall impedance is given by the formula

$$
z_{\perp}^{\mathrm{RW}}(\omega)=-i \sqrt{2} \frac{R_{\mathrm{RW}}}{\sqrt{-i \omega / \omega_{0}}}
$$

where $R_{\mathrm{RW}}$ is given by

$$
R_{\mathrm{RW}}=F \frac{Z_{0} L_{p}}{2 \pi b_{p}^{3}} \sqrt{\frac{2 c}{\omega_{0} \sigma Z_{0}}}=F \frac{Z_{0} L_{p}}{2 \pi b_{p}^{3}} \sqrt{\frac{c^{2}}{2 \pi \omega_{0} \sigma}} .
$$

Here $b_{p}$ is the smallest half-dimension (vertical or horizontal) of the vacuum chamber, $L_{p}$ is the length of the vacuum chamber, $\sigma$ is the vacuum chamber wall conductivity, $Z_{0}$ is the impedance of free space $(\approx 377 \Omega)$, and $F$ is a form factor which depends only on the shape of the vacuum chamber [28].

The arc sections of the vacuum chamber are elliptical with a major axis of 3.740 inches and a minor axis of 2.170 inches [50]. This geometry makes $F 0.8263$ [28]. The vacuum chamber is made of $6063 \mathrm{~T} 5$ aluminum alloy [50], which has a resistivity of $1 / \sigma=32 \Omega$ $\mathrm{nm}$ [4]. Thus, $R_{\mathrm{RW}}$ for the arc sections is $825 \mathrm{k} \Omega / \mathrm{m}$.

The straight sections of the vacuum chamber are round with a diameter of $3.5 \mathrm{in}$. [51]. $F$ is 1 for this geometry [28]. The vacuum chamber is made of 304 stainless steel [35], giving an resistivity of $1 / \sigma=720 \Omega$-nm [4]. Thus, $R_{\mathrm{RW}}$ for the straight sections is $608 \mathrm{k} \Omega / \mathrm{m}$.

\subsubsection{Inductive}

Many devices, such as bellows, BPM's, and slots, give an impedance that is primarily inductive. The inductive part of the transverse impedance is obtained by scaling the longitudinal inductive impedance of $83.3 \mathrm{nH}$ [31] by $2 c / \omega b_{p}^{2}$ [11], where $b_{p}$ is a characteristic dimension of the beam pipe. Worst-case values are obtained by performing this scaling with $b_{p}=2.7559 \mathrm{~cm}$, the vertical size of the arc section chamber [50].

The impedance will not be constant for all frequencies; it is expected to begin to roll off at high frequencies. Since the average behavior at high frequencies is expected to be similar to that of a cavity, a high-frequency roll-off of $\omega^{-3 / 2}$ is assumed [33]. The form for the transverse impedance is thus

$$
z_{\perp}^{\text {Ind }}(\omega)=\frac{-i R_{\text {Ind }}}{\left(1-i \omega / \omega_{C}\right)^{3 / 2}}
$$

where $R_{\text {Ind }}$ is the transverse impedance associated with the inductance. To determine the cutoff frequency $\omega_{C}$, the loss factor from $Z_{\perp}^{\text {Ind }}$ is computed as a function of $\omega_{C}$, and compared to the total loss factor from inductive components of $3.4 \mathrm{~V} / \mathrm{pC}$ [31]. The total loss factor is

$$
-i \frac{L}{2 \pi} \int_{-\infty}^{\infty} e^{-\omega^{2} \sigma_{\tau}^{2}} \frac{\omega d \omega}{\left(1-i \omega / \omega_{C}\right)^{-3 / 2}}
$$

where $L$ is the inductance (a Gaussian bunch has been assumed). Using the bunch length of $1 \mathrm{~cm}$, this formula gives the right loss factor when $\omega_{C} \approx 19.8 \mathrm{GHz}$. Note that $R_{\mathrm{Ind}}=2 c L / b_{p}^{2}$.

\subsubsection{Cavity Tails}

It is well known that the longitudinal impedance of a single cavity rolls off at high frequency as $\omega^{-1 / 2}$ [32]. A simple model with the appropriate high-frequency roll-off is

$$
Z_{\|}^{\text {Tail }}(\omega)=i R_{\text {Tail }}\left[\left(1+\frac{\omega}{\omega_{C}+i \alpha}\right)^{-1 / 2}-\left(1-\frac{\omega}{\omega_{C}-i \alpha}\right)^{-1 / 2}\right]
$$

This model is fit to a model of the cavity run through $\mathrm{ABCI}$ for $m=1[13,31]$ with the known higher order modes removed. The parameters are found to be $R_{\text {Tail }}=45.1344 \mathrm{k} \Omega / \mathrm{m}^{2}$, $\omega_{C}=2.4 \mathrm{GHz}$, and $\alpha=1.34722 \mathrm{GHz}$ [5]. This impedance must of course be multiplied by the number of cavities.

The longitudinal impedance (5.6) is scaled by $\beta_{0} c / \omega$ to obtain the transverse impedance (see section 3.1.4).

\subsubsection{Cavity Higher Order Modes}

A preliminary list of the cavity higher order modes is given in table 5.2. Each mode is considered to be a single resonator of the form [11]

$$
Z_{\perp}^{\text {res }}\left(x \omega_{R}\right)=\frac{R_{\mathrm{res}}}{x+i Q\left(1-x^{2}\right)}
$$

These impedances must of course be multiplied by the number of cavities. 


\begin{tabular}{|c|c|r|}
\hline $\begin{array}{c}\text { Frequency } \omega_{R} \\
\mathrm{GHz}\end{array}$ & $Q$ & $\begin{array}{r}R_{\text {res }} \\
\mathbf{k} \Omega / \mathrm{m}\end{array}$ \\
\hline 0.796 & 115 & 34.916 \\
1.068 & 27 & 16.911 \\
1.127 & 54 & 0.162 \\
1.212 & 871 & 10.135 \\
1.333 & 611 & 58.328 \\
1.413 & 1138 & 160.689 \\
1.542 & 92 & 2.508 \\
1.603 & 145 & 7.777 \\
1.668 & 783 & 153.796 \\
1.738 & 446 & 0.177 \\
1.766 & 1317 & 2.414 \\
\hline
\end{tabular}

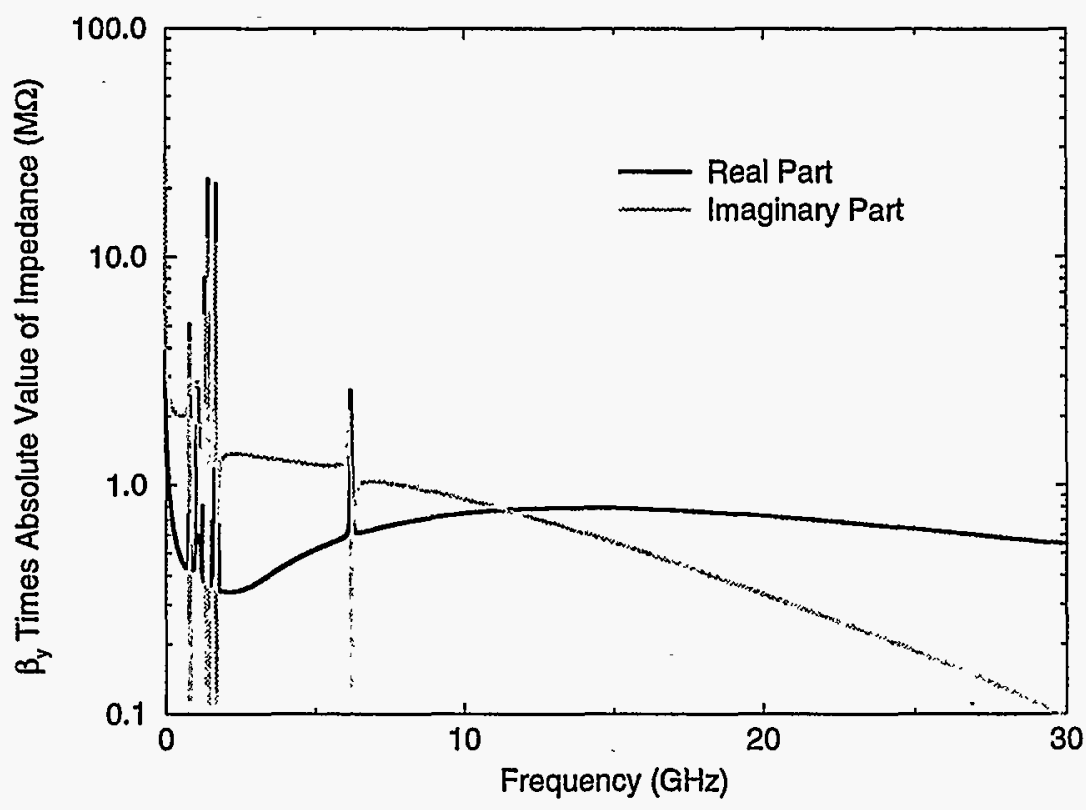

The beam position monitors (BPM's) also have a resonance at $6.2 \mathrm{GHz}$, with a $Q$ of about 200 , and a total $R_{\text {res }}$ for all the BPM's of $120 \mathrm{k} \Omega / \mathrm{m}$.

\subsubsection{Total Impedance}

The total impedance from all these sources, multiplied by the average $\beta$-function at each source, is plotted in figures 5.2 and 5.3. As can be seen from equation (4.84), these $\beta$-function weighted impedances are multiplied by the form factors

$$
\left(\sigma_{\tau} \omega\right)^{n+n^{\prime}} e^{-\sigma_{\tau}^{2} \omega^{2}}
$$

which are plotted in figures 5.4 and 5.5. The form factors show that different modes tend to sample different parts of the impedance, and in fact that modes with larger $m$ tend to sample impedances at higher frequency. But note that the peak of the form factor (5.8) occurs at $\omega \sigma_{\tau}=\sqrt{\left(n+n^{\prime}\right) / 2}$. Thus, the peaks move out fairly slowly as $m$ is increased; if the impedance has strong high-frequency components, it may require a very large number of modes to determine the behavior of the system.

Figure 5.2: Absolute value of the total impedance defined in section 5.2, multiplied by the appropriate $\beta$-functions from table 5.1. The real part is always positive, while the imaginary part changes signs several times, mainly at narrow resonances. The vertical spikes which go down to low values generally indicate such a sign change.

\subsection{Frequencies and Growth Rates}

\subsubsection{Single Bunch}

Begin by considering a single bunch. As can be seen from figures 5.6 and 5.8 , the growth rates are zero until the some of the mode frequencies coincide. At that point, the growth rates increase sharply.

The phenomenology can be understood from analysis of a two mode example. The two-by-two eigenvalue system to be solved involves solving the equation

$$
\operatorname{det}\left[\begin{array}{cc}
\Omega-I Z_{11} & -I Z_{12} \\
-I Z_{21} & \Omega-k \omega_{3}-I Z_{22}
\end{array}\right]=0
$$




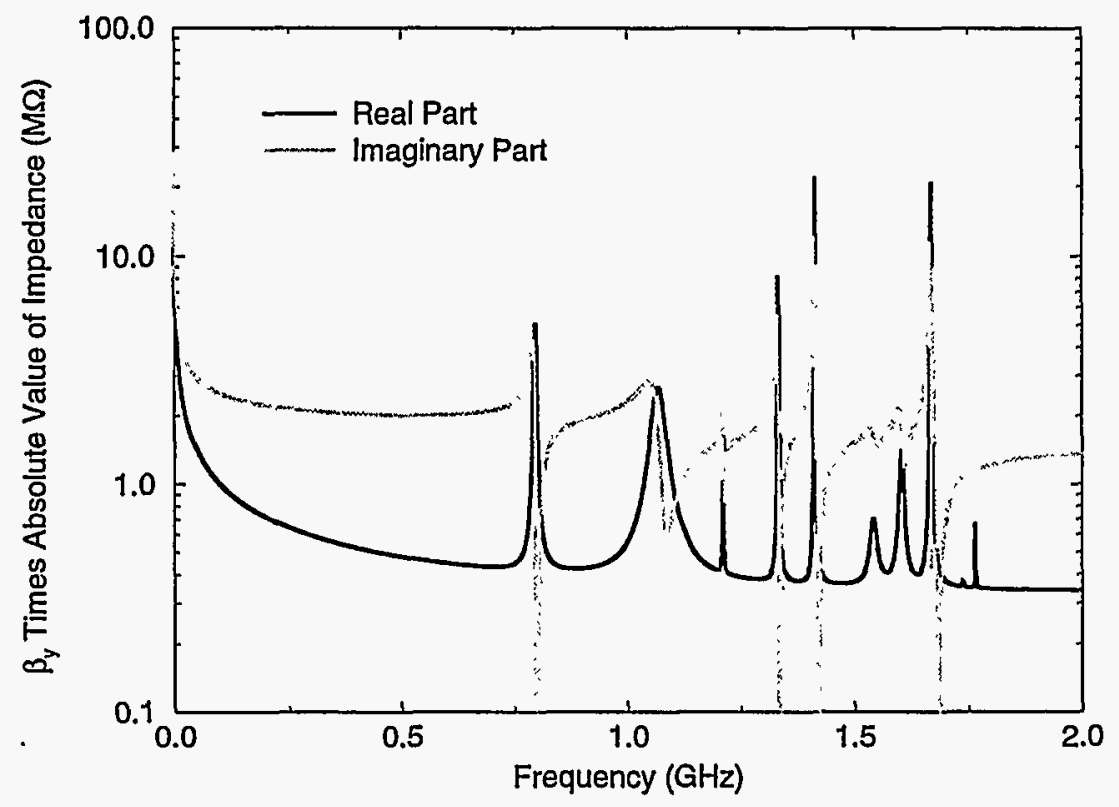

Figure 5.3: Low frequency part of the total impedance plotted in figure 5.2. This shows the cavity higher order modes in more detail.

where $I$ is the total current, and $k \omega_{s}$ is the separation between the mode frequencies at zero current. The $Z$ 's can usually be taken to be sufficiently slowly varying functions of $\Omega$ that they can be regarded as constants.

For a single bunch, the computation of the $Z_{i j}$ can be done most simply by taking $h_{\mathrm{rf}}=1$ ( $\omega_{\mathrm{rf}}$, of course, should keep its physical value). Thus, as long as $Z$ is relatively slowly varying, the summations over $q_{1}$ in (4.84) are very close to being symmetric. Thus, the real part of $Z_{k[m n \pm]\left[m^{\prime} n^{\prime} \pm\right]}^{1}$ dominates the imaginary part when $n+n^{\prime}$ is even, while the the imaginary part dominates when $n+n^{\prime}$ is odd. Therefore, take $Z_{11}$ and $Z_{22}$ to be real, and $Z_{12}$ and $Z_{21}$ to be imaginary when $k$ is odd. For $k$ even, $Z_{12}$ and $Z_{21}$ become real. Also note that $Z_{12}$ and $Z_{21}$ will always have the same sign (one is simply a multiple of the other).

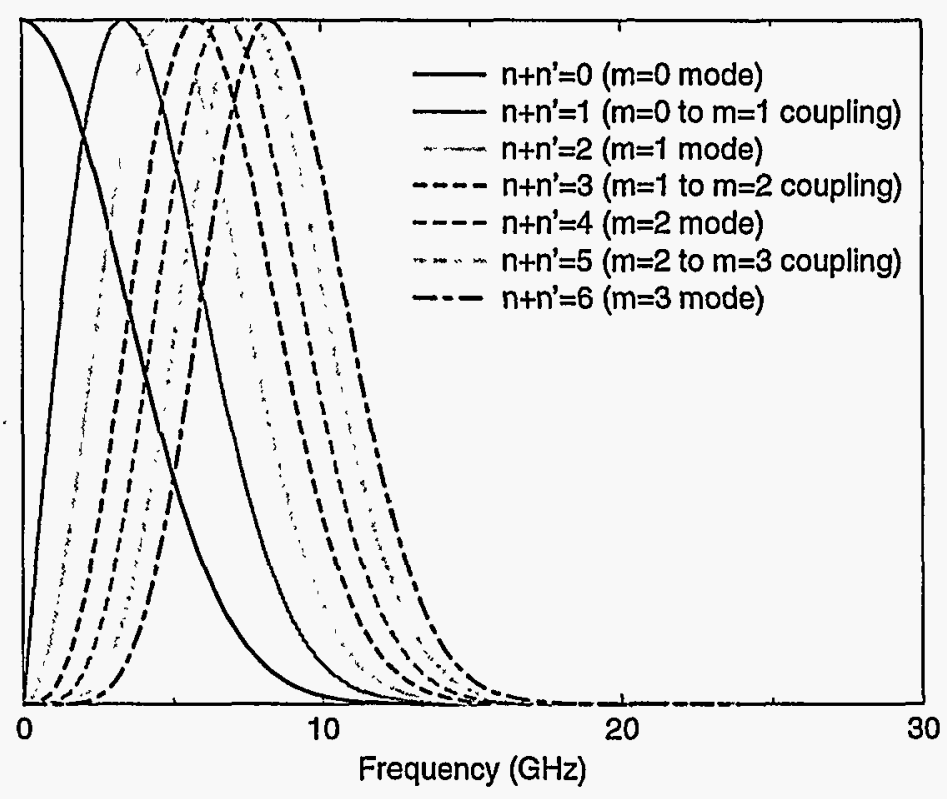

Figure 5.4: Form factors from equation (5.8), normalized to all have the same peak value. The descriptions for each line indicate the mode or coupling terms over which they probably have the strongest influence.

\section{Equation (5.9) leads to the polynomial equation}

$$
\Omega^{2}-\left(I Z_{11}+I Z_{22}+k \omega_{s}\right) \Omega+I^{2}\left(Z_{11} Z_{22}-Z_{21} Z_{12}\right)+I Z_{11} k \omega_{s}=0
$$

This has the solutions

$$
\begin{gathered}
\Omega=\frac{1}{2}\left[r T+k w_{s} \pm \sqrt{\left(I D+k \omega_{s}\right)^{2}+I^{2} P}\right] \\
T=Z_{22}+Z_{11} \\
D=Z_{22}-Z_{11} \\
P=4 Z_{21} Z_{12} .
\end{gathered}
$$

Note that $P$ is negative when $k$ is odd, and $P$ is positive when $k$ is even. Thus, for low currents, the modes are clearly pure real. If $k$ is even, the argument of the square root in 


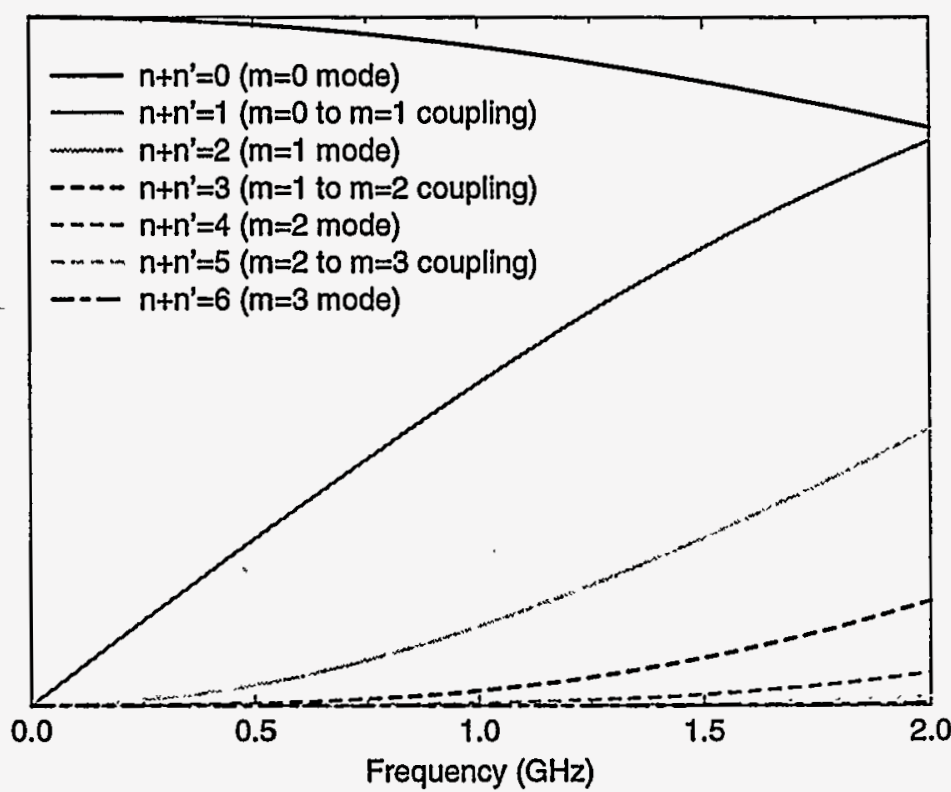

Figure 5.5: Form factors of figure 5.4 over frequency range matching figure 5.3.

(5.11) will remain positive. Thus, there will be no instability due to mode coupling between such modes. Such an "avoided crossing" is shown in figure 5.9.

If $k$ is odd, however, the situation becomes more interesting, since $P$ is negative. If $D$ and $k$ have opposite signs, or if $-P>D^{2}$, then eventually the argument of the square root in (5.11) will become zero. At that point, the mode frequencies coincide. As the current is increased from that point, the square root becomes negative, and thus $\Omega$ gets an imaginary part. Since one of the imaginary parts must be positive, the beam is unstable. If $-P>D^{2}$, $\Omega$ will have an imaginary part for all higher currents. The growth rate will asymptotically be $I \sqrt{-P-D^{2}}$.

However, if $-P<D^{2}$, then eventually, the current will increase to the point where the argument of the square root again becomes zero. At this point, both mode frequencies will be real again. Thus, in a plot of the growth rates versus current, there will be a "bubble"

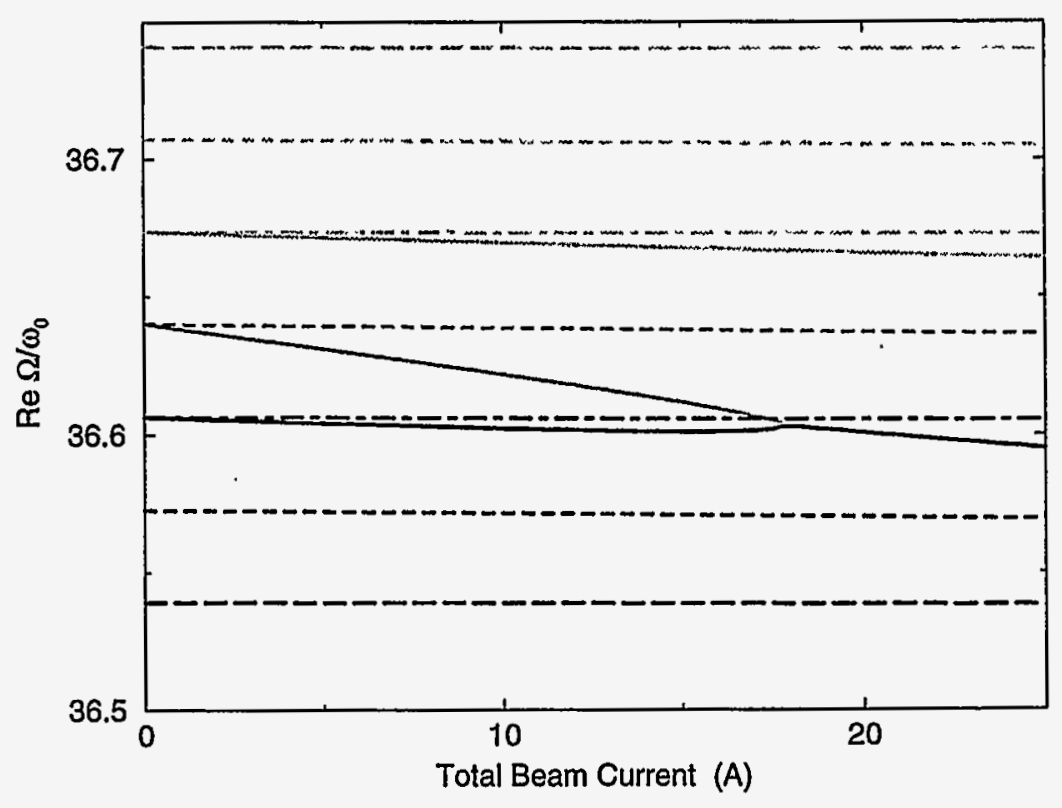

Figure 5.6: Single-bunch mode frequencies, plotted versus total beam current for 1746 bunches. Frequencies shift with increasing current until two frequencies coincide. The frequencies continue to coincide for higher currents. Note that the line style and shade of grey for each mode in this figure will be used for the corresponding modes in future figures. Line types are identified in figure 5.7.

for the range of currents where the square root was negative, and the growth rate will be zero for currents above and below this bubble. A small bubble indicates that there was very little coupling between the two modes in question. This can be seen in figure 5.8: there is very weak coupling between the $m=0$ mode and the $m=1$ radial mode; thus, there is only a nonzero growth rate over a small range of currents. However, when the $m=0$ mode couples to the main $m=1$ mode, there is much stronger coupling, and the growth rates continue to increase for very high currents. Note, however, that the growth rates due to the small bubble are nontrivial. Thus, the threshold of instability is really the beginning of the small bubble. 


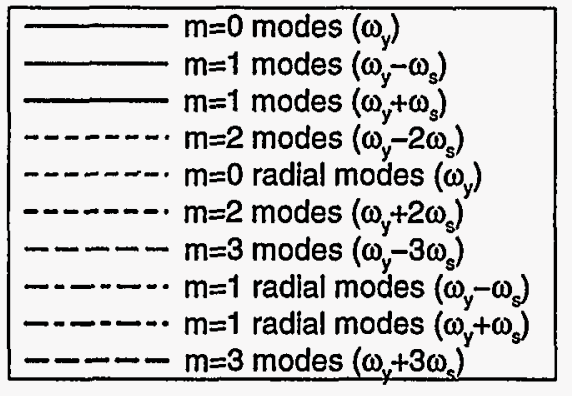

Figure 5.7: Legend describing line types used in mode plots.

As can be seen from figure 5.6, one can obtain a good estimate of the instability threshold current due to mode coupling by assuming that the $m=0$ mode frequency shift increases linearly with current, and that the instability occurs once it has shifted by $-\omega_{3}$. This condition, combined with equations (4.83) and (4.84), gives an estimate of the single-bunch mode coupling threshold current of

$$
4 \pi \beta_{0}^{2} \frac{E_{0}}{e}\left|\frac{\sigma_{\tau} \omega_{0} \nu_{s}}{\int d s d x \beta_{y}(s) \Im\left\{Z_{\perp}\left(x / \sigma_{\tau}, s\right)\right\} e^{-x^{2}}}\right| \text {. }
$$

If the primary contribution to the broadband impedance is assumed to come from a pure inductance $-i R_{\text {Ind }}$ plus the resistive wall, then the denominator in (5.15) is just

$$
-\left\langle\beta_{y}\right\rangle R_{\mathrm{Ind}} \sqrt{\pi}+\frac{\sqrt{\sigma \omega_{0}}}{2} \Gamma(1 / 4) \sum_{k}\left\langle\beta_{y}\right\rangle_{k} R_{\mathrm{RW}, k} .
$$

Putting in the inductive and resistive wall values calculated above, the threshold curren comes out to be $21.9 \mathrm{~A}$. Comparing to figure 5.6, this is somewhat higher than the actual threshold. This is because the estimate of the threshold current doesn't include all contributions, and the fact that the model assumed isn't quite accurate. It does, however, give a reasonable first estimate. Similar computations have been suggested by other authors $[57,63,71]$.

\subsubsection{Multibunch Mode Frequencies}

Now, consider what happens to the mode frequencies from figure 5.6 when there are multiple bunches. If coupling between the multibunch modes is ignored, the frequencies behave as

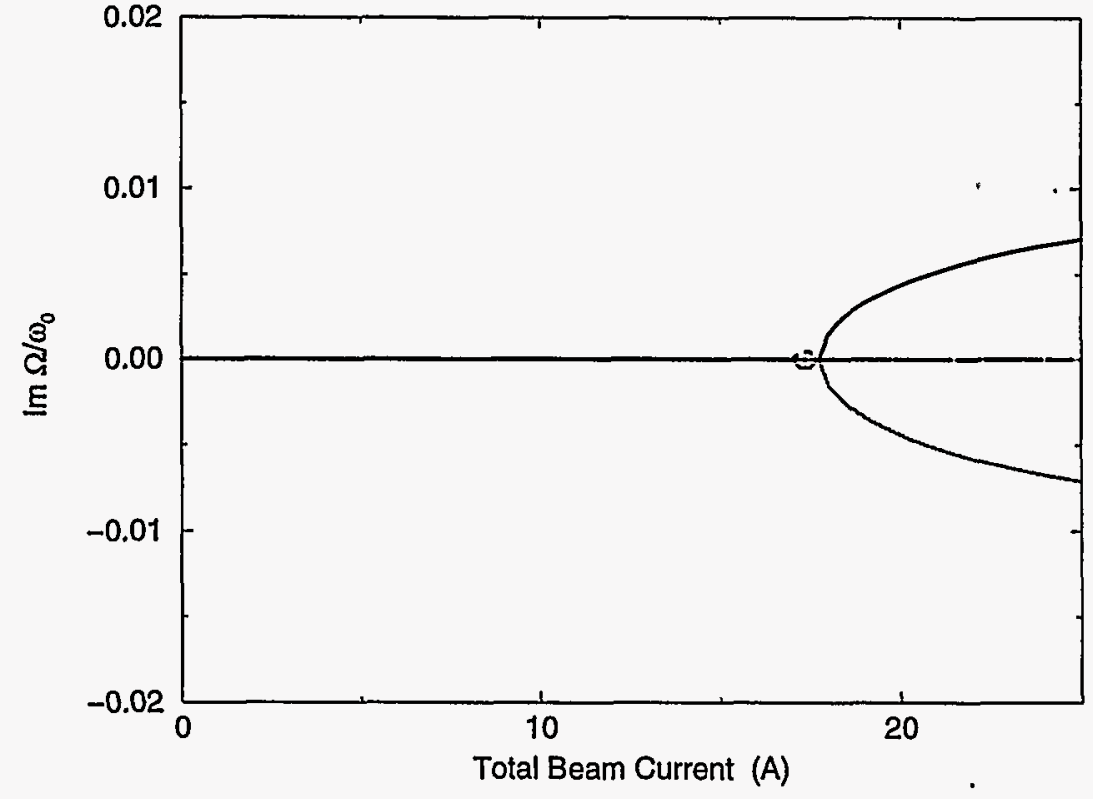

Figure 5.8: Single-bunch growth rates, plotted versus total beam current for 1746 bunches. Growth rates are zero until the frequencies of two modes coincide (see figure 5.6). The growth rates then have a nonzero value for higher currents.

shown in figure 5.10. The mode frequencies are a linear function of current, as can be seen from equation (4.83) (ignoring the dependence of $Z_{k[n m t]}^{\frac{1}{1}}\left[n^{\prime} m^{\prime} \pm\right]$,eff on $\omega$, at least over the range of frequency shifts). Note that, at least for low currents, the average behavior of the multibunch modes is just that of the single bunch modes. Also, notice that the frequencies of the multibunch modes coincide at significantly lower currents (about $10 \mathrm{~A}$ ) than the single-bunch modes did (about $17 \mathrm{~A}$-see figure 5.6). Thus, for some of the multibunch modes, one expects to see increased growth rates due to mode coupling beginning at around $10 \mathrm{~A}$.

The frequency shifts seem to change minimally when coupling is introduced, as shown in figure 5.11. The frequencies at which the modes coincide do not seem to have changed much. Many of the multibunch mode "fans" have broadened slightly, particularly the $m=1$ 


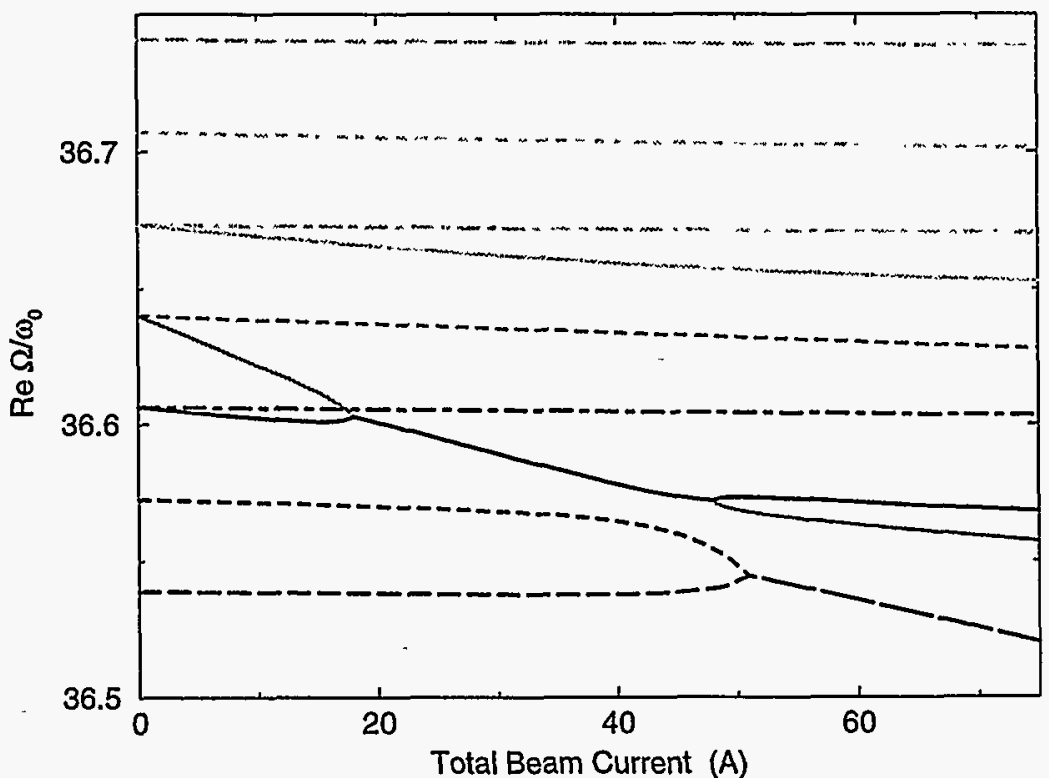

Figure 5.9: Frequencies due to single bunch mode coupling as in figure 5.6, but taken at higher currents. Note the avoided crossing between the $m=0$ mode and the $m=2$ mode.

ones. Most of the interesting effects will be seen upon examining the growth rates.

An estimate of the threshold for mode coupling effects can be made analogously to that for single-bunch mode coupling. Since narrow-band impedances contribute to the deviation of the impedance from the broadband average, one simply divides the denominator of (5.15) by the number of bunches, then adds to the resulting denominator a term for the largest narrow-band impedance, which would be

$$
\omega_{0} \sigma_{\tau} \Im\left\{Z_{\perp}^{\text {peak }}\right\} \beta_{y} e^{-\sigma_{\tau}^{2} \omega_{\text {peak }}^{2}}
$$

So, begin by computing what the denominator of (5.15) would be given that the threshold current is $17 \mathrm{~A}$ for 1746 bunches (see figures 5.6 and 5.8 ); the result is $1775 \Omega$. Examining figure 5.2 , the resistive wall peak near zero frequency gives the largest peak. That peak is

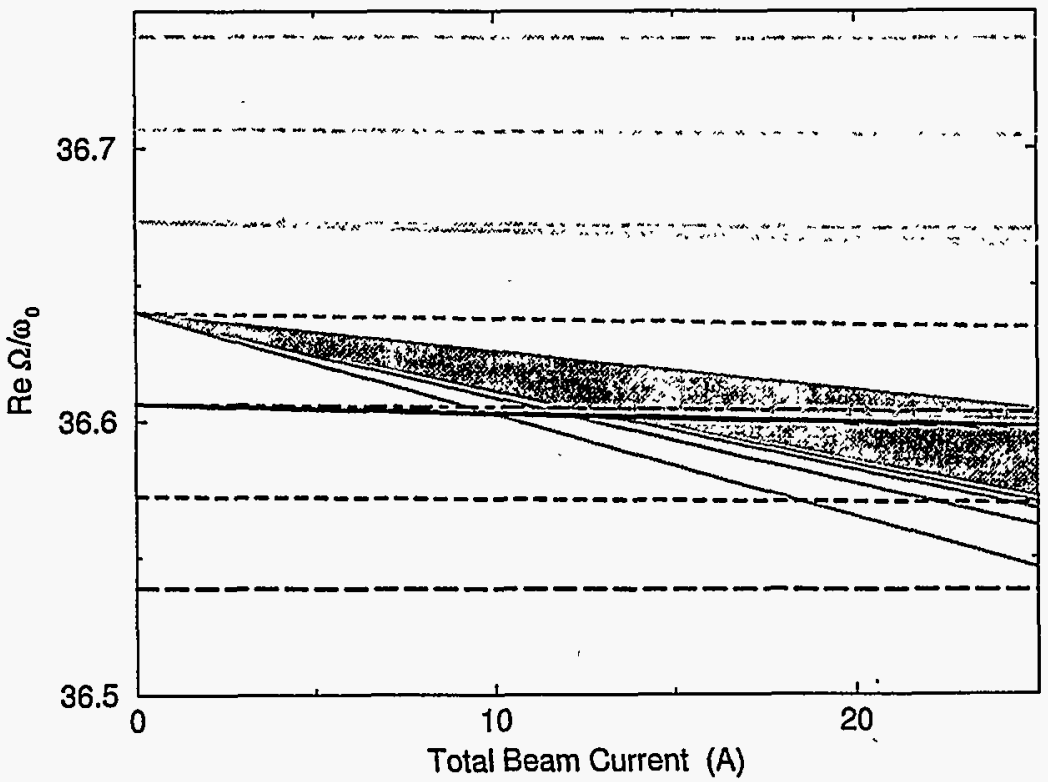

Figure 5.10: Multibunch mode frequencies, without coupling, plotted versus total beam current. Each fan consists of 1746 lines, which correspond to the 1746 multibunch modes.

$$
-\frac{R_{\mathrm{RW}}}{\sqrt{\left\lceil\nu_{y}\right\rceil-\nu_{y}}}
$$

for the imaginary part, which gives a contribution of $1283 \Omega$ for. this system. Adding this to the contribution from the broadband, the threshold current becomes $9.9 \mathrm{~A}$, which is an excellent guess, as can be seen from figure 5.11.

\subsection{3 $m=0$ Growth Rates}

The growth rates for the $m=0$ modes without coupling are shown in figure 5.12. Note that the largest growth rates are large compared to the growth rates due to single-bunch mode coupling, as shown in figure 5.8. However, many of the modes (those which don't 


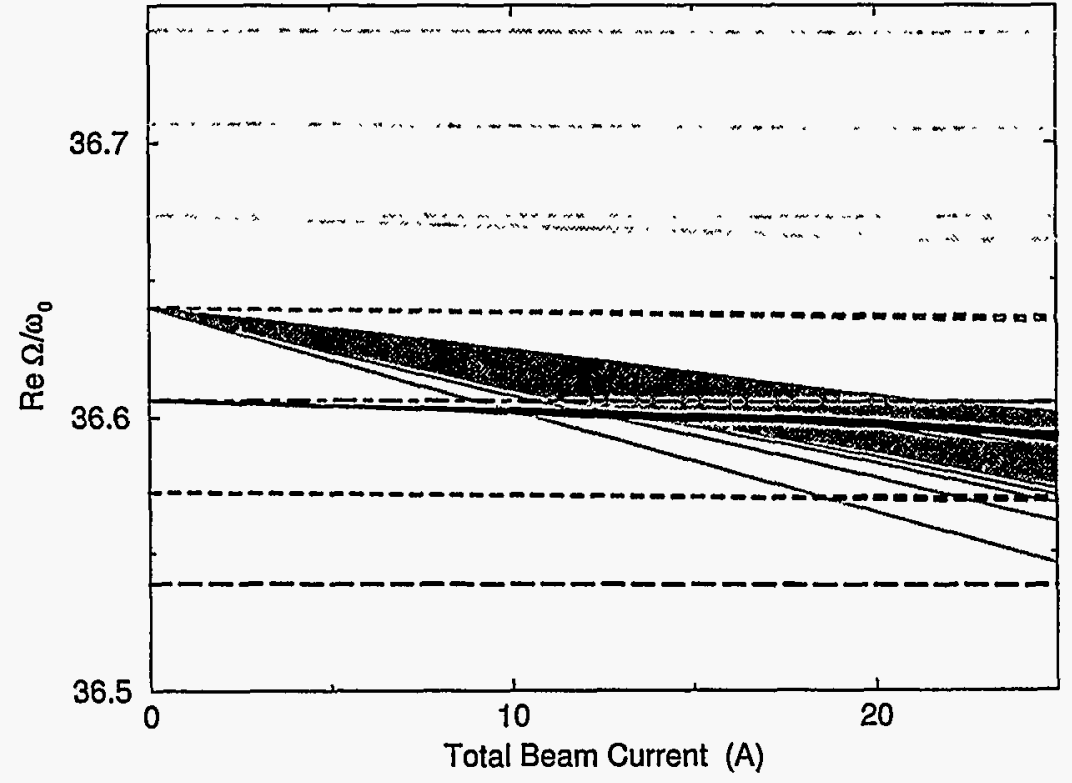

Figure 5.11: Multibunch mode frequencies with coupling, plotted versus total beam current.

overlap with any narrow-band impedances) have negligible growth rates; once coupling is introduced, one might expect those modes to behave similarly to the modes for a single bunch.

Once coupling between the multibunch modes is introduced, the growth rates are those shown in figure 5.13. The modes which had the largest growth rates in figure 5.12 are only slightly affected when mode coupling is introduced. This is probably because the growth rates from single-bunch mode coupling are small (see figure 5.8) compared to the multibunch growth rates, and therefore the multibunch growth rates aren't changed much. Thus, multibunch mode coupling is not expected to have a significantly adverse effect on the $m=0$ modes.

However, the modes with small growth rates in figure 5.12 essentially mirror the singlebunch behavior of figure 5.8 , as can be seen in figure 5.13. This is because those multibunch

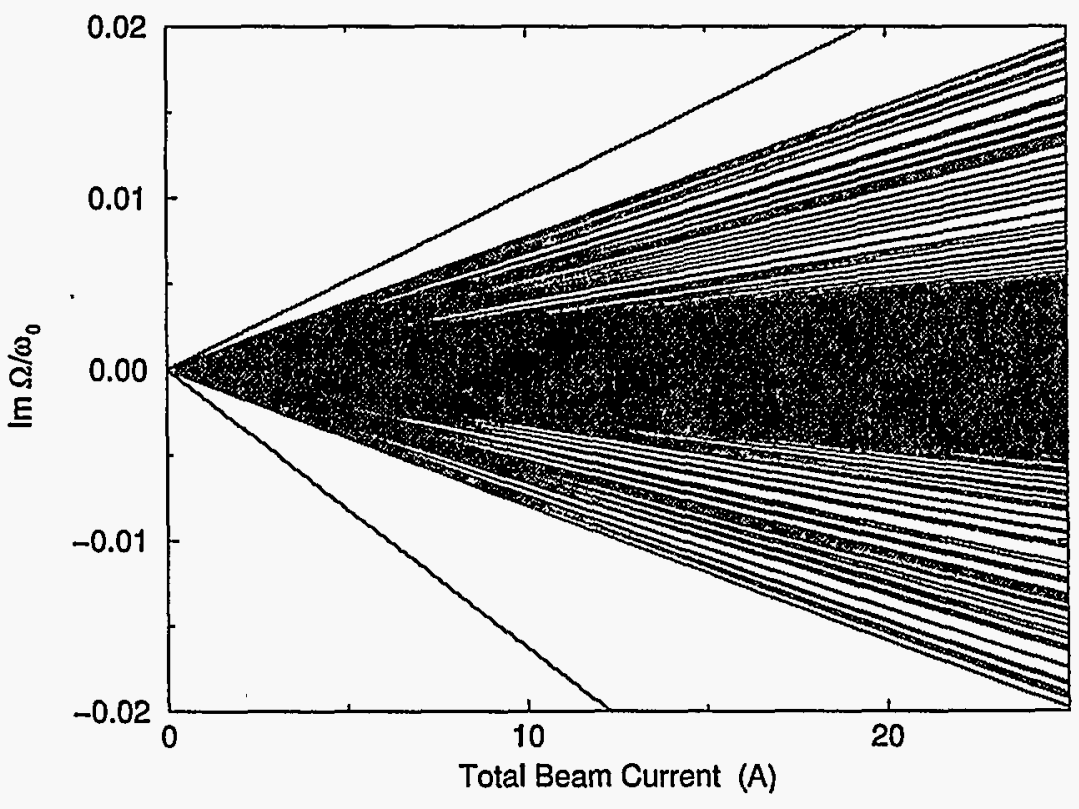

Figure 5.12: $m=0$ mode growth rates, without coupling between multibunch modes. The $1746 m=0$ multibunch modes are each plotted versus current.

modes are driven by wakefields that couple one bunch to the next only very weakly; thus, the effects that are seen are essentially single bunch effects.

\subsection{4 $m=1$ Growth Rates}

The growth rates for the $m=1$ multibunch modes when coupling is ignored is shown in figure 5.14. Note that the growth rates increase linearly with current. Also, note that the largest growth rates are significantly smaller than the growth rates due to single-bunch mode coupling shown in figure 5.8. Thus, multibunch mode coupling is expected to strongly affect these modes.

The growth rates for the $m=1$ multibunch modes with coupling are shown in figures 5.15 and 5.16. Comparing figure 5.15 to the single-bunch mode coupling growth rates 


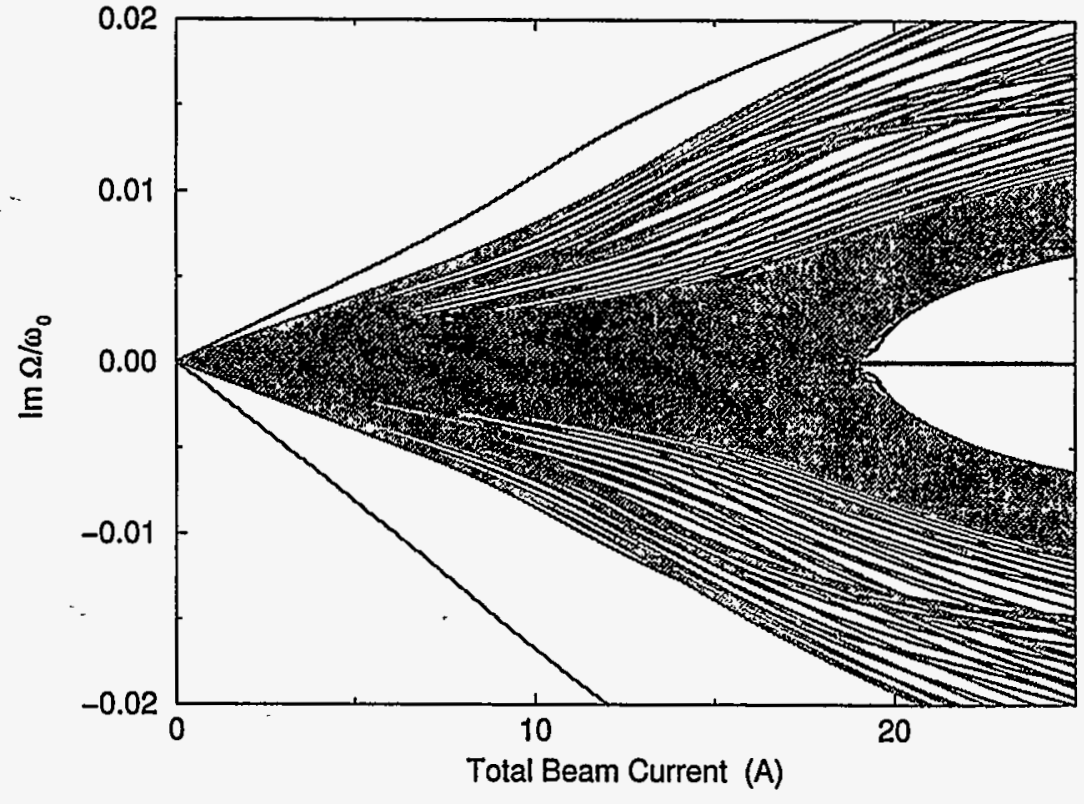

Figure 5.13: $m=0$ mode growth rates, with coupling between multibunch modes.

shown in figure 5.8, it is clear that many of the modes approximately mirror the behavio of single-bunch mode coupling. However, it is also clear from figure 5.15 and especially figure 5.16 that many of the modes have behavior similar to a single-bunch mode coupling threshold, but at currents much lower than the actual single-bunch threshold current. In fact, figure 5.16 shows that the lowest current at which some mode seems to get a sharply increased growth rate is slightly below $10 \mathrm{~A}$, which is the current where the multibunch mode frequencies coincided in figure 5.11. Thus, the $m=1$ modes are very strongly effected by multibunch mode coupling.

Comparing figure 5.16 to figure 5.14, the group of $m=1$ modes with frequencies near $\omega_{y}+\omega_{s}$ (see figure 5.11) are nearly unaffected by mode coupling; this is expected, since the $m=0$ mode frequencies do not approach this group of modes.

Finaily, look at the $m=1$ modes at low currents in more detail, as shown in figure

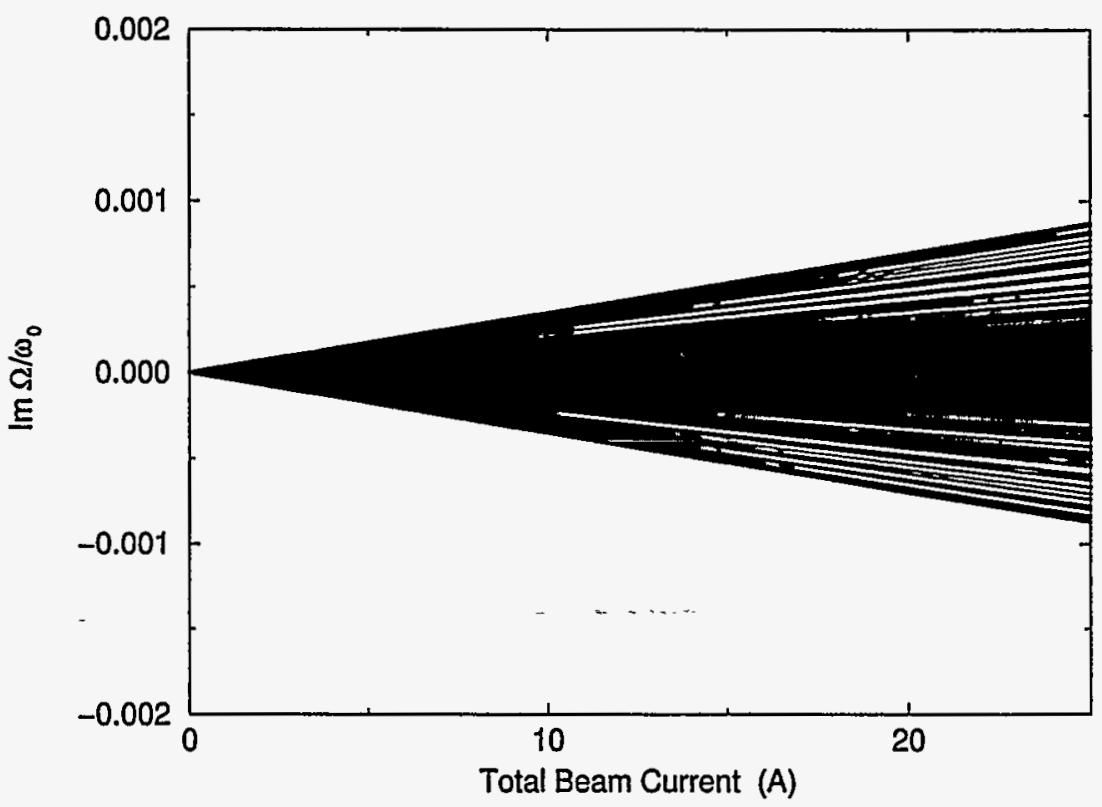

Figure 5.14: $m=1$ multibunch mode growth rates, without coupling.

5.17. Note that even for currents well below the point where the mode frequencies coincide (compare figure 5.11), the growth rates are changing very nonlinearly with current. Since the multibunch modes have nonzero growth rates, their frequency is not sharp, but broadened by the nonzero imaginary part. This causes mode coupling to have an effect even at low currents, as demonstrated in figure 5.17. Thus, mode coupling effects need to be considered even at currents well below the current where the mode frequencies coincide.

\subsection{5 $m=2$ Growth Rates}

The growth rates of the $m=2$ modes (those with frequencies $\omega_{y} \pm 2 \omega_{s}$ ) without coupling between the multibunch modes is shown in figure 5.18. As usual, the growth rates increase linearly with current.

Note that these growth rates are even smaller than the $m=1$ growth rates. As $m$ 


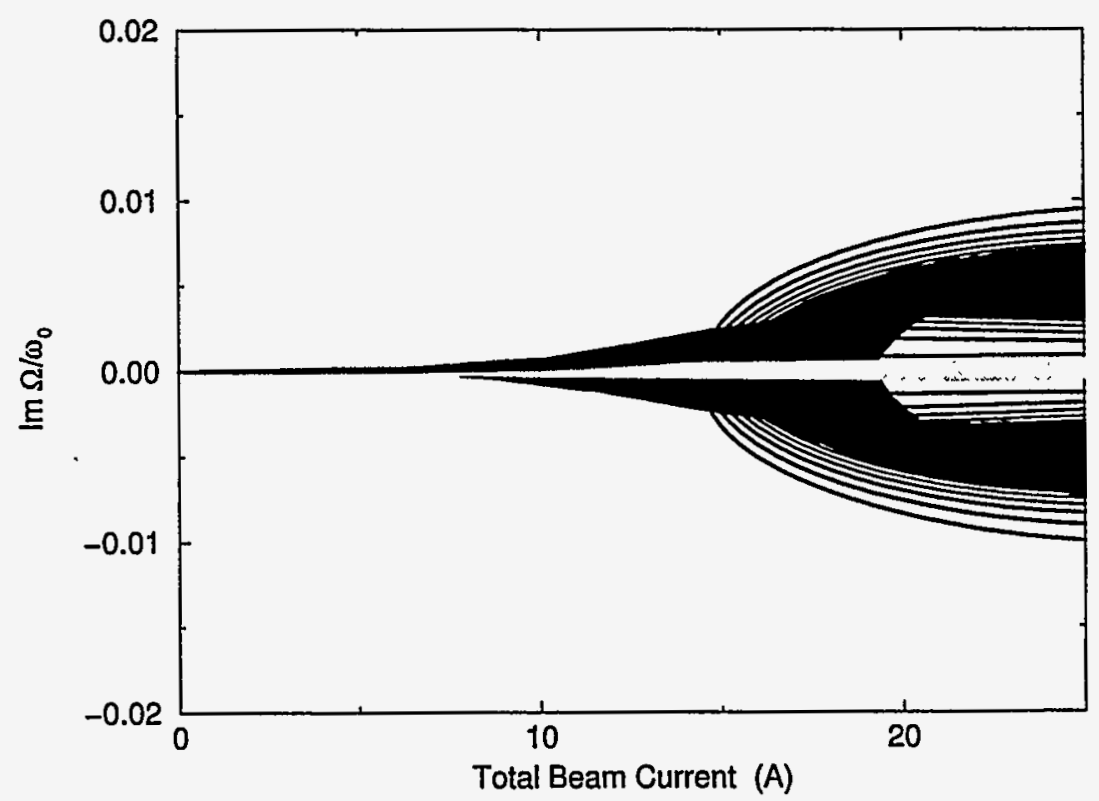

Figure 5.15: $m=1$ multibunch mode growth rates, with coupling.

increases, this is expected to be the trend. The impedance will be multiplied by the form factor

$$
\frac{1}{2^{m} m !}\left(\omega \sigma_{\tau}\right)^{2 m} e^{-\omega^{2} \sigma_{\tau}^{2}}
$$

and integrated over $\omega$. Note that this form factor peaks at $\omega \sigma_{\tau}=\sqrt{m}$, at a value which approaches $e 2^{-m} / \sqrt{2 \pi(m+1)}$ for large $m$. Asymptotically, the half-width of the peaks approaches 0.5 in units of $\omega \sigma_{\tau}$. Thus, the form factor (5.19) picks out higher and higher frequency components as $m$ increases, multiplied by a factor which decreases exponentially with $m$. Since the impedance is not expected to increase exponentially with $m$, and since the width of the peaks is asymptotically constant, the growth rates should decrease rapidly with increasing $m$.

The growth rates of the $m=2$ modes with coupling are shown in figures 5.19 and 5.20. Note that the effects of mode coupling are strong at less than $10 \mathrm{~A}$ (see figure 5.20;

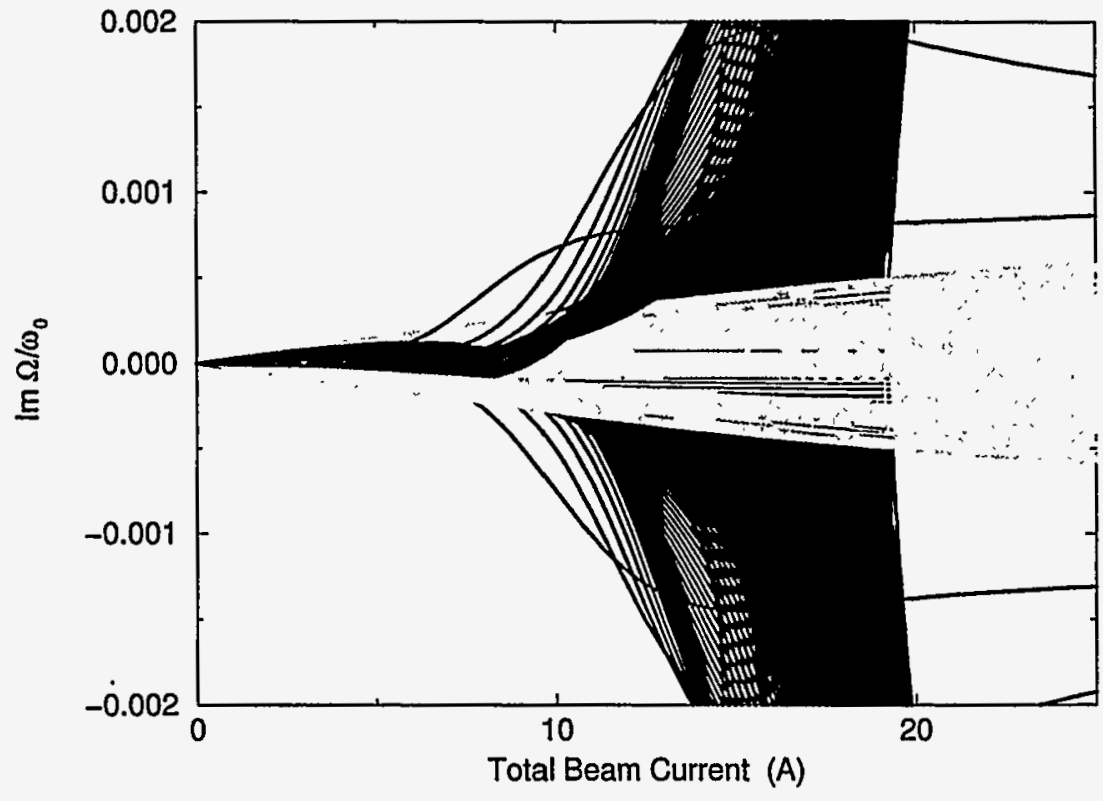

Figure 5.16: $m=1$ multibunch mode growth rates, with coupling. Vertical scale is blown up from figure 5.15

compare figure 5.18), a current which is far below the currents where the the $m=0$ modes are intersecting the $m=2$ modes (about $19 \mathrm{~A}$ for one mode, around $25 \mathrm{~A}$ for most others). Also, the growth rates due to coupling are well over an order of magnitude larger than their values when coupling is ignored (see figure 5.19; compare figure 5.18).

\subsection{6 $m=0$ Radial Mode Growth Rates}

Now, consider the $m=0$ radial modes. These are the modes which have a low-current frequency of $\omega_{y}$, but which have a form factor from equation (5.19) like the $m=2$ modes. They are called radial modes because if the longitudinal phase space density is observed for them, it has no azimuthal variation, but the density oscillates radially in the phase space. Figure 5.21 shows the growth rates of these modes as a function of current. Since they use 


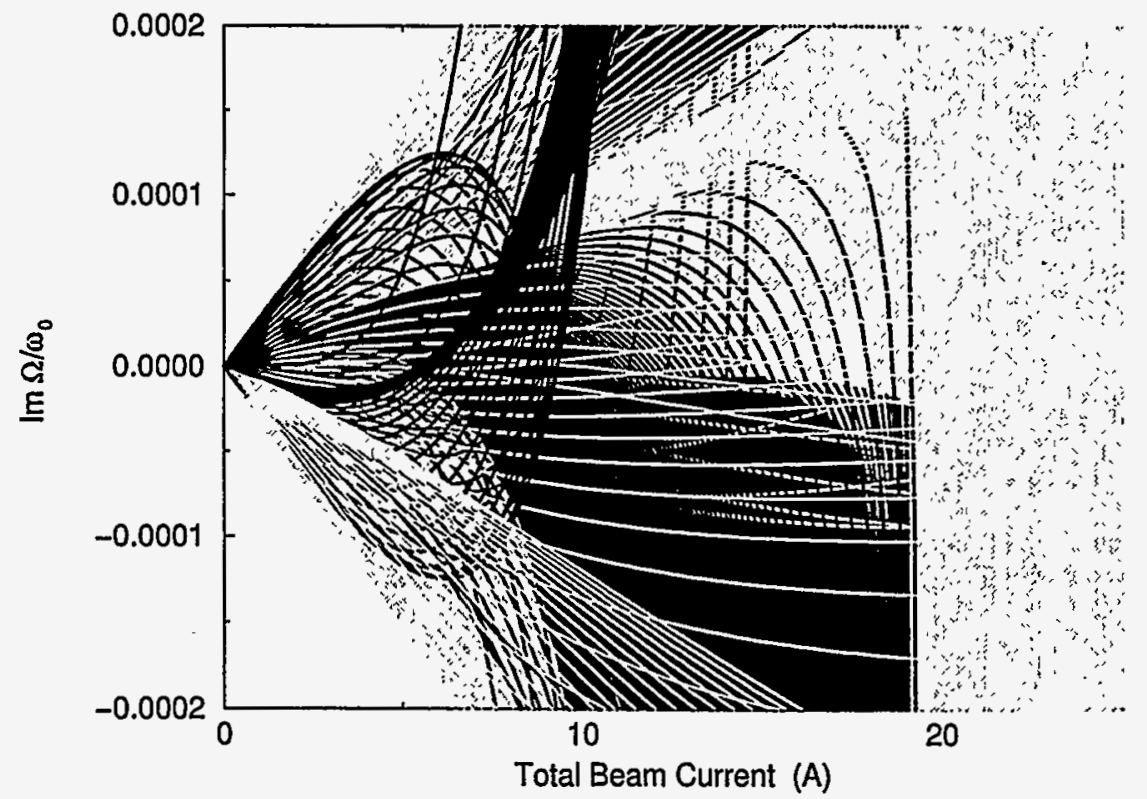

Figure 5.17: $m=1$ multibunch mode growth rates, with coupling. Vertical scale is blown up from figure 5.16

the same form factor as the $m=2$ modes, their growth rates only differ from those for the $m=2$ modes by a constant factor (about 2 ; see equation (4.83) ).

Figure 5.22 shows the growth rates of the $m=0$ radial modes when coupling is included. Note that the growth rates have increased nontrivially. However, the effect of mode coupling on these growth rates is qualitatively different from the effect seen previously (compare figures 5.15-5.17, 5.19, and 5.20): the growth rates still seem to increase linearly with current.

The reason for this can be seen by examining the 2 dimensional model for the eigenvalue system (5.9). Consider the coupling between the $m=0$ mode and the $m=0$ (first) radial mode. In that case, $k$ in equation (5.9) is zero. Thus, the mode frequency $\Omega$ is given by

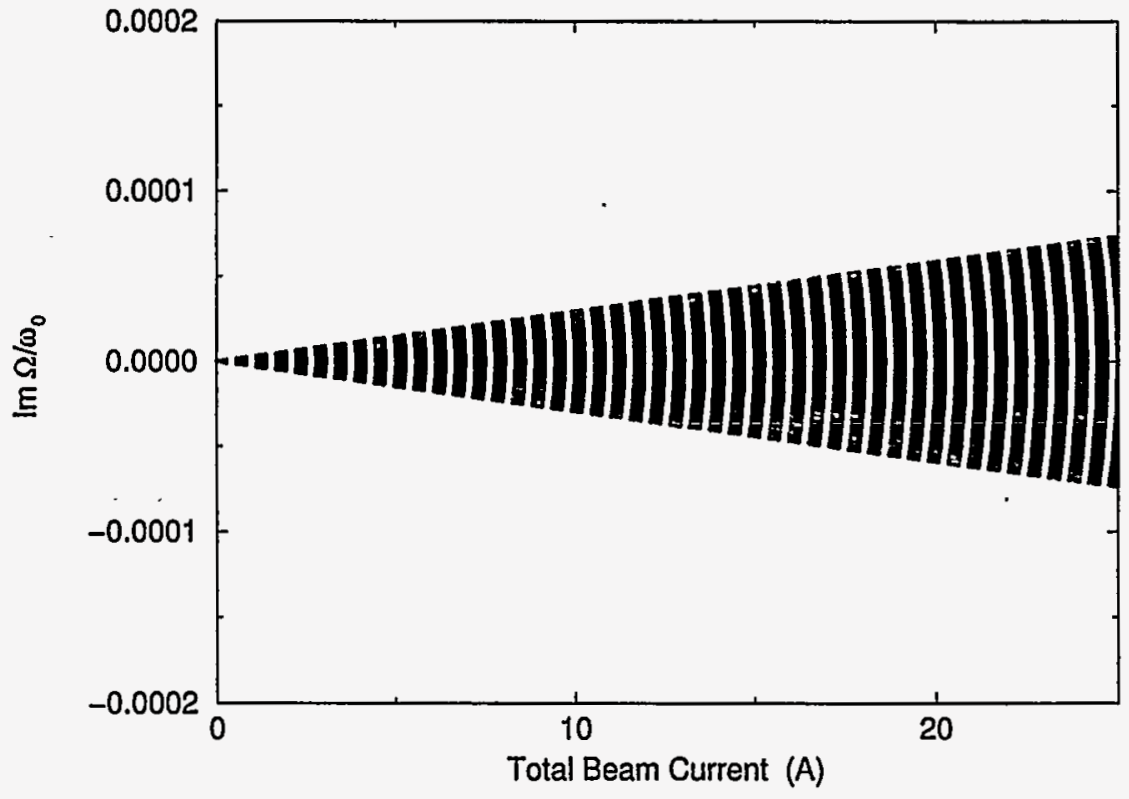

Figure 5.18: $m=2$ multibunch mode growth rates, without coupling.

(see equation (5.11) )

$$
\Omega=\frac{I}{2}\left(T \pm \sqrt{D^{2}+P}\right) .
$$

Notice that this is proportional to $I$. If $Z_{22}$ is the matrix element associated with the $m=0$ radial mode growth rate, and $Z_{11}$ is taken to be much larger than the other matrix elements (an excellent assumption as can be seen from the figures in this section), then the eigenfrequency of the $m=0$ radial mode with coupling is approximately

$$
I\left(Z_{22}-\frac{Z_{21} Z_{12}}{Z_{11}}\right)
$$

\subsection{7 $m=3$ Growth Rates}

The growth rates of the $m=3$ multibunch modes without coupling are shown in figure 5.23, and with coupling in figure 5.24. Qualitatively, the behavior is very similar to that of 


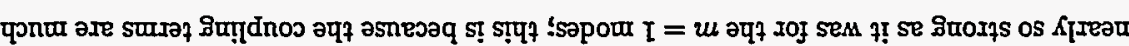

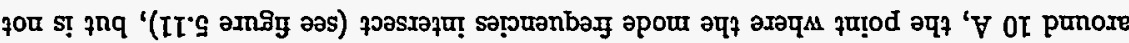

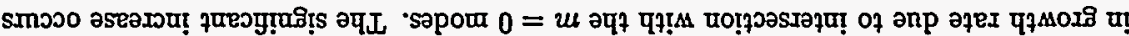

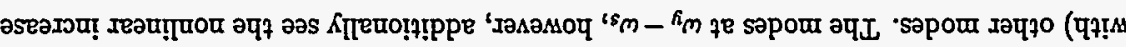

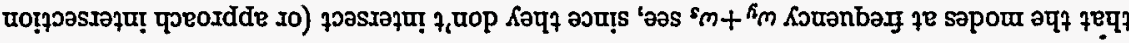

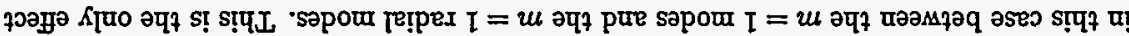

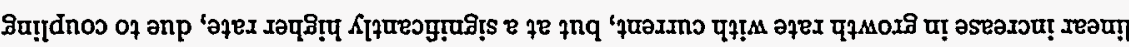

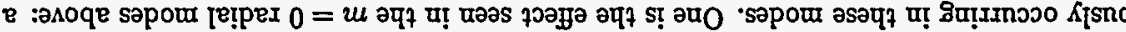

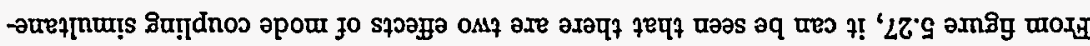

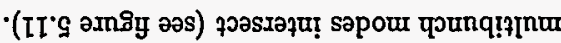

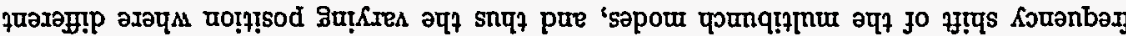

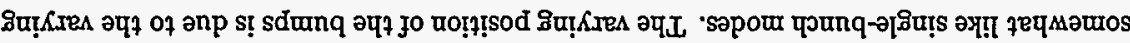

$6 \mathrm{I}^{\prime} \mathrm{g}$ amsig moxy

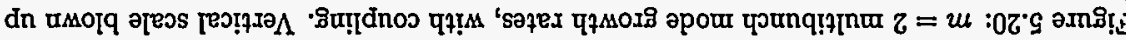

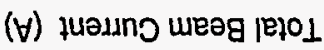

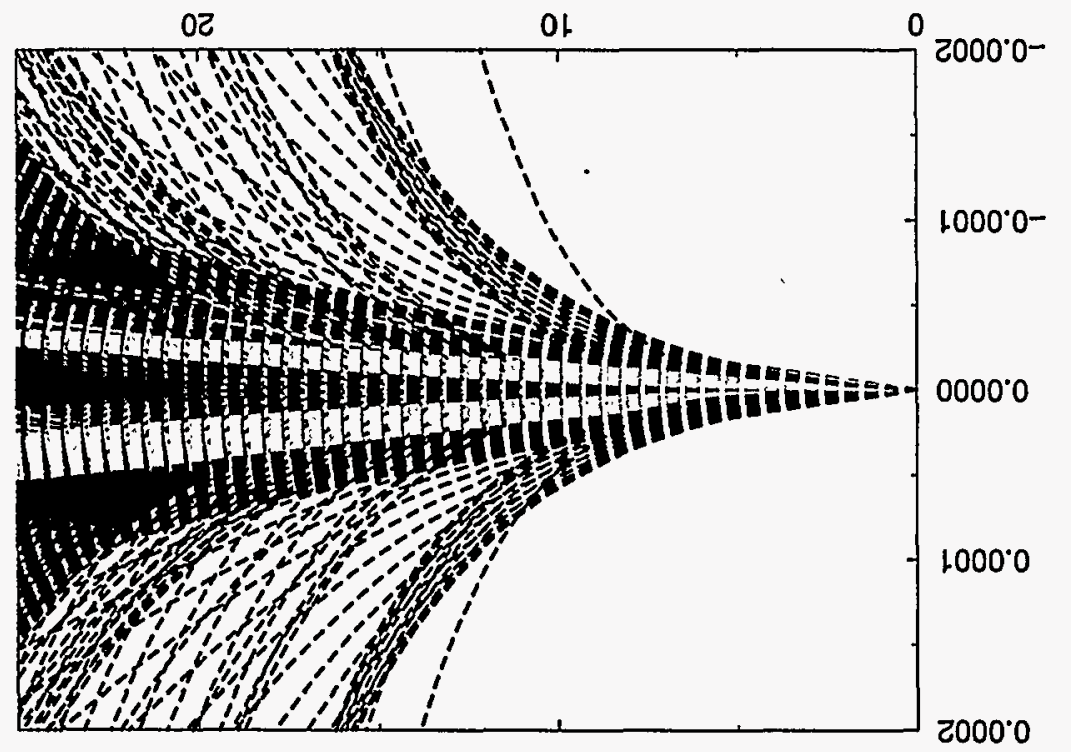

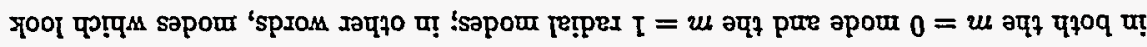

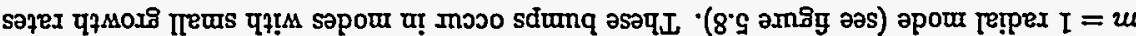

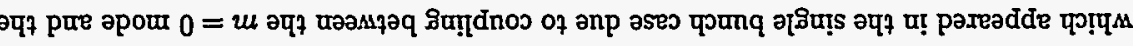

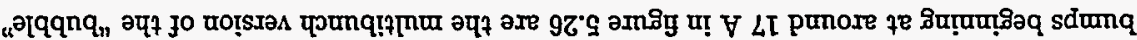

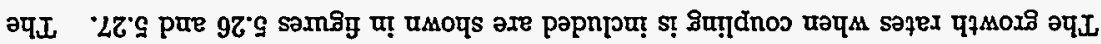

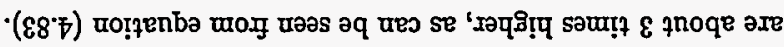

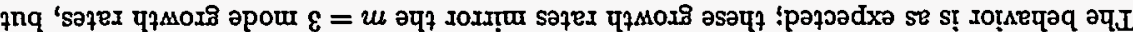

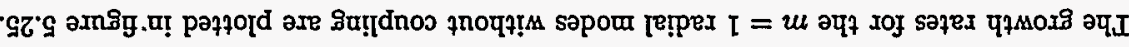

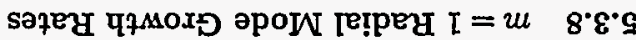

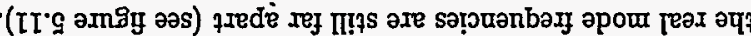

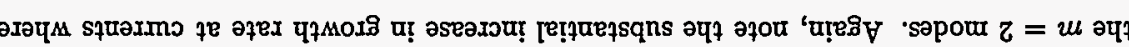

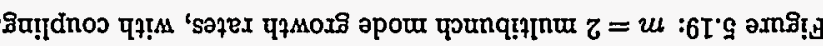

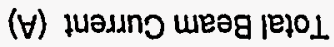

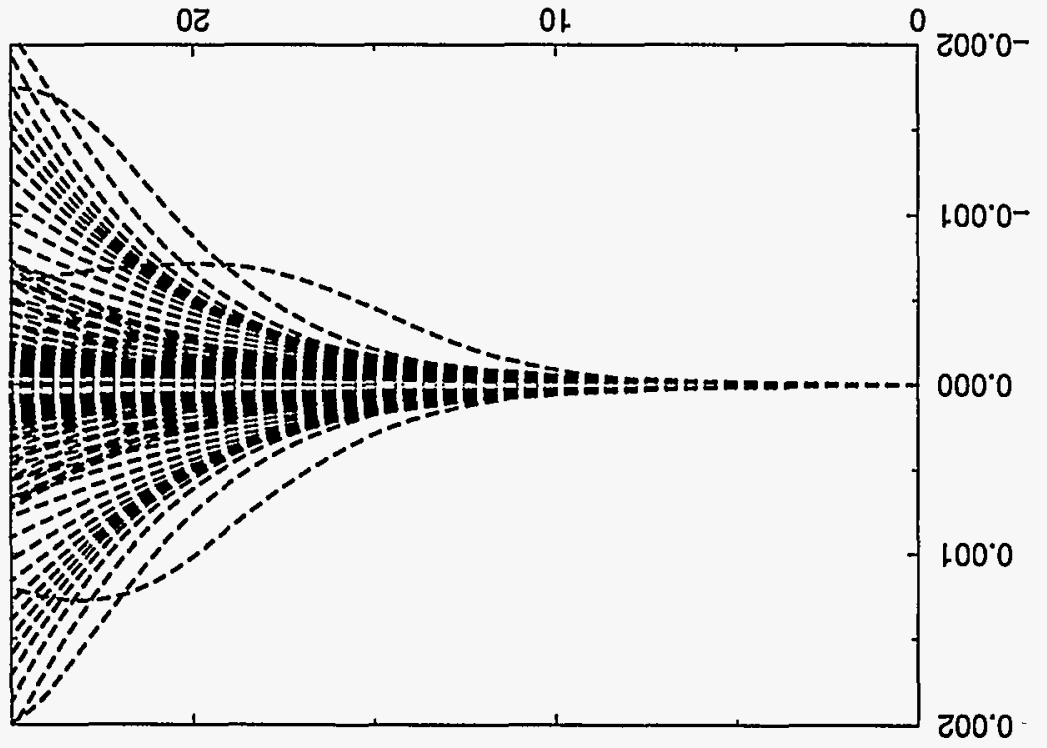




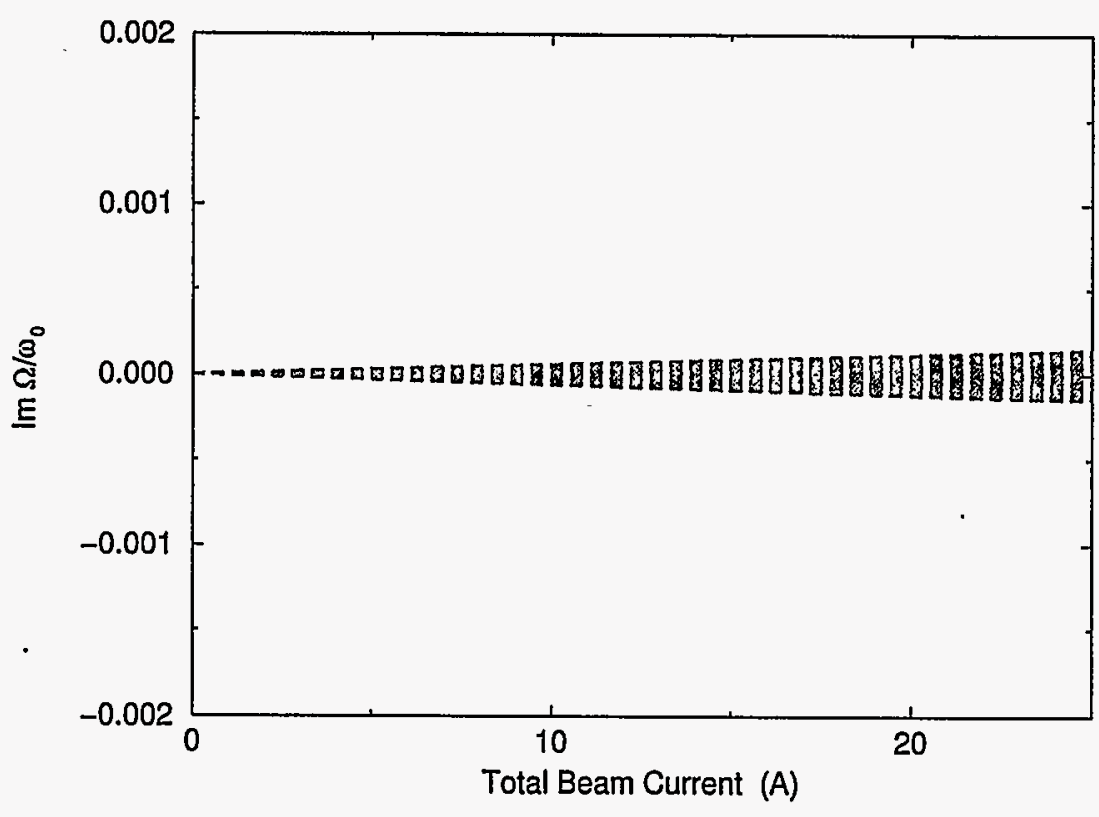

Figure 5.21: $m=0$ radial multibunch mode growth rates, without coupling.

smaller between the $m=0$ modes and the $m=1$ radial modes than they were with the $m=1$ (non-radial) modes (see equations (4.83) and (4.84)).

\subsubsection{Growth Rates for Fixed Current}

The worst-case operating current for the PEP-II B-factory will be $3 \mathrm{~A}$ [49]. As discussed in section 5.1, worst-case growth rates should be obtained by taking the current in the highestcurrent bunch, and assuming that the ring is symmetrically filled with bunches with that current. Assuming that the 1658 bunches in the ring all have the same current, this means that the total current of $3 \mathrm{~A}$ should be multiplied by $1746 / 1658$, giving an effective total current of $3.159 \mathrm{~A}$.

In figure 5.28 , the absolute values of the growth rates for the multibunch modes without coupling are plotted. The peaks in the figure reflect the peaks in the impedance (mainly due

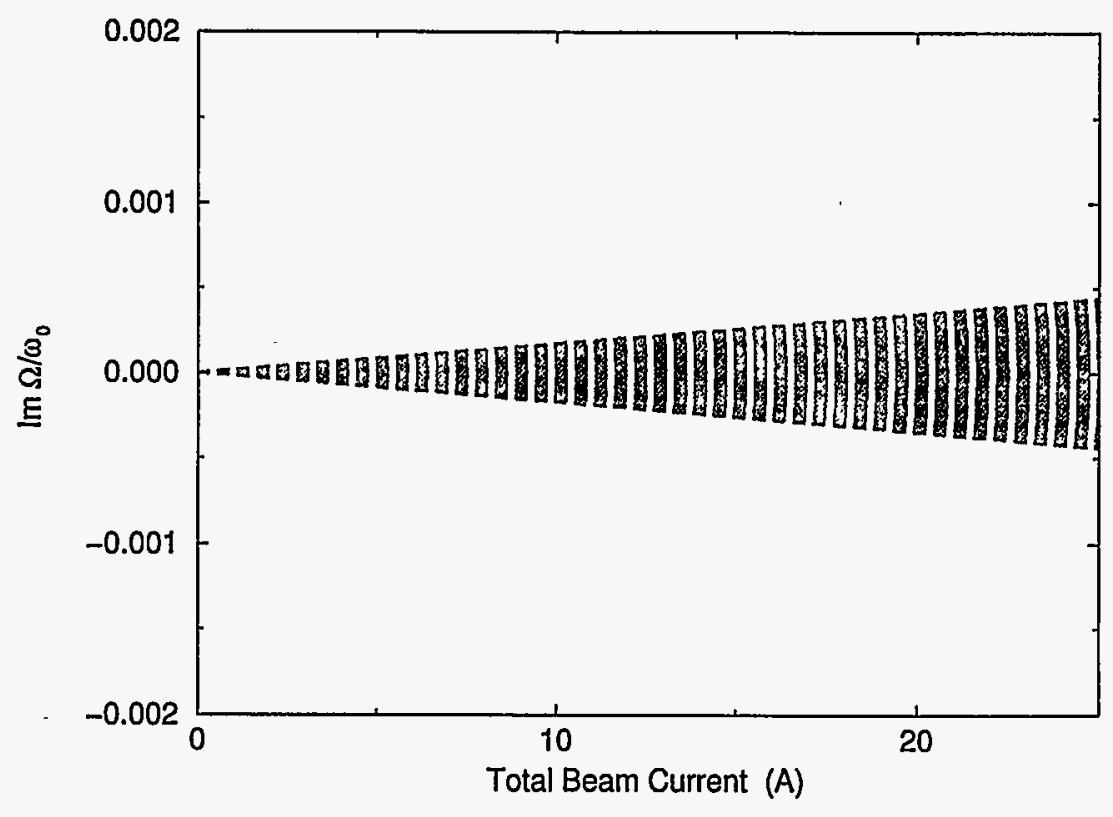

Figure 5.22: $m=0$ radial multibunch mode growth rates, with coupling.

to cavity modes and the resistive wall peak at $\omega \approx 0$ ). The summation over $q_{1}$ in equation (4.84) causes the modes to be interleaved in figure 5.28 from where they were in figure 5.2. Note that several of the $m=1$ modes are well above radiation damping, even when mode coupling is ignored. These unstable modes are caused by cavity higher order modes and the BPM resonance.

The results of introducing mode coupling are shown in figure 5.29. The $m=0$ growth rates, as expected, are almost unchanged. The pairs of modes which were degenerate in the uncoupled case (see figure 5.28) now have different growth rates. For some modes, the growth rates have increased significantly. The behavior of some of the modes has become dominated by coupling: in particular, the $m=0$ and $m=1$ radial modes; this is evident from the fact that the modes vary with mode number rather differently than they did in the uncoupled case. Note that some of the $m=1$ modes have, due to coupling, risen above 

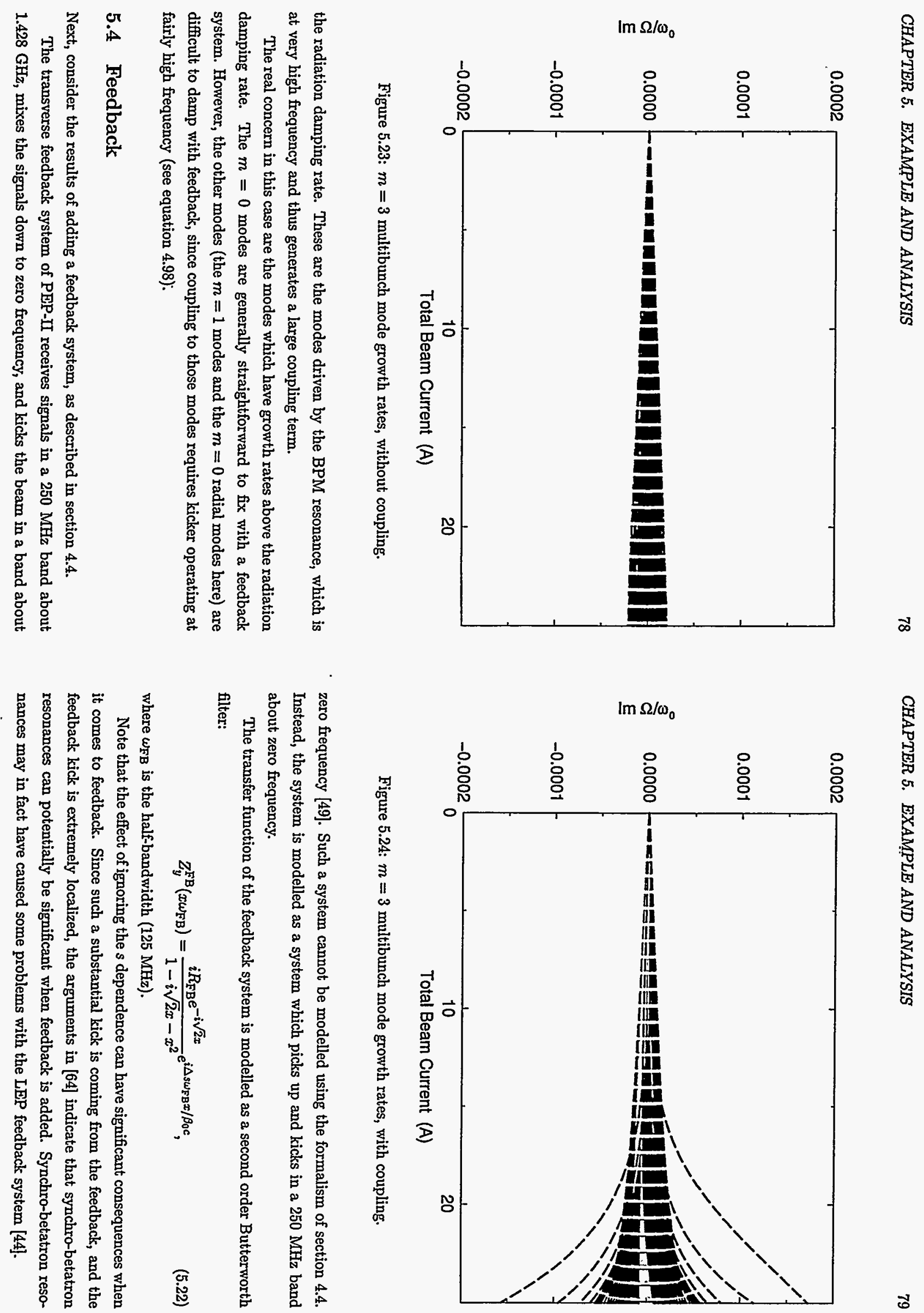


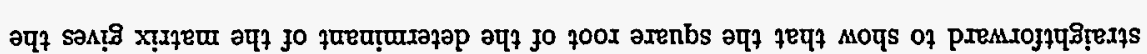

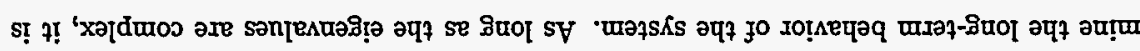

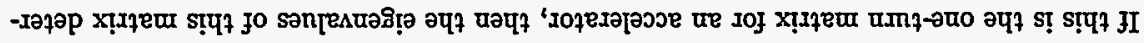
$\left(7 Z^{\circ} 9\right)$

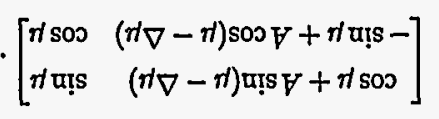

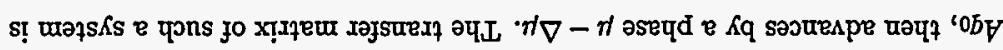

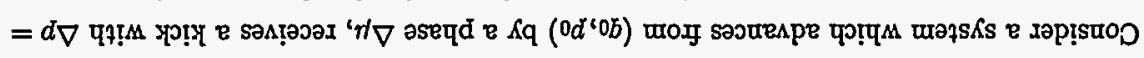
(\&६s) $\quad\left[\begin{array}{l}0 d \\ 0 b\end{array}\right]\left[\begin{array}{cc}\pi \nabla \text { sos } & \pi \nabla \text { u!s }- \\ \pi \nabla \text { u!s } & \pi \nabla \text { sos }\end{array}\right]=\left[\begin{array}{l}d \\ b\end{array}\right]$

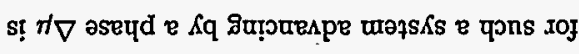

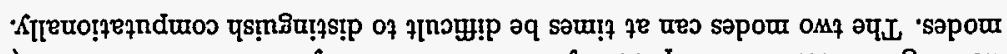

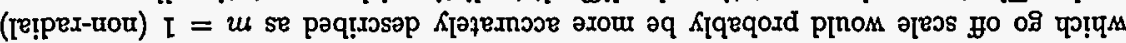

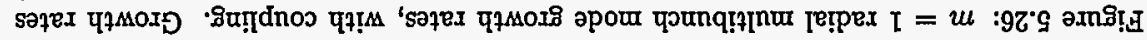

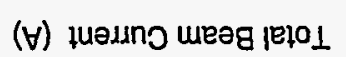

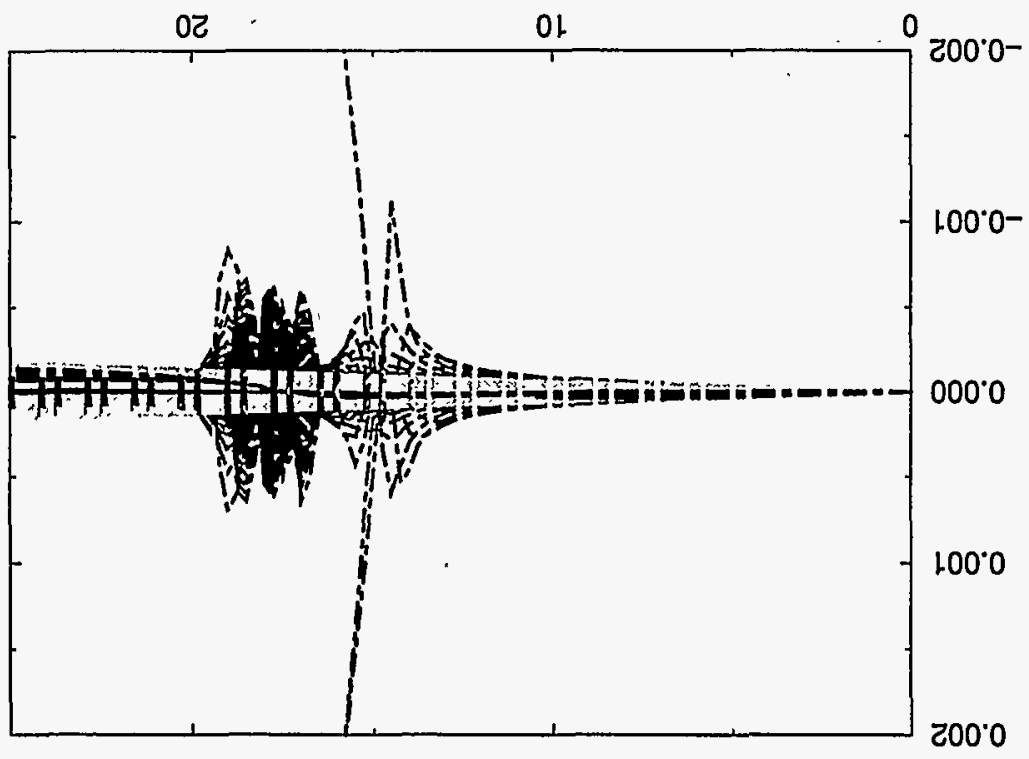

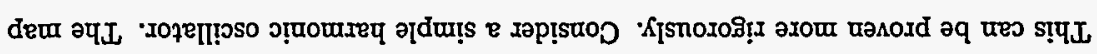

'unquaurom oqu! pauriojsurext

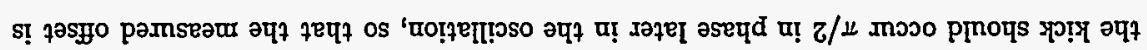

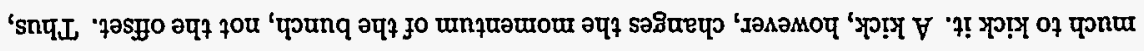

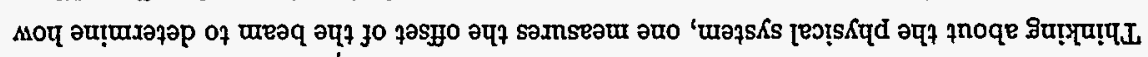

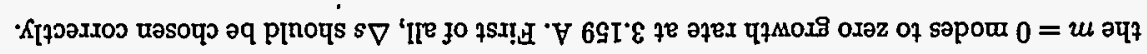

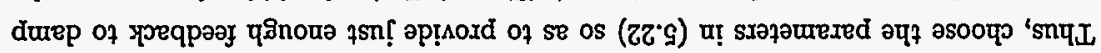

$\cdot\left(\left(66^{\circ} \overline{)}\right)\right.$

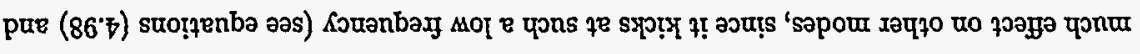

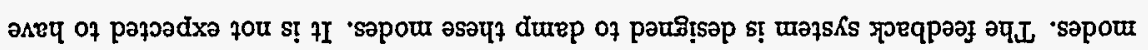

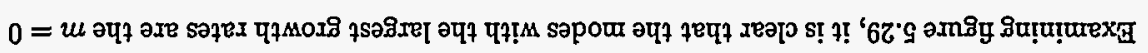

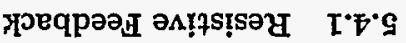

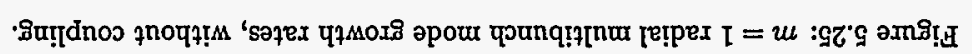

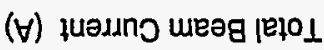

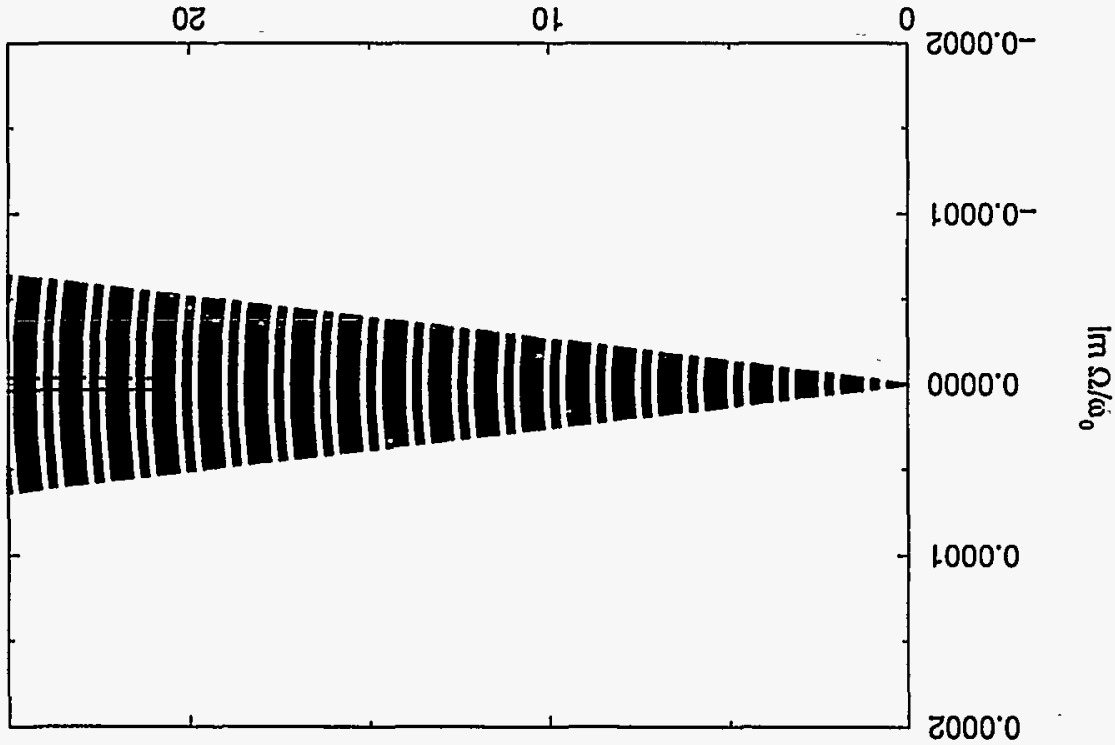

08 


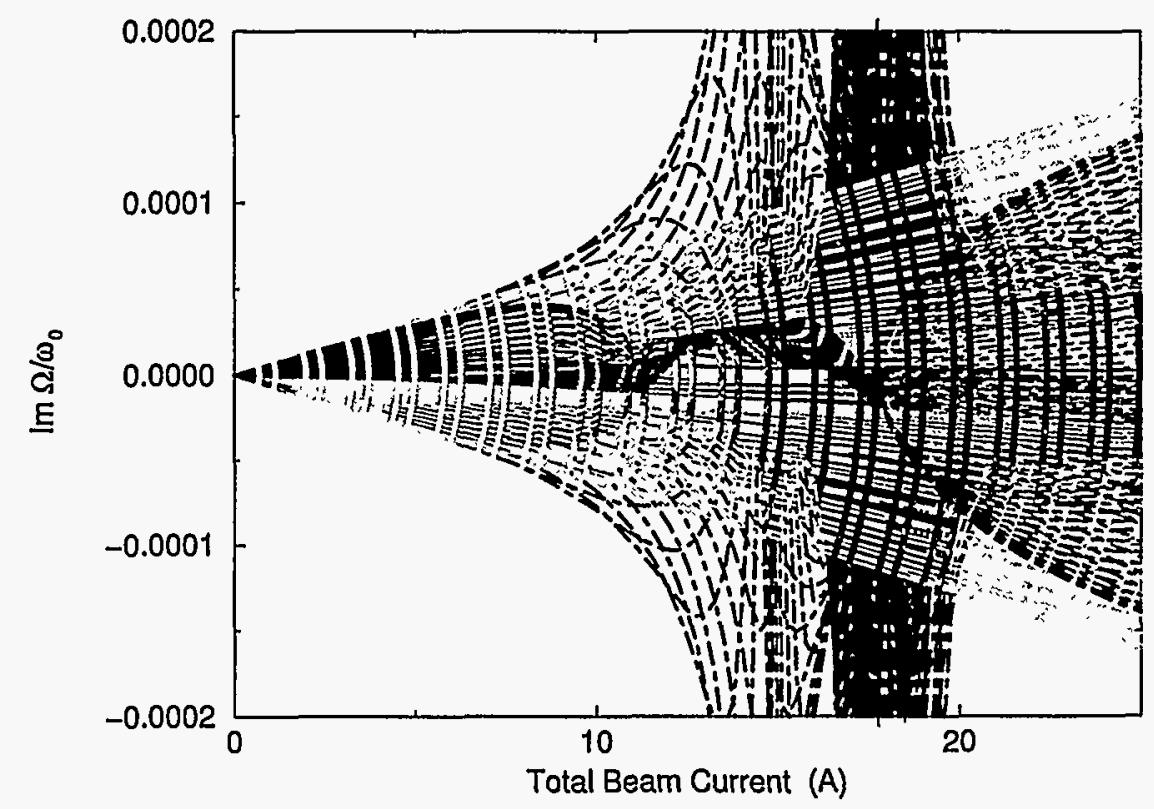

Figure 5.27: $m=1$ radial multibunch mode growth rates, with coupling. Vertical scale blown up from figure 5.26 .

absolute value of the eigenvalues. The determinant of (5.24) is $1-A \sin \Delta \mu$. Thus, the damping rate is clearly maximized when $\Delta \mu=\pi / 2$. It can be seen that this result holds for the general linear one-dimensional as described in appendix A.3 by simply replacing $q$ and $p$ with $q / \sqrt{\beta}$ and $\sqrt{\beta} p+\alpha q / \sqrt{\beta}$.

Another way to see that such a phase shift will produce damping is by examining the equations for the eigenvalues. First, note that the $Z_{y}^{\mathrm{FB}}$ given by equation (5.22) is mostly imaginary (see figure 5.30). Next, the combinations of the exponentials in (5.22) and (4.98) effectively give a factor of $e^{i\left[\psi_{y}(s)-\psi_{y}(s-\Delta s)\right]}$ (ignoring corrections of order $\psi_{\tau}(s)-\psi_{\tau}(s-\Delta s)$ ). If the phase advance from pickup to kicker is $\pi / 2$, this gives another factor of $i$. From equation (4.83), there is another factor of $-i$. The net result is (approximately) that the feedback will add a negative imaginary part to $\Omega$, giving damping, as long as $\Delta s$ is chosen so as to give a $\pi / 2$ phase advance from pickup to kicker.

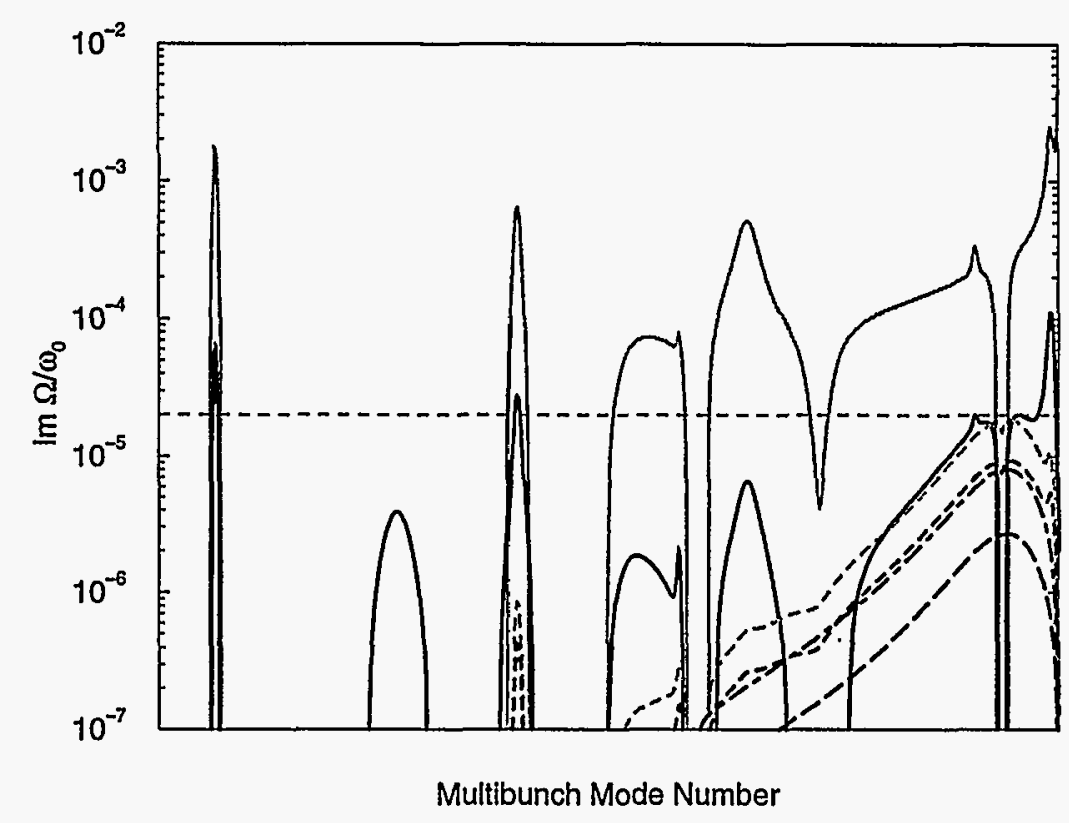

Figure 5.28: Multibunch mode growth rates, without coupling, at 3.159 A. There are 1746 modes shown, with a line connecting them (modes with negative growth rates are off-scale) In some cases, pairs of modes (e.g., the two $m=1$ modes) overlap. The horizontal dashed line gives the radiation damping rate.

Next, one must choose the amplitude $R_{\mathrm{FB}}$. For this example, it was chosen by making a run for $3.159 \mathrm{~A}$ with feedback and no impedance, and taking the ratio of the minimum damping rate of all the modes to the maximum damping rate of all the modes for the case with all the impedance and no feedback.

Figure 5.31 shows the multibunch mode frequencies when feedback is included. There are very few changes in this diagram from figure 5.11 . The frequencies have shifted slightly (partly because (5.22) is not purely imaginary, partly due to mode coupling), and one mode is displaying behavior similar to a "bubble" from single-bunch mode coupling. This will be discussed momentarily.

Figure 5.32 demonstrates that the feedback system has accomplished its task of damping 


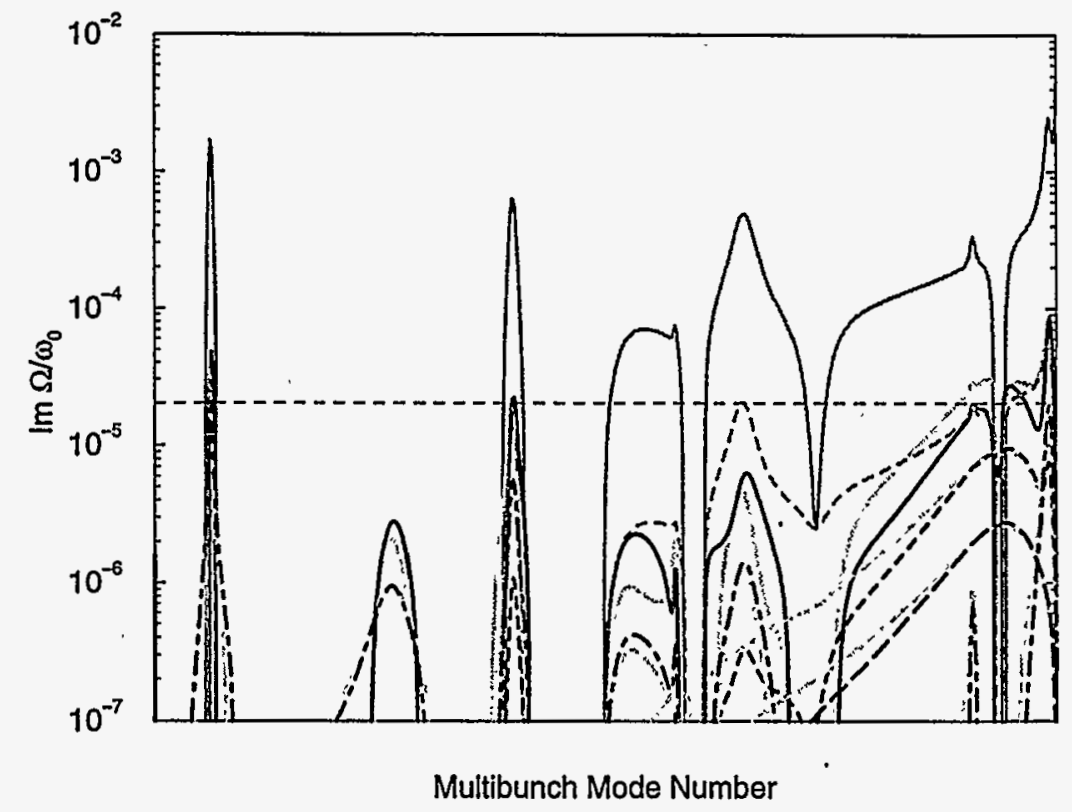

Figure 5.29: Multibunch mode growth rates, with coupling, at $3.159 \mathrm{~A}$.

the $m=0$ modes. One of the modes, however, has just barely been damped enough (in fact, it still has a small positive growth rate). Since the imaginary part of its mode frequency is nearly zero, the behavior of its growth rate is very similar to what is seen in single-bunch mode coupling (compare figures 5.6, 5.8, and 5.9). This gives a "bubble" in the growth rate at the point where the mode frequencies coincide. This could be avoided by increasing the feedback gain, giving the mode a large negative imaginary part. At the operating current of $3 \mathrm{~A}$, however, it is unnecessary.

The feedback system has no beneficial effect on the $m=1$ modes, however, as can be seen from examining figures 5.33 and 5.34. This is the expected behavior, since the feedback impedance has no substantial high-frequency components. Actually, the feedback system seems to have actually increased some of the growth rates, as can be seen from more careful examination of figures 5.33 and 5.34 . This is a result of multibunch mode coupling

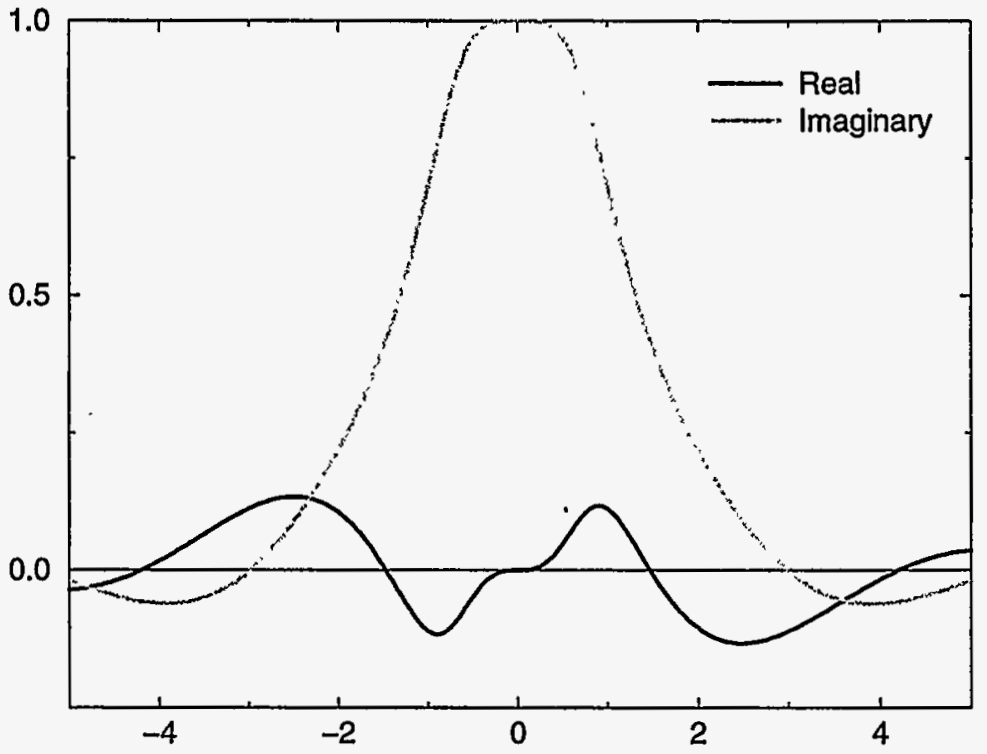

Figure 5.30: Scaled feedback impedance from equation (5.22).

combined with the feedback; figure 5.35 demonstrates that the growth rates are symmetric about zero when coupling is ignored. Finally, it appears that some of the modes also have smaller growth rates in the presence of feedback, despite the fact that feedback should not directly affect the $m=1$ modes. This is because the frequencies of many of the $m=0$ modes have developed such a large imaginary part that the coupling to the $m=1$ modes has weakened for them. Overall, however, the $m=1$ modes are still increasing sharply at about the same point, and they are increasing by pretty much the same amount, at least for currents close to the "threshold" (around $10 \mathrm{~A}$ ).

The $m=0$ radial modes are also well damped by the feedback system, as shown in figure 


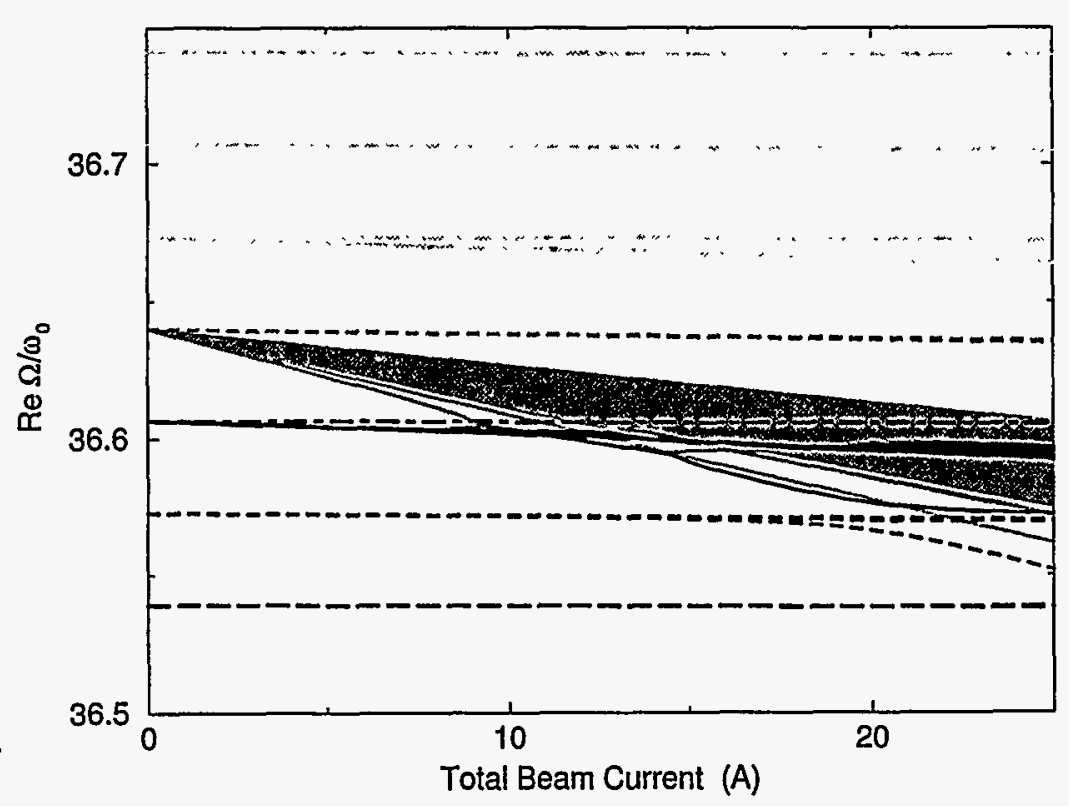

Figure 5.31: Multibunch mode frequencies, with feedback.

5.36. This is a direct effect of multibunch mode coupling combined with the feedback's effect on the $m=0$ modes. The effect can be fairly well understood from equation (5.21); when the imaginary part of $Z_{11}$ becomes negative, $Z_{11}$ in the denominator combined with the minus sign gives an additional damping to the radial modes.

The growth rates for the various modes are shown in figure 5.37. Although not necessarily obvious from this figure, the $m=0$ and $m=0$ radial modes are damped, while some of the $m=1$ modes are still well above radiation damping. The feedback system was of little help to these $m=1$ modes.

\subsubsection{Reactive Feedback}

To avoid the effects of transverse mode coupling, one can create a feedback system which compensates the shift in frequency of the $m=0$ mode so as to increase the threshold where

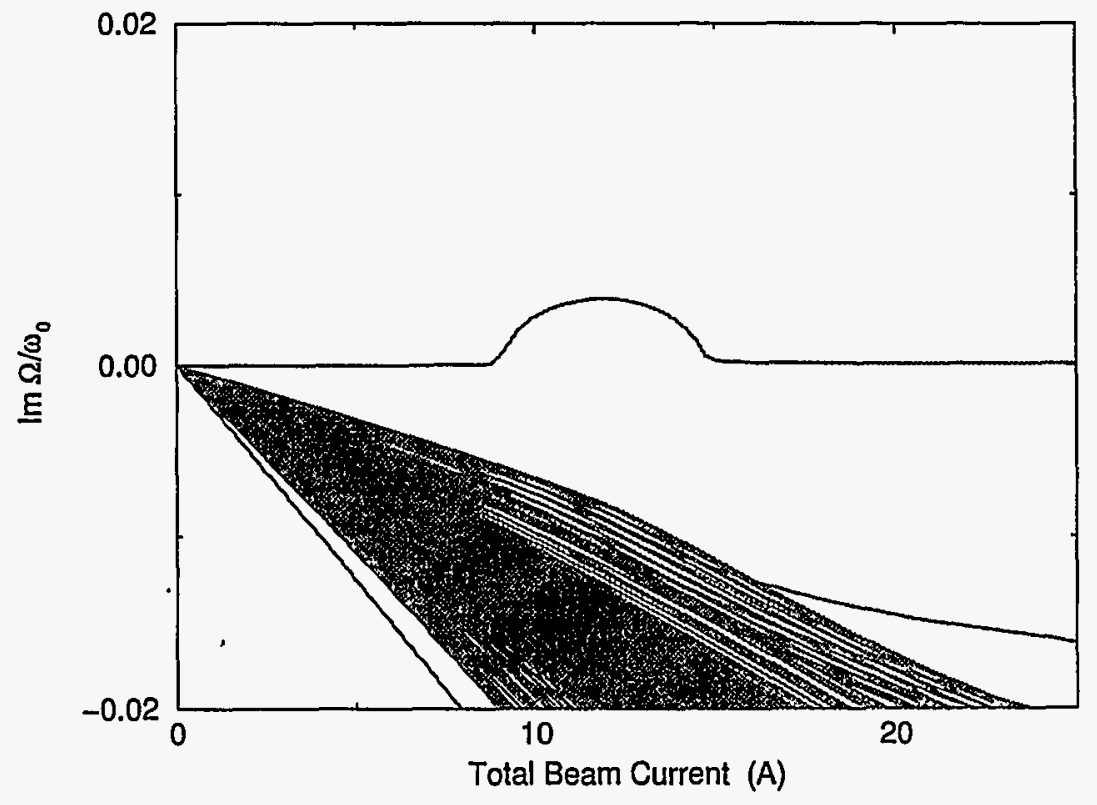

Figure 5.32: $m=0$ multibunch growth rates, with feedback.

the $m=0$ mode frequency coincides with the frequency of the $m=1$ (radial) modes. This is known as a reactive feedback system, and has been suggested before as a cure for the transverse mode coupling instability $[44,52,56]$.

The improvement to be expected from reactive feedback is significant, but not nearly as large as would be expected from a single-bunch analysis. The reason can be seen by comparing the single-bunch mode frequencies of figure 5.6 to the multibunch mode frequencies in figure 5.11. If there is only a single bunch, there is only one frequency associated with the $m=0$ mode, and so that frequency can be shifted to guide the $m=0$ mode between the $m=1$ modes, at least up to a point. However, in the multibunch case, there is a "fan" of modes, which has a finite divergence. A reactive feedback will more or less shift every mode by the same amount. Thus, there is a limit to how high the current can get before the $m=0$ lines will be forced to cross one of the $m=1$ lines, no matter what amount of 


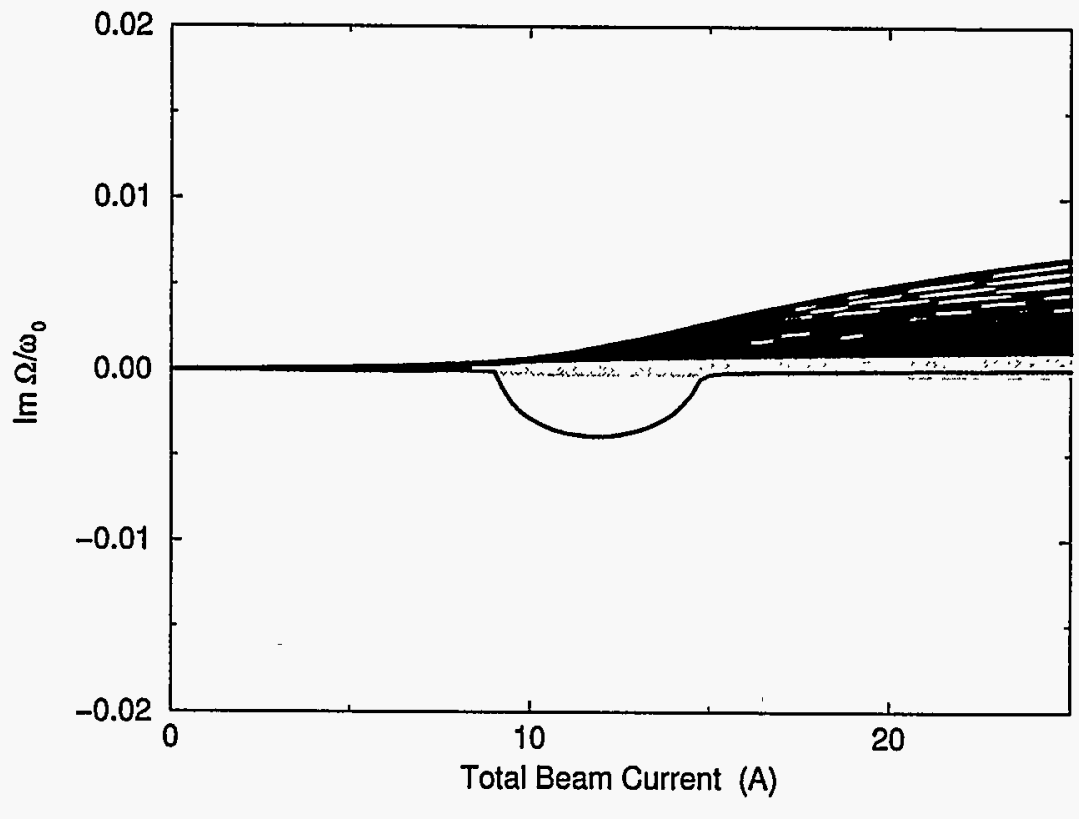

Figure 5.33: $m=1$ multibunch growth rates, with feedback.

reactive feedback is used.

The discussion above indicates that if the (betatron) phase shift from pickup to kicker is chosen to be a multiple of $\pi$, then there will be no damping or anti-damping from the feedback system. There will be a frequency shift, however. The one-turn tune $\mu^{\prime}$ can be determined from the eigenvalues of (5.24) to be given by $\cos \mu^{\prime}=(1 \pm A / 2) \cos \mu$ when $\Delta \mu$ is a multiple of $\pi$.

The analysis of the effect of reactive feedback is performed by adding reactive feedback to the system above with resistive feedback. The amplitude of the feedback is determined by finding the frequency shift of the $m=0$ mode at $3 \mathrm{~A}$ for the single bunch case shown in figure 5.6, finding the frequency shift due to feedback alone, and multiplying the amplitude by the ratio of the frequency shifts.

The mode frequencies that result are shown in figure 5.38. Note that the $m=0$ mode

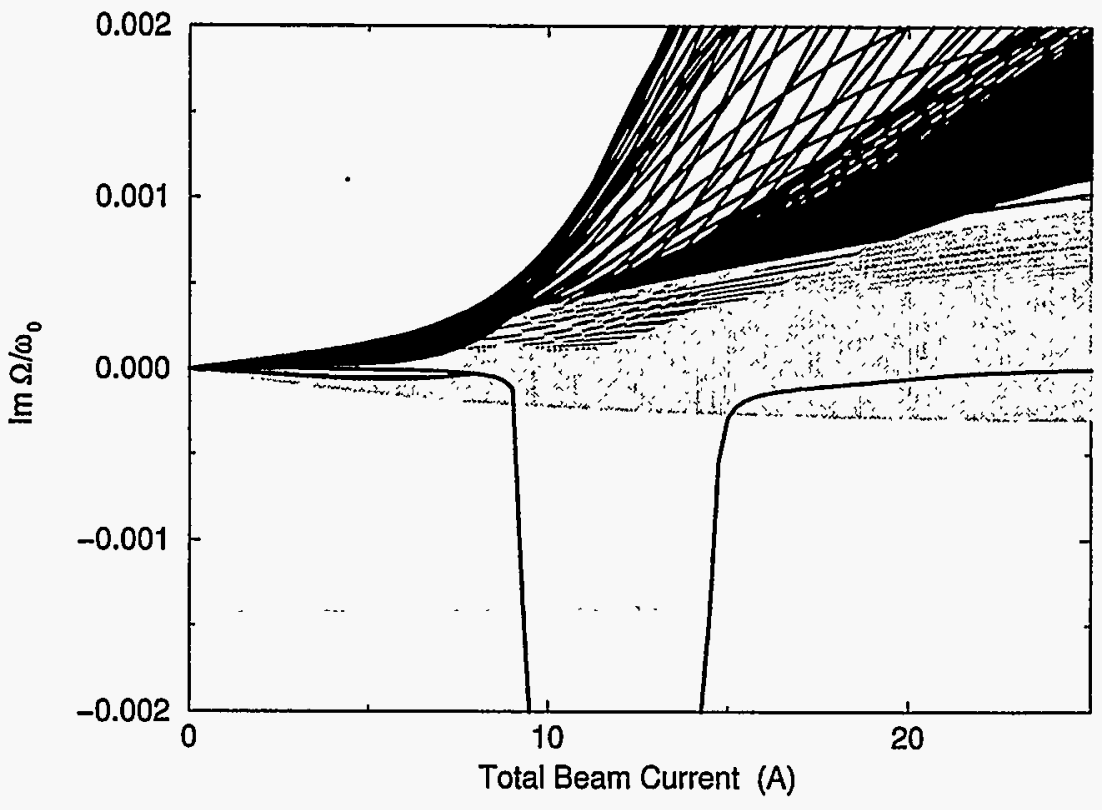

Figure 5.34: $m=1$ multibunch growth rates, with feedback. Vertical scale blown up from figure 5.33.

frequencies have been shifted up, and that now the lowest current at which the modes coincide is around $17 \mathrm{~A}$. The frequencies could clearly have been shifted even more, raising that threshold further. However, there is clearly a limit of around $25 \mathrm{~A}$ where the modes would be forced to intersect, either with the $m=1$ modes at $\omega_{y}+\omega_{s}$, or the $m=1$ modes at $\omega_{y}-\omega_{s}$.

The $m=0$ radial modes have acquired an increased frequency spread due to this, since whereas the $m=0$ (non-radial) modes originally drifted away from the $m=0$ radial modes, they now follow them fairly closely, increasing the coupling effect. The spread in the imaginary part of the frequencies also has increased significantly, as can be seen in figure 5.39. Luckily, the feedback damping of the $m=0$ modes still damps the $m=0$ radial modes through coupling.

Reactive feedback has succeeded in its desired effect of reducing the effect of multibunch 


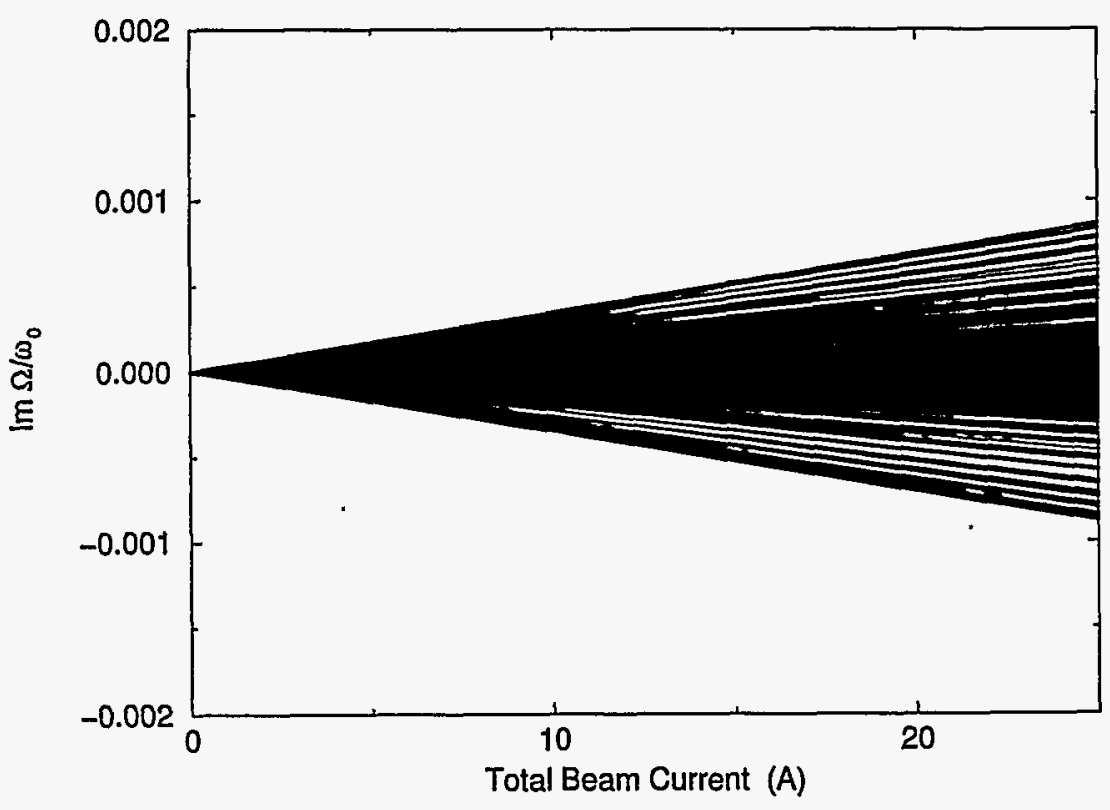

Figure 5.35: $m=1$ multibunch growth rates, with feedback but no coupling.

mode coupling on the $m=1$ modes, as can be seen from figure 5.40. The growth rates are increased slightly from the uncoupled case (see figure 5.14), but the effect is insignificant compared to the increase without the reactive feedback (see figure 5.16). As can be seen from figure 5.38, the bulk of the $m=0$ modes haven't even intersected the $m=1$ modes yet at $25 \mathrm{~A}$, and so the increases in growth rates are not yet very sharp at the currents shown in figure 5.40. A similar improvement is seen in the $m=1$ radial modes, as shown in figure 5.41 (compare figure 5.27).

Finally, the nonlinear increase in growth rates at low currents due to mode coupling doesn't seem to have improved much. This can be seen by examining figure 5.42 . Note that there has been little improvement in the $m=1$ growth rates that were enhanced by mode coupling (compare figures 5.28 and 5.29 ).

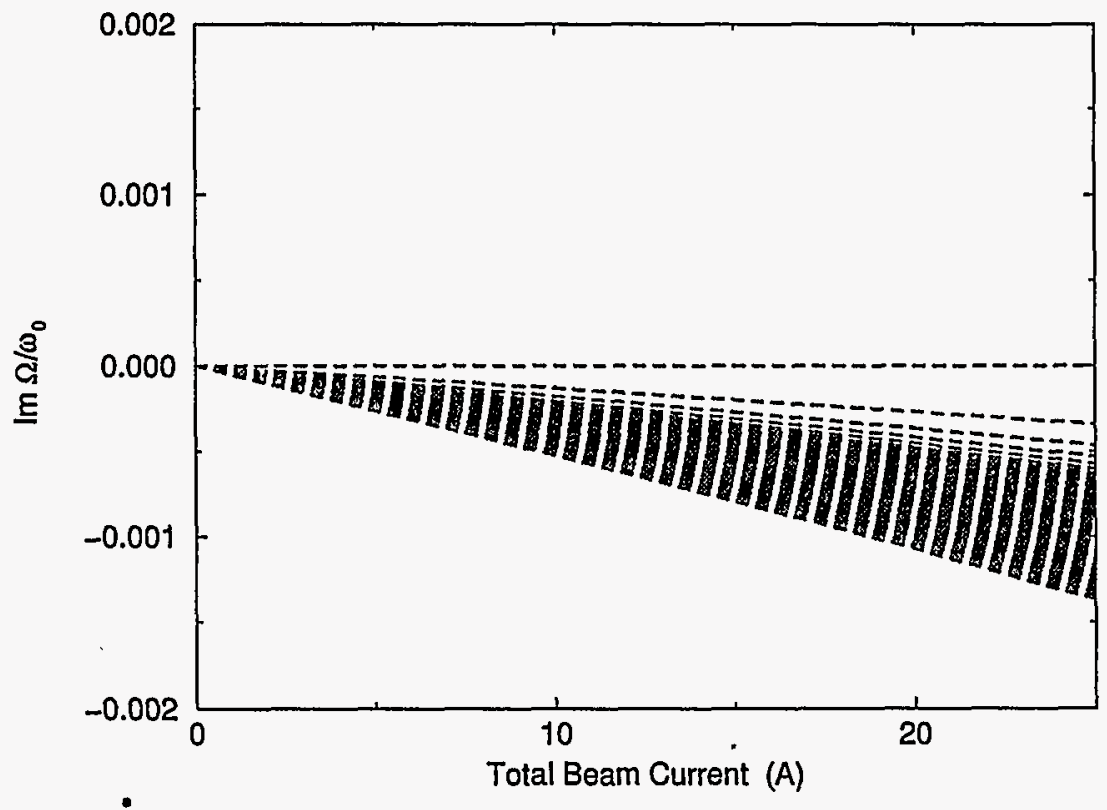

Figure 5.36: $m=0$ radial multibunch growth rates, with feedback.

\subsection{Landau Damping}

As pointed out above, the synchrotron tune shift at the longitudinal emittance is $-4.4 \times 10^{-5}$, which is somewhat above radiation damping. Thus, one would hope for some Landau damping of the modes. However, this does not turn out to be the case.

Consider the analysis of Landau damping due to synchrotron tune shift in [70]. If the absolute value of the tune shift is less than $6.8 \times 10^{-5}$, then the modes should be Landau damped. Figure 5.43 shows the absolute values of the multibunch modes with coupling. As can be seen, the absolute values are much larger than $6.8 \times 10^{-5}$. The reason is that there is a large tune shift due to the broadband impedance, whereas the growth rates are due only to narrow band impedances (the broadband contribution to the grow rate cancels approximately due to the anti-symmetry of the real part of the transverse impedance-see equation (4.84) ). 


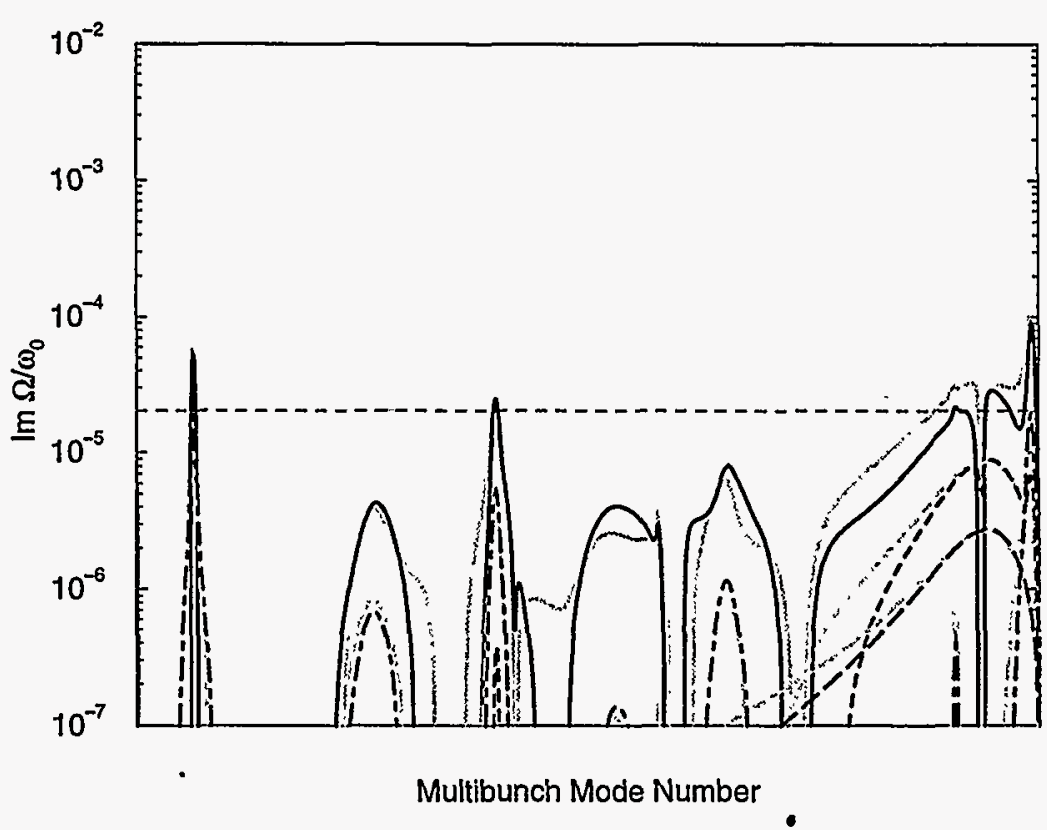

Figure 5.37: Multibunch mode growth rates at $3.159 \mathrm{~A}$, with feedback.

While one can rely more carefully on the stability diagram instead of just the absolute value (see [70]), it is fairly clear from the stability diagram that the stability limit from Landau damping at the large tune shift here is smaller than the radiation damping rate [70].

Thus, no improvement from Landau damping due to synchrotron tune spread is expected for the PEP-II low energy ring. The same reasoning should apply to the bunch-to-bunch tune variation.

\subsection{Chromaticity}

As described above, the effect of chromaticity can be estimated by shifting the frequencies of the form factors in (4.84). Every occurrence of $\omega$ in (4.84) is replaced by $\omega-\omega_{\xi}$, with the exception of the $\omega$ appearing in $Z_{\perp}$. This has been the approach of most every other author $[11,38,40,57-59,63,66,67,70]$. It may in fact be the correct thing to do in the

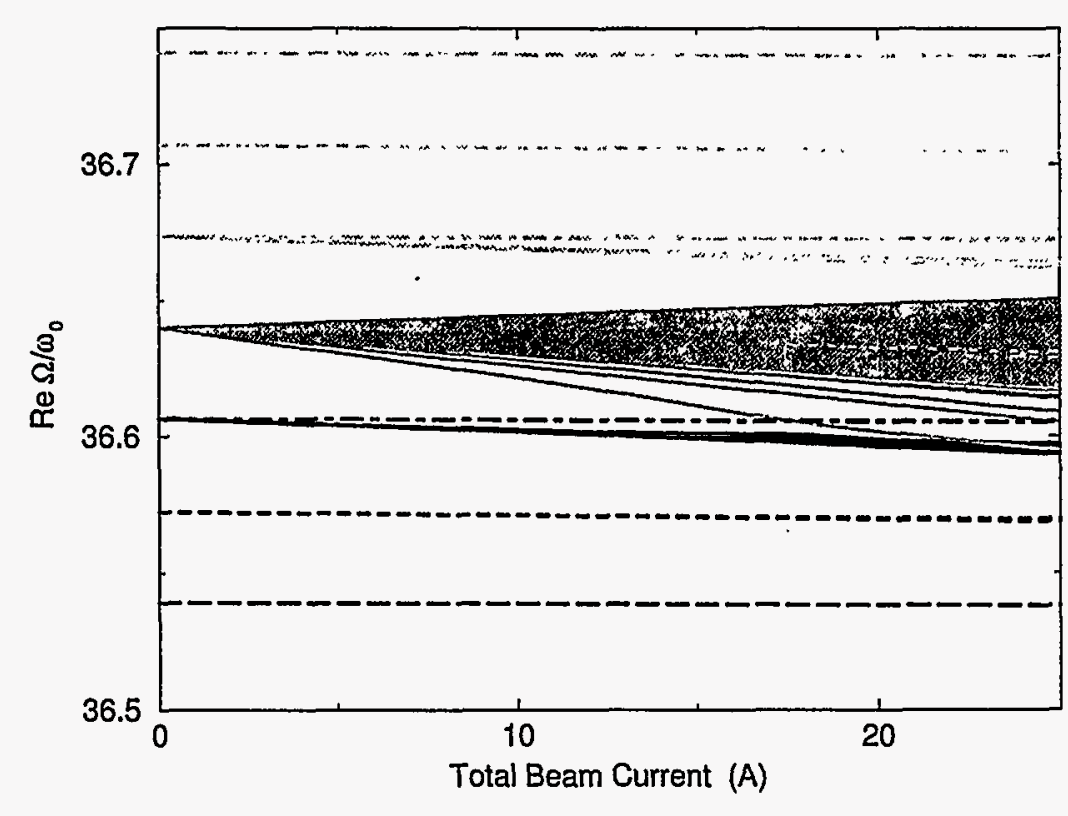

Figure 5.38: Multibunch mode frequencies with both resistive and reactive feedback.

formalism presented here; however, the computation has not been completed at this point.

Adding a small amount of chromaticity ( $\xi=2$ in the example here) has only a minor effect on single-bunch mode coupling in this system, as can be seen in figures 5.44 and 5.45 (compare figure 5.6 and 5.8). Figure 5.45 does show that the modes have nonzero growth rates at currents below the point where the modes intersect. This is even more prominent when the scales are blown up, as shown in figure 5.46. The "bubble" due to the coupling between the $m=0$ mode and the $m=1$ radial mode has been reduced significantly due to the introduction of chromaticity (see figure 5.45; compare figure 5.8). The reason is that the growth rates due to chromaticity are comparable to the growth rates due to mode coupling at that current. The spread in frequency that is associated with the finite growth rates effectively reduces the oscillation amplitude that one mode sees due to another, and thus reduces the resonant driving. 


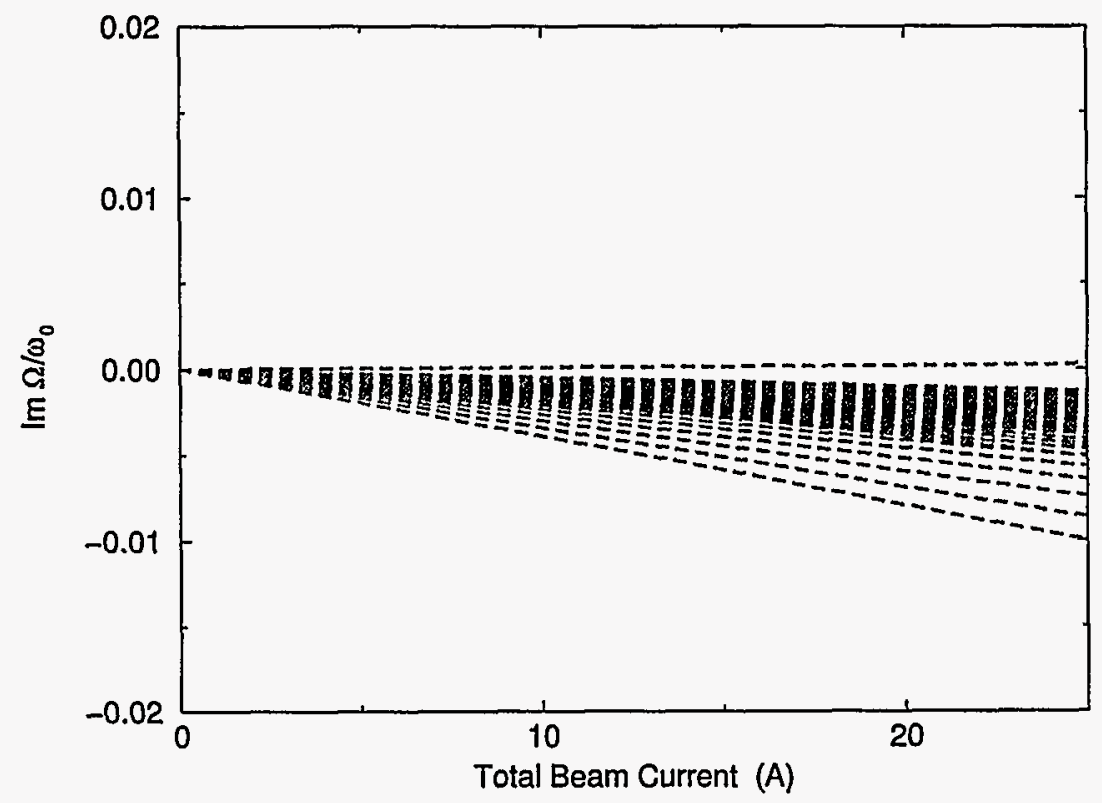

Figure 5.39: $m=0$ radial mode mode growth/damping rates with resistive and reactive feedback. Note that the vertical scale is larger than in figure 5.36 .

Note that for low currents, all the modes are damped. The chromaticity has broken the symmetry that gave near-zero growth rates. A positive chromaticity gives damping because the form factors (see figures 5.4 and 5.5) are shifted to the positive $\omega$ side, where the real part of the transverse impedance is positive (see figure 5.2, and recall that $Z_{\perp}(-\omega)=$ $-Z_{\perp}(\omega)$ for $\omega$ real). A positive real impedance leads to damping, as can be seen from equations (4.83) and (4.84). Thus, since a greater part of the form factor is in a region with positive impedance when the chromaticity is positive, there is a damping of the modes. This mechanism could in principle be defeated if, for instance, the positive $\omega$ peak of the $n+n^{\prime}=2$ form factor were shifted so that it no longer overlapped the bulk of the impedance on the positive side, whereas the negative $\omega$ peak overlapped the bulk of the impedance on the negative side. This damping mechanism is also defeated if the impedance is decreasing in the region where the form factor is nonzero; then the shift to the right causes the form

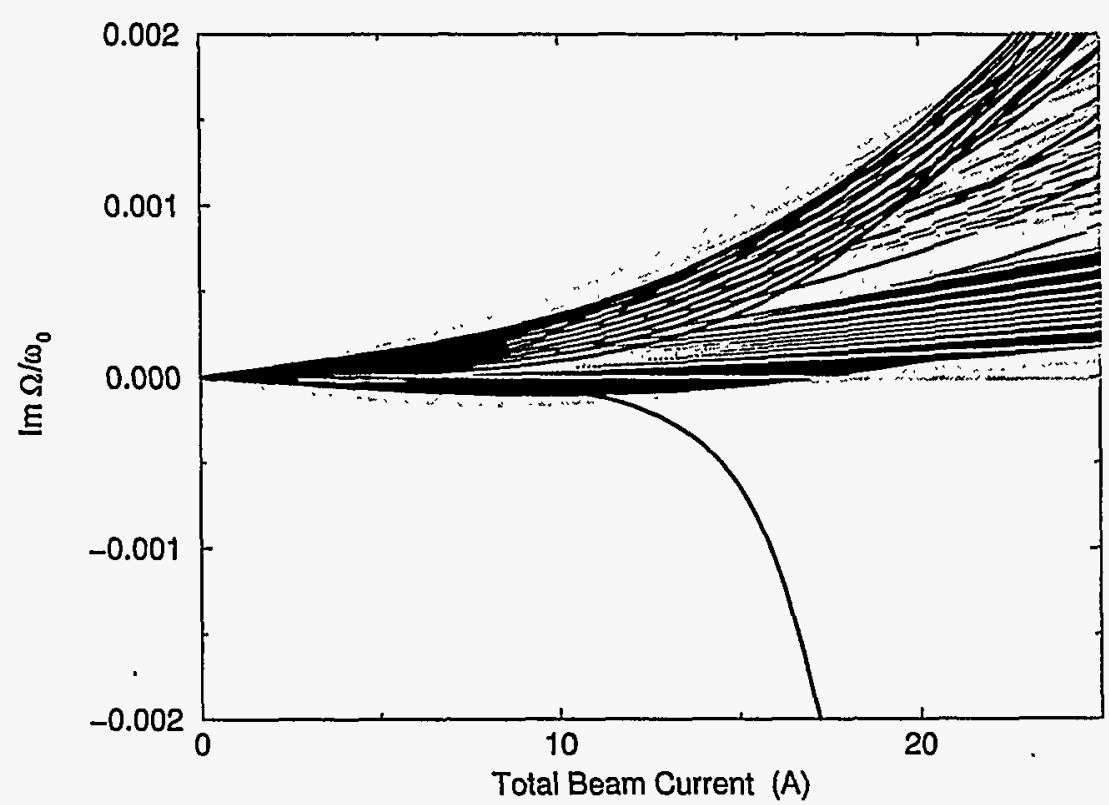

Figure 5.40: $m=1$ multibunch mode growth/damping rates with resistive and reactive feedback.

factor to overlap a larger impedance on the negative $\omega$ side than it does on the positive $\omega$ side.

One would expect that the effect of chromaticity on mode coupling would be very significant if the growth rates due to chromaticity were comparable to the growth rates due to mode coupling; this certainly seemed to be true for the $m=1$ radial modes as described above.

From the single-bunch results, one expects the features of multibunch mode coupling to be more or less unchanged, modulo a small amount of damping. Figures 5.47 and 5.48 show that the main features of multibunch mode coupling remain the same (compare figures 5.13 and 5.16). Again, this is true mainly because the damping rates from chromaticity are small compared to the growth rates due to mode coupling.

However, if the growth rates at the current of $3.159 \mathrm{~A}$ are examined (see figure 5.49), 


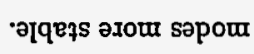

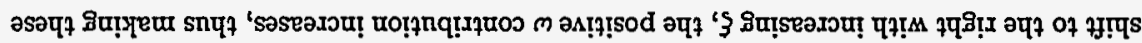

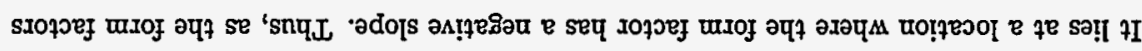

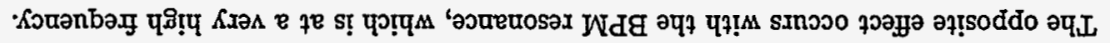

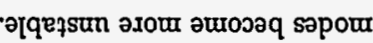

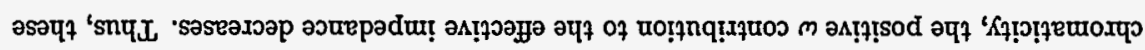

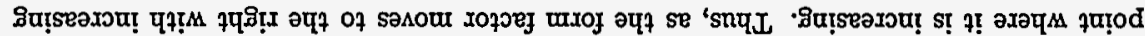

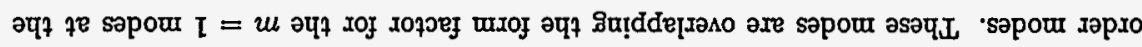

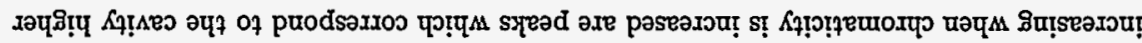

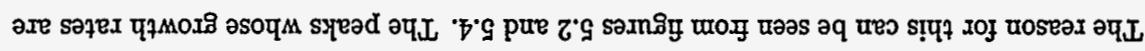

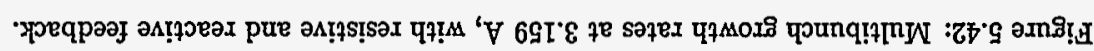

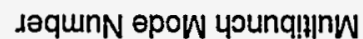

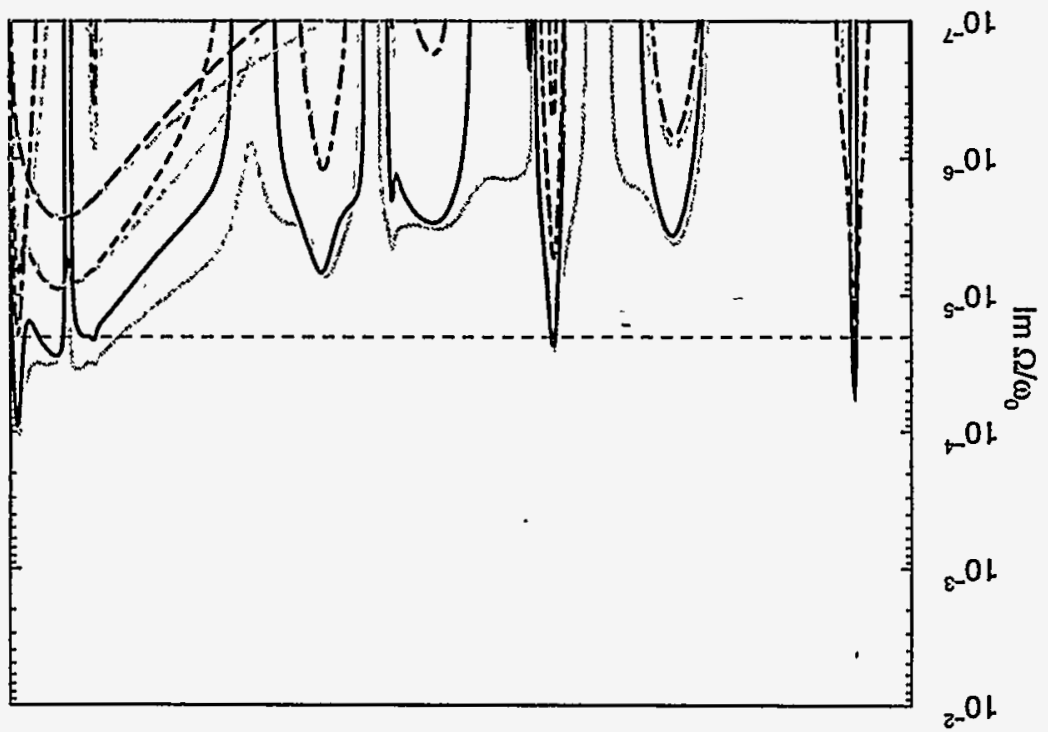

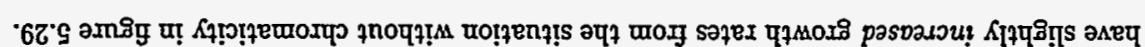

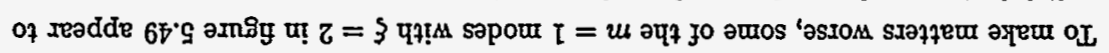

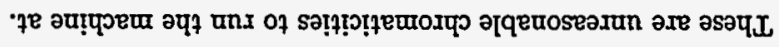

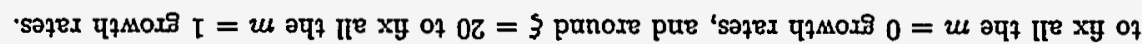

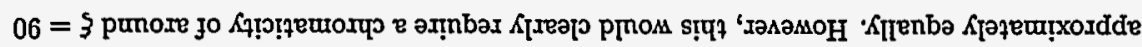

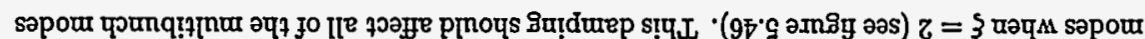

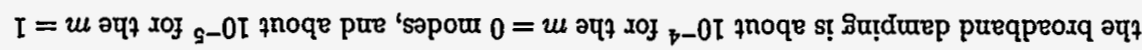

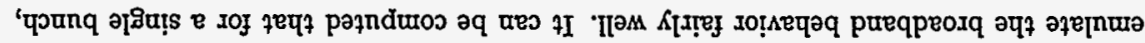

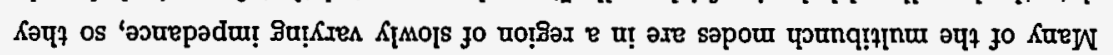

$\cdot(6 z \cdot 9$ әmริ әтеđuros)

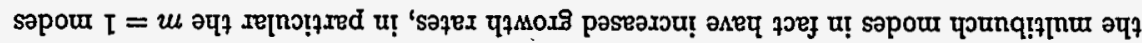

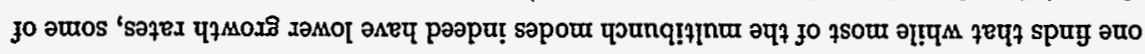

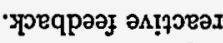

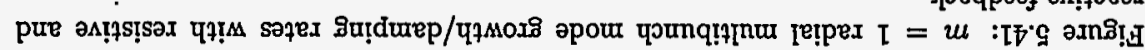

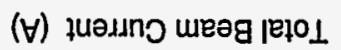

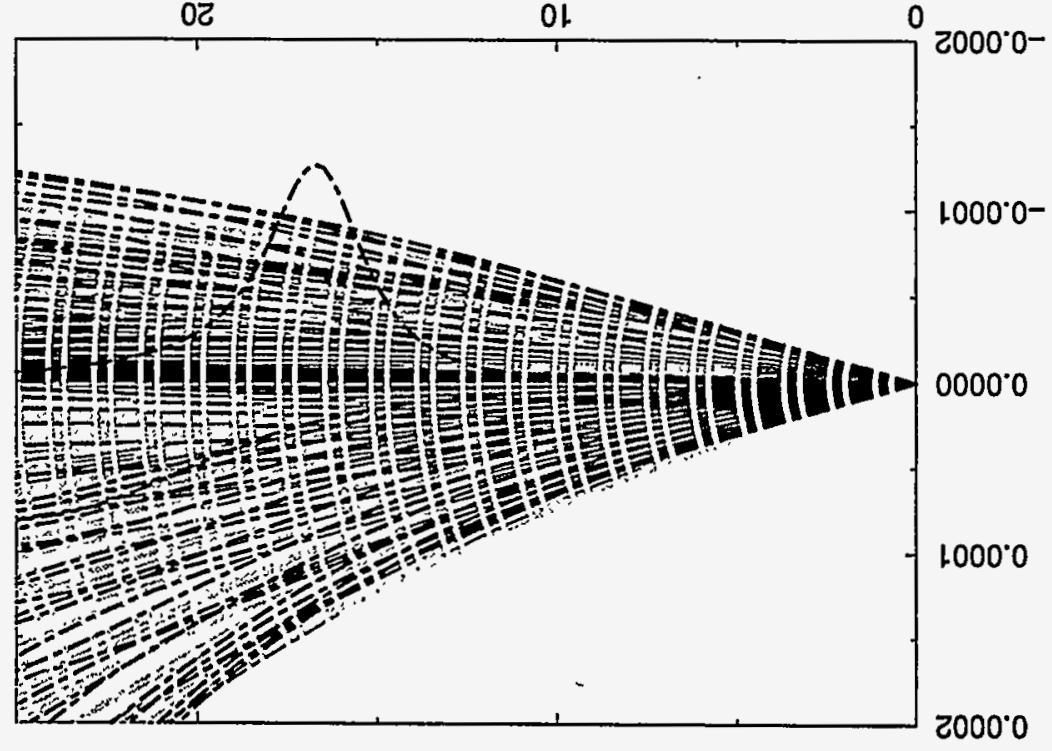

$\overline{3}$ 


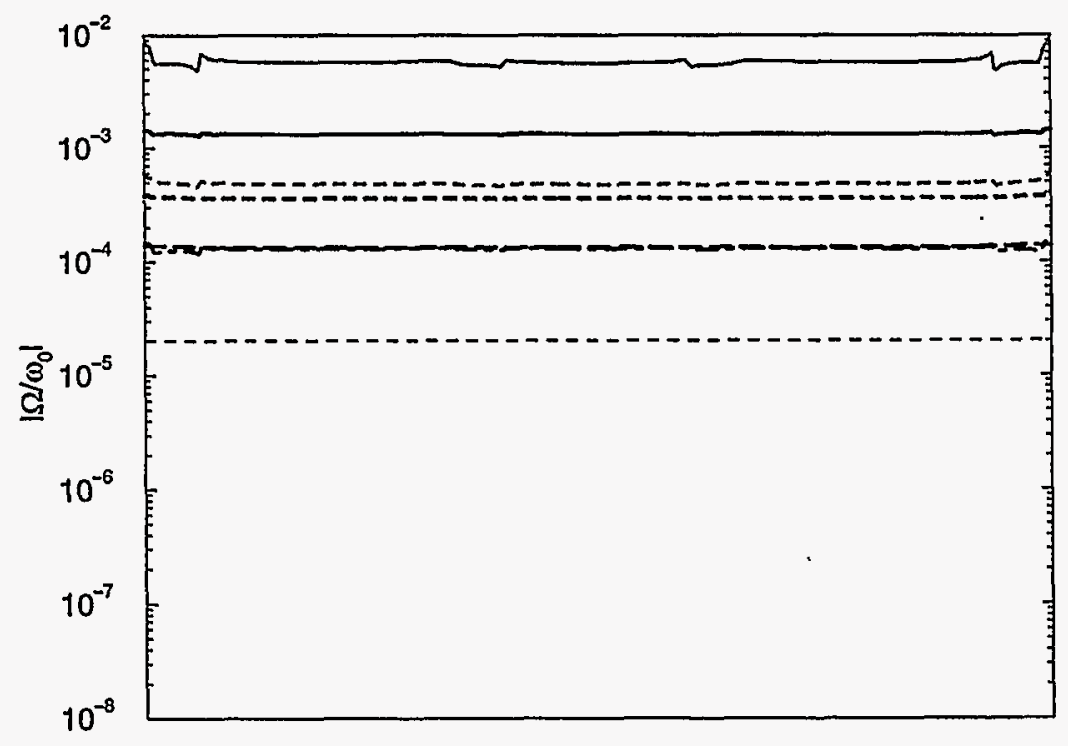

Multibunch Mode Number

Figure 5.43: Absolute value of multibunch mode shifts at $3.159 \mathrm{~A}$.

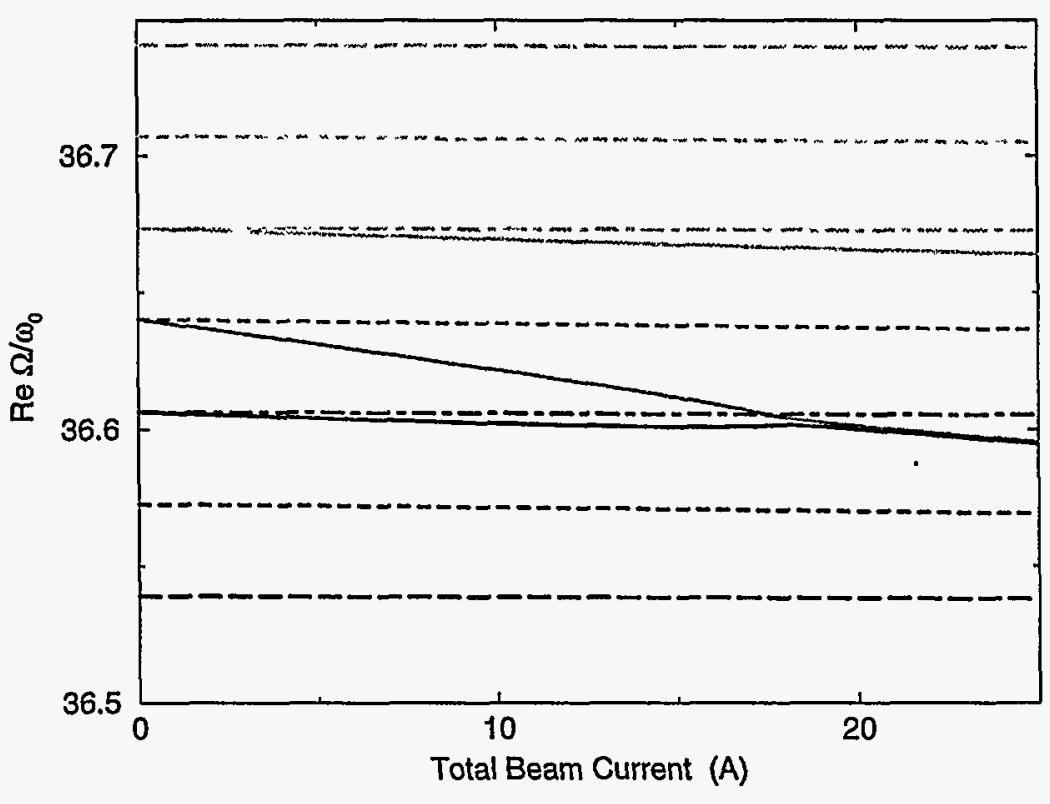

Figure 5.44: Mode frequencies for a single bunch with $\xi=2$. 


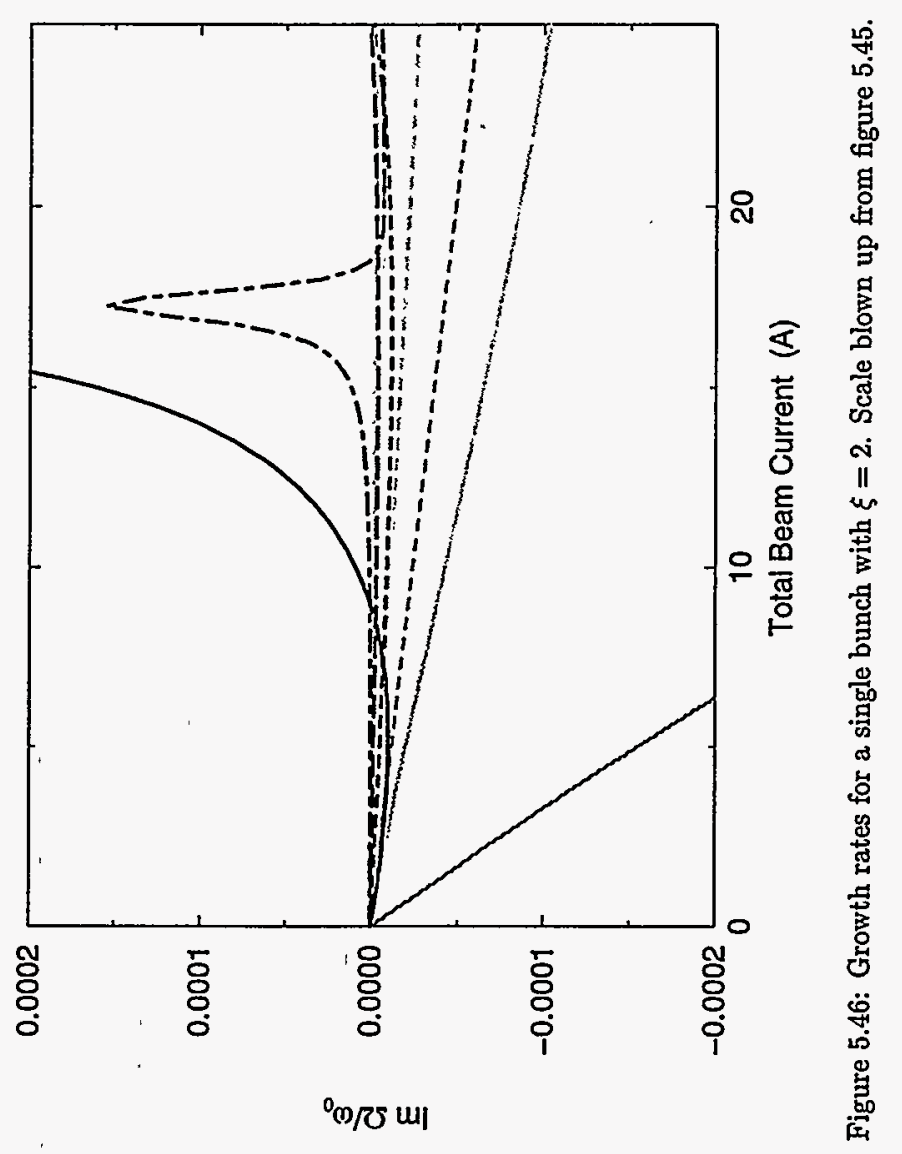

용

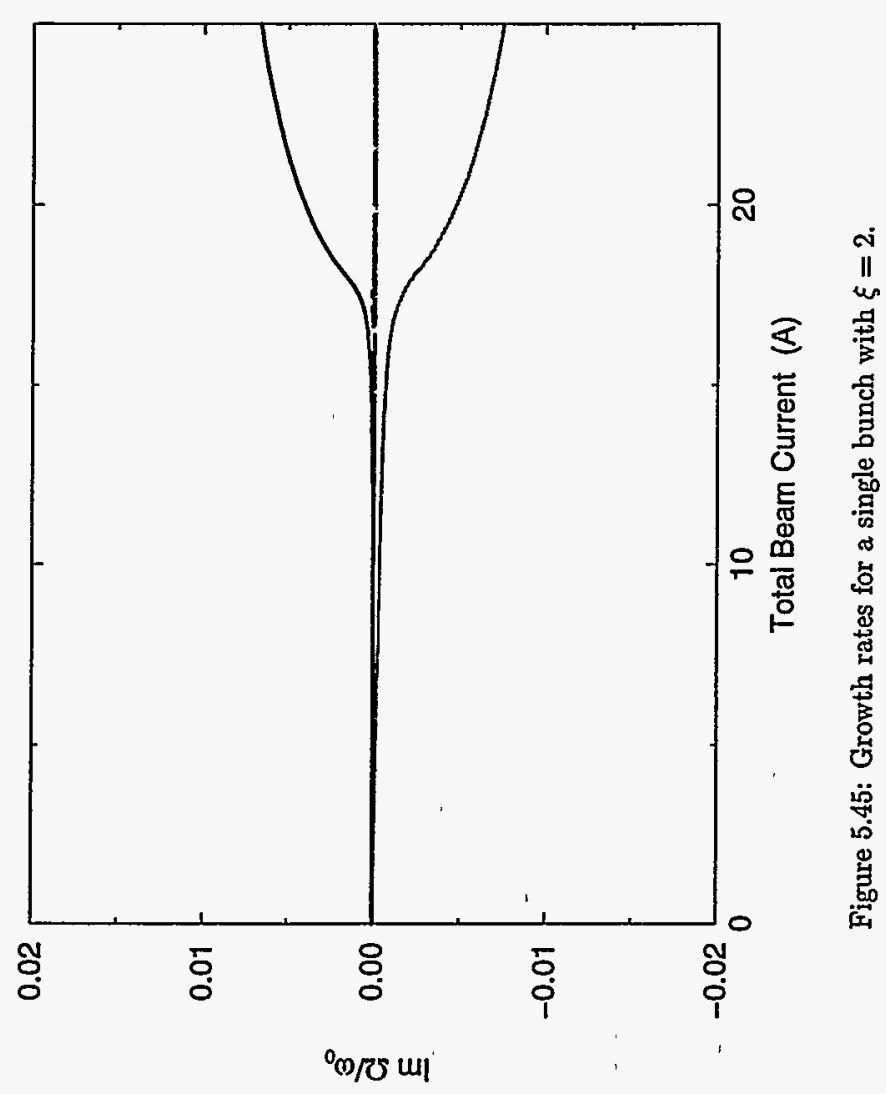




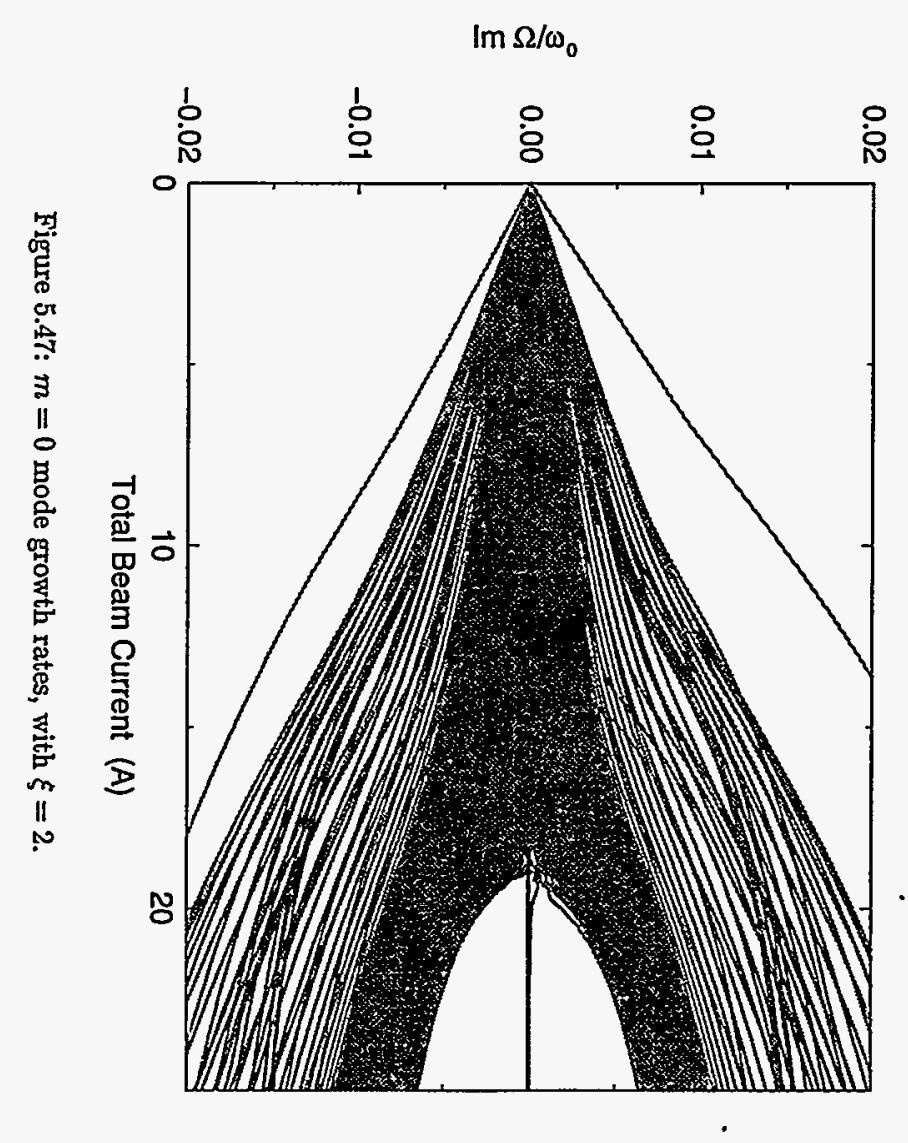

点

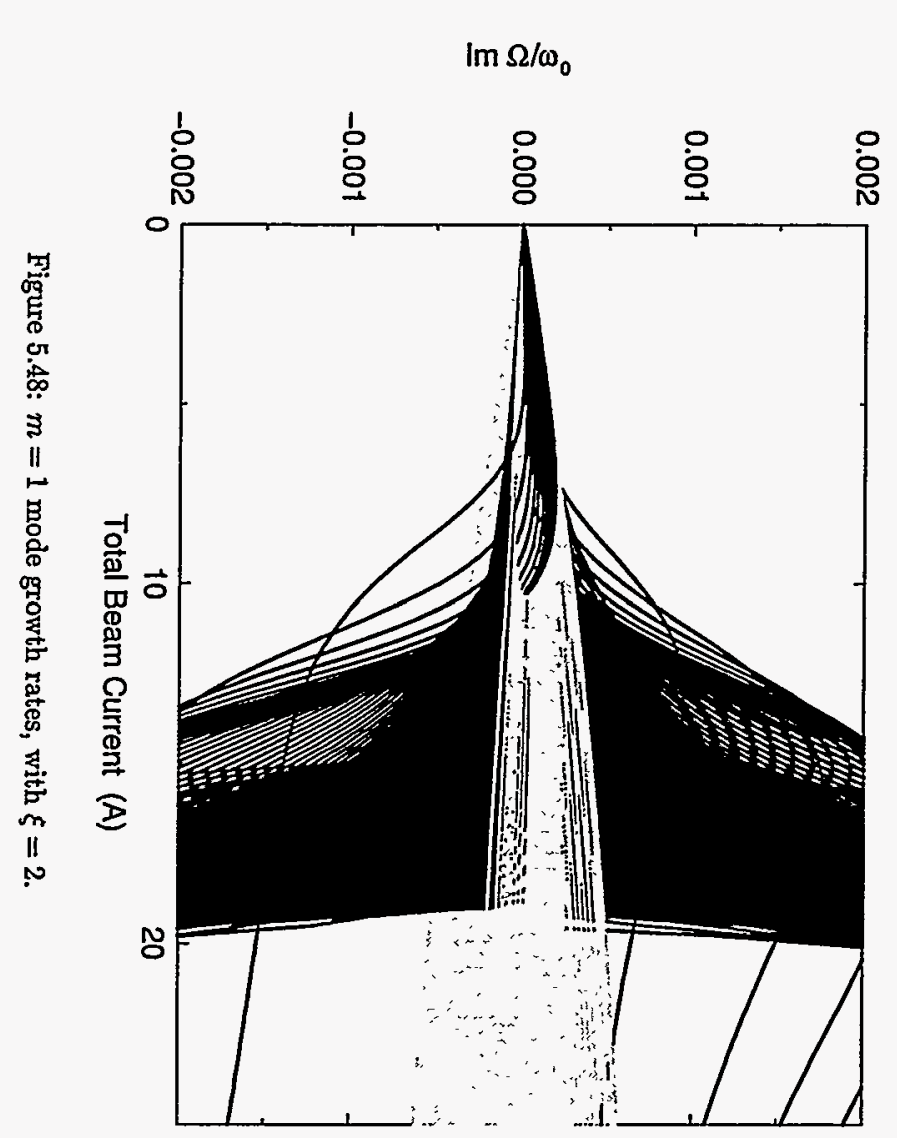

옹

究 


\section{Chapter 6}

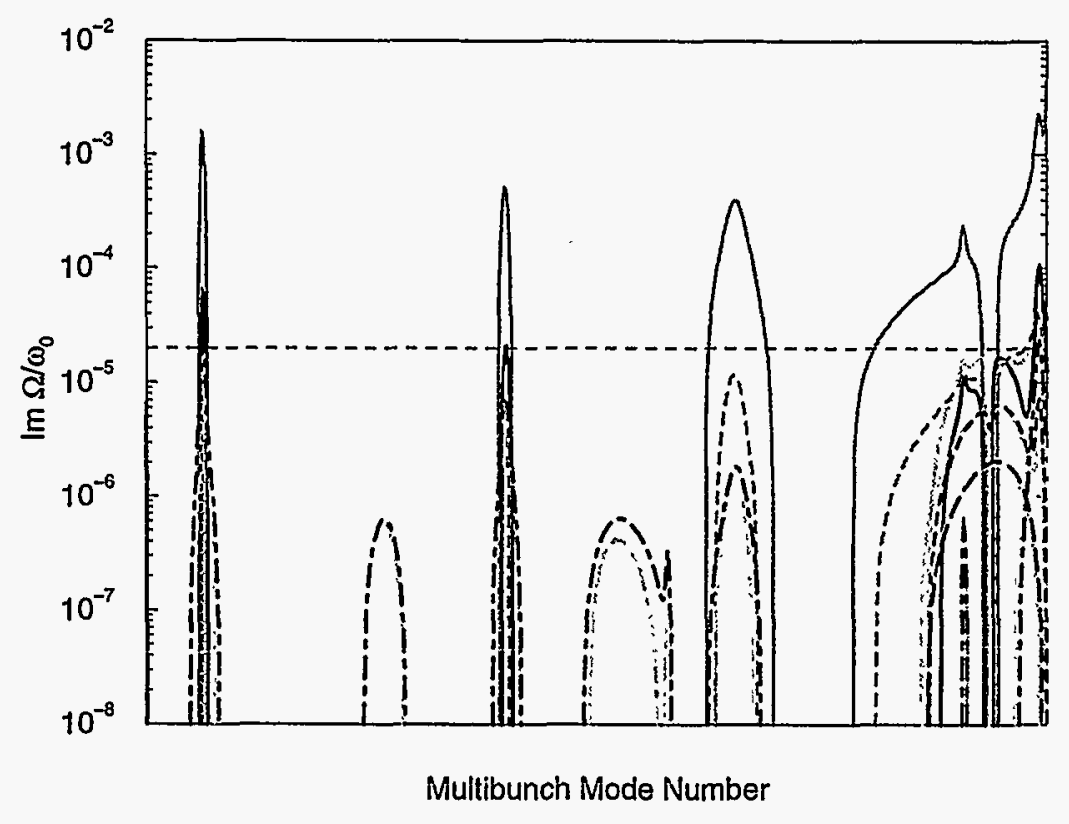

Figure 5.49: Multibunch growth rates at $3.159 \mathrm{~A}$, with $\xi=2$.

\section{Conclusion}

This dissertation has presented a method for determining the stability of a particle beam consisting of multiple bunches circulating in a storage ring. The method begins by describing the system with a Hamiltonian. The Hamiltonian is then substituted into the Vlasov equation, which determines the time evolution of a distribution of particles. Perturbations to a time-independent solution are then Fourier analyzed to determine whether they grow exponentially. If they grow faster than known damping mechanisms can account for (generally radiation damping), the system is regarded as unstable.

\subsection{Uniqueness of this Approach}

There are several new aspects to this approach. It should first be pointed out that this method follows what is generally known as the "Wang formalism," after J. M. Wang who developed it originally $[57,58,67,70]$. It has the advantage of not requiring one to guess basis functions to expand with (which is what the "Sacherer formalism" requires $[40,59,60,70]$ ): the expansion follows implicitly from the method.

This is the only approach which handles the $s$ dependence in the linear case in full detail. The factors containing $\Delta \Psi_{\tau}$ and $\Delta \Psi_{y}$ in (4.82), (4.84) and (4.85) have never appeared anywhere that I know of, and this is also the only proof that I know of for the fact that the transverse impedance should be weighted by the $\beta$-function. This is a generally accepted fact; see $[12,63,64,69]$. The correct behavior of coherent synchro-betatron resonances $[12,64]$ falls naturally out of this approach by taking $s$ Fourier modes of the weighted impedance.

The Wang formalism has only been applied to Gaussian bunches in the past. This 
dissertation shows how to apply the formalism to an arbitrary bunch distribution.

The formalism described in this dissertation outlines how to compute multibunch mode frequencies with an arbitrary Hamiltonian (possibly containing potential well distortion). It simply requires the computation of an approximate normalizing transformation, followed by integrals involving those transformations. In practice, this is of course diffcult to do for an arbitrary Hamiltonian. In this dissertation, only the linear case without dispersion has been worked out in detail. Future work will work out other more complex cases. See [46-48] for another way this can be done, at least in one dimension.

This work is the first to demonstrate the effects of multibunch mode coupling. The foundations for computing this effect were laid out by Sacherer, at least in the longitudinal direction with identical, symmetrically placed bunches [60]. This dissertation has worked out the details for both longitudinal and transverse, as well as with non-symmetric bunches. The formalism also includes radial modes, which Sacherer did not consider.

This dissertation has presented an extensive analysis of the results of multibunch mode coupling, which has not been done prior to this, other than in my own work (along with R. Ruth) [6-8].

\subsection{Summary of Results}

This dissertation has demonstrated that it is important under certain conditions to include the effects of coupling between multibunch modes when computing instability growth rates. It has been demonstrated that multibunch mode coupling gives a strong instability at about the current where the multibunch mode frequencies coincide. This current is often much lower than the single-bunch mode coupling threshold current.

However, even well below that current, the effects of mode coupling can be seen, although often less strongly. One place where it has a very significant effect is when coupling is considered between modes whose zero-current frequencies are identical. Such modes appear to grow linearly with current, but have growth rates that are significantly larger than they would be without coupling. Mode coupling also causes a nonlinear change in growth rates with current (when considering modes which have different zero-current frequencies), which can enhance some instabilities, even well below the point where the multibunch mode frequencies coincide.

A feedback system designed to damp rigid motion of the bunches will not be able to prevent multibunch mode coupling. However, such a feedback system can be used to shift the multibunch mode frequencies to make the mode coupling occur at higher currents. However, the spread in the multibunch mode frequencies limits how high a current this mode coupling threshold can be shifted to.

The low-energy ring of the PEP-II B-factory only sees minor effects from multibunch mode coupling, mainly in an enhancement of the growth rates due to the high-frequency resonance from the BPM's. If the impedance is increased by as little as a factor of 2 or 3 , however, there could be serious problems with multibunch mode coupling. The proposed feedback system can take care of the $m=0$ and $m=0$ radial mode growth rates, but the growth rates due to $m=1$ modes are problematic. It appears that a reasonable amount of chromaticity can be used to fix the growth rates due to the resonance from the BPM's, but the instabilities from resonances due to cavity higher order modes cannot be fixed in this way (in fact, they are slightly enhanced by increasing the chromaticity). Thus, these higher order modes in the cavities need to be reduced somehow.

It has been demonstrated here that multibunch mode coupling will be important for many types of machines. It is especially important for machines running with high currents and a large number of bunches. The large luminosities required for high-energy physics experiments and light sources are beginning to require such conditions. Even when a separate analysis of single-bunch mode coupling and multibunch growth rates without coupling indicates that a machine is far from a dangerous regime, the addition of coupling to the multibunch calculation will often demonstrate an instability. 


\section{Appendix A}

\section{Symplectic Transformations}

This appendix contains several results for the nonlinear dynamics underlying the description of the accelerator before collective effects are considered. Lie algebraic methods are used to perform the calculations when appropriate. For more information on these methods, see $[21,22,24,25,27,43]$.

\section{A.1 Shifts by Pure Time-Dependent Functions}

Consider the transformation

$$
z \rightarrow z+f(s)
$$

where $z$ is a 6-dimensional vector of phase space variables. This transformation will induce a transformation in the Hamiltonian $-p_{s}$. To determine that transformation, make the non-autonomous Hamiltonian autonomous by going to 8 variables; if $-p_{s}=H(z, s)$, then the new Hamiltonian is $H^{\prime}=H(z, s)+p_{s}$. The transformation (A.1) is clearly symplectic. It can be written as $z \longrightarrow e^{: \mathcal{A}} z$, where $\mathcal{A}=z^{T} J f$, and $J$ is the symplectic matrix

$$
\left[\begin{array}{cc}
0 & I \\
-I & 0
\end{array}\right] \text {. }
$$

Thus, $p_{s}$ transforms to

$$
p_{s} \rightarrow e^{: A i} z=p_{s}+z^{T} J \frac{d f}{d s}
$$

The new Hamiltonian in the 6-dimensional phase space is therefore

$$
-p_{s}^{\prime}=H(z+f(s), s)+z^{r} J \frac{d f}{d s} .
$$

\section{A.2 Linear Time-Dependent Transformations}

Consider the transformation

$$
z \rightarrow A(s) z
$$

Here $A$ is a symplectic matrix for every $s: A^{T} J A=J$, where $J$ is the symplectic matrix (A.2). As in section A.1, equation A.5 results in a change in the Hamiltonian; this change in the Hamiltonian can be computed by changing the system to an autonomous one as in section A.1. The derivative of the transformation for the autonomous system is

$$
\left[\begin{array}{ccc}
A(s) & (d A / d s) z & 0 \\
0^{T} & 1 & 0 \\
b^{T}(s) & 0 & 1
\end{array}\right] .
$$

Applying the symplectic condition to this matrix gives the result that

$$
b=A^{T} J \frac{d A}{d s} z
$$

Thus, if the original Hamiltonian is $H(z, s)$, the transformed Hamiltonian is

$$
H(A(s) z, s)+\frac{1}{2} z^{T} A^{T} J \frac{d A}{d s} z .
$$

\section{A.3 Action-Angle Variables for One-Dimensional Linear} Time-Dependent Hamiltonian

Consider the Hamiltonian

$$
\frac{1}{2} k_{p}(s) p^{2}+\frac{1}{2} k_{q}(s) q^{2}
$$

where $k_{p}$ and $k_{q}$ are periodic with period $L$. First, convert this to a two-dimensional autonomous Hamiltonian:

$$
H=\frac{1}{2} k_{p}(s) p^{2}+\frac{1}{2} k_{q}(s) q^{2}+p_{s} .
$$

Next, find two integrals of the motion for this Hamiltonian. One is $H$ itself. Another can be found by solving for the trajectory of a particle; Hamilton's equations lead to the second order differential equation

$$
\frac{d}{d s}\left(\frac{1}{k_{p}(s)} \frac{d q}{d s}\right)+k_{q}(s) q=0
$$


The solution to such a linear second order ordinary differential equation can always be written in the form

$$
q=C_{0} w(s) \cos \left[\psi(s)+C_{1}\right],
$$

where $C_{0}$ and $C_{1}$ are arbitrary constants. Plugging this solution into the differential equation (A.11) gives equations for the functions $w$ and $\psi$ :

$$
\frac{d \psi}{d s}=\frac{C_{2} k_{p}(s)}{w^{2}(s)} \quad \frac{d}{d s}\left(\frac{1}{k_{p}(s)} \frac{d w}{d s}\right)+k_{q}(s) w-\frac{C_{2}^{2} k_{p}(s)}{w^{3}}=0,
$$

where $C_{2}$ is an arbitrary constant. The function $w$ is chosen to be the periodic solution with period $L$ of the second equation in (A.13), assuming that $k_{q}$ and $k_{p}$ had the same periodicity. $C_{2}$ may be chosen to be 1 , since changing it amounts to a rescaling of $w$. The constant $C_{0}$ can now be written in terms of the phase space variables:

$$
C_{0}^{2}=\left(\frac{q}{w(s)}\right)^{2}+\left(p w(s)-\frac{d w}{d s} \frac{q}{k_{p}(s)}\right)^{2}
$$

Clearly $C_{0}^{2}$ is an integral of the motion.

Now that there are two constants of integration, the action variables can be computed in terms of these constants of integration. Action variables are computed by integrating $p d q+p_{s} d s$ around a closed curve on a surface where the integrals of the motion are constant [3]. The first action variable, which will be called $J_{q}$, is computed by integrating around a curve of constant $s$. The result is that $J_{q}=C_{0}^{2} / 2$. The second action variable, $J_{s}$, is computed by integrating around a curve where $q=0$. The curve encompasses one period in $s$. The result is that $J_{s}=H L / 2 \pi-J_{q} \mu / 2 \pi$, where

$$
\mu=\int_{0}^{L} \frac{k_{p}(s)}{w^{2}(s)} d s
$$

Thus, the Hamiltonian can be written in terms of the action-angle variables as

$$
H=\frac{2 \pi J_{s}}{L}+\frac{\mu J_{q}}{L} \text {. }
$$

The angle variables corresponding to $J_{q}$ and $J_{s}$ can be computed by finding a mixedvariable generating function for the symplectic transformation to action-angle variables. The generating function is of the form $F\left(q, s, J_{q}, J_{s}\right)$, and the transformation is defined by the relations

$$
p=\frac{\partial F}{\partial q} \quad p_{s}=\frac{\partial F}{\partial s} \quad \theta_{q}=\frac{\partial F}{\partial J_{q}} \quad \theta_{s}=\frac{\partial F}{\partial J_{s}} .
$$

Integrating the first two relations gives the generating function $F$; the last two relations then define $\theta_{q}$ and $\theta_{s}$. These turn out to be defined through

$$
q=\sqrt{2 J_{q}} w(s) \cos \left[\theta_{q}+\psi(s)-\frac{\mu s}{L}\right] \quad \theta_{s}=\frac{2 \pi s}{L},
$$

where

$$
\psi(s)=\int_{0}^{s} \frac{k_{p}(s)}{w^{2}(s)} d s
$$

Now change variables to $s=\theta_{s} L / 2 \pi$ and $p_{s}=2 \pi J_{s} / L$. The resulting Hamiltonian can now be converted back into a one-dimensional Hamiltonian with $s$ as the independent variable: $H=\mu J_{q} / L$.

Finally, $p$ can be obtained by taking the $s$ derivative of $q$; the result is

$$
p=\frac{\sqrt{2 J_{q}}}{k_{p}} \frac{d w}{d s} \cos \left[\theta_{q}+\psi(s)-\frac{\mu s}{L}\right]-\frac{\sqrt{2 J_{q}}}{w(s)} \sin \left[\theta_{q}+\psi(s)-\frac{\mu s}{L}\right] .
$$

The matrix describing a one-period symplectic transformation (assuming the map is stable) for this system can be written as $[19,21]$

$$
\left[\begin{array}{cc}
\cos \mu+\alpha \sin \mu & \beta \sin \mu \\
-\frac{1+\alpha^{2}}{\beta} \sin \mu & \cos \mu-\alpha \sin \mu
\end{array}\right] .
$$

Computing the one-period map from equations (A.18) and (A.20), the functions $\beta(s)$ and $\alpha(s)$ can be identified as

$$
\beta(s)=w^{2}(s) \quad \alpha(s)=-\frac{w(s)}{k_{p}(s)} \frac{d w}{d s} .
$$

It is useful to define the beam emittance $\epsilon_{q}$ of a beam distribution as the average of $J_{q}$ over the distribution $[10,68]$.

\section{A.4 Map for a One-Dimensional Single-Kick Hamiltonian}

Consider a Hamiltonian of the form

$$
H=\frac{1}{2} k_{p}(s) p^{2}+\frac{1}{2} k_{q} \delta_{L}\left(s-s_{q}\right) q^{2},
$$


where $k_{p}$ is periodic with period $L$. While the method of section A.3 could be applied to this Hamiltonian, it is more convenient to start with the one-period map in this case. The one-period map starting from a point $s$ and returning to that same point is described by the matrix

$$
\left[\begin{array}{cc}
1-k_{q}\left[\hat{K}_{p}-K_{p}(s)\right] & \hat{K}_{p}-k_{q} K_{p}(s)\left[\hat{K}_{p}-K_{p}(s)\right] \\
-k_{q} & 1-k_{q} K_{p}(s)
\end{array}\right]
$$

where

$$
K_{p}(s)=\int_{s_{q}}^{s} k_{p}\left(s^{\prime}\right) d s^{\prime}, s_{q} \leq s \leq s_{q}+L \quad K_{p}(s+L)=K_{p}(s) .
$$

Define $\hat{K}_{p} \equiv K_{p}\left(s_{q}+L\right)$.

Comparing the matrix (A.24) to the matrix (A.21), the quantities $\mu, \alpha(s)$, and $\beta(s)$ can be read off:

$$
\begin{gathered}
\mu=2 \operatorname{sign} k_{q} \sin ^{-1} \frac{\sqrt{k_{q} \hat{K}_{p}}}{2} \\
\alpha(s)=\frac{\operatorname{sign} k_{q}}{2} \sqrt{\frac{k_{q} \hat{K}_{p}}{1-k_{q} \hat{K}_{p} / 4} \frac{\hat{K}_{p}-2 K_{p}(s)}{\hat{K}_{p}}} \\
\beta(s)=\frac{\hat{K}_{p}}{\sin \mu}\left[1-k_{q} \hat{K}_{p} \frac{K_{p}(s)\left[\hat{K}_{p}-K_{p}(s)\right]}{\hat{K}_{p}^{2}}\right] \\
=\sqrt{\frac{\hat{K}_{p}}{k_{q}}} \frac{1}{\sqrt{1-k_{q} \hat{K}_{p} / 4}}\left[1-k_{q} \hat{K}_{p} \frac{K_{p}(s)\left[\hat{K}_{p}-K_{p}(s)\right]}{\hat{K}_{p}^{2}}\right] .
\end{gathered}
$$

$\alpha$ and $\beta$ satisfy the inequalities

$$
|\alpha(s)| \leq \sin \frac{\mu}{2} \tan \frac{\mu}{2} \quad \frac{\beta(0)-\beta(s)}{\beta(0)} \leq \sin ^{2} \frac{\mu}{2} .
$$

Note that $\beta(0)$ is the maximum value of $\beta$, assuming that $K_{p}(s)$ is monotonically increasing with $s$. Thus, if $\mu$ is small, then $\alpha$ is nearly zero, and $\beta$ is nearly constant. Combining equations (A.19) and (A.22) with equation (A.28), $\psi(s)$ can also be determined:

$$
\psi(s)=\tan ^{-1}\left[\sqrt{\frac{k_{q} \hat{K}_{p}}{1-k_{q} \hat{K}_{p} / 4}}\left(\frac{K_{p}(s)}{\hat{K}_{p}}-\frac{1}{2}\right)\right]+\tan ^{-1}\left[\frac{1}{2} \sqrt{\frac{k_{q} \hat{K}_{p}}{1-k_{q} \hat{K}_{p} / 4}}\right]
$$

Thus, $q$ and $p$ can be written in terms of the action $J_{q}$ and angle $\theta_{q}$ using equations (A.18), (A.20), and (A.22):

$$
\begin{gathered}
q=\sqrt{2 J_{q} \beta(s)} \cos \left(\theta_{q}+\psi(s)-\frac{\mu s}{L}\right) \\
p=-\sqrt{\frac{2 J_{q}}{\beta(s)}}\left[\sin \left(\theta_{q}+\psi(s)-\frac{\mu s}{L}\right)+\alpha(s) \cos \left(\theta_{q}+\psi(s)-\frac{\mu s}{L}\right)\right] .
\end{gathered}
$$

\section{A.5 Nonlinear Normalization}

Begin with a Hamiltonian of the form

$$
H=H_{0}+H_{1}+p_{s},
$$

where $H_{0}$ is already in normal form. The exact definition of "normal form" varies with the problem. The goal of the normalization process is to make a change of variables so that at least the lowest order parts of $H_{1}$, when combined with $H_{0}$, are in normal form.

Take $H_{1}$ to be a perturbation to $H_{0}$. Then the goal is to choose an $\mathcal{A}$ such that

$$
\frac{\partial \mathcal{A}}{\partial s}+\left[\mathcal{A}, \hat{H}_{0}\right]+\hat{H}_{1}=\Delta H_{0}
$$

where $H_{0}+\Delta H_{0}$ is in normal form. $\hat{H}_{0}$ and $\hat{H}_{1}$ are lower order parts of $H_{0}$ and $H_{1}$ respectively. Exactly how much of $H_{0}$ and $H_{1}$ they contain determines the behavior of the perturbation theory. Since this dissertation deals with rings, $\mathcal{A}$ should be periodic in $s$ with period $L$. This $\mathcal{A}$ defines a Lie transformation $e^{: A \text { : }}$ which defines a change of variables to "more normal" coordinates. The Hamiltonian in the new variables will be $H_{0}+\Delta H_{0}$ plus terms which are higher order than $H_{1}$.

\section{A.5.1 Action-Angle Lowest Order Transformation}

Say that $H_{0}=H_{0}(J)$, and the desired normal form also takes on this form. Then, defining $\omega(J)=\partial H_{0} / \partial J$, equation (A.34) becomes

$$
\frac{\partial \mathcal{A}}{\partial s}+\omega(J) \cdot \frac{\partial \mathcal{A}}{\partial \theta}+H_{1}(\theta, J, s)=\Delta H_{0}(J) .
$$

Begin by Fourier analyzing $H_{1}$ and $\mathcal{A}$ in $\theta$ :

$$
\begin{aligned}
\mathcal{A}(\theta, J, s) & =\sum_{m} a_{m}(J, s) e^{i m \cdot \theta} \\
H_{1}(\theta, J, s) & =\sum_{m} h_{m}(J, s) e^{i m \cdot \theta} .
\end{aligned}
$$


Then equation (A.35) becomes

$$
\frac{\partial a_{m}}{\partial s}+i m \cdot \omega(J) a_{m}(J, s)+h_{m}(J, s)=\delta_{m 0} \Delta H_{0}(J) .
$$

There are two cases for the solution of equation (A.38). First, consider $m=0$. This case gives

$$
\frac{\partial a_{0}}{\partial s}+h_{0}(J, s)=\Delta H_{0}(J)
$$

Clearly

$$
\Delta H_{0}(J)=\frac{1}{L} \int_{0}^{L} h_{0}(J, s) d s,
$$

since the periodicity of $a_{0}$ will give the derivative of $a_{0}$ an average over $s$ of zero. It is easy to verify that the resulting $a_{0}$ is

$$
a_{0}(J, s)=-\frac{1}{L} \int_{s}^{s+L}\left(s^{\prime}-s-\frac{L}{2}\right) h_{0}\left(J, s^{\prime}\right) d s^{\prime}=-\frac{1}{L} \int_{-L / 2}^{L / 2} s^{\prime} h_{0}\left(J, s^{\prime}+s+L / 2\right) d s^{\prime} .
$$

Next, consider the case where $m \neq 0$. Then equation (A.38) becomes

$$
\frac{\partial a_{m}}{\partial s}+i m \cdot \omega(J) a_{m}(J, s)=-h_{m}(J, s) .
$$

This has the periodic solution

$$
\begin{aligned}
a_{m}(J, s) & =\frac{1}{1-e^{i m \cdot \omega(J) L}} \int_{s}^{s+L} h_{m}\left(J, s^{\prime}\right) e^{i m \cdot \omega(J)\left(s^{\prime}-s\right)} d s^{\prime} \\
& =-\frac{i}{2} \csc \frac{m \cdot \omega(J) L}{2} \int_{s}^{s+L} h_{m}\left(J, s^{\prime}\right) e^{i m \cdot \omega(J)\left(s^{\prime}-s-L / 2\right)} d s^{\prime} \\
& =-\frac{i}{2} \csc \frac{m \cdot \omega(J) L}{2} \int_{-L / 2}^{L / 2} h_{m}\left(J, s^{\prime}+s+L / 2\right) e^{i m \cdot \omega(J) s^{\prime}} d s^{\prime}
\end{aligned}
$$

All this suffices to specify $\mathcal{A}$ and $\Delta H_{0}(J)$.

\section{A.6 Synchrotron Tune Shift with Amplitude}

Begin with the Hamiltonian for the synchrotron motion of

$$
-\frac{1}{2}\left[h(s) \eta(s)-\frac{1}{\gamma_{0}^{2}}\right]+\frac{e \hat{V}}{p_{0} \omega_{\mathrm{rf}}} \delta_{L}\left(s-s_{\mathrm{rf}}\right) \cos \left(\omega_{\mathrm{rf}} \tau+\phi_{\mathrm{rf}}\right),
$$

as described in sections 3.2.3 and 3.2.4.

The effect of energy loss can be added to this model, although it cannot be described by a Hamiltonian. If a particle following the ideal $\tau=0$ trajectory receives an energy loss of $\Delta E$ per turn, then the phase of the $\mathrm{rf}$ is adjusted so as to compensate this loss. Thus, $e \hat{V} \sin \phi_{\mathrm{rf}}=-\Delta E$. The energy loss is taken to be independent of the particle trajectory, which allows the computation to be formulated in terms of a symplectic map. To simplify the calculation, the energy loss is considered to occur immediately before the rf cavity.

As seen above in section A.4, it is simpler to work with the Hamiltonian as a map. Writing the map in terms of Lie operators, the one-turn map just after the rf cavity is

$$
\begin{aligned}
M= & \exp \left(\frac{L \eta_{C}}{2 \beta_{0}^{2} c^{2}} \delta^{2}\right) \exp \left(\frac{e \hat{V} \omega_{\mathrm{rf}}}{2 p_{0}} \cos \phi_{\mathrm{rf}} \tau^{2}\right) \\
& \exp \left(\frac{e \hat{V}}{p_{0} \omega_{\mathrm{rf}}}\left[\cos \phi_{\mathrm{rf}}-\omega_{\mathrm{rf}} \tau \sin \phi_{\mathrm{rf}}-\frac{1}{2} \omega_{\mathrm{rf}}^{2} \tau^{2} \cos \phi_{\mathrm{rf}}-\cos \left(\omega_{\mathrm{rf}} \tau+\phi_{\mathrm{rf}}\right)\right]\right) .
\end{aligned}
$$

The linear part of $M$ can be normalized using the transformations described in section A.4, with the definitions of equations (4.31)-(4.33). The transformation to normalized coordinates can be written as the matrix

$$
A=\left[\begin{array}{cc}
\sqrt{\beta_{\tau}\left(s_{\mathrm{rf}}\right)} & 0 \\
-\alpha_{\tau}\left(s_{\mathrm{rf}}\right) / \sqrt{\beta_{\tau}\left(s_{\mathrm{rf}}\right)} & 1 / \sqrt{\beta_{\tau}\left(s_{\mathrm{rf}}\right)}
\end{array}\right] .
$$

Thus,

$$
\begin{aligned}
M=A \exp \left(-\frac{\mu_{\tau}}{2}\left(\tau^{2}+\delta^{2}\right)\right) & \exp \left(\frac { e \hat { V } } { p _ { 0 } \omega _ { \mathrm { rf } } } \left[\cos \phi_{\mathrm{rf}}-\omega_{\mathrm{rf}} \sqrt{\beta_{\tau}\left(s_{\mathrm{rf}}\right)} \tau \sin \phi_{\mathrm{rf}}\right.\right. \\
& \left.\left.-\frac{1}{2} \omega_{\mathrm{rf}}^{2} \beta_{\tau}\left(s_{\mathrm{rf}}\right) \tau^{2} \cos \phi_{\mathrm{rf}}-\cos \left(\omega_{\mathrm{rf}} \sqrt{\beta_{\tau}\left(s_{\mathrm{rf}}\right)} \tau+\phi_{\mathrm{rf}}\right)\right]\right) A^{-1} .
\end{aligned}
$$

Note that $\beta_{\tau}\left(s_{\mathrm{rf}}\right)=-\eta_{C} L / \beta_{0}^{2} c^{2} \sin \mu_{\tau}$, using equations (4.32) and (A.28).

Expanding the second exponential in (A.49) to fourth order gives

$$
M=A R e^{f_{3}+f_{4}} A^{-1}
$$


where

$$
\begin{gathered}
R=\exp \left(-\frac{\mu_{\tau}}{2}\left(\tau^{2}+\delta^{2}\right)\right) \\
f_{3}=-\frac{1}{6} \sin \phi_{\mathrm{rf}} \frac{e \hat{V}}{\omega_{\mathrm{rf}} p_{0}}\left(\frac{\omega_{\mathrm{rf}}}{\beta_{0} c}\right)^{3}\left(\eta_{C} L \csc \mu_{\tau}\right)^{3 / 2} \tau^{3}=\frac{1}{3} \tan \phi_{\mathrm{rf}} \frac{\omega_{\mathrm{rf}}}{\beta_{0} c} \sqrt{-\eta_{C} L \csc \mu_{\tau}} \tan \frac{\mu_{\tau}}{2} \tau^{3} \\
f_{4}=-\frac{1}{24} \cos \phi_{\mathrm{rf}} \frac{e \hat{V}}{\omega_{\mathrm{rf}} p_{0}}\left(\frac{\omega_{\mathrm{rf}}}{\beta_{0} c}\right)^{4}\left(\eta_{C} L \csc \mu_{\tau}\right)^{2} \tau^{4}=-\frac{1}{24}\left(\frac{\omega_{\mathrm{rf}}}{\beta_{0} c}\right)^{2} \eta_{C} L \sec ^{2} \frac{\mu_{\tau}}{2} \tau^{4}
\end{gathered}
$$

The normalization process amounts to writing

$$
R e^{f_{3}+f_{4}}=e^{a_{3}+a_{4}} R e^{h_{4}} e^{-a_{3}-a_{4}},
$$

where $a_{3}$ and $a_{4}$ are third and fourth order homogeneous polynomials respectively, and $h_{4}$ has a fourth order term which commutes with $R$ plus higher order terms. Thus, equating terms of equal order, equation (A.54) becomes equivalent to

$$
\begin{gathered}
a_{3}=\left(R^{-1}-1\right)^{-1} f_{3} \\
h_{4}=P_{4}\left(f_{4}+\left[R^{-1} a_{3}, a_{3}\right] / 2\right) \\
a_{4}=\left(R^{-1}-1\right)^{-1}\left(f_{4}+\left[R^{-1} a_{3}, a_{3}\right] / 2-h_{4}\right),
\end{gathered}
$$

where $P_{4}$ is the operator that projects out the component of $f_{4}$ which commutes with $R$. The higher order terms in $h_{4}$ have been ignored. In equations (A.55)-(A.57), $R$ and $P_{4}$ are treated as operators operating on the polynomials.

Define the action-angle coordinates $J$ and $\theta$ to be $\tau=\sqrt{2 J} \cos \theta$ and $\delta=-\sqrt{2 J} \sin \theta$. Then $R=e^{-\mu_{\tau} J}$, and the eigenfunctions of $R$ are clearly $f(J) e^{i k \theta}$, where $f$ is an arbitrary function of $J$. The projection operator $P_{4}$ simply picks out the term with $k=0$ in the Fourier series in $\theta$ for a fourth order homogeneous polynomial in $\tau$ and $\delta$.

With the change of variables to $J$ and $\theta, a_{3}$ is

$$
\begin{aligned}
\frac{1}{24} \tan \frac{\mu_{\tau}}{2} \tan \phi_{\mathrm{r} f} \frac{\omega_{\mathrm{rf}}}{\beta_{0 c}} \sqrt{-\eta_{C} L \csc \mu_{\tau}}(2 J)^{3 / 2} \\
\quad\left(\frac{e^{3 i \theta}}{e^{-3 i \mu_{\tau}-1}}+\frac{3 e^{i \theta}}{e^{-i \mu_{\tau}-1}}+\frac{3 e^{-i \theta}}{e^{i \mu_{\tau}-1}}+\frac{e^{-3 i \theta}}{e^{3 i \mu_{\tau}}-1}\right) .
\end{aligned}
$$

This can be then used to compute the $\theta$-independent component of $\left[R^{-1} a_{3}, a_{3}\right] / 2$ :

$$
-\frac{1}{128} \tan ^{2} \phi_{\mathrm{rf}} \sec ^{2} \frac{\mu_{\tau}}{2} \csc \mu_{\tau}\left(\frac{\omega_{\mathrm{rf}}}{\beta_{0} c}\right)^{2} \eta_{C} L(2 J)^{2}\left(3+\frac{\tan \mu_{\tau} / 2}{\tan 3 \mu_{\tau} / 2}\right)
$$

The $\theta$-independent component of $f_{4}$ is

$$
-\frac{1}{64} \sec ^{2} \frac{\mu_{\tau}}{2}\left(\frac{\omega_{\mathrm{rf}}}{\beta_{0} c}\right)^{2} \eta_{C} L(2 J)^{2} .
$$

Combining these two, $h_{4}$ is

$$
-\frac{1}{128} \sec ^{2} \frac{\mu_{\tau}}{2}\left(\frac{\omega_{\mathrm{rf}}}{\beta_{0} c}\right)^{2} \eta_{C} L(2 J)^{2}\left[2+\tan ^{2} \phi_{\mathrm{rf}}\left(3+\frac{\tan \mu_{\tau} / 2}{\tan 3 \mu_{\tau} / 2}\right)\right]
$$

Thus, the tune, to first order in $J$, is

$$
\nu=\nu_{\tau}+\frac{1}{32 \pi} \sec ^{2} \frac{\mu_{\tau}}{2}\left(\frac{\omega_{\mathrm{rf}}}{\beta_{0} c}\right)^{2} \eta_{C} L\left[2+\tan ^{2} \phi_{\mathrm{rf}}\left(3+\frac{\tan \mu_{\tau} / 2}{\tan 3 \mu_{\tau} / 2}\right)\right] J
$$

Note that since $\nu_{\tau}$ is generally negative, the absolute value of the tune is decreasing with increasing $J$. The emittance can be written as $-\sigma_{\ell}^{2} \sin \mu_{\tau} / \eta_{C} L$, and thus the tune shift at this emittance is

$$
\Delta \nu_{\tau}=-\frac{1}{16 \pi} \tan \frac{\mu_{\tau}}{2}\left(\frac{\sigma_{\ell} \omega_{\mathrm{rf}}}{\beta_{0} c}\right)^{2}\left[2+\tan ^{2} \phi_{\mathrm{rf}}\left(3+\frac{\tan \mu_{\tau} / 2}{\tan 3 \mu_{\tau} / 2}\right)\right] .
$$




\section{Appendix B}

\section{Action-Dependent Distribution}

\section{Function}

Assume that the action is given as a function of $q$ and $p$ as

$$
J=\frac{1}{2} \frac{1+\alpha^{2}}{\beta} q^{2}+\alpha q p+\frac{1}{2} \beta p^{2}
$$

as described in appendix A.3. Next, consider a distribution function $\Psi$ which is only a function of $J$. Assume that the distribution function $\rho$ in $q$ is known; i.e.,

$$
\rho(q)=\int_{-\infty}^{\infty} \Psi\left(\frac{1}{2} \frac{1+\alpha^{2}}{\beta} q^{2}+\alpha q p+\frac{1}{2} \beta p^{2}\right) d p .
$$

This appendix describes how to determine $\Psi$ from $\rho$.

The first step is to Fourier transform both sides of equation (B.2) over $q$ :

$$
\int_{-\infty}^{\infty} d q \rho(q) e^{i \omega q}=2 \pi \int_{0}^{\infty} d J \Psi(J) J_{0}(\omega \sqrt{2 J \beta})
$$

where $J_{0}$ is a Bessel function [1]. The right hand side is just the Hankel transform of $\Psi$; the Hankel transform is easily inverted to give

$$
\Psi(J)=\frac{\beta}{2 \pi} \int_{0}^{\infty} d \omega \int_{-\infty}^{\infty} d q \omega J_{0}(\omega \sqrt{2 J \beta}) \rho(q) e^{i \omega q}
$$

First, integrate the $q$ integral by parts:

$$
\Psi(J)=\frac{i \beta}{2 \pi} \int_{0}^{\infty} d \omega \int_{-\infty}^{\infty} d q J_{0}(\omega \sqrt{2 J \beta}) \rho^{\prime}(q) e^{i \omega q}
$$

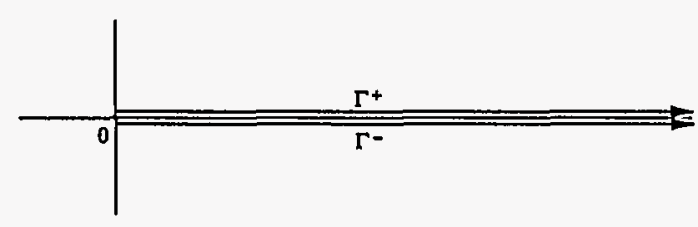

Figure B.1: Contours of integration for equation (B.7)

where $\rho^{\prime}$ denotes the derivative of $\rho$. The Bessel function can be expressed as an integral, so that

$$
\begin{aligned}
\Psi(J) & =\frac{i \beta}{\pi^{2}} \int_{0}^{\infty} d \omega \int_{-\infty}^{\infty} d q \int_{0}^{\infty} d t \sin (\omega \sqrt{2 J \beta} \cosh t) \rho^{\prime}(q) e^{i \omega q} \\
& =\frac{\beta}{2 \pi^{2}} \int_{0}^{\infty} d \omega \int_{-\infty}^{\infty} d q \int_{0}^{\infty} d t\left(e^{i \omega \sqrt{2 J \beta} \cosh t}-e^{-i \omega \sqrt{2 J \beta} \cosh t}\right) \rho^{\prime}(q) e^{i \omega q} .
\end{aligned}
$$

To be able to switch the order of integration and perform the $\omega$ integral, the Fubini theorem [54] requires that one of the iterated integrals be absolutely integrable, and then the remaining integrals give a finite result. To satisfy this condition, distort the contour for the $t$ integral up or down in the complex plane depending on which of the exponential terms are being dealt with. The exponential in question will then go to zero for large $\omega$, meeting the criteria of absolute integrability. The resulting integral can be written as

$$
\Psi(J)=\frac{\beta}{2 \pi^{2}} \int_{0}^{\infty} d \omega \int_{-\infty}^{\infty} d q\left[\int_{\Gamma^{+}} d t e^{i \omega \sqrt{2 J \beta} \cosh t}-\int_{\Gamma^{-}} d t e^{-i \omega \sqrt{2 J \beta} \cosh t}\right] \rho^{\prime}(q) e^{i \omega q},
$$

where $\Gamma^{+}$and $\Gamma^{-}$are shown in figure B.1. The $\omega$ integral may now be performed. Taking advantage of the fact that $\rho(q)$ must be even in $q$, the order of $q$ integration for the first term can be changed. The result is

$$
\Psi(J)=\frac{i \beta}{2 \pi^{2}} \int_{\Gamma^{-}-\Gamma^{+}} d t \int_{-\infty}^{\infty} d q \frac{\rho^{\prime}(q)}{\sqrt{2 J \beta} \cosh t-q} .
$$

Since the integrand decreases extremely rapidly to zero as $t \rightarrow \infty$, the $t$ integral can be closed near infinity. Thus, the residue theorem can be applied to evaluate this integral, giving

$$
\Psi(J)=-\frac{\beta}{\pi} \int_{\sqrt{2 J \beta}}^{\infty} d q \frac{\rho^{\prime}(q)}{\sqrt{q^{2}-2 J \beta}}=-\frac{\beta}{\pi} \int_{0}^{\infty} d t \rho^{\prime}(\sqrt{2 J \beta} \cosh t) .
$$




\section{Appendix C}

\section{Index of Symbols}

\begin{tabular}{lll} 
Symbol & Description & Page defined \\
\hline$\alpha$ & Parameter of 1-D linear map & 111 \\
$\alpha$ & Imaginary part of branch in cavity tail impedance & 55 \\
$\alpha_{x}$ & Horizontal $\alpha$ & 24 \\
$\beta$ & Parameter of 1-D linear map & 111 \\
$\beta_{x}$ & Horizontal $\beta$ & 24 \\
$\beta_{0}$ & Velocity divided by c & 13 \\
$\left\langle\beta_{y}\right\rangle$ & Average $\beta$-function & 50 \\
$\left\langle\beta_{y}\right\rangle_{\text {arc }}$ & Average $\beta$-function in arcs & 50 \\
$\left\langle\beta_{y}\right\rangle_{\text {bpm }}$ & Average $\beta$-function at BPM's & 50 \\
$\left\langle\beta_{y}\right\rangle_{\text {cav }}$ & Average $\beta$-function at cavities & 50 \\
$\left\langle\beta_{y}\right\rangle_{\text {str }}$ & Average $\beta$-function in straights & 50 \\
$\Delta$ & Argument in Fourier transforms of distribution & 38 \\
$\delta$ & Dirac delta function & 14 \\
$\delta_{L}$ & Dirac delta function with period $L$ & 21 \\
$\epsilon_{q}$ & Beam emittance & 111 \\
$\epsilon_{x}$ & Horizontal beam emittance & 24 \\
$\epsilon_{y}$ & Vertical beam emittance & 50 \\
$\eta$ & Dispersion function & 22 \\
$\eta C$ & Momentum compaction factor & 37 \\
$\Phi$ & Electromagnetic scalar potential & 17
\end{tabular}

\begin{tabular}{|c|c|c|}
\hline Symbol & Description & Page defined \\
\hline$\phi$ & Transverse cylindrical coordinate & 12 \\
\hline$\phi_{\mathrm{rf}}$ & Phase offset of $\mathrm{rf}$ voltage & 21 \\
\hline$\gamma_{0}$ & Ratio of particle energy to rest mass & 12 \\
\hline$\Gamma^{ \pm}$ & Integration contours & .119 \\
\hline$\lambda_{m}$ & Line density of $m$-th moment of charge distribution & 15 \\
\hline$\mu$ & Phase advance in 1-d system & 110 \\
\hline$\mu^{\prime}$ & Phase advance with feedback & 88 \\
\hline$\Delta \mu$ & Phase advance from pickup to kicker & 81 \\
\hline$\rho$ & Charge density & 11 \\
\hline$\rho$ & Fourier transform of distribution & 38 \\
\hline$\rho_{m}$ & Fourier coefficient of $\rho$ & 14 \\
\hline$\Psi$ & Phase space distribution function & 28 \\
\hline$\Psi_{n}$ & Perturbation to $\Psi_{n 0}$ & 25 \\
\hline$\Psi_{n 0}$ & Time-independent $\hat{\Psi}_{n}$ that solves Vlasov equation & 25 \\
\hline$\hat{\Psi}_{n}$ & Phase space distribution of particles in bucket $n$ & 25 \\
\hline$\psi$ & Phase in solution of 1-d system & 110 \\
\hline$\Sigma_{\alpha}$ & R.m.s. emittance in Gaussian bunch & 42 \\
\hline$\sigma_{r}$ & Transverse bunch size & 13 \\
\hline$\sigma_{\ell}$ & Bunch half-length & 24 \\
\hline$\sigma_{\tau}$ & Bunch half-length & 43 \\
\hline$\sigma$ & Vacuum chamber wall conductivity & 54 \\
\hline$\tau$ & Arrival time offset from synchronous particle & 3 \\
\hline$\tau_{d}$ & Damping time & 31 \\
\hline$\tau_{y}$ & Vertical damping time & 50 \\
\hline$\hat{\tau}$ & Coordinate transform from $\theta, J$ to $\tau$ & 25 \\
\hline$\hat{\tau}_{n 0}$ & Center of the $n$th rf bucket & 21 \\
\hline$\tau_{n 0}$ & Value of $\tau$ in $n$th bucket when $J=0$ & 25 \\
\hline$\theta_{q}$ & Angle variable conjugate to $J_{q}$ & 110 \\
\hline$\theta_{s}$ & Angle variable conjugate to $J_{s}$ & 110 \\
\hline$\theta$ & Vector of angles & 24 \\
\hline $\boldsymbol{\omega}$ & Single particle oscillation frequencies & 5 \\
\hline$\omega_{0}$ & Angular revolution frequency around ring & 31 \\
\hline
\end{tabular}




\begin{tabular}{lll} 
Symbol & Description & Page defined \\
\hline$\omega_{\xi}$ & Chromatic frequency & 49 \\
$\omega_{\mathrm{rf}}$ & Angular frequency of fundamental rf cavity mode & 21 \\
$\omega_{r}$ & Frequency of linear motion in bunch $r$ & 30 \\
$\omega_{C}$ & Impedance roll-off frequency & 55 \\
$\omega_{R}$ & Resonant frequency for resonator impedance & 55 \\
$\omega_{F B}$ & Half-bandwidth of feedback & 79 \\
$\nu$ & Synchrotron tune & 117 \\
$\Delta \nu$ & Synchrotron tune shift & 117 \\
$\nu_{\tau}$ & Linear synchrotron tune & 117 \\
$\nu_{\alpha}$ & Betatron tune & 44 \\
$\nu_{s}$ & Synchrotron tune & 25 \\
$\xi$ & Derivative of the dispersion function & 22 \\
$\xi$ & Chromaticity & 49 \\
$\xi_{\eta}$ & Relative horizontal-longitudinal coupling at cavities & 23 \\
$A$ & Magnetic vector potential & 17 \\
$\mathcal{A}$ & Lie generator for transformation & 108 \\
$A_{m}$ & Fourier coefficient of solution for momentum kick & 13 \\
$A$ & Matrix for linear transformation & 109 \\
$A$ & Matrix that normalizes linear transform & 115 \\
$\hat{A}_{m}$ & Azimuthal Fourier coefficient of $f$ & 13 \\
$A$ & Amplitude of feedback kick & 81 \\
$A_{s, N}^{(n)}$ & Normal $n$th multipole component of $A_{s}$ & 20 \\
$A_{s, S}^{(n)}$ & Skew $n$th multipole component of $A_{s}$ & 20 \\
$a_{3}, a_{4}$ & Generators for nonlinear normalization & 116 \\
$a_{k}$ & Coefficient of $y^{n}$ in expansion of $A_{s}$ & 19 \\
$a^{(n)}$ & Coefficient of $y^{n}$ in expansion of $A_{s}$ & 19 \\
$B$ & Magnetic field & 11 \\
$B_{0}$ & Magnetic bending fields & 20 \\
$B_{q r a}^{\beta \alpha}$ & Term in sum for $R_{q p r}^{\beta \alpha}$ & 33 \\
$b$ & Derivative of $p_{s}$ transformation & 109 \\
$b_{0 k n d}$ & Term in sum of products expansion for $g_{0 k i n}$ & 39 \\
$b_{0 n d}$ & Function whose Taylor coefficients are $b_{0 k n d}$ & 40 \\
& &
\end{tabular}

\begin{tabular}{|c|c|c|}
\hline Symbol & Description & Page defined \\
\hline$b_{\alpha k n m d}$ & Term in sum of products expansion for $f_{a k l n}+\cdots$ & 39 \\
\hline$b_{\text {anmd }}$ & Function whose Taylor coefficients are $b_{\alpha k n m d}$ & 40 \\
\hline$b_{p}$ & Vacuum chamber half-height & 54 \\
\hline C & Curve which is the ideal particle orbit & 3 \\
\hline$C_{0}$ & Constant of integration & 110 \\
\hline$C_{1}$ & Constant of integration & 110 \\
\hline$C_{2}$ & Constant of integration & 110 \\
\hline$C_{p r a}^{\beta \alpha}$ & Term in sum for $R_{q p r}^{\beta \alpha}$ & 33 \\
\hline c & The speed of light & 11 \\
\hline$c_{0 k n d}$ & Term in sum of products expansion for $g_{0 k l n}$ & 39 \\
\hline$c_{0 \text { nd }}$ & Function whose Taylor coefficients are coknid & 40 \\
\hline$c_{\alpha k n m d}$ & Term in sum of products expansion for $f_{a k l n}+\cdots$ & 39 \\
\hline$c_{\text {anmd }}$ & Function whose Taylor coefficients are $c_{\alpha k n m d}$ & 40 \\
\hline$D$ & $Z_{22}-Z_{11}$ & 59 \\
\hline$D_{n p}^{\alpha}$ & Fourier transformed moment of $\Psi_{n}$ & 32 \\
\hline$D_{r a}^{\beta a}$ & Basis function & 33 \\
\hline$\tilde{D}_{\mathrm{ra}}^{\beta \alpha}$ & Basis function for bunch & 36 \\
\hline$\hat{D}_{q_{0} a}^{\beta \alpha}$ & Basis function for multibunch mode & 36 \\
\hline$d_{\alpha}$ & Fourier transform of distribution-related function & 38 \\
\hline$E$ & Beam energy & 50 \\
\hline$\Delta E$ & Energy loss per turn & 114 \\
\hline $\boldsymbol{E}$ & Electric field & 11 \\
\hline$e$ & The charge of a particle & 11 \\
\hline$F$ & Mixed variable generating function & 110 \\
\hline$F$ & Form factor for resistive wall impedance & 54 \\
\hline $\boldsymbol{F}$ & Force on a test particle & 11 \\
\hline$F_{\|}$ & Force parallel to $v$ & 12 \\
\hline$F_{\perp}$ & Force perpendicular to $v$ & 12 \\
\hline$f$ & Scalar function & 12 \\
\hline$f_{3}, f_{4}$ & Nonlinear terms in map & 116 \\
\hline$f$ & Function that phase space is shifted by & 108 \\
\hline$f_{n}^{\alpha}$ & Generalization of $x_{n}$ & 32 \\
\hline
\end{tabular}




\begin{tabular}{|c|c|c|}
\hline Symbol & Description & Page defined \\
\hline$f_{n 0}^{\alpha}$ & Generalization of $x_{n 0}$ & 32 \\
\hline$f_{\alpha}$ & Distribution to be expanded & 38 \\
\hline$f_{\lambda k l n}$ & Expansion coefficient for $f_{\lambda}$ & 39 \\
\hline$f_{\mathrm{xf}}$ & R.F. frequency & 50 \\
\hline$G$ & Operator in Vlasov equation & 33 \\
\hline$H$ & Time-dependent Hamiltonian & 17 \\
\hline$H^{\prime}$ & Hamiltonian in 8 phase space variables & 108 \\
\hline$H_{0}$ & Hamiltonian without wakefield & 24 \\
\hline$H_{r}$ & Hamiltonian for bunch $r$ & 25 \\
\hline$\Delta H_{r}$ & $H_{r}-H_{r 0}$ & 30 \\
\hline$H_{r 0}$ & Hamiltonian for bunch $r$ with potential-well distortion & 26 \\
\hline$h$ & Reciprocal of the local radius of curvature & 17 \\
\hline$h_{4}$ & Nonlinear map remainder after normalization & 116 \\
\hline$h_{\mathrm{rf}}$ & Harmonic number of rf cavity & 21 \\
\hline$I$ & Total beam current & 50 \\
\hline$I_{m}$ & Modified Bessel function & 13 \\
\hline$I_{n}$ & $s$ interval in which each bunch resides & 29 \\
\hline$I_{r}$ & Current in bunch $r$ & 44 \\
\hline$J$ & The symplectic matrix & 108 \\
\hline$J_{\eta}$ & Characteristic action of dispersion & 24 \\
\hline$J_{q}$ & Action variable corresponding to $q$ & 110 \\
\hline$J_{s}$ & Action variable corresponding to $s$ & 110 \\
\hline$J_{0}$ & Zeroth order Bessel function & 118 \\
\hline $\boldsymbol{J}$ & Current density & 11 \\
\hline $\boldsymbol{J}$ & Vector of actions & 24 \\
\hline$K$ & Coefficient of quadrupole field & 20 \\
\hline$K_{p}$ & Integral of $k_{p}$ & 112 \\
\hline$\hat{K}_{p}$ & $K_{p}\left(s_{q}+L\right)$ & 112 \\
\hline$k$ & Wave number in $z$ direction & 13 \\
\hline$k_{p}$ & Coefficient of $p^{2}$ in 1-d Hamiltonian & 109 \\
\hline$k_{q}$ & Coefficient of $q^{2}$ in 1-d Hamiltonian & 109 \\
\hline$k_{B}$ & Number of bunches & 50 \\
\hline
\end{tabular}

\begin{tabular}{|c|c|c|}
\hline Symbol & Description & Page defined \\
\hline$L$ & Circumference of the ring & 21 \\
\hline$L$ & Inductance & 55 \\
\hline$L_{\text {arc }}$ & Total length of arc sections & 50 \\
\hline$L_{\text {str }}$ & Total length of straight sections & 50 \\
\hline$L_{p}$ & Vacuum chamber length & 54 \\
\hline$m$ & The mass of a particle & 17 \\
\hline$M$ & Number of symmetric bunches & 5 \\
\hline$M$ & Longitudinal full-turn map & 115 \\
\hline$M_{r a}^{\beta \alpha}$ & Basis function & 34 \\
\hline$N_{r}$ & Number of particles in bunch $r$ & 25 \\
\hline$N_{\text {cav }}$ & Number of cavities & 50 \\
\hline$P, P_{1}$ & Points for describing storage ring coordinate system & 3 \\
\hline$P$ & $Z_{21} Z_{12}$ & 59 \\
\hline$p$ & Arbitrary momentum for 1-d Hamiltonian & 109 \\
\hline$p_{0}$ & Reference momentum & 18 \\
\hline$p$ & Canonical momentum vector & 17 \\
\hline$p_{s}^{\prime}$ & Negative of the transformed s-dependent Hamiltonian & 108 \\
\hline$\Delta p$ & Momentum gained by test particle & 12 \\
\hline$\Delta p_{\perp}$ & $\Delta p$ perpendicular to $v$ & 12 \\
\hline$\Delta p_{\|}$ & $\Delta p$ parallel to $v$ & 12 \\
\hline$Q$ & Parameter in resonator impedance & 55 \\
\hline$q$ & The charge of a particle & 13 \\
\hline$q$ & Arbitrary coordinate for $1-\mathrm{d}$ Hamiltonian & 109 \\
\hline$R$ & Normalized linear part of map & 116 \\
\hline$R_{q p r}^{\beta \alpha}$ & Matrix element & 33 \\
\hline$R_{\mathrm{FB}}$ & Coefficient for feedback impedance & 79 \\
\hline$R_{\mathrm{Ind}}$ & Coefficient for inductive impedance & 55 \\
\hline$R_{\text {res }}$ & Coefficient for resonator impedance & 55 \\
\hline$R_{\mathrm{RW}}$ & Coefficient for resistive wall impedance & 54 \\
\hline$R_{\text {Tail }}$ & Coefficient for cavity tail impedance & 55 \\
\hline$r$ & Transverse cylindrical coordinate & 12 \\
\hline$S$ & Coefficient of sextupole field & 20 \\
\hline
\end{tabular}




\begin{tabular}{|c|c|c|}
\hline Symbol & Description & Page defined \\
\hline$s$ & Longitudinal position along the curve $C$ & 3 \\
\hline$s_{q}$ & Location of $\delta$-function kick & 111 \\
\hline$s_{\mathrm{zf}}$ & $s$ for rf cavity & 21 \\
\hline$\Delta s$ & Offset of feedback kicker from picker & 26 \\
\hline$t$ & Time & 11 \\
\hline$t_{\mathrm{ref}}$ & Arrival time of reference particle & 15 \\
\hline$T$ & $Z_{22}+Z_{11}$ & 59 \\
\hline$T_{0}$ & Revolution period & 25 \\
\hline$V$ & External potential & 17 \\
\hline$\hat{V}$ & Maximum rf voltage & 21 \\
\hline$v$ & Velocity of a test particle & 11 \\
\hline$W_{m}$ & Wake function & 16 \\
\hline$W_{\perp}$ & $W_{1}$ & 16 \\
\hline$W_{0}^{F B}$ & Longitudinal wake associated with feedback & 27 \\
\hline$W_{x}^{\mathrm{FB}}$ & Horizontal wake associated with feedback & 27 \\
\hline$W_{y}^{\mathrm{FB}}$ & Vertical wake associated with feedback & 27 \\
\hline$w$ & Amplitude in solution of 1-d system & 110 \\
\hline $\boldsymbol{X}$ & Trajectory of test particle & 11 \\
\hline$x$ & Transverse coordinate in the plane of $C$ & 3 \\
\hline$\hat{x}$ & Coordinate transform from $\theta, J$ to $x$ & 25 \\
\hline$x_{n 0}$ & Value of $x$ in $n$th bucket when $J=0$ & 25 \\
\hline$x$ & Canonical coordinate vector & 17 \\
\hline$x_{0}$ & Initial condition for trajectory of test particle & 11 \\
\hline$y$ & Transverse coordinate perpendicular to the plane of $C$ & 3 \\
\hline$\hat{y}$ & Coordinate transform from $\theta, J$ to $y$ & 25 \\
\hline$y_{n 0}$ & Value of $y$ in $n$th bucket when $J=0$ & 25 \\
\hline$Z_{0}$ & Impedance of free space & 54 \\
\hline$Z_{i j}$ & Matrix elements for eigenvalue system & 57 \\
\hline$Z_{m}^{\frac{1}{m}}$ & Transverse impedance & 17 \\
\hline$Z_{m}^{\|}$ & Longitudinal impedance & 17 \\
\hline$Z_{x}^{F B}$ & Horizontal feedback impedance & 28 \\
\hline$Z_{y}^{F B}$ & Vertical feedback impedance & 28 \\
\hline
\end{tabular}

\begin{tabular}{|c|c|c|}
\hline Symbol & Description & Page defined \\
\hline$Z_{\perp}$ & $Z_{1}^{1}$ & 17 \\
\hline$Z_{\perp}^{\text {Ind }}$ & Inductive transverse impedance & 55 \\
\hline$Z_{\perp}^{\text {res }}$ & Resonator transverse impedance & 55 \\
\hline$Z_{\perp}^{\mathrm{RW}}$ & Resistive wall transverse impedance & 54 \\
\hline$z_{\|}^{\text {Thail }}$ & Cavity tail longitudinal impedance & 55 \\
\hline$z_{\|}^{\prime \prime}$ & $z_{0}^{\|}$ & 17 \\
\hline$Z_{\|}^{\mathrm{FB}}$ & Longitudinal feedback impedance & 28 \\
\hline$Z_{\alpha}$ & Generalized impedance & 32 \\
\hline$Z_{\alpha}^{F B}$ & Generalized feedback impedance & 45 \\
\hline$Z_{q k b a n r}^{\gamma \beta \alpha}$ & Impedance integrated over $s$ times $r$ pieces & 35 \\
\hline$\tilde{Z}_{q_{0} k b a n r}^{\gamma \beta \alpha}$ & Impedance associated with multibunch mode $q_{0}$ & 36 \\
\hline$\hat{Z}_{q 0 k b a}^{\gamma \beta a}$ & $\tilde{Z}_{q_{0} k b a n r}^{\gamma \beta \alpha}$ when bunches have same distribution & 36 \\
\hline$z$ & Cylindrical coordinate parallel to $v$ & 12 \\
\hline$z$ & Phase space coordinate vector & 108 \\
\hline$z_{\text {ref }}$ & Initial condition for reference particle & 15 \\
\hline$\Re$ & Real part & \\
\hline$\Im$ & Imaginary part & \\
\hline$z^{*}$ & Complex conjugate of $z$ & \\
\hline$z^{T}$ & Transpose of the vector $z$ & \\
\hline\langle\rangle & Average over the beam distribution & \\
\hline [] & Poisson brackets & 28 \\
\hline$\lceil z\rceil$ & Smallest integer $\geq z$ & \\
\hline
\end{tabular}


[9] D. Brandt and B. Zotter. Simulation of transverse stability of single bunches in storage rings. In Cole and Donaldson [17], pages 309-311.

[10] Yunhai Cai. Private communication.

\section{Bibliography}

[1] Milton Abramowitz and Irene A. Stegun, editors. Handbook of Mathematical Functions. Dover, New York, 1972.

[2] A. I. Akhiezer, I. A. Akhiezer, R. V. Polovin, A. G. Sitenko, and K. N. Stepanov. Plasma Electrodynamics. Pergamon, Oxford, 1975.

[3].V. I. Arnold. Mathematical Methods of Classical Mechanics. Springer-Verlag, New York, second edition, 1989.

[4] ASM International Handbook Committee, Materials Park, Ohio. Metals Handbook, tenth edition, 1990.

[5] J. Scott Berg. Observations involving broadband impedance modelling. Technical Report SLAC-PUB-95-6964, Stanford Linear Accelerator Center, Stanford, California, August 1995. To appear in the proceedings of the International Workshop on Collective Effects and Impedance for B-Factories, Tsukuba, Japan, 12-17 June, 1995.

[6] J. Scott Berg and Ronald D. Ruth. Transverse instabilities for multiple nonrigid bunches in a storage ring. Phys. Rev. E, 52(3):R2179-2182, September 1995.

[7] J. Scott Berg and Ronald D. Ruth. Transverse multibunch instabilities for non-rigid bunches. Technical Report SLAC-PUB-95-6830, Stanford Linear Accelerator Center, Stanford, CA, April 1995. Presented at the 16th IEEE Particle Accelerator Conference (PAC95) and International Conference on High-Energy Accelerators, Dallas, Texas, May 1-5, 1995.

[8] J. Scott Berg and Ronald D. Ruth. Transverse multibunch modes for non-rigid bunches, including mode coupling. Technical Report SLAC-PUB-95-6965, SLAC, Stanford, CA, August 1995.
[11] Alexander Wu Chao. Physics of Collective Beam Instabilities in High Energy Accelerators. John Wiley \& Sons, Inc., New York, 1993.

[12] Y. H. Chin. Coherent synchro-betatron resonances driven by localized wake fields. Technical Report CERN-SPS/85-33, CERN, Geneva, Switzerland, July 1985.

[13] Y. H. Chin. User's guide for $A B C I$ version 8.8 (azimuthal beam cavity interaction). Technical Report LBL-35258, Lawrence Berkeley Laboratory, Berkeley, CA, February 1994.

[14] Y. H. Chin and K. Yokoya. Landau damping of a multi-bunch instability due to bunchto-bunch tune spread. Technical Report DESY 86-097, DESY, Hamburg, Germany, August 1986.

[15] Yong Ho Chin. Hamiltonian formulation for transverse bunched beam instabilities in the presence of betatron tune spread. Technical Report CERN SPS/85-9, CERN, Geneva, Switzerland, May 1985.

[16] Yongho Chin, Kohtaro Satoh, and Kaoru Yokoya. Instability of a bunched beam with synchrotron-frequency spread. Part. Accel., 13:45-66, 1983.

[17] Francis T. Cole and Rene Donaldson, editors. Proceedings of the 12th International Conference on High Energy Accelerators, Batavia, II, August 1983. Fermi National Accelerator Laboratory.

[18] M. Cornacchia and J.M. Wang. Theoretical treatment of transverse feedback systems with memory. IEEE Trans. Nucl. Sci., 28:2255-2257, 1981.

[19] E. D. Courant, R. D. Ruth, and W. T. Weng. Stability in dynamical systems I. In Melvin Month, Per F. Dahl, and Margaret Dienes, editors, Physics of High Energy Accelerators (BNL/SUNY Summer School, 1983), number 127 in AIP Conference Proceedings, pages 294-343, New York, 1983. American Institute of Physics. 
[20] Ronald C. Davidson. Methods in Nonlinear Plasma Theory. Academic Press, New York, 1972.

[21] Alex J. Dragt. Lectures on nonlinear orbit dynamics. In R. A. Carrigan, F. R. Huson, and M. Month, editors, Physics of High Energy Particle Accelerators (Fermilab Summer School, 1981), number 87 in AIP Conference Proceedings, pages 147-313, New York, 1982. American Institute of Physics.

[22] Alex J. Dragt and John M. Finn. Lie series and invariant functions for analytic symplectic maps. J. Math. Phys., 17(12):2215-2227, December 1976.

[23] Alex J. Dragt and John M. Finn. Normal form for mirror machine hamiltonians. $J$. Math. Phys., 20(12):2649-2660, December 1979.

[24] Alex J. Dragt and Etienne Forest. Computation of nonlinear behavior of hamiltonian systems using Lie algebraic methods. J. Math. Phys., 24(12):2734-2744, December 1983.

[25] Alex J. Dragt, Filippo Neri, Govindan Rangarajan, David R. Douglas, Liam M. Healy, and Robert D. Ryne. Lie algebraic treatment of linear and nonlinear beam dynamics. Ann. Rev. Nucl. Part. Sci., 38:455-496, 1988.

[26] Etienne Forest, Martin Berz, and John Irwin. Normal form methods for complicated periodic systems: A complete solution using differential algebra and Lie operators. Part. Accel., 24:91-107, 1989.

[27] Étienne Forest, Leo Michelotti, Alex J. Dragt, and J. Scott Berg. The modern approach to single-particle dynamics for circular rings. In Melvin Month, Alessandro G. Ruggiero, and Wu-Tsung Weng, editors, Stability of Particle Motion in Storage Rings, Upton, NY 1992, number 292 in AIP Conference Proceedings, pages 417-487, New York, 1994. American Institute of Physics.

[28] Robert L. Gluckstern, Johannes van Zeijts, and Bruno Zotter. Coupling impedance of beam pipes of general cross section. Phys. Rev. E, 47(1):656-663, January 1993.

[29] Gene H. Golub and Charles F. Van Loan. Matrix Computations. Johns Hopkins University Press, Baltimore, second edition, 1989.
[30] J. Haïssinski. Exact longitudinal equilibrium distribution of stored electrons in the presence of self-fields. Il Nuovo Cimento, 18B(1):72-82, 1973.

[31] S. Heifets, K. Ko, X. Lin, A. Chao, G. Stupakov, M. Zolotorev, J. Seeman, U. Weinands, C. Perkins, M. Nordby, E. Daly, N. Kurita, D. Wright, E. Henestroza, G. Lambertson, J. Corlett, J. Byrd, M. Zisman, T. Weiland, W. Stoeff, and C. Belser. Impedance study for the PEP-II $B$-factory. Technical Report SLAC/AP-99, Stanford Linear Accelerator Center, Stanford, CA, March 1995.

[32] S. A. Heifets and S. A. Kheifets. High-frequency limit of the longitudinal impedance of an array of cavities. Phys. Rev. D, 39(3):960-970, February 1989.

[33] Sam Heifets. Private communication.

[34] H. G. Hereward. Landau damping by non-linearity. Technical Report CERN/MPS/DL 69-11, CERN, Geneva, Switzerland, July 1969.

[35] Don Hunt. Private communication.

[36] S. Ichimaru. Basic Principles of Plasma Physics. W. A. Benjamin, Reading, Mass., 1973.

[37] J. S. Jackson. Classical Electrodynamics. John Wiley \& Sons, New York, second edition, 1975.

[38] R. D. Kohaupt. Head tail turbulence and the transverse petra instability. Technical Report DESY 80/22, DESY, Hamburg, Germany, March 1980.

[39] R. D. Kohaupt. On multi-bunch instabilities for fractionally filled rings. Technical Report DESY-85-139, DESY, Hamburg, Germany, December 1985.

[40] J. L. Laclare. Bunched-beam instabilities. In Newman [45], pages 526-538.

[41] A. J. Lichtenberg and M. A. Lieberman. Regular and Chootic Dynamics, volume 38 of Applied Mathematical Sciences. Springer-Verlag, New York, second edition, 1992

[42] S. R. Mane. Solutions of laplace's equation in two dimensions with a curved longitudinal axis. Nucl. Instrum. and Methods, A321:365-375, 1992 
[43] Leo Michelotti. Intermediate Classical Dynamics with Applications to Beam Physics. John Wiley \& Sons, Inc., New York, 1995.

[44] S. Myers. Effect of reactive feedback on the transverse mode coupling instability using the few-particles model and simulation. IEEE Trans. Nucl. Sci., NS-32(5):2341-2343, October 1985 .

[45] W. S. Newman, editor. 11th International Conference on High-Energy Accelerators, Basel, July 1980. European Organization for Nuclear Research (CERN), Birkhäuser Verlag.

[46] Katsunobu Oide. Effects of the potential-well distortion on the longitudinal singlebunch instability. In K. Hirata and T. Suzuki, editors, Fourth Advanced ICFA Beam Dynamics Workshop on Collective Effects in Short Bunches, pages 64-72, Tsukuba, Japan, February 1991. KEK. KEK Report 90-21.

[47] Katsunobu Oide. A mechanism of longitudinal single-bunch instability in storage rings. Part. Accel., 51:43-52, 1995.

[48] Katsunobu Oide and Kaoru Yokoya. Longitudimal single-bunch instability in electron storage rings. Technical Report KEK Preprint 90-10, KEK, Tsukuba, Japan, April 1990.

[49] PEP-II an asymmetric $B$ factory, conceptual design report. Technical Report SLAC418, LBL-PUB-5379, CAIT-68-1869, UCRL-ID-114055, UC-IIRPA-93-01, 1993.

[50] PEP-II machine advisory committee meeting, August 24-25, 1995.

[51] PEP-II machine advisory committee meeting, January 4-6, 1995.

[52] E. Peschardt. Compensation of the resistive and reactive transverse wall impedance in the ISR with a feedback system. In Newman [45], pages 506-510.

[53] Robert Rimmer. Private communication.

[54] Walter Rudin. Real and Complex Analysis. McGraw-Hill, New York, third edition, 1987.

[55] F. Ruggiero. Transverse mode coupling instability due to localized structures. Part. Accel., 20:45-64, 1986
[56] R. Ruth. Reactive feedback in the two particle model. Technical Report CERN-LEPTH/83-22, CERN, Geneva, Switzerland, 1983.

[57] R. D. Ruth. Vertical Coherent Instabilities in Bunched Particle Beams. PhD thesis, State University of New York at Stony Brook, Upton, NY, July 1981. Brookhaven National Laboratory report BNL 51425.

[58] Ronald D. Ruth. Transverse mode coupling with feedback. In Cole and Donaldson [17], pages 389-392.

[59] F. J. Sacherer. Transverse bunched beam instabilities-theory. In Proceedings of the IXth International Conference on High Energy Accelerators, pages 347-351, Stanford, California, May" 1974. International Union of Pure and Applied Physics, National Science Foundation, and U.S. Atomic Energy Commission. Report numbers CONF 740522, UC-28-Accelerators.

[60] F. J. Sacherer. Bunch lengthening and microwave instability. IEEE Trans. Nucl. Sci., NS-24(3):1393-1395, June 1977.

[61] J. J. Sakurai. Modern Quantum Mechanics. Addison-Wesley, Redwood City, California, 1985.

[62] Matthew Sands. The physics of electron storage rings; an introduction. Technical Report SLAC-121, UC-28, SLAC and the University of California, Santa Cruz, 1970.

[63] Kohtaro Satoh and Yongho Chin. Transverse mode coupling in a bunched beam. Nucl. Instrum. and Methods, 207:309-320, 1983.

[64] Toshio Suzuki. Transverse mode coupling instability due to piecewise constant impedances. Technical Report CERN-LEP-TH/87-55, CERN, Geneva, Switzerland, August 1987.

65] Richard Talman. The influence of finite synchrotron oscillation frequency on the transverse head-tail effect. Nucl. Instrum. and Methods, 193:423-435, 1982.

[66] K. A. Thompson and R. D. Ruth. Transverse couplied-bunch instabilities in damping rings of high-energy linear coiliders. Phys. Rev. D, 43(9):3049-3062, May 1991. 


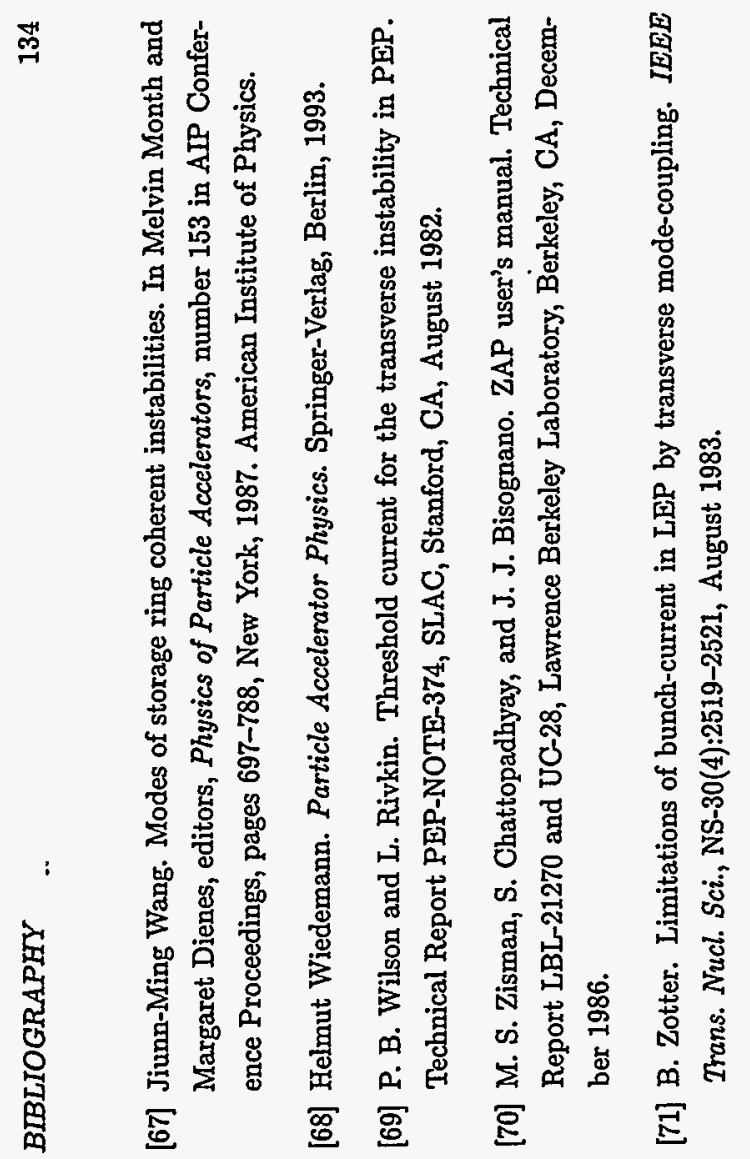




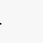

